\title{
THE GEOMETRY OF FROBENIOIDS I: THE GENERAL THEORY
}

\author{
Shinichi MOCHIZUKI \\ (Received 2 May 2006 and revised 17 January 2008)
}

\begin{abstract}
We develop the theory of Frobenioids, which may be regarded as a categorytheoretic abstraction of the theory of divisors and line bundles on models of finite separable extensions of a given function field or number field. This sort of abstraction is analogous to the role of Galois categories in Galois theory or monoids in the geometry of log schemes. This abstract category-theoretic framework preserves many of the important features of the classical theory of divisors and line bundles on models of finite separable extensions of a function field or number field such as the global degree of an arithmetic line bundle over a number field, but also exhibits interesting new phenomena, such as a 'Frobenius endomorphism' of the Frobenioid associated to a number field.
\end{abstract}

\section{Contents}

$\begin{array}{lr}\text { Introduction } & \mathbf{2 9 4}\end{array}$

I1 Technical summary . . . . . . . . . . . . . . . . . . . . . . . . . . . . . . . 294

I2 Abstract combinatorialization of arithmetic geometry . . . . . . . . . . . 296

I3 Frobenius endomorphisms of a number field . . . . . . . . . . . . . . 298

I4 Étale-like versus Frobenius-like structures . . . . . . . . . . . . . . . . . 300

0 Notation and conventions $\quad \mathbf{3 0 0}$

1 Definitions and first properties $\quad 308$

2 Frobenius functors $\quad \mathbf{3 3 0}$

3 Category-theoreticity of the base and Frobenius degree 340

4 Category-theoreticity of the divisor monoid 357

5 Model Frobenioids $\quad 376$

6 Some motivating examples $\quad 388$

Appendix. Slim exponentiation $\quad 395$

Chart of types of morphisms in a Frobenioid $\quad 396$

References $\quad 396$

$\begin{array}{ll}\text { Index } & 397\end{array}$

2000 Mathematics Subject Classification: Primary 14A99; Secondary 11G99.

Keywords: categories; Galois categories; Frobenius; monoids; log schemes.

(c) 2008 Faculty of Mathematics, Kyushu University 


\section{Introduction}

\section{I1. Technical summary}

In this paper, we introduce the notion of a Frobenioid. The simplest kind of Frobenioid ' $\mathbb{F}_{M}$ ' is the non-commutative monoid given by forming the 'semi-direct product monoid' of a given commutative monoid $M$ with the multiplicative monoid of positive integers $\mathbb{N}_{\geq 1}$ (cf. Section 0 ), where $n \in \mathbb{N}_{\geq 1}$ acts on $M$ by multiplication by $n$; that is to say, the underlying set of $\mathbb{F}_{M}$ is the product

$$
M \times \mathbb{N}_{\geq 1}
$$

equipped with the monoid structure given as follows: if $a_{1}, a_{2} \in M, n_{1}, n_{2} \in \mathbb{N}_{\geq 1}$, then $\left(a_{1}, n_{1}\right) \cdot\left(a_{2}, n_{2}\right)=\left(a_{1}+n_{1} \cdot a_{2}, n_{1} \cdot n_{2}\right)$ (cf. Definition 1.1(iii)). For instance, when $M$ is taken to be the additive monoid of non-negative integers $\mathbb{Z}_{\geq 0}$ (cf. Section 0 ), we shall write $\mathbb{F} \stackrel{\text { def }}{=} \mathbb{F}_{\mathbb{Z}_{>0}}$ and refer to $\mathbb{F}$ as the standard Frobenioid. Note that in general, any monoid (such as $\mathbb{F}_{M}$, for instance) may be thought of as a category, i.e., the category with precisely one object whose monoid of endomorphisms is the given monoid.

More generally, one may start with a 'family of commutative monoids' $\Phi$ on a 'base category' $\mathcal{D}$ (where $\Phi$ and $\mathcal{D}$ satisfy certain properties) and form the associated elementary Frobenioid $\mathbb{F}_{\Phi}$ by taking the 'semi-direct product' of $\mathbb{N}_{\geq 1}$ with $\Phi$ (cf. Definition 1.1(iii), for more details). Here, $\mathbb{F}_{\Phi}$ is a category.

In general, a Frobenioid $\mathcal{C}$ is a category equipped with a functor $\mathcal{C} \rightarrow \mathbb{F}_{\Phi}$ to an elementary Frobenioid $\mathbb{F}_{\Phi}$ satisfying certain properties (cf. Definition 1.3 for more details) to the effect that the structure of $\mathcal{C}$ is 'substantially reflected' in this functor $\mathcal{C} \rightarrow \mathbb{F}_{\Phi}$. From the point of view of conventional arithmetic geometry, a Frobenioid may be thought of as a sort of category-theoretic abstraction of the theory of divisors and line bundles on models of finite separable extensions of a given function field or number field. That is to say, the base category $\mathcal{D}$ corresponds to the category of models of finite separable extensions of a given function field or number field; the functor $\Phi$ corresponds to the divisors on such models; the ' $\mathbb{N}_{\geq 1}$ portion' of $\mathbb{F}_{\Phi}$ corresponds to the operation of multiplying a divisor by an element $n \in \mathbb{N}_{\geq 1}$ (or, if one considers the line bundle associated to such a divisor, to the operation of forming the $n$th tensor power of the line bundle).

In some sense, the main result of the theory of this paper is the following.

Under various technical conditions, the functor $\mathcal{C} \rightarrow \mathbb{F}_{\Phi}$ that determines the structure of $\mathcal{C}$ as a Frobenioid may be reconstructed purely categorytheoretically, i.e., from the structure of $\mathcal{C}$ as a category (cf. Corollary 4.11).

These technical conditions are typically satisfied by Frobenioids that arise naturally from arithmetic geometry (cf. Theorems 6.2 and 6.4). Also, we observe that these technical conditions appear unlikely to be superfluous. Indeed, we also give various examples, involving Frobenioids which do not satisfy a variety of these technical conditions, of equivalences of categories with respect to which various portions of the functor $\mathcal{C} \rightarrow \mathbb{F}_{\Phi}$ are not preserved (cf. Examples 3.5, 3.6, 3.7, 3.8, 3.9, 3.10 and 4.3).

Perhaps the most fundamental example of this phenomenon of 'the intrinsic categorytheoretic reconstruction of $\mathcal{C} \rightarrow \mathbb{F}_{\Phi}$ from $\mathcal{C}$ ' is the following. The prototype of a base category 
$\mathcal{D}$ is given by (the subcategory of connected objects of) a Galois category, i.e., a category in which the monoids of endomorphisms of objects have the structure of finite groups. On the other hand, the prototype of the 'non-base category portion' of a Frobenioid, i.e., the 'relative structure of $\mathcal{C}$ over $\mathcal{D}$ ', is given by the monoid ' $\mathbb{F}$ ' (or, more generally, the monoids ' $\mathbb{F}_{M}$ ') discussed above. Then one central aspect of the phenomenon that 'the relative structure of $\mathcal{C}$ over $\mathcal{D}$ is never confused with the structure of $\mathcal{D}$ ' is illustrated by the following easily verified observation:

If $G$ is a finite group, then any homomorphism of monoids $\mathbb{F} \rightarrow G$ factors through the natural surjection $\mathbb{F} \rightarrow \mathbb{N}_{\geq 1}$.

(We refer to Remark 3.1.2 for more details.) Note that this property fails to hold if, for instance, one replaces $\mathbb{F}=\mathbb{F}_{\mathbb{Z}_{\geq 0}}$ by $\mathbb{Z}_{\geq 0}$ (and the surjection $\mathbb{F} \rightarrow \mathbb{N}_{\geq 1}$ by the surjection $\left.\mathbb{Z}_{\geq 0} \rightarrow\{0\}\right)$. Put another way, this property may be thought of as a consequence of the nonabelian nature of $\mathbb{F}$. In particular, if one thinks of the category-theoretic reconstructibility of the functor ' $\mathcal{C} \rightarrow \mathbb{F}_{\Phi}$ ' as a sort of rigidity, then this property is vaguely reminiscent of the 'extraordinary rigidity' asserted by Grothendieck in descriptions of his anabelian philosophy.

After defining and examining the first properties of Frobenioids in Section 1, in Section 2, we discuss various versions of 'Frobenius functors' on Frobenioids, which are intended as category-theoretic abstractions of the Frobenius morphism in positive characteristic algebraic geometry (cf. Remark 6.2.1). In Section 3, we begin the category-theoretic reconstruction of the functor ' $\mathcal{C} \rightarrow \mathbb{F}_{\Phi}$ ' by showing that, under certain conditions, the base category and 'Frobenius degree' (i.e., in effect, the ' $\mathbb{N}_{\geq 1}$ portion of $\mathbb{F}_{\Phi}$ ') may be reconstructed categorytheoretically (cf. Theorem 3.4). In the theory of Section 3, we apply a certain purely categorytheoretic technique, which we shall refer to as 'slim exponentiation'; this technique is entirely independent of the theory of Frobenioids and is discussed in detail in the Appendix. In Section 4 , we then complete the category-theoretic reconstruction of the functor ' $\mathcal{C} \rightarrow \mathbb{F}_{\Phi}$ ' by showing that, under certain conditions, the divisor monoid $\Phi$ may also be reconstructed category-theoretically (cf. Theorem 4.9). In Section 5, we study the extent to which, under certain conditions, one may write down 'explicit models' of fairly general Frobenioids, in a fashion reminiscent of the explicit description of the elementary Frobenioid $\mathbb{F}_{\Phi}$ (cf. Theorem 5.2). This study leads naturally to the investigation of various auxiliary structures on a Frobenioid, namely, base sections and base-Frobenius pairs, that may be used to relate a given Frobenioid satisfying certain conditions to such a 'model Frobenioid' (cf. Theorem 5.2(iv)).

One important technique in the theory of Sections 3-5 is the operation of passing from a Frobenioid to the perfection or realification of the Frobenioid. Roughly speaking, from the point of view of the monoid $\mathbb{F}=\mathbb{F}_{\mathbb{Z}_{>0}}$ introduced above, these operations correspond, respectively, to passing from ' $\mathbb{Z}_{\geq 0}$ ' to the monoids ' $\mathbb{Q}_{\geq 0}$ ' (in the case of the perfection) or ' $\mathbb{R}_{\geq 0}$ ' (in the case of the realification). Another important technique in this theory is the operation of passing to the birationalization of a Frobenioid. This may be thought of as a category-theoretic abstraction of the notion of 'working with rational functions' in algebraic geometry; alternatively, from the point of view of the monoid $\mathbb{F}=\mathbb{F}_{\mathbb{Z}_{>0}}$ introduced above, it may be thought of as corresponding to the operation of passing from ' $\mathbb{Z} \geq 0$ ' to the groupification ' $\mathbb{Z}$ ' of $\mathbb{Z}_{\geq 0}$ (see Table 1 ). 
TABLE 1. Effect on monoids of various basic operations.

\begin{tabular}{ll}
\hline Operation & Effect on $\mathbb{Z}_{\geq 0} \subseteq \mathbb{F}$ \\
\hline Perfection & $\mathbb{Z}_{\geq 0} \rightsquigarrow \mathbb{Q}_{\geq 0}$ \\
Realification & $\mathbb{Z}_{\geq 0} \rightsquigarrow \mathbb{R}_{\geq 0}$ \\
Birationalization & $\mathbb{Z}_{\geq 0} \rightsquigarrow \mathbb{Z}^{\mathbf{2}}$ \\
\hline
\end{tabular}

Finally, in Section 6, we consider the main motivating examples of Frobenioids that arise from number fields and function fields. In particular, we observe in passing that this 'Frobenioid-theoretic formulation of the elementary arithmetic of number fields' also gives rise to some interesting 'Frobenioid-theoretic interpretations' of such classical results in number theory as the Dirichlet unit theorem and Tchebotarev's density theorem, as well as a result in transcendence theory due to Lang (cf. Theorem 6.4(i), (iii) and (iv)).

\section{I2. Abstract combinatorialization of arithmetic geometry}

From a somewhat more conceptual point of view, one central theme of the present paper is the goal of 'abstract combinatorialization of scheme-theoretic arithmetic geometry'. Classical examples of this phenomenon of 'abstract combinatorialization' may be seen in the theory of Galois categories or the theory of monoids in the geometry of log schemes (or, more classically, toric varieties). That is to say, even if one starts by considering various finite étale coverings of schemes, the associated Galois category is a purely 'abstract combinatorial' mathematical object that captures the 'Galois structure' of the various coverings involved in a fashion that is entirely independent of scheme theory. In a similar vein, although a monoid of the sort that appears in log geometry arises as a submonoid of the multiplicative monoid determined by some commutative ring, the 'abstract combinatorial' structure of such a monoid is sufficient to capture various essential properties (such as normality, etc.) of the ring structure of the ambient ring in a fashion that is entirely independent of ring/scheme theory.

A somewhat less classical example of this phenomenon of 'abstract combinatorialization of scheme theory' is given by the theory of [Mzk8], where it is shown that very general locally noetherian log schemes may be 'represented' by categories, in the sense that equivalences between such categories arise from uniquely determined isomorphisms of log schemes. The theory of [Mzk8] is generalized in [Mzk9] so as to take into account the archimedean primes of log schemes which are locally of finite type over a Zariski localization of (the ring of rational integers) $\mathbb{Z}$. As is discussed in the introduction to [Mzk8], this kind of result is motivated partly by the anabelian philosophy of Grothendieck, but perhaps more essentially by the idea that instead of working with set-theoretic objects, such as schemes or log schemes, one should regard categories - which may be thought of as 'abstract combinatorial' mathematical objects constituted by some abstract collection of arrows - as the 'fundamental, primitive objects' of mathematics discourse. Thus, Grothendieck's anabelian philosophy may be regarded as a 'special case' of this point of view, i.e., the case where the categories in 
question are Galois categories

$$
\left(\begin{array}{c}
\text { abstract, } \\
\text { combinatorial } \\
\text { mathematical } \\
\text { objects }
\end{array}\right) \supseteq\left(\begin{array}{c}
\text { categories } \\
- \text { i.e., abstract } \\
\text { collections } \\
\text { of arrows }
\end{array}\right) \supseteq\left(\begin{array}{c}
\text { Galois } \\
\text { categories }
\end{array}\right)
$$

(cf. the 'absolute anabelian geometry' developed in [Mzk5, Mzk6, Mzk7, Mzk10, Mzk11, Mzk12, Mzk14]).

One important drawback of the 'anabelian branch' of this category-theoretic approach to mathematics is that although it is very well suited to capturing essential aspects of the geometry of schemes at non-archimedean primes, it is ill suited to capturing the archimedean aspects of the geometry of schemes, and, in particular, those aspects of the global geometry of schemes over number fields - such as heights - that are of interest in Diophantine geometry. Thus, from this point of view, the extension given in [Mzk9] of the theory of [Mzk8] has the virtue, relative to anabelian geometry, of providing a natural way to incorporate such archimedean and global phenomena as the global degree of an arithmetic line bundle over a number field (cf. [Mzk9, Example 5.1]) into the above-mentioned category-theoretic approach to mathematics.

The approach of [Mzk9], however, has the following fundamental drawback: the categories of [Mzk9] are quite 'large' and 'complicated' by comparison to Galois categories, in the sense that they include a very diverse collection of arithmetic schemes, by comparison to the finite étale coverings of a fixed scheme. This makes it relatively easy to reconstruct the original arithmetic log scheme from the category. On the other hand, this relative ease of reconstruction is a reflection, in essence, of the fact that the geometry of such categories is really not so different from the conventional geometry of arithmetic log schemes. Thus, in other words, one does not gain very much in the way of essentially new geometric phenomena by working with such categories, relative to the conventional geometry of arithmetic log schemes.

By contrast, the relatively simple structure of Galois categories (cf. also the categories of [Mzk13]) makes it much more difficult to reconstruct the scheme from the category indeed, such a reconstruction is only possible in the case of very special 'anabelian' schemes - but, on the other hand, this difficulty of reconstruction may be regarded as a reflection of the fact that there is indeed some interesting new geometry that arises from working with Galois categories that does not exist in the conventional geometry of schemes. Perhaps the most fundamental example of this phenomenon is the well-known fact that the absolute Galois groups of non-isomorphic finite fields are isomorphic. Another less elementary example of this phenomenon is the well-known fact that the Galois category associated to a non-archimedean mixed-characteristic local field (i.e., a finite extension of the $p$-adic number field) admits self-equivalences (i.e., the associated absolute Galois group admits automorphisms) that do not arise from scheme theory (i.e., from an isomorphism of fields - cf., for example, [NSW, p. 674]).

Put another way, the difference between the 'geometry of categories' - i.e., the approach to arithmetic geometry constituted by working with the strictly category-theoretic properties of categories - and the classical approach to arithmetic geometry constituted by working with set-theoretic objects equipped with various complicated auxiliary structures may be regarded 
as analogous to the difference between working with the notion of an abstract group and working with groups of explicit matrices. That is to say, working with strictly group-theoretic properties of abstract groups allows one to contemplate various structures that are common to various distinct groups of explicit matrices, but which are not so evident if one happens to be ignorant of the notion of an 'abstract group' and hence obliged to restrict oneself to manipulations involving explicit matrices.

This state of affairs prompts the following question:

Can one perhaps represent certain special arithmetic log schemes of interest by categories whose 'level of complexity' is closer to Galois categories (i.e., substantially simpler than the categories of [Mzk9]) - thus allowing one to hope that the geometry of such categories exhibits fundamentally new phenomena that do not appear in the conventional geometry of arithmetic log schemes - on the one hand, but which nevertheless allow one to work naturally with archimedean primes and heights on the other?

This sort of question constituted one of the principal motivations for the author to develop the theory discussed in this paper.

The answer to the above question constituted by the theory of this paper is, in a word, the notion of a Frobenioid. From the point of view of the question posed above:

Frobenioids provide a single framework (cf. the notion of a 'Galois category'; the role of monoids in log geometry) that allows one to capture the essential aspects of both the Galois and the divisor theory of number fields, on the one hand, and function fields, on the other, in such a way that one may continue to work with, for instance, global degrees of arithmetic line bundles on a number field, but which also exhibits the new phenomenon (not present in the classical theory of number fields) of a 'Frobenius endomorphism' of the Frobenioid associated to a number field.

Here, we remark that the base category $\mathcal{D}$ is typically a category that is of a level of 'simplicity' (cf. the above discussion) that is reminiscent of a Galois category (cf. also the 'temperoids' of [Mzk11]; the categories of Riemann surfaces discussed in [Mzk13, Section 2]). Indeed, in the examples of Section 6, the base category is (the subcategory of connected objects of) a Galois category. From this point of view, the main ingredients of a Frobenioid - that is to say, roughly speaking, 'Galois' (i.e., the base category $\mathcal{D}$ ), 'Frobenius' (i.e., ' $\mathbb{N}_{\geq 1}$ ') and 'metrics/integral structures' (i.e., the family of monoids $\Phi$ ) - are reminiscent of the theory of the 'ring of p-adic periods' $\mathbb{B}_{\text {crys }}$ of $p$-adic Hodge theory.

\section{I3. Frobenius endomorphisms of a number field}

From a somewhat less conceptual point of view, one of the main motivations for the author in developing the theory of Frobenioids came from the long-term goal of developing a sort of arithmetic Teichmüller theory for number fields equipped with an elliptic curve, in a fashion that is analogous to the $p$-adic Teichmüller theory of [Mzk1, Mzk2]. That is to say, here one wishes to regard number fields as corresponding to hyperbolic curves over finite fields and 
elliptic curves (over a number field) as corresponding to the nilpotent ordinary indigenous bundles (on a hyperbolic curve over a finite field) of [Mzk1, Mzk2].

In the $p$-adic Teichmüller theory of [Mzk1, Mzk2], certain canonical Frobenius liftings play a central role. Thus, since Frobenius liftings are, literally, liftings of the Frobenius morphism in positive characteristic, in order to develop an 'arithmetic Teichmüller theory' for number fields equipped with an elliptic curve, one must first have an analogue for number fields of the Frobenius morphism in positive characteristic scheme theory. If one starts to consider such an analogue from a completely naive point of view, then one must contend with the fact that, if, for instance, $n \geq 2$ is a integer, then the morphism

$$
p \mapsto p^{n}
$$

(where $p$ is a prime number) clearly does not extend to a ring homomorphism $\mathbb{Z} \rightarrow \mathbb{Z}$ ! That is to say, it is difficult to see how to accommodate such a 'Frobenius morphism for number fields' within the framework of scheme theory.

On the other hand, if one works with monoids as in the theory of log schemes, then such a morphism ' $p \mapsto p^{n}$, does indeed make sense. Moreover, even if, for instance, one considers roots $\pi$ of $p$, the mapping $\pi \mapsto \pi^{n}$ is Galois-equivariant. Thus, in summary:

One important motivation for the author in developing the theory of Frobenioids was the goal of developing a geometric framework - i.e., roughly speaking, a geometry built up solely from 'Galois theory' and 'monoids' - in which a 'Frobenius morphism on number fields' may be constructed.

Once one has constructed such a 'Frobenius morphism on number fields', the next step to realizing an 'arithmetic Teichmüller theory' consists of constructing a 'canonical Frobenius lifting'. Although the construction of such 'canonical Frobenius liftings' lies (well!) beyond the scope of this paper, we remark that the ideas that lie behind such a construction are motivated by the (scheme-theoretic!) Hodge-Arakelov theory of elliptic curves surveyed in [Mzk3, Mzk4], a theory in which the theta function on a Tate curve plays a central role. In particular, in order to construct 'canonical Frobenius liftings', it is necessary to extract the essential 'abstract, combinatorial content' of the scheme-theoretically formulated HodgeArakelov theory of [Mzk3, Mzk4]. In fact, certain aspects of such an 'extraction process' are achieved precisely by applying the theory of Frobenioids, as is done in a sequel to this paper and [Mzk15] - namely, [Mzk16].

Here, we pause to observe that to pass from the geometry of schemes to, say, the geometry of Frobenioids amounts to a certain 'partial dismantling of scheme theory', i.e., to 'forgetting' a certain portion of scheme theory. As discussed above, one wants to execute such a 'partial dismantling of scheme theory' precisely in order to allow the construction of such objects as a 'Frobenius morphism on number fields' which are not possible within the framework of scheme theory. On the other hand, if the dismantling process that one executes is too drastic, then there is a danger of destroying so much of the geometry of scheme theory that one is not left with a geometry that is sufficiently rich so as to allow the further development of the theory. From this point of view, one of the main themes of this paper (and [Mzk15]) consists of verifying that: 
The geometry of Frobenioids retains a substantial portion of the geometry of scheme theory and, in particular, is sufficiently rich so as to permit the execution of many geometric constructions and arguments familiar from scheme theory.

The centerpiece of this verification process is the reconstruction of the functor ' $\mathcal{C} \rightarrow \mathbb{F}_{\Phi}$ ', as discussed in Section I1. Another aspect of this verification process, which may be seen throughout the theory of the present paper, is the step-by-step translation of various schemetheoretic terms and constructions that appear in the theory of divisors and line bundles on models of finite separable extensions of a given function field or number field into purely category-theoretic language. For instance, one important example of this 'step-by-step translation' is the theory of base sections and base-Frobenius pairs developed in Section 5, which may be thought of as a sort of category-theoretic translation of the notion of the tautological section of a trivial line bundle (cf. Remark 5.6.1).

\section{I4. Étale-like versus Frobenius-like structures}

Finally, let us return to the 'main result' discussed in Section I1, i.e., the reconstruction of the functor ' $\mathcal{C} \rightarrow \mathbb{F}_{\Phi}$ '. One way to think about this result is that it is a statement to the effect that:

The structure of a ('permissible') base category $\mathcal{D}$ (e.g., the subcategory of connected objects of a Galois category) is fundamentally combinatorially different - indeed, different in a category-theoretically distinguishable fashionfrom the structure of the 'Frobenius portion' $\mathbb{F}$ of a Frobenioid.

This phenomenon may be thought of as a sort of fundamental dichotomy between types of combinatorial structures - i.e., between 'étale-like' structures which are 'indifferent to order' (cf. the finite groups that as appear as Galois groups in a Galois category) and 'Frobenius-like' structures which are 'order-conscious' (cf. the monoids ' $\mathbb{Z}_{\geq 0}$ ' and ' $\mathbb{N}_{\geq 1}$ ' that constitute the standard Frobenioid $\mathbb{F}$ ). One may also think of 'étale-like' structures as 'descent-compatible' structures, whereas 'Frobenius-like' structures are 'descent-incompatible', in the sense that compatibility with 'descent' may be thought of as a sort of violation of the 'order' constituted by 'the object upstairs' in the descent operation and the 'the object downstairs'. Relative to the theme of 'abstract combinatorialization' discussed in Section I2, the point here is that the difference between 'étale-like' and 'Frobenius-like' structures is an intrinsic structural difference, not just a matter of 'arbitrarily imposed labels motivated by scheme theory' (such as 'base category', 'divisor monoid', 'Frobenius degree', etc.)! For more on this fundamental dichotomy between 'étale-like' and 'Frobenius-like' categorical structures, we refer to Remark 3.1.3.

\section{Notation and conventions}

Sets

If $E$ is a partially ordered set, then we shall denote by

$\operatorname{Order}(E)$

the category whose objects are elements $e \in E$ and whose morphisms $e_{1} \rightarrow e_{2}$ (where $e_{1}, e_{2} \in E$ ) are the relations $e_{1} \leq e_{2}$. 


\section{Numbers}

We denote by

$$
\mathbb{N}_{\geq 1}
$$

the (discrete) multiplicative monoid of rational integers $\geq 1$ and by

\section{Primes}

the set of prime numbers. Thus, one may think of $\mathbb{N}_{\geq 1}$ as the free commutative monoid generated by $\mathfrak{P r i m e s}$.

We shall write

$$
\mathbb{R}_{>0} \stackrel{\text { def }}{=}\{a \in \mathbb{R} \mid a>0\} \subseteq \mathbb{R}_{\geq 0} \stackrel{\text { def }}{=}\{a \in \mathbb{R} \mid a \geq 0\} \subseteq \mathbb{R} .
$$

We shall refer to an element

$$
\Lambda \in\{\mathbb{Z}, \mathbb{Q}, \mathbb{R}\}
$$

as a monoid type and write $\Lambda_{>0} \stackrel{\text { def }}{=} \Lambda \cap \mathbb{R}_{>0} \subseteq \mathbb{R}, \Lambda_{\geq 0} \stackrel{\text { def }}{=} \Lambda \cap \mathbb{R}_{\geq 0} \subseteq \mathbb{R}, \stackrel{\mathbb{N}}{=} \stackrel{\mathbb{Z}_{\geq 0}}{ }$. Also, we shall refer to a monoid isomorphic to (the additive monoid) $\Lambda_{\geq 0}$ as a $\Lambda$-monoprime monoid and to a monoid which is a $\Lambda$-monoprime monoid for some $\Lambda$ as monoprime. If $M$ is a $\mathbb{Q}$-monoprime monoid, then we shall write

$$
M \otimes \mathbb{R}_{\geq 0}
$$

for the $\mathbb{R}$-monoprime monoid obtained by completing $M$ relative to the topology defined by the ordering on the monoid $M$.

We shall refer to any finite extension of the field of rational numbers as a number field.

\section{Monoids}

Observe that any (not necessarily commutative!) monoid $M$ may be thought of as a special type of category, i.e., the category with precisely one object whose endomorphisms are given by the monoid $M$.

Write Mon for the category of commutative monoids (relative to some universe fixed throughout the discussion). Let $M$ be an object of $\mathfrak{M o n}$; the monoid operation of $M$ will be written additively. We shall denote by

$$
M^{ \pm} \subseteq M
$$

the submonoid (which, in fact, forms a group) of invertible elements of $M$, by

$$
M \rightarrow M^{\text {char }} \stackrel{\text { def }}{=} M / M^{ \pm}
$$

the quotient monoid of $M$ by $M^{ \pm}$, which we shall refer to as the characteristic of $M$, and by

$$
M \rightarrow M^{\mathrm{gp}}
$$

the natural homomorphism from $M$ to its groupification $M^{\mathrm{gp}}$. Thus, $M^{\mathrm{gp}}$ is the monoid (which is, in fact, a group) given by the set of equivalence classes of pairs $(a, b) \in M \times M$, where two such pairs $\left(a_{1}, b_{1}\right)$ and $\left(a_{2}, b_{2}\right)$ are considered equivalent if $a_{1}+b_{2}+c=$ 
$b_{1}+a_{2}+c$, for some $c \in M$, and the monoid operation on this set is the monoid operation induced by the monoid operation of $M$. We shall say that $M$ is torsion-free if $M$ has no torsion elements; we shall say that $M$ is sharp if $M^{ \pm}=0$; we shall say that $M$ is integral if the natural map $M \rightarrow M^{\mathrm{gp}}$ is injective; we shall say that $M$ is saturated if every $a \in M^{\mathrm{gp}}$ for which $n \cdot a$ lies in the image of $M$ for some $n \in \mathbb{N}_{\geq 1}$ lies in the image of $M$.

Denote by

$$
M^{\mathrm{pf}}
$$

the perfection of $M$; that is to say, the inductive limit of the inductive system $I_{*}$ of monoids

$$
\cdots \rightarrow M \stackrel{n \cdot}{\rightarrow} M \rightarrow \cdots
$$

given by assigning to each element of $a \in \mathbb{N}_{\geq 1}$ a copy of $M$, which we denote by $I_{a}$, and to every two elements $a, b \in M$ such that $a$ divides $b$ the morphism $I_{a}=M \rightarrow I_{b}=M$ given by multiplication by $n \stackrel{\text { def }}{=} b / a$. Thus, the object $I_{1}$ of the inductive system $I_{*}$ determines a natural morphism

$$
M \rightarrow M^{\mathrm{pf}}
$$

which is injective if $M$ is torsion-free, integral and saturated, hence, in particular, if $M$ is sharp, integral and saturated. We shall say that $M$ is perfect if multiplication by any element of $\mathbb{N}_{\geq 1}$ on $M$ is bijective. Thus, $M^{\mathrm{pf}}$ is always perfect; $M$ is perfect if and only if the natural map $M \rightarrow M^{\mathrm{pf}}$ is an isomorphism.

Note that $M$ is saturated if and only if $M^{\text {char }}$ is saturated. We shall say that $M$ is of characteristic type if the fibers of the natural map $M \rightarrow M^{\text {char }}$ are torsors over $M^{ \pm}$. Note that if $M$ is of characteristic type, then $M$ is integral if and only if $M^{\text {char }}$ is integral. If $\phi: M_{1} \rightarrow M_{2}$ is a morphism of $\mathfrak{M o n}$, then we shall say that $\phi$ is characteristically injective if $\phi$ is injective, and, moreover, the morphism $M_{1}^{\text {char }} \rightarrow M_{2}^{\text {char }}$ induced by $\phi$ is injective.

Now suppose that $M$ is sharp, integral and saturated. If $a, b \in M$, then we shall write

$$
a \leq b
$$

if $\exists c \in M$ such that $a+c=b$ and

$$
a \preccurlyeq b
$$

if $\exists n \in \mathbb{N}_{\geq 1}$ such that $a \leq n \cdot b$. If a subset $S \subseteq M$ satisfies the property that there exists a $b \in M$ such that $a \leq b$ for all $a \in S$, then we shall say that $S$ is bounded (by $b$ ). If $S \subseteq M$ is a subset and $b \in M$, then we shall write

$$
\operatorname{Bound}_{S}(b) \stackrel{\text { def }}{=}\{a \in S \mid a \leq b\}
$$

(i.e., Bound ${ }_{S}(b)$ is the maximal subset of $S$ that is bounded by $b$ ). Observe that if $M$ is $\mathbb{R}$-monoprime, then every bounded subset $S \subseteq M$ possesses a (unique) supremum

$$
\sup (S) \in M
$$

(i.e., $S$ is bounded by $b$ if and only if $b \geq \sup (S)$ ). We shall say that $0 \neq a \in M$ is irreducible if any equation $a=b+c$ in $M$, where $b, c \in M$, implies that $b=0$ or $c=0$. We shall say that $0 \neq a \in M$ is primary if for any $M \ni b \preccurlyeq a$, where $b \neq 0$, it holds that $a \preccurlyeq b$. Denote by $\operatorname{Primary}(M)$ the set of primary elements of $M$. One verifies immediately that the relation 
' $a \preccurlyeq b$ ' (where $a, b \in \operatorname{Primary}(M)$ ) determines an equivalence relation on $\operatorname{Primary}(M)$. A $\preccurlyeq$-equivalence class of elements of Primary $(M)$ will be referred to as a prime of $M$. (Note that this notion of a 'prime' differs from the conventional notion of a 'prime ideal' of $M$.) Denote by

\section{Prime $(M)$}

the set of primes of $M$. If $\mathfrak{p} \in \operatorname{Prime}(M)$, then we shall denote by

$$
M_{\mathfrak{p}} \subseteq M
$$

the submonoid generated by the elements contained in the subset $\mathfrak{p} \subseteq M$. Note that each subset $\mathfrak{p} \subseteq M$, where $\mathfrak{p} \in \operatorname{Prime}(M)$, is closed under multiplication by elements of $\mathbb{N}_{\geq 1}$, and that

$$
\begin{gathered}
\operatorname{Primary}\left(M^{\mathrm{pf}}\right)=\left\{a \in M^{\mathrm{pf}} \mid \exists n \in \mathbb{N}_{\geq 1} \text { such that } n \cdot a \in \operatorname{Primary}(M)\right\} \\
\operatorname{Primary}(M)=\operatorname{Primary}\left(M^{\mathrm{pf}}\right) \cap M \\
\operatorname{Prime}(M) \stackrel{\sim}{\rightarrow} \operatorname{Prime}\left(M^{\mathrm{pf}}\right)
\end{gathered}
$$

(where we regard $M$ as a subset of $M^{\mathrm{pf}}$ via the natural inclusion). Finally, we observe that the relation ' $\leq$ ' on elements of $M$ determines a category

$$
\operatorname{Order}(M)
$$

(via the partially ordered set structure on $M$ determined by ' $\leq$ ').

\section{Topological groups}

Let $G$ be a Hausdorff topological group and let $H \subseteq G$ be a closed subgroup. Let us write

$$
Z_{G}(H) \stackrel{\text { def }}{=}\{g \in G \mid g \cdot h=h \cdot g, \forall h \in H\}
$$

for the centralizer of $H$ in $G$.

If $\Pi$ is a profinite group, then we shall write

$$
\mathcal{B}(\Pi)
$$

for the category whose objects are finite sets equipped with a continuous $\Pi$-action and whose morphisms are morphisms of $\Pi$-sets. Thus, $\mathcal{B}(\Pi)$ is a Galois category, or, in the terminology of [Mzk7], a connected anabelioid. If $Z_{\Pi}(H)=\{1\}$ for every open subgroup $H \subseteq \Pi$, then we shall say that $\Pi$ is slim.

\section{Categories}

Let $\mathcal{C}$ be a category. We shall denote the collection of objects (respectively, arrows) of $\mathcal{C}$ by

$$
\operatorname{Ob}(\mathcal{C}) \quad(\text { respectively, } \operatorname{Arr}(\mathcal{C}))
$$

The opposite category to $\mathcal{C}$ will be denoted by $\mathcal{C}^{\text {opp }}$. A category with precisely one object will be referred to as a one-object category; a category with precisely one morphism (which is 
necessarily the identity morphism of the unique object of such a category) will be referred to as a one-morphism category. Thus, a one-morphism category is always a one-object category.

If $A \in \mathrm{Ob}(\mathcal{C})$ is an object of $\mathcal{C}$, then we shall denote by

$$
\mathcal{C}_{A}
$$

the category whose objects are morphisms $B \rightarrow A$ of $\mathcal{C}$ and whose morphisms (from an object $B_{1} \rightarrow A$ to an object $B_{2} \rightarrow A$ ) are $A$-morphisms $B_{1} \rightarrow B_{2}$ in $\mathcal{C}$ and we shall denote by

$$
{ }_{A} \mathcal{C}
$$

the category whose objects are morphisms $A \rightarrow B$ of $\mathcal{C}$ and whose morphisms (from an object $A \rightarrow B_{1}$ to an object $A \rightarrow B_{2}$ ) are morphisms $B_{1} \rightarrow B_{2}$ in $\mathcal{C}$ that are compatible with the given arrows $A \rightarrow B_{1}$ and $A \rightarrow B_{2}$. Thus, we have a natural functor

$$
\left(j_{A}\right) !: \mathcal{C}_{A} \rightarrow \mathcal{C}
$$

(given by forgetting the structure morphism to $A$ ). Similarly, if $f: A \rightarrow B$ is a morphism in $\mathcal{C}$, then $f$ defines a natural functor

$$
f_{!}: \mathcal{C}_{A} \rightarrow \mathcal{C}_{B}
$$

by mapping an arrow (i.e., an object of $\left.\mathcal{C}_{A}\right) C \rightarrow A$ to the object of $\mathcal{C}_{B}$ given by the composite $C \rightarrow A \rightarrow B$ with $f$. We shall call an object $A \in \mathrm{Ob}(\mathcal{C})$ terminal (respectively, pseudoterminal) if for every object $B \in \mathrm{Ob}(\mathcal{C})$, there exists a unique arrow (respectively, there exists a (not necessarily unique!) arrow) $B \rightarrow A$ in $\mathcal{C}$.

We shall say that two arrows of a category are co-objective if their domains and codomains coincide.

We shall say that an arrow $\beta: B \rightarrow A$ of a category $\mathcal{C}$ is fiberwise-surjective if, for every arrow $\gamma: C \rightarrow A$ of $\mathcal{C}$, there exist arrows $\delta_{B}: D \rightarrow B$ and $\delta_{C}: D \rightarrow C$ such that $\beta \circ \delta_{B}=\gamma \circ \delta_{C}$. An arrow of a category which is a fiberwise-surjective monomorphism will be referred to as an FSM-morphism. One verifies immediately that every composite of FSMmorphisms is again an FSM-morphism. A category $\mathcal{C}$ which satisfies the property that every FSM-morphism of $\mathcal{C}$ is, in fact, an isomorphism will be referred to as a category of FSM-type.

Let $\mathcal{C}$ be a category; $A \in \mathrm{Ob}(\mathcal{C})$. Write

$$
\operatorname{End}_{\mathcal{C}}(A), \quad \operatorname{Aut}_{\mathcal{C}}(A)
$$

for the monoids of endomorphisms and automorphisms of $A$ in $\mathcal{C}$, respectively. We shall say that an endomorphism $\alpha \in \operatorname{End}_{\mathcal{C}}(A)$ of $\mathcal{C}$ is a sub-automorphism if there exists an arrow $\phi: B \rightarrow A$ of $\mathcal{C}$ and an automorphism $\beta \in \operatorname{Aut}_{\mathcal{C}}(B)$ such that $\phi \circ \beta=\alpha \circ \phi$; write

$$
\left(\operatorname{Aut}_{\mathcal{C}}(A) \subseteq\right) \operatorname{Aut}_{\mathcal{C}}^{\mathrm{sub}}(A) \subseteq \operatorname{End}_{\mathcal{C}}(A)
$$

for the subset of $\operatorname{End}_{\mathcal{C}}(A)$ determined by the sub-automorphisms of $A$. We shall say that $A$ is Aut-saturated (respectively, Aut ${ }^{\text {sub }}$-saturated; of Aut-type) if $\operatorname{Aut}_{\mathcal{C}}(A)=\operatorname{Aut}_{\mathcal{C}}^{\mathrm{sub}}(A)$ (respectively, $\operatorname{Aut}_{\mathcal{C}}^{\mathrm{sub}}(A)=\operatorname{End}_{\mathcal{C}}(A) ; \operatorname{Aut}_{\mathcal{C}}(A)=\operatorname{End}_{\mathcal{C}}(A)$ ). If every object of $\mathcal{C}$ is Autsaturated (respectively, Aut ${ }^{\text {sub }}$-saturated; of Aut-type), then we shall say that $\mathcal{C}$ is Autsaturated (respectively, Aut ${ }^{\text {sub }}$-saturated; of Aut-type). We shall say that an arrow $A \rightarrow B$ of $\mathcal{C}$ is an End-equivalence if there exists an arrow $B \rightarrow A$ in $\mathcal{C}$. 
We shall refer to a natural transformation between functors all of whose component morphisms are isomorphisms as an isomorphism between the functors in question. If $\phi$ : $\mathcal{C}_{1} \rightarrow \mathcal{C}_{2}$ is a functor between categories $\mathcal{C}_{1}, \mathcal{C}_{2}$, then we shall denote by $\operatorname{Aut}(\phi)$, or, when there is no fear of confusion,

$$
\operatorname{Aut}\left(\mathcal{C}_{1} \rightarrow \mathcal{C}_{2}\right)
$$

the group of automorphisms of the functor $\phi$, and by $\operatorname{End}(\phi)$, or, when there is no fear of confusion,

$$
\operatorname{End}\left(\mathcal{C}_{1} \rightarrow \mathcal{C}_{2}\right)
$$

the monoid of natural transformations from the functor $\phi$ to itself. We shall say that $\phi$ is rigid if $\operatorname{Aut}(\phi)$ is trivial. A category $\mathcal{C}$ will be called slim if the natural functor $\mathcal{C}_{A} \rightarrow \mathcal{C}$ is rigid, for every $A \in \operatorname{Ob}(\mathcal{C})$. We recall that if $\Pi$ is a profinite group, then $\Pi$ is slim if and only if the category $\mathcal{B}(\Pi)$ is slim (cf. [Mzk7, Corollary 1.1.6]).

A diagram of functors between categories will be called 1-commutative if the various composite functors in question are isomorphic. When such a diagram 'commutes in the literal sense' we shall say that it 0-commutes. Note that when a diagram in which the various composite functors are all rigid '1-commutes', it follows from the rigidity hypothesis that any isomorphism between the composite functors in question is necessarily unique. Thus, to state that such a diagram 1-commutes does not result in any 'loss of information' by comparison to the datum of a specific isomorphism between the various composites in question.

A category $\mathcal{C}$ will be called a skeleton if any two isomorphic objects of $\mathcal{C}$ are, in fact, equal. A skeletal subcategory of a category $\mathcal{C}$ is a full subcategory $\mathcal{S} \subseteq \mathcal{C}$ such that $\mathcal{S}$ is a skeleton, and, moreover, the inclusion functor $\mathcal{S} \hookrightarrow \mathcal{C}$ is an equivalence of categories.

We shall say that a non-empty (i.e., non-initial) object $A \in \mathrm{Ob}(\mathcal{C})$ is connected if it is not isomorphic to the coproduct of two non-empty objects of $\mathcal{C}$. We shall say that an object $A \in \operatorname{Ob}(\mathcal{C})$ is mobile if there exists an object $B \in \operatorname{Ob}(\mathcal{C})$ such that the $\operatorname{set} \operatorname{Hom}_{\mathcal{C}}(A, B)$ has cardinality $\geq 2$ (i.e., the diagonal from this set to the product of this set with itself is not bijective). We shall say that an object $A \in \mathrm{Ob}(\mathcal{C})$ is quasi-connected if it is either immobile (i.e., not mobile) or connected. Thus, connected objects are always quasi-connected. We shall say that a category $\mathcal{C}$ is totally (respectively, almost totally) epimorphic if every morphism in $\mathcal{C}$ whose domain is arbitrary (respectively, non-empty) and whose codomain is arbitrary (respectively, connected) is an epimorphism.

We shall say that $\mathcal{C}$ is of finitely (respectively, countably) connected type if it is closed under formation of finite (respectively, countable) coproducts; every object of $\mathcal{C}$ is a coproduct of a finite (respectively, countable) collection of connected objects; and, moreover, all finite (respectively, countable) coproducts $\amalg A_{i}$ in the category satisfy the condition that the natural map

$$
\coprod \operatorname{Hom}_{\mathcal{C}}\left(B, A_{i}\right) \rightarrow \operatorname{Hom}_{\mathcal{C}}\left(B, \coprod A_{i}\right)
$$

is bijective, for all connected $B \in \mathrm{Ob}(\mathcal{C})$. If $\mathcal{C}$ is of finitely or countably connected type, then every non-empty object of $\mathcal{C}$ is mobile; in particular, a non-empty object of $\mathcal{C}$ is connected if and only if it is quasi-connected.

If a mobile object $A \in \mathrm{Ob}(\mathcal{C})$ satisfies the condition that every morphism in $\mathcal{C}$ whose domain is non-empty and whose codomain is $A$ is an epimorphism, then $A$ is connected. 
(Indeed, $C_{1} \amalg C_{2} \stackrel{\sim}{\rightarrow} A$, where $C_{1}, C_{2}$ are non-empty, implies that the composite map

$$
\begin{aligned}
\operatorname{Hom}_{\mathcal{C}}(A, B) & \hookrightarrow \operatorname{Hom}_{\mathcal{C}}(A, B) \times \operatorname{Hom}_{\mathcal{C}}(A, B) \hookrightarrow \operatorname{Hom}_{\mathcal{C}}\left(C_{1}, B\right) \times \operatorname{Hom}_{\mathcal{C}}\left(C_{2}, B\right) \\
& =\operatorname{Hom}_{\mathcal{C}}\left(C_{1} \coprod C_{2}, B\right) \stackrel{\sim}{\rightarrow} \operatorname{Hom}_{\mathcal{C}}(A, B)
\end{aligned}
$$

is bijective, for all $B \in \mathrm{Ob}(\mathcal{C})$.) In particular, it follows that if $\mathcal{C}$ is a totally epimorphic category, then every object of $\mathcal{C}$ is quasi-connected.

If $\mathcal{C}$ is a category of finitely or countably connected type, then we shall write

$$
\mathcal{C}^{0} \subseteq \mathcal{C}
$$

for the full subcategory of connected objects. (Note, however, that in general, objects of $\mathcal{C}^{0}$ are not necessarily connected - or even quasi-connected - as objects of $\mathcal{C}^{0}$ !) On the other hand, if, in addition, $\mathcal{C}$ is almost totally epimorphic, then $\mathcal{C}^{0}$ is totally epimorphic (so every object of $\mathcal{C}$ is quasi-connected).

If $\mathcal{C}$ is a category, then we shall write

$$
\left.\mathcal{C}^{\perp} \quad \text { (respectively, } \mathcal{C}^{\top}\right)
$$

for the category formed from $\mathcal{C}$ by taking arbitrary 'formal' (possibly empty) finite (respectively, countable) coproducts of objects in $\mathcal{C}$. That is to say, we define the 'Hom' of $\mathcal{C}^{\perp}$ (respectively, $\mathcal{C}^{\top}$ ) by the formula

$$
\operatorname{Hom}\left(\coprod_{i} A_{i}, \coprod_{j} B_{j}\right) \stackrel{\text { def }}{=} \prod_{i} \coprod_{j} \operatorname{Hom}_{\mathcal{C}}\left(A_{i}, B_{j}\right)
$$

(where the $A_{i}, B_{j}$ are objects of $\mathcal{C}$ ). Thus, $\mathcal{C}^{\perp}$ (respectively, $\mathcal{C}^{\top}$ ) is a category of finitely (respectively, countably) connected type. Note that objects of $\mathcal{C}$ define connected objects of $\mathcal{C}^{\perp}$ or $\mathcal{C}^{\top}$. Moreover, there are natural (up to isomorphism) equivalences of categories

$$
\left(\mathcal{C}^{\perp}\right)^{0} \stackrel{\sim}{\rightarrow} \mathcal{C}, \quad\left(\mathcal{C}^{\top}\right)^{0} \stackrel{\sim}{\rightarrow} \mathcal{C}, \quad\left(\mathcal{D}^{0}\right)^{\perp} \stackrel{\sim}{\rightarrow} \mathcal{D}, \quad\left(\mathcal{E}^{0}\right)^{\top} \stackrel{\sim}{\rightarrow} \mathcal{E}
$$

for $\mathcal{D}$ (respectively, $\mathcal{E}$ ) a category of finitely connected type (respectively, category of countably connected type). If $\mathcal{C}$ is a totally epimorphic category, then $\mathcal{C}^{\perp}$ (respectively, $\mathcal{C}^{\top}$ ) is an almost totally epimorphic category of finitely (respectively, countably) connected type.

In particular, the operations ' 0 ', ' $\perp$ ' (respectively, ' $T$ ') define one-to-one correspondences (up to equivalence) between the totally epimorphic categories and the almost totally epimorphic categories of finitely (respectively, countably) connected type.

We observe in passing that if $\mathcal{C}$ is a totally epimorphic category and $\alpha \circ \beta$ (where $\alpha, \beta \in \operatorname{Arr}(\mathcal{C}))$ is an isomorphism, then $\alpha, \beta$ are isomorphisms.

If $\mathcal{C}$ is a (small) category, then we shall write $\mathbb{G}(\mathcal{C})$ for the graph associated to $\mathcal{C}$. This graph is the graph with precisely one vertex for each object of $\mathcal{C}$ and precisely one edge for each arrow of $\mathcal{C}$ (joining the vertices corresponding to the domain and codomain of the arrow). We shall refer to the full subcategories of $\mathcal{C}$ determined by the objects and arrows that compose a connected component of the graph $\mathbb{G}(\mathcal{C})$ as a connected component of $\mathcal{C}$. In particular, we shall say that $\mathcal{C}$ is connected if $\mathbb{G}(\mathcal{C})$ is connected. (Note that by working with respect to some 'sufficiently large' enveloping universe, it makes sense to speak of a category which is not necessarily small as being connected.) 
Given two arrows $f_{i}: A_{i} \rightarrow B_{i}$ (where $i=1,2$ ) in a category $\mathcal{C}$, we shall refer to a commutative diagram

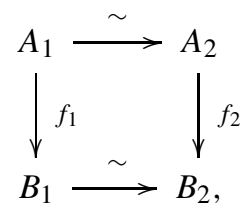

where the horizontal arrows are isomorphisms in $\mathcal{C}$, as an abstract equivalence from $f_{1}$ to $f_{2}$. If there exists an abstract equivalence from $f_{1}$ to $f_{2}$, then we shall say that $f_{1}, f_{2}$ are abstractly equivalent.

If $\mathcal{C}_{1}, \mathcal{C}_{2}$ and $\mathcal{D}$ are categories, and

$$
\Phi_{1}: \mathcal{C}_{1} \rightarrow \mathcal{D} ; \quad \Phi_{2}: \mathcal{C}_{2} \rightarrow \mathcal{D}
$$

are functors, then we define the ' $C F P$ ', i.e., 'categorical fiber product',

$$
\mathcal{C}_{1} \times{ }_{\mathcal{D}} \mathcal{C}_{2}
$$

of $\mathcal{C}_{1}, \mathcal{C}_{2}$ over $\mathcal{D}$ to be the category whose objects are triples

$$
\left(A_{1}, A_{2}, \alpha: \Phi_{1}\left(A_{1}\right) \stackrel{\sim}{\rightarrow} \Phi_{2}\left(A_{2}\right)\right),
$$

where $A_{i} \in \operatorname{Ob}\left(\mathcal{C}_{i}\right)$ (for $i=1,2$ ); $\alpha$ is an isomorphism of $\mathcal{D}$; and whose morphisms

$$
\left(A_{1}, A_{2}, \alpha: \Phi_{1}\left(A_{1}\right) \stackrel{\sim}{\rightarrow} \Phi_{2}\left(A_{2}\right)\right) \rightarrow\left(B_{1}, B_{2}, \beta: \Phi_{1}\left(B_{1}\right) \stackrel{\sim}{\rightarrow} \Phi_{2}\left(B_{2}\right)\right)
$$

are pairs of morphisms $\gamma_{i}: A_{i} \rightarrow B_{i}\left(\right.$ in $\mathcal{C}_{i}$, for $\left.i=1,2\right)$ such that $\beta \circ \Phi_{1}\left(\gamma_{1}\right)=\Phi_{2}\left(\gamma_{2}\right) \circ \alpha$. One verifies easily that if $\Phi_{2}$ is an equivalence, then the natural projection functor

$$
\mathcal{C}_{1} \times{ }_{\mathcal{D}} \mathcal{C}_{2} \rightarrow \mathcal{C}_{1}
$$

is also an equivalence.

Let $\mathcal{C}$ be a category, $\mathcal{S}$ a collection of arrows in $\mathcal{C}$ and $\phi \in \operatorname{Arr}(\mathcal{C})$. Then we shall say that $\phi$ is minimal-adjoint to $\mathcal{S}$ (respectively, minimal-coadjoint to $\mathcal{S}$; mid-adjoint to $\mathcal{S}$ ) if every factorization $\phi=\alpha \circ \beta$ (respectively, $\phi=\beta \circ \alpha ; \phi=\alpha \circ \beta \circ \gamma$ ) of $\phi$ in $\mathcal{C}$ such that $\beta$ lies in $\mathcal{S}$ satisfies the property that $\beta$ is, in fact, an isomorphism. If $\phi$ admits a factorization $\phi=\alpha \circ \beta \circ \gamma$ in $\mathcal{C}$, then we shall say that $\beta$ is subordinate to $\phi$. If $\phi$ is not an isomorphism, but, for every factorization $\phi=\alpha \circ \beta$ in $\mathcal{C}$, it holds that either $\alpha$ or $\beta$ is an isomorphism, then we shall say that $\phi$ is irreducible. We shall refer to an FSM-morphism which is irreducible as an FSMI-morphism. Thus, a category of FSM-type does not contain any FSMI-morphisms.

We shall say that a category $\mathcal{C}$ is of FSMFF-type (i.e., 'FSM-finitely factorizable type') if the following two conditions hold: (a) every FSM-morphism of $\mathcal{C}$ which is not an isomorphism factors as a composite of finitely many FSMI-morphisms; (b) for every $A \in \operatorname{Ob}(\mathcal{C})$, there exists a natural number $N$ such that for every composite

$$
\phi_{n} \circ \phi_{n-1} \circ \cdots \circ \phi_{2} \circ \phi_{1}
$$

of FSMI-morphisms $\phi_{1}, \ldots, \phi_{n}$ such that the domain of $\phi_{1}$ is equal to $A$, it holds that $n \leq N$. Thus, if $\mathcal{C}$ is of FSM-type, then it is of FSMFF-type. Also, we observe that (by condition (b)) no endomorphism of an object of a category of FSMFF-type is an FSMI-morphism. 
If $\mathcal{C}$ is a totally epimorphic category, $A \in \mathrm{Ob}(\mathcal{C})$, and $G \subseteq \operatorname{Aut}_{\mathcal{C}}(A)$ is a subgroup, then we shall say that an arrow $\phi: A \rightarrow B$ of $\mathcal{C}$ is a categorical quotient of $A$ by $G$ if the following conditions hold: (a) $\phi \circ \gamma=\phi$, for all $\gamma \in G$; (b) for every morphism $\psi: A \rightarrow C$ such that $\psi \circ \gamma=\psi$ for all $\gamma \in G$, there exists a unique morphism $\psi^{\prime}: B \rightarrow C$ such that $\psi=\psi^{\prime} \circ \phi$. If $\phi: A \rightarrow B$ is a categorical quotient of $A$ by $G$, then we shall say that $A \rightarrow B$ is mono-minimal if the following condition holds: for every factorization $\phi=\phi^{\prime} \circ \zeta$, where $\zeta: A \rightarrow A^{\prime}$ is a monomorphism such that there exists a subgroup $G^{\prime} \subseteq \operatorname{Aut}_{\mathcal{C}}\left(A^{\prime}\right)$, together with an isomorphism $G \stackrel{\sim}{\rightarrow} G^{\prime}$ that is compatible, relative to $\zeta$, with the respective actions of $G, G^{\prime}$ on $A, A^{\prime}$ (which implies, by total epimorphicity, that $\phi^{\prime}: A^{\prime} \rightarrow B$ is a categorical quotient of $A^{\prime}$ by $G^{\prime}$ ), it holds that $\zeta$ is an isomorphism. Thus, (by total epimorphicity) it follows that an isomorphism is always a mono-minimal categorical quotient of its domain by the trivial group.

If $\mathcal{C}$ is a category, then we shall say that $A \in \mathrm{Ob}(\mathcal{C})$ is an anchor if there only exist finitely many isomorphism classes of objects of ${ }_{A} \mathcal{C}$ that arise from irreducible arrows $A \rightarrow B$. We shall say that $A \in \operatorname{Ob}(\mathcal{C})$ is a subanchor if there exists an arrow $A \rightarrow B$, where $B$ is an anchor. If $\mathcal{C}$ is a totally epimorphic category, then we shall say that $A \in \mathrm{Ob}(\mathcal{C})$ is an iso-subanchor if there exist a subanchor $B \in \operatorname{Ob}(\mathcal{C})$, a subgroup $G \subseteq \operatorname{Aut}_{\mathcal{C}}(B)$ and a morphism $B \rightarrow A$ (in $\mathcal{C}$ ) which is a mono-minimal categorical quotient of $B$ by $G$.

\section{Definitions and first properties}

In this section, we discuss the notion of a Frobenioid, which may be thought of as a category whose internal structure behaves roughly like that of an 'elementary Frobenioid'. An 'elementary Frobenioid' is, in essence, a sort of semi-direct product of the multiplicative monoid $\mathbb{N}_{\geq 1}$ (which is to be thought of as a 'Frobenius action') with a system of monoids (which are roughly of the sort that appear in the theory of log structures) on a 'base category' (a category which behaves roughly like a Galois category).

We begin by introducing the fundamental notions of 'elementary Frobenioids' and 'preFrobenioids'.

Definition 1.1.

(i) We shall say that $M \in \mathrm{Ob}(\mathfrak{M o n})$ is pre-divisorial if it is integral, saturated and of characteristic type (cf. Section 0). Suppose that $M$ is pre-divisorial. Then we shall say that $M$ is group-like if $M^{\text {char }}$ is zero; we shall say that $M$ is divisorial if $M$ is sharp (cf. Section 0). (Thus, if $M$ is pre-divisorial, then $M^{\text {char }}$ is divisorial.) If $\alpha$ is an endomorphism of a pre-divisorial monoid $M \in \mathrm{Ob}(\mathfrak{M o n})$, then we shall say that $\alpha$ is non-dilating if the endomorphism $\alpha^{\text {char }}$ of $M^{\text {char }}$ induced by $\alpha$ is the identity endomorphism of $M^{\text {char }}$ whenever $\alpha^{\text {char }}(a) \preccurlyeq a$ for all primary (cf. Section 0) $a \in M^{\text {char }}$.

(ii) Let $\mathcal{D}$ be a category. Then we shall refer to a contravariant functor

$$
\Phi: \mathcal{D} \rightarrow \mathfrak{M o n}
$$

as a monoid on $\mathcal{D}$ if the following conditions are satisfied: (a) every morphism of monoids $\alpha^{*}: \Phi(A) \rightarrow \Phi(B)$ induced by a morphism $\alpha: B \rightarrow A$ of $\mathcal{D}$ is characteristically injective (cf. Section 0); (b) if $\alpha$ is an FSM-morphism (cf. Section 0) of $\mathcal{D}$, then $\alpha^{*}: \Phi(A) \rightarrow \Phi(B)$ is 
an isomorphism of monoids. If, moreover, every monoid $\Phi(A)$, as $A$ ranges over the objects of $\mathcal{D}$, (respectively, some monoid $\Phi(A)$, where $A \in \operatorname{Ob}(\mathcal{D})$ ) satisfies some property of monoids (e.g., is pre-divisorial, sharp, etc.), then we shall say that $\Phi$ (respectively, $A$ ) satisfies this property. Note that if $\Phi$ is a monoid on $\mathcal{D}$, then $\Phi$ determines monoids ' $\Phi^{\text {char }}$, ' $\Phi$ gp' and $\Phi^{\mathrm{pf}}$, on $\mathcal{D}$ (i.e., by assigning $A \mapsto \Phi(A)^{\text {char }}, A \mapsto \Phi(A)^{\mathrm{gp}}$ and $A \mapsto \Phi(A)^{\mathrm{pf}}$ ), which we shall refer to, respectively, as the characteristic, groupification and perfection of $\Phi$. If $\Phi$ is pre-divisorial, then we shall say that $\Phi$ is non-dilating if the endomorphisms of $\Phi(A)$, where $A \in \operatorname{Ob}(\mathcal{D})$, induced by endomorphisms $\in \operatorname{End}_{\mathcal{D}}(A)$ are non-dilating.

(iii) Let $\Phi$ be a monoid on a category $\mathcal{D}$. Then we shall refer to the category

$$
\mathbb{F}_{\Phi}
$$

as the elementary Frobenioid associated to $\Phi$ defined as follows. The objects of $\mathbb{F}_{\Phi}$ are the objects of $\mathcal{D}$. If $A, B \in \mathrm{Ob}\left(\mathbb{F}_{\Phi}\right)$, whose respective images in $\mathcal{D}$ we denote by $A_{\mathcal{D}}, B_{\mathcal{D}} \in$ $\operatorname{Ob}(\mathcal{D})$, then a morphism $\phi: A \rightarrow B$ of $\mathbb{F}_{\Phi}$ is defined to be a collection of data

$$
\left(\phi_{\mathcal{D}}, Z_{\phi}, n_{\phi}\right)
$$

where $\phi_{\mathcal{D}}: A_{\mathcal{D}} \rightarrow B_{\mathcal{D}}$ is a morphism of $\mathcal{D} ; \quad Z_{\phi} \in \Phi\left(A_{\mathcal{D}}\right) ; n_{\phi} \in \mathbb{N}_{\geq 1}$. Here, $\phi_{\mathcal{D}}$ (respectively, $A_{\mathcal{D}}$ ) will be referred to as the projection $\operatorname{Base}(\phi)$ (respectively, $\operatorname{Base}(A)$ ) of $\phi$ (respectively, $A$ ) to $\mathcal{D}, Z_{\phi}$ as the zero divisor $\operatorname{Div}(\phi)$ of $\phi$ and $n_{\phi}$ as the Frobenius degree $\operatorname{deg}_{\mathrm{Fr}}(\phi)$ of $\phi$. If $C_{\mathcal{D}} \stackrel{\text { def }}{=} \operatorname{Base}(C) \in \mathrm{Ob}(\mathcal{D})$, then the composite of two morphisms

$$
\phi=\left(\phi_{\mathcal{D}}, Z_{\phi}, n_{\phi}\right): A \rightarrow B, \quad \psi=\left(\psi_{\mathcal{D}}, Z_{\psi}, n_{\psi}\right): B \rightarrow C
$$

is given as

$$
\psi \circ \phi=\left(\psi_{\mathcal{D}} \circ \phi_{\mathcal{D}}, \phi_{\mathcal{D}}^{*}\left(Z_{\psi}\right)+n_{\psi} \cdot Z_{\phi}, n_{\psi} \cdot n_{\phi}\right): A \rightarrow C .
$$

Observe that the assignment $\Phi \mapsto \mathbb{F}_{\Phi}$ is functorial with respect to homomorphisms of functors (on $\mathcal{D}$ ) valued in monoids $\Phi \rightarrow \Phi^{\prime}$; also, we have a natural projection functor

$$
\mathbb{F}_{\Phi} \rightarrow \mathcal{D}
$$

We shall refer to the $\mathcal{D}$ as the base category of $\mathbb{F}_{\Phi}$. If $M \in \mathrm{Ob}(\mathfrak{M o n})$, then observe that the elementary Frobenioid $\mathbb{F}_{\Phi_{M}}$ associated to the functor $\Phi_{M}$ on any one-morphism (cf. Section 0) category that assigns to the unique object of the category the monoid $M$ is itself a one-object (cf. Section 0) category, whose endomorphism monoid we shall denote by $\mathbb{F}_{M}$ and refer to as the elementary Frobenioid associated to $M$. (Thus, the notation ' $F$ ' denotes a category (respectively, monoid) when the subscript ' $\square$ ' is a functor (respectively, monoid).) More explicitly, the underlying set of $\mathbb{F}_{M}$ is the product

$$
M \times \mathbb{N}_{\geq 1}
$$

equipped with the monoid structure given as follows: if $a_{1}, a_{2} \in M, n_{1}, n_{2} \in \mathbb{N}_{\geq 1}$, then $\left(a_{1}, n_{1}\right) \cdot\left(a_{2}, n_{2}\right)=\left(a_{1}+n_{1} \cdot a_{2}, n_{1} \cdot n_{2}\right)$. Also, we shall write $\mathbb{F} \stackrel{\text { def }}{=} \mathbb{F}_{\mathbb{Z}_{\geq 0}}$ and refer to $\mathbb{F}$ as the standard Frobenioid. 
(iv) Let $\mathcal{D}, \Phi, \mathbb{F}_{\Phi}$ be as in (iii); $\mathcal{C}$ a category. Assume further that $\Phi$ is divisorial, and that $\mathcal{C}, \mathcal{D}$ are connected, totally epimorphic categories (cf. Section 0 ). Then we shall refer to a (covariant) functor

$$
\mathcal{C} \rightarrow \mathbb{F}_{\Phi}
$$

as a pre-Frobenioid structure on $\mathcal{C}$. The natural projection functor $\mathbb{F}_{\Phi} \rightarrow \mathcal{D}$ thus restricts to a natural projection functor

$$
\mathcal{C} \rightarrow \mathcal{D}
$$

on $\mathcal{C}$; similarly, the operations 'Base(-)', 'Div(-)' and ' $\operatorname{deg}_{\mathrm{Fr}}(-)$ ' on $\mathbb{F}_{\Phi}$ restrict to operations on $\mathcal{C}$ which (by abuse of notation) we shall denote by the same notation. We shall refer to the $\mathcal{D}$ as the base category of $\mathcal{C}$. By abuse of notation, we shall often regard $\Phi$ as a functor on $\mathcal{C}$ (i.e., by composing the original functor $\Phi$ with the natural projection functor $\mathcal{C} \rightarrow \mathcal{D}$ ) and apply similar terminology to objects of $\mathcal{C}$ and ' $\Phi$ as a functor on $\mathcal{C}$ ' to the terminology applied to objects of $\mathcal{D}$ and ' $\Phi$ as a functor on $\mathcal{D}$ ' (cf. (ii)). We shall refer to a category $\mathcal{C}$ equipped with a pre-Frobenioid structure $\mathcal{C} \rightarrow \mathbb{F}_{\Phi}$ as a pre-Frobenioid and to the monoid $\Phi$ as the divisor monoid of the pre-Frobenioid.

Remark 1.1.1. If $\phi \circ \psi$ is a composite of morphisms $\phi$ and $\psi$ of a pre-Frobenioid, then the operations 'Base(-)', 'Div(-)' and ' $\operatorname{deg}_{\mathrm{Fr}}(-)$ ' behave in the following way under composition:

$$
\begin{gathered}
\operatorname{Base}(\phi \circ \psi)=\operatorname{Base}(\phi) \circ \operatorname{Base}(\psi), \\
\operatorname{Div}(\phi \circ \psi)=(\operatorname{Base}(\psi))^{*}(\operatorname{Div}(\phi))+\operatorname{deg}_{\mathrm{Fr}}(\phi) \cdot \operatorname{Div}(\psi), \\
\operatorname{deg}_{\mathrm{Fr}}(\phi \circ \psi)=\operatorname{deg}_{\mathrm{Fr}}(\phi) \cdot \operatorname{deg}_{\mathrm{Fr}}(\psi) .
\end{gathered}
$$

Indeed, this follows immediately from the definition of an elementary Frobenioid in Definition 1.1(iii).

Next, we introduce various terms to describe types of morphisms and objects in a preFrobenioid.

Definition 1.2. Let $\Phi$ be a divisorial monoid on a connected, totally epimorphic category $\mathcal{D} ; \mathcal{C} \rightarrow \mathbb{F}_{\Phi}$ a pre-Frobenioid; $\phi \in \operatorname{Arr}(\mathcal{C})$. Write $\phi: A \rightarrow B($ where $A, B \in \operatorname{Ob}(\mathcal{C})), A_{\mathcal{D}} \stackrel{\text { def }}{=}$ $\operatorname{Base}(A) \in \operatorname{Ob}(\mathcal{D})$ and $B_{\mathcal{D}} \stackrel{\text { def }}{=} \operatorname{Base}(B) \in \operatorname{Ob}(\mathcal{D})$.

(i) We shall say that $\phi$ is linear if $\operatorname{deg}_{\mathrm{Fr}}(\phi)=1$. We shall say that $\phi$ is isometric, or, alternatively, an isometry, if $\operatorname{Div}(\phi)=0$ (cf. Definition 1.1(iii)). If $\psi \in \operatorname{Arr}(\mathcal{C})$ is co-objective with $\phi$ (cf. Section 0$)$, then we shall say that $\phi$ and $\psi$ are metrically equivalent if $\operatorname{Div}(\phi)=$ $\operatorname{Div}(\psi)$.

(ii) We shall refer to $\phi$ as a base-isomorphism (respectively, base-FSM-morphism) if $\operatorname{Base}(\phi)$ is an isomorphism (respectively, FSM-morphism (cf. Section 0)) in $\mathcal{D}$. We shall refer to two objects of $\mathcal{C}$ that map to isomorphic objects of $\mathcal{D}$ as base-isomorphic. We shall refer to $\phi$ as a pull-back morphism if the natural transformation of contravariant functors on $\mathcal{C}$

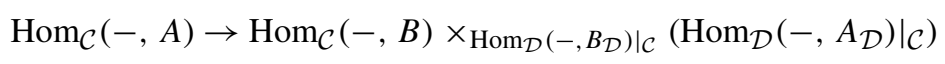

(where ' $\mid \mathcal{C}$ ' denotes the restriction of a functor on $\mathcal{D}$ to a functor on $\mathcal{C}$ via the natural projection functor $\mathcal{C} \rightarrow \mathcal{D}$ ) induced by $\phi$ is an isomorphism. If $\psi \in \operatorname{Arr}(\mathcal{C})$ is co-objective with $\phi$ 
(cf. Section 0), then we shall say that $\phi$ and $\psi$ are base-equivalent (respectively, Div-equivalent) if $\operatorname{Base}(\phi)=\operatorname{Base}(\psi)$ (respectively, $\Phi(\phi)=\Phi(\psi)$ ). If $A=B$ (i.e., $\phi$ is an endomorphism), then we shall say that $\phi$ is a base-identity (respectively, Dividentity) endomorphism if it is base-equivalent (respectively, Div-equivalent) to the identity endomorphism of $A$. Write

$$
\mathcal{O}^{\times}(A) \subseteq \operatorname{Aut}_{\mathcal{C}}(A), \quad \mathcal{O}^{\triangleright}(A) \subseteq \operatorname{End}_{\mathcal{C}}(A)
$$

for the submonoids of base-identity linear endomorphisms.

(iii) We shall say that $\phi$ is a pre-step (a term motivated by the point of view that the only possibly non-isomorphic portion of such a morphism is the 'step' constituted by a non-zero zero divisor) if it is a linear base-isomorphism. If $\phi$ is a pre-step, then we shall say that it is a step (respectively, a primary pre-step) if $\phi$ is not an isomorphism (respectively, if the zero divisor $\operatorname{Div}(\phi) \in \Phi(A)$ of $\phi$ is a primary (cf. Section 0 ) element of the monoid $\Phi(A)$ ). We shall say that $\phi$ is co-angular (a term that arises from a certain 'coincidence of angles' that occurs for co-angular morphisms in the case of Frobenioids that arise in an archimedean context - cf. [Mzk15, Definition 3.1(iii)]) if, for any factorization $\phi=\alpha \circ \beta \circ \gamma$ in $\mathcal{C}$, where $\alpha$ is linear, $\beta$ is an isometric pre-step and either $\alpha$ or $\gamma$ is a base-isomorphism, it follows that $\beta$ is an isomorphism. We shall say that $\phi$ is LB-invertible (i.e., 'line bundle-invertible' a term motivated by the isomorphism induced by such a morphism between the 'image line bundle of the domain' and 'the line bundle portion of the codomain' in the case of various Frobenioids that arise from arithmetic geometry) if it is co-angular and isometric. We shall say that $\phi$ is a morphism of Frobenius type (a term motivated by the fact that, in the case of Frobenioids that arise from arithmetic geometry, such a morphism corresponds to simply 'raising to the $n$th tensor power' for some $n \in \mathbb{N}_{\geq 1}$ ) if $\phi$ is an LB-invertible base-isomorphism. We shall say that $\phi$ is a prime-Frobenius morphism, or, alternatively, a $\operatorname{deg}_{\mathrm{Fr}}(\phi)$-Frobenius morphism, if it is a morphism of Frobenius type such that $\operatorname{deg}_{\mathrm{Fr}}(\phi) \in \mathfrak{P r i m e s}$ (cf. Section 0).

(iv) A Frobenius-ample object of $\mathcal{C}$ is defined to be an object $C$ such that for any $n \in \mathbb{N}_{\geq 1}, C$ admits an endomorphism of Frobenius degree $n$. A Frobenius-trivial object of $\mathcal{C}$ is defined to be an object $C$ such that there exists a homomorphism of monoids $\zeta: \mathbb{N}_{\geq 1} \rightarrow \operatorname{End}_{\mathcal{C}}(C)$ which satisfies the following properties: (a) the composite of $\zeta$ with the map to $\mathbb{N}_{\geq 1}$ given by the Frobenius degree is the identity on $\mathbb{N}_{\geq 1}$; (b) the endomorphisms in the image of $\zeta$ are baseidentity endomorphisms of Frobenius type. A Div-Frobenius-trivial object of $\mathcal{C}$ is defined to be an object $C$ such that there exists a homomorphism of monoids $\zeta: \mathbb{N}_{\geq 1} \rightarrow \operatorname{End}_{\mathcal{C}}(C)$ which satisfies the following properties: (a) the composite of $\zeta$ with the map to $\mathbb{N}_{\geq 1}$ given by the Frobenius degree is the identity on $\mathbb{N}_{\geq 1}$; (b) the endomorphisms in the image of $\zeta$ are Div-identity endomorphisms of Frobenius type. A universally Div-Frobenius-trivial object of $\mathcal{C}$ is defined to be an object $C$ such that for every pull-back morphism $C^{\prime} \rightarrow C$ of $\mathcal{C}$, it follows that $C^{\prime}$ is a Div-Frobenius-trivial object. A quasi-Frobenius-trivial object of $\mathcal{C}$ is defined to be an object $C$ such that for any $n \in \mathbb{N}_{\geq 1}, C$ admits a base-identity endomorphism (which is not necessarily of Frobenius type!) of Frobenius degree $n$. A sub-quasi-Frobeniustrivial object of $\mathcal{C}$ is defined to be an object $C$ such that there exists a co-angular prestep $D \rightarrow C$ in $\mathcal{C}$ such that $D$ is quasi-Frobenius trivial. A metrically trivial object of $\mathcal{C}$ is defined to be an object $C$ such that for any co-angular pre-step $C \rightarrow D$, it holds that $D$ is isomorphic to $C$. A base-trivial object of $\mathcal{C}$ is defined to be an object $C$ such that any 
object $D \in \operatorname{Ob}(\mathcal{C})$ such that $\operatorname{Base}(C) \cong \operatorname{Base}(D)$ (in $\mathcal{D}$ ) is, in fact, isomorphic to $C$. An Autample (respectively, Aut ${ }^{\text {sub }}$-ample; End-ample) object of $\mathcal{C}$ is defined to be an object $C$ such that, if we write $C_{\mathcal{D}} \stackrel{\text { def }}{=} \operatorname{Base}(C)$, then the natural map $\operatorname{Aut}_{\mathcal{C}}(C) \rightarrow \operatorname{Aut}_{\mathcal{D}}\left(C_{\mathcal{D}}\right)$ (respectively, $\left.\operatorname{Aut}_{\mathcal{C}}^{\text {sub }}(C) \rightarrow \operatorname{Aut}_{\mathcal{D}}^{\text {sub }}\left(C_{\mathcal{D}}\right) ; \operatorname{End}_{\mathcal{C}}(C) \rightarrow \operatorname{End}_{\mathcal{D}}\left(C_{\mathcal{D}}\right)\right)$ is surjective. A perfect object of $\mathcal{C}$ is defined to be an object $C$ such that for every $n \in \mathbb{N}_{\geq 1}$, it holds that every $B \in \mathrm{Ob}(\mathcal{C})$ baseisomorphic to $C$ appears as the codomain of a morphism of Frobenius type of Frobenius degree $n$, and, moreover, for every pair of morphisms of Frobenius type $\phi_{1}: B_{1} \rightarrow B_{1}^{\prime}$, $\phi_{2}: B_{2} \rightarrow B_{2}^{\prime}$ of Frobenius degree $n$, where $B_{1}, B_{2}$ are base-isomorphic to $C$, and every prestep $\psi^{\prime}: B_{1}^{\prime} \rightarrow B_{2}^{\prime}$, there exists a unique pre-step $\psi: B_{1} \rightarrow B_{2}$ such that $\psi^{\prime} \circ \phi_{1}=\phi_{2} \circ \psi$. A group-like object of $\mathcal{C}$ is defined to be an object $C$ such that $\Phi(C)=0$ (or, equivalently, $\Phi(C)$ is group-like - cf. the conventions of Definition 1.1(i), (ii) and (iv)). A Frobenius-compact object of $\mathcal{C}$ is defined to be an object $C$ such that $\mathcal{O}^{\times}(C)$ is commutative, $\mathcal{O}^{\times}(C)^{\mathrm{pf}} \neq 0$ and every element of $\operatorname{Aut}_{\mathcal{C}}(C)$ that acts on $\mathcal{O}^{\times}(C)^{\text {pf }}$ via multiplication by an element $\in \mathbb{Q}_{>0}$ in fact acts trivially on $\mathcal{O}^{\times}(C)^{\mathrm{pf}}$. A Frobenius-normalized object of $\mathcal{C}$ is defined to be an object $C$ such that if $\phi \in \operatorname{End}_{\mathcal{C}}(C)$ is a base-identity endomorphism of Frobenius degree $d \in \mathbb{N}_{\geq 1}$, and $\alpha \in \mathcal{O}^{\triangleright}(C)$, then $\alpha^{d} \circ \phi=\phi \circ \alpha$. A unit-trivial object of $\mathcal{C}$ is defined to be an object $C$ such that $\mathcal{O}^{\times}(C)=\{1\}$. An isotropic object (a term motivated by the archimedean case cf. [Mzk15, Definition 3.1(iii)]) of $\mathcal{C}$ is defined to be an object $C$ such that any isometric pre-step $C \rightarrow D$ in $\mathcal{C}$ is, in fact, an isomorphism. We shall write

$$
\mathcal{C}^{\text {istr }} \subseteq \mathcal{C}
$$

for the full subcategory of isotropic objects and

$$
\mathcal{C}^{\text {lin }} \subseteq \mathcal{C}, \quad \mathcal{C}^{\text {bs-iso }} \subseteq \mathcal{C}, \quad \mathcal{C}^{\text {pl-bk }} \subseteq \mathcal{C}
$$

for the subcategories determined, respectively, by the linear morphisms, base-isomorphisms and pull-back morphisms. We shall say that $\phi: A \rightarrow B$ is an isotropic hull (of $A$ ) if $\phi$ is an isometric pre-step, $B$ is isotropic and for every morphism $\gamma: A \rightarrow C$, where $C$ is isotropic, there exists a unique morphism $\beta: B \rightarrow C$ such that $\gamma=\beta \circ \phi$. A Frobenius-isotropic object of $\mathcal{C}$ is defined to be an object $C$ such that there exists a morphism of Frobenius type $C \rightarrow D$ such that $D$ is isotropic.

(v) If every object of $\mathcal{C}$ is Frobenius-ample (respectively, Frobenius-trivial; Div-Frobeniustrivial; universally Div-Frobenius-trivial; quasi-Frobenius-trivial; sub-quasi-Frobeniustrivial; metrically trivial; base-trivial; Aut-ample; Aut ${ }^{\text {sub }}$-ample; End-ample; perfect; grouplike; Frobenius-compact; Frobenius-normalized; unit-trivial; isotropic; Frobenius-isotropic), then we shall say that the pre-Frobenioid $\mathcal{C} \rightarrow \mathbb{F}_{\Phi}$ is of Frobenius-ample type (respectively, of Frobenius-trivial type; of Div-Frobenius-trivial type; of universally Div-Frobenius-trivial type; of quasi-Frobenius-trivial type; of sub-quasi-Frobenius-trivial type; of metrically trivial type; of base-trivial type; of Aut-ample type; of $\mathrm{Aut}^{\mathrm{sub}}$-ample type; of End-ample type; of perfect type; of group-like type; of Frobenius-compact type; of Frobenius-normalized type; of unit-trivial type; of isotropic type; of Frobenius-isotropic type). 
Remark 1.2.1. The following implications follow formally from the definitions:

pull-back morphism which is a base-isomorphism $\Longleftrightarrow$ isomorphism,

$$
\begin{gathered}
\text { base-trivial } \Longrightarrow \text { metrically trivial, } \\
\text { base-identity } \Longrightarrow \text { Div-identity, }
\end{gathered}
$$$$
\text { universally Div-Frobenius-trivial } \Longrightarrow \text { Div-Frobenius-trivial. }
$$

We are now ready to define the notion of a 'Frobenioid'.

Definition 1.3. Let $\mathcal{D}, \Phi$ and $\mathcal{C} \rightarrow \mathbb{F}_{\Phi}$ be as in Definition 1.2. Then we shall say that the pre-Frobenioid $\mathcal{C} \rightarrow \mathbb{F}_{\Phi}$ (i.e., $\mathcal{C}$ equipped with this functor) is a Frobenioid if the following conditions are satisfied.

(i) Surjectivity to the base category via pull-back morphisms.

(a) Every isomorphism class of $\mathcal{D}$ arises as the image via the natural projection functor $\mathcal{C} \rightarrow \mathcal{D}$ of an isomorphism class of a Frobenius-trivial object of $\mathcal{C}$.

(b) If $A, B \in \mathrm{Ob}(\mathcal{C}), A_{\mathcal{D}} \stackrel{\text { def }}{=} \operatorname{Base}(A), B_{\mathcal{D}} \stackrel{\text { def }}{=} \operatorname{Base}(B)$ and $\alpha: A_{\mathcal{D}} \stackrel{\sim}{\rightarrow} B_{\mathcal{D}}$ is an isomorphism, then there exist pre-steps $\phi: C \rightarrow A$ and $\psi: C \rightarrow B$ such that $\alpha=$ $\operatorname{Base}(\psi) \circ \operatorname{Base}(\phi)^{-1}$.

(c) For every $A \in \operatorname{Ob}(\mathcal{C})$, the fully faithful (cf. the isomorphism of functors appearing in the definition of a 'pull-back morphism' given in Definition 1.2(ii)) functor

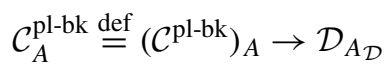

(where $A_{\mathcal{D}} \stackrel{\text { def }}{=} \operatorname{Base}(A)$ ) determined by the natural projection functor $\mathcal{C} \rightarrow \mathcal{D}$ is an equivalence of categories (cf. Section 0).

(ii) Surjectivity to $\mathbb{N}_{\geq 1}$ via morphisms of Frobenius type. For every $A \in \operatorname{Ob}(\mathcal{C}), n \in \mathbb{N}_{\geq 1}$, there exists a morphism of Frobenius type $\phi: A \rightarrow B$ in $\mathcal{C}$ of Frobenius degree $n$; moreover, if $\psi: A \rightarrow C$ is any other morphism of Frobenius type in $\mathcal{C}$ of Frobenius degree $n$, then there exists a(n) (unique - since $\mathcal{C}$ is totally epimorphic) isomorphism $\beta: B \stackrel{\sim}{\rightarrow} C$ such that $\beta \circ \phi=\psi$.

(iii) Surjectivity to the divisor monoid via co-angular morphisms.

(a) The co-angular morphisms of $\mathcal{C}$ are closed under composition.

(b) If $A^{\prime} \rightarrow A$ is a co-angular pre-step of $\mathcal{C}$, then any morphism $A^{\prime} \rightarrow A$ is co-angular.

(c) Given any co-angular pre-step $\phi: A \rightarrow B$, there exists a (uniquely determined) bijection of monoids

$$
\mathcal{O}^{\triangleright}(A) \stackrel{\sim}{\rightarrow} \mathcal{O}^{\triangleright}(B)
$$

such that $\mathcal{O}^{\triangleright}(A) \ni \alpha \mapsto \beta \in \mathcal{O}^{\triangleright}(B)$ implies $\beta \circ \phi=\phi \circ \alpha$; moreover, this bijection depends only (among the bijections induced by the various co-angular pre-steps $A \rightarrow$ $B)$ on $\operatorname{Base}(\phi)$.

(d) Denote by $\mathcal{C}^{\text {coa-pre }} \subseteq \mathcal{C}$ the subcategory determined by the co-angular pre-steps. Then the natural functors

$$
\begin{aligned}
& { }_{A} \mathcal{C}^{\text {coa-pre }} \stackrel{\text { def }}{=}{ }_{A}\left(\mathcal{C}^{\text {coa-pre }}\right) \rightarrow \operatorname{Order}(\Phi(A)), \\
& \mathcal{C}_{A}^{\text {coa-pre def }} \stackrel{\text { e }}{=}\left(\mathcal{C}^{\text {coa-pre }}\right)_{A} \rightarrow \operatorname{Order}(\Phi(A))^{\text {opp }}
\end{aligned}
$$


(obtained by assigning to an arrow $\phi: A \rightarrow B$ the element $\operatorname{Div}(\phi) \in \Phi(A)$ and to an arrow $\psi: B \rightarrow A$ the element $\left(\psi^{*}\right)^{-1}(\operatorname{Div}(\psi)) \in \Phi(A)$ (since $\psi^{*}: \Phi(A) \stackrel{\sim}{\rightarrow} \Phi(B)$ is a bijection - cf. the fact that $\psi$ is a base-isomorphism!) are equivalences of categories.

(iv) Factorization of arbitrary morphisms. Let $\phi: A \rightarrow B$ be a morphism of $\mathcal{C}$.

(a) $\phi$ admits a factorization

$$
\phi=\alpha \circ \beta \circ \gamma
$$

where $\alpha$ is an pull-back morphism, $\beta$ is a pre-step and $\gamma$ is a morphism of Frobenius type; this factorization is unique, up to replacing the triple $(\alpha, \beta, \gamma)$ by a triple of the form $\left(\alpha \circ \delta, \delta^{-1} \circ \beta \circ \epsilon, \epsilon^{-1} \circ \gamma\right)$, where $\delta, \epsilon$ are isomorphisms of $\mathcal{C}$.

(b) Every pull-back morphism of $\mathcal{C}$ is $L B$-invertible and linear.

(v) Factorization of pre-steps. Let $\phi: A \rightarrow B$ be a pre-step of $\mathcal{C}$.

(a) $\phi$ is a monomorphism.

(b) $\phi$ admits a factorization

$$
\phi=\alpha \circ \beta
$$

where $\alpha$ is an isometric pre-step and $\beta$ is a co-angular pre-step; this factorization is unique, up to replacing the pair $(\alpha, \beta)$ by a pair of the form $\left(\alpha \circ \gamma, \gamma^{-1} \circ \beta\right)$, where $\gamma$ is an isomorphism of $\mathcal{C}$.

(c) $\phi$ admits a factorization $\phi=\alpha^{\prime} \circ \beta^{\prime}$, where $\alpha^{\prime}$ is a co-angular pre-step and $\beta^{\prime}$ is an isometric pre-step; this factorization is unique, up to replacing the pair $\left(\alpha^{\prime}, \beta^{\prime}\right)$ by a pair of the form $\left(\alpha^{\prime} \circ \gamma^{\prime},\left(\gamma^{\prime}\right)^{-1} \circ \beta^{\prime}\right)$, where $\gamma^{\prime}$ is an isomorphism of $\mathcal{C}$.

(vi) Faithfulness up to units. Let $\phi, \psi: A \rightarrow B$ be base-equivalent, metrically equivalent co-angular pre-steps of $\mathcal{C}$. Then there exists a (necessarily unique) $\alpha \in \mathcal{O}^{\times}(B)$ such that $\phi=\alpha \circ \psi$.

(vii) Isotropic objects.

(a) For every $A \in \operatorname{Ob}(\mathcal{C})$, there exists a (necessarily unique, up to unique isomorphism) isotropic hull $A \rightarrow B$.

(b) If $A \in \operatorname{Ob}(\mathcal{C})$ is isotropic and $A \rightarrow C$ is a morphism of $\mathcal{C}$, then $C$ is also isotropic.

Remark 1.3.1. Note that it follows from Definition 1.3(iii)(b) and (c), that if $\mathcal{C}$ is a Frobenioid, then the monoid $\mathcal{O}^{\triangleright}(A)$ is commutative, for all $A \in \mathrm{Ob}(\mathcal{C})$.

PROPOSITION 1.4. (Co-angular and LB-invertible morphisms) Let $\Phi$ be a divisorial monoid on a connected, totally epimorphic category $\mathcal{D}$ let $\mathcal{C} \rightarrow \mathbb{F}_{\Phi}$ be a pre-Frobenioid and let $\phi: A \rightarrow B$ be a morphism of $\mathcal{C}$. Then we have the following.

(i) Suppose that the codomain of any arrow of $\mathcal{C}$ whose domain is equal to $A$ is isotropic. Then $\phi$ is co-angular. In particular, $\phi$ is a morphism of Frobenius type if and only if it is an isometric base-isomorphism.

(ii) Suppose that $\mathcal{C}$ is a Frobenioid. Then $\phi$ is a pull-back morphism if and only if it is an LB-invertible linear morphism (i.e., $a$ co-angular linear isometry).

(iii) Suppose that $\mathcal{C}$ is a Frobenioid. Then every LB-invertible pre-step is an isomorphism.

(iv) Suppose that $\mathcal{C}$ is a Frobenioid. Then a morphism $\phi$ of $\mathcal{C}$ is co-angular if and only if, in the factorization $\phi=\alpha \circ \beta \circ \gamma$ of Definition 1.3(iv)(a), the pre-step $\beta$ is co-angular. 
(v) Suppose that $\mathcal{C}$ is a Frobenioid. Then a morphism $\phi$ of $\mathcal{C}$ is LB-invertible if and only if it is of the form $\alpha \circ \beta$, where $\alpha$ is a pull-back morphism and $\beta$ is a morphism of Frobenius type.

Proof. Assertion (i) follows formally from the definitions of the terms 'isotropic', 'isometric pre-step', 'co-angular' and 'morphism of Frobenius type' (cf. Definition 1.2(i), (iii) and (iv)).

As for assertion (ii), if $\phi$ is a pull-back morphism, then it follows from Definition 1.3(iv)(b), that $\phi$ is an LB-invertible linear morphism. Now suppose that $\phi$ is LB-invertible and linear. Then by applying Remark 1.1.1 to the factorization of Definition 1.3(iv)(a), the fact that $\phi$ is a linear isometry implies that $\phi$ may be written in the form $\alpha \circ \beta$, where $\alpha$ is a pull-back morphism and $\beta$ is an isometric pre-step. On the other hand, since $\phi$ is co-angular, it follows that $\beta$ is an isomorphism, hence that $\phi$ is a pull-back morphism, as desired.

Assertion (iii) follows from either the uniqueness of the factorization of pre-steps of Definition 1.3(v)(b), or the essential uniqueness of morphisms of Frobenius type of a given Frobenius degree (cf. Definition 1.3(ii)).

Next, we consider assertion (iv). If $\beta$ is co-angular, then since $\alpha$ and $\gamma$ are co-angular (cf. assertion (ii); Definition 1.2(iii)), it follows from Definition 1.3(iii)(a), that $\phi$ is coangular. Conversely, if $\phi$ is co-angular and $\beta=\beta_{1} \circ \beta_{2} \circ \beta_{3}$, where $\beta_{2}$ is an isometric prestep, then by applying Remark 1.1.1, together with the fact that $\mathcal{D}$ is totally epimorphic (cf. the discussion of Section 0) to this factorization of $\beta$, we conclude that $\beta_{1}, \beta_{3}$ are pre-steps, hence that $\alpha \circ \beta_{1}$ is linear and that $\beta_{3} \circ \gamma$ is a base-isomorphism; thus, the co-angularity of $\phi=\left(\alpha \circ \beta_{1}\right) \circ \beta_{2} \circ\left(\beta_{3} \circ \gamma\right)$ implies that $\beta_{2}$ is an isomorphism, hence that $\beta$ is co-angular, as desired.

Finally, we consider assertion (v). If $\phi=\alpha \circ \beta$, where $\alpha$ is a pull-back morphism, and $\beta$ is a morphism of Frobenius type, then (since $\alpha, \beta$ are LB-invertible - cf. assertion (ii); Definition 1.2(iii)) it follows from Remark 1.1.1 that $\phi$ is isometric and from Definition 1.3(iii)(a), that $\phi$ is co-angular, hence $L B$-invertible. Now suppose that $\phi$ is $L B$ invertible, and that we have a factorization $\phi=\alpha \circ \beta \circ \gamma$, where $\alpha, \beta$ and $\gamma$ are as in Definition 1.3(iv)(a). By assertion (iv), $\beta$ is co-angular and by Remark 1.1.1, $\beta$ is isometric. Thus, $\beta$ is an LB-invertible pre-step, hence (cf. assertion (iii)) an isomorphism, as desired. This completes the proof of assertion (v).

Remark 1.4.1. We refer to the chart of types of morphisms in a Frobenioid given at the end of this paper for a summary of the properties of the base category projections, zero divisors and Frobenius degrees satisfied by various types of morphisms in a Frobenioid.

PROPOSITION 1.5. (Elementary Frobenioids are Frobenioids) Let $\Phi$ be a pre-divisorial monoid on a connected, totally epimorphic category $\mathcal{D}$. Then we have the following.

(i) $\mathbb{F}_{\Phi}$, equipped with the natural functor $\mathbb{F}_{\Phi} \rightarrow \mathbb{F}_{\Phi \text { char, }}$, is a Frobenioid of Aut-ample, Aut $^{\text {sub }}$-ample, End-ample, base-trivial, Frobenius-trivial, Frobenius-normalized and isotropic type.

(ii) There is a natural, functorial isomorphism

$$
\mathcal{O}^{\triangleright}(A) \stackrel{\sim}{\rightarrow} \Phi(A)
$$

( so $\left.\mathcal{O}^{\times}(A) \stackrel{\sim}{\rightarrow} \Phi(A)^{ \pm}\right)$for objects $A \in \mathrm{Ob}\left(\mathbb{F}_{\Phi}\right)$. 
(iii) If all of the monoids in the image of $\Phi$ are perfect (respectively, group-like), then $\mathbb{F}_{\Phi}$ is of perfect (respectively, group-like) type.

Proof. Since $\mathcal{D}$ is a connected, totally epimorphic category, the fact that $\mathbb{F}_{\Phi}$ is as well follows immediately from the definition of the morphisms of $\mathbb{F}_{\Phi}$ in Definition 1.1(iii), the fact that a pre-divisorial monoid is integral (cf. Definition 1.1(i)) and the injectivity condition of Definition 1.1(ii)(a). Thus, $\mathbb{F}_{\Phi}$ is a pre-Frobenioid. It is immediate from the definitions that assertion (ii) holds, and that all objects of $\mathbb{F}_{\Phi}$ are Aut-ample, Aut ${ }^{\text {sub }}$-ample, End-ample, basetrivial, Frobenius-trivial, Frobenius-normalized and isotropic. Also, one verifies immediately (cf. the definition of the category $\mathbb{F}_{\Phi}$ in Definition 1.1(iii)) that a morphism of $\mathbb{F}_{\Phi}$ is a pull-back morphism if and only if it is a linear isometry. The fact that $\mathbb{F}_{\Phi}$ satisfies the conditions of Definition 1.3 now follows immediately from the definition of the category $\mathbb{F}_{\Phi}$ in Definition 1.1(iii), together with assertion (ii) and the 'explicit description' of co-angular morphisms and morphisms of Frobenius type in Proposition 1.4(i) (which is applicable to all morphisms of $\mathbb{F}_{\Phi}$ since $\mathbb{F}_{\Phi}$ is of isotropic type). This completes the proof of assertion (i).

Assertion (iii) is immediate from the definitions and assertion (i).

One important technique for constructing new Frobenioids is given by the following result.

PROPOSITION 1.6. (Categorical fiber products) Let $\Phi$ be $a$ divisorial monoid on a connected, totally epimorphic category $\mathcal{D}$ and let $\mathcal{C} \rightarrow \mathbb{F}_{\Phi}$ be a Frobenioid. Let $\mathcal{D}^{\prime}$ be a connected, totally epimorphic category and let $\mathcal{D}^{\prime} \rightarrow \mathcal{D}$ be a functor that maps FSMmorphisms to FSM-morphisms. Denote by $\Phi^{\prime}: \mathcal{D}^{\prime} \rightarrow \mathfrak{M o n}$ the divisorial monoid obtained by restricting $\Phi$ to $\mathcal{D}^{\prime}$. Then we have the following.

(i) There is a natural equivalence of categories

$$
\mathbb{F}_{\Phi^{\prime}} \stackrel{\sim}{\rightarrow} \mathbb{F}_{\Phi} \times \mathcal{D} \mathcal{D}^{\prime}
$$

(where the latter category is the categorical fiber product of Section 0).

(ii) The categorical fiber product (cf. Section 0)

$$
\mathcal{C}^{\prime} \stackrel{\text { def }}{=} \mathcal{C} \times \mathcal{D} \mathcal{D}^{\prime}
$$

equipped with the functor $\mathcal{C}^{\prime} \rightarrow \mathbb{F}_{\Phi^{\prime}}$ (obtained by applying ' $(-) \times_{\mathcal{D}} \mathcal{D}^{\prime}$ ' to the functor $\left.\mathcal{C} \rightarrow \mathbb{F}_{\Phi}\right)$ is a Frobenioid.

(iii) A morphism of $\mathcal{C}^{\prime}$ is $a(n)$ isometry (respectively, morphism of a given Frobenius degree; co-angular morphism; LB-invertible morphism; pull-back morphism) if and only if its projection to $\mathcal{C}$ is.

(iv) A base-isomorphism of $\mathcal{C}^{\prime}$ is a morphism of Frobenius type (respectively, pre-step; step) if and only if its projection to $\mathcal{C}$ is. Moreover, the projection functor $\mathcal{C}^{\prime} \rightarrow \mathcal{C}$ determines $a$ bijection of monoids $\mathcal{O}^{\triangleright}\left(A^{\prime}\right) \stackrel{\sim}{\rightarrow} \mathcal{O}^{\triangleright}(A)$, for every $A^{\prime} \in \mathrm{Ob}\left(\mathcal{C}^{\prime}\right)$ that projects to $A \in \mathrm{Ob}(\mathcal{C})$.

(v) A object of $\mathcal{C}^{\prime}$ is Frobenius-trivial (respectively, quasi-Frobenius-trivial; sub-quasiFrobenius-trivial; metrically trivial; base-trivial; perfect; group-like; unit-trivial; Frobenius-normalized; isotropic; Frobenius-isotropic) if and only if it projects to such an object of $\mathcal{C}$. 
(vi) A object of $\mathcal{C}^{\prime}$ is Aut-ample (respectively, Aut ${ }^{\text {sub }}$-ample; End-ample) if it projects to such an object of $\mathcal{C}$.

Proof. Assertion (i) follows formally from the definitions. Next, observe that the fact that $\mathcal{D}^{\prime}$ is a totally epimorphic category implies immediately that $\mathcal{C}^{\prime}$ is as well; similarly, (in light of the various properties of the natural projection functor $\mathcal{C} \rightarrow \mathcal{D}$ assumed in Definition 1.3(i)(a), (b) and (c)) the fact that $\mathcal{D}^{\prime}$ is connected implies immediately that $\mathcal{C}^{\prime}$ is also connected. Thus, $\mathcal{C}^{\prime}$ (equipped with the functor $\mathcal{C}^{\prime} \rightarrow \mathbb{F}_{\Phi^{\prime}}$ obtained by applying ' $(-) \times{ }_{\mathcal{D}} \mathcal{D}^{\prime}$ ' to the functor $\mathcal{C} \rightarrow \mathbb{F}_{\Phi}$ ) is a pre-Frobenioid. Now assertion (vi) follows immediately from the definitions; one checks immediately that the equivalences of assertions (iii), (iv) and (v) hold. In light of these equivalences, the conditions of Definition 1.3 follow via a routine verification. Thus, $\mathcal{C}^{\prime}$ is a Frobenioid. This completes the proof of assertion (ii).

Proposition 1.7. (Composites of morphisms) Let $\Phi$ be a divisorial monoid on a connected, totally epimorphic category $\mathcal{D}$ and let $\mathcal{C} \rightarrow \mathbb{F}_{\Phi}$ be a Frobenioid. Then we have the following.

(i) The following classes of morphisms are closed under composition: isometries, baseisomorphisms, base-FSM-morphisms, pull-back morphisms, linear morphisms, presteps, co-angular morphisms, LB-invertible morphisms and morphisms of Frobenius type.

(ii) A morphism of $\mathcal{C}$ is a pull-back morphism if and only if it is minimal-adjoint to the base-isomorphisms of $\mathcal{C}$. A morphism of $\mathcal{C}$ is a base-isomorphism if and only if it is minimal-coadjoint to the pull-back morphisms of $\mathcal{C}$; alternatively, a morphism of $\mathcal{C}$ is a base-isomorphism if and only if it is may be written as a composite $\alpha \circ \beta$, where $\alpha$ is a pre-step, and $\beta$ is a morphism of Frobenius type.

(iii) A morphism of $\mathcal{C}$ is of Frobenius type if and only if it is minimal-coadjoint to the linear morphisms of $\mathcal{C}$. A morphism of $\mathcal{C}$ is linear if and only if it is minimal-adjoint to the morphisms of Frobenius type of $\mathcal{C}$; alternatively, a morphism of $\mathcal{C}$ is linear if and only if it is may be written as a composite $\alpha \circ \beta$, where $\alpha$ is a pull-back morphism and $\beta$ is a pre-step.

(iv) A pre-step of $\mathcal{C}$ is co-angular if and only if it is mid-adjoint (cf. Section 0) to the isometric pre-steps.

(v) If a composite morphism $\phi=\alpha \circ \beta$ of $\mathcal{C}$ is $a(n)$ isomorphism (respectively, baseisomorphism; linear morphism; pre-step; isometry; co-angular pre-step; co-angular linear morphism; pull-back morphism), then so are $\alpha$ and $\beta$. If, moreover, the domain of $\phi$ is isotropic, then a similar statement holds for morphisms of Frobenius type.

Proof. Assertion (i) follows immediately from the definitions for isometries, baseisomorphisms, base-FSM-morphisms, pull-back morphisms, linear morphisms and presteps, from Definition 1.3(iii)(a), for co-angular morphisms, hence also for LB-invertible morphisms and morphisms of Frobenius type. Next, the sufficiency of the various conditions given in assertions (ii) and (iii) follows immediately from (definitions and) the (existence of the) factorization of Definition 1.3(iv)(a). Moreover, in light of the existence of this factorization, the necessity of the various conditions given in assertions (ii) and (iii) follows immediately for pull-back morphisms and morphisms of Frobenius type from the essential uniqueness of this factorization (and the total epimorphicity of $\mathcal{C}$ ), for base-isomorphisms 
from the total epimorphicity of $\mathcal{D}$ and for linear morphisms from the well-known structure of the multiplicative monoid $\mathbb{N}_{\geq 1}$ and the essential uniqueness of morphisms of Frobenius type of a given Frobenius degree (cf. Definition 1.3(ii)).

In light of Remark 1.1.1, assertion (v) follows for isomorphisms (respectively, baseisomorphisms; linear morphisms; pre-steps; isometries) immediately from the fact that $\mathcal{C}$ is totally epimorphic (respectively, from the fact that $\mathcal{D}$ is totally epimorphic; from the wellknown structure of the multiplicative monoid $\mathbb{N}_{\geq 1}$; from assertion (v) for base-isomorphisms and linear morphisms; from the fact that the monoid $\Phi$ on $\mathcal{D}$ is sharp (cf. Definition 1.1(i)), together with the characteristic injectivity assumption of Definition 1.1(ii)(a)). Now assertion (iv) follows formally from (the definitions and) assertion (v) for pre-steps (cf. the argument applied in the proof of Proposition 1.4(iv)!); assertion (v) for co-angular pre-steps follows from assertion (v) for pre-steps and assertion (iv). To prove assertion (v) for co-angular linear morphisms, suppose that $\phi$ is co-angular and linear. Then observe that by assertion (v) for linear morphisms, $\alpha$ and $\beta$ are linear. Thus, by applying the factorization for linear morphisms of assertion (iii), together with the factorization of Definition 1.3(v)(c) (cf. also Proposition 1.4(ii); assertion (i) for co-angular linear morphisms), we may write $\alpha=\alpha_{1} \circ \alpha_{2}$ and $\beta=\beta_{1} \circ \beta_{2}, \alpha_{2} \circ \beta_{1}=\gamma_{1} \circ \gamma_{2}$, where $\alpha_{1}, \beta_{1}, \gamma_{1}$ are co-angular linear morphisms and $\alpha_{2}, \beta_{2}, \gamma_{2}$ are isometric pre-steps. Thus, $\phi=\left(\alpha_{1} \circ \gamma_{1}\right) \circ\left(\gamma_{2} \circ \beta_{2}\right)$, which (by the co-angularity of $\phi$ ) implies that $\gamma_{2} \circ \beta_{2}$ is an isomorphism, hence (by assertion (v) for isomorphisms) that $\beta_{2}$ and $\gamma_{2}$ are isomorphisms. Thus, by the co-angularity of $\alpha_{2} \circ \beta_{1}=\gamma_{1} \circ \gamma_{2}$, we conclude that $\alpha_{2}$ is an isomorphism. In particular, it follows that $\alpha$ and $\beta$ are co-angular linear morphisms, as desired. Now assertion (v) for pull-back morphisms follows from assertion (v) for co-angular linear isometries (cf. also Proposition 1.4(ii)). Finally, assertion (v) for morphisms of Frobenius type in $\mathcal{C}^{\text {istr }}$ (cf. Definition 1.3(vii)(b)) follows from assertion (v) for isometric base-isomorphisms, since morphisms of $\mathcal{C}^{\text {istr }}$ are always co-angular (cf. Proposition 1.4(i)). This completes the proof of assertion (v).

PROPOSITION 1.8. (Pre-steps) Let $\Phi$ be a divisorial monoid on a connected, totally epimorphic category $\mathcal{D}$ and let $\mathcal{C} \rightarrow \mathbb{F}_{\Phi}$ be a Frobenioid. Then we have the following.

(i) If the natural projection functor $\mathcal{C} \rightarrow \mathcal{D}$ is full, then every pre-step of $\mathcal{C}$ is a linear Endequivalence. If $\mathcal{D}$ is of Aut-type ( $c f$. Section 0 ), then every linear End-equivalence of $\mathcal{C}$ is a pre-step.

(ii) Suppose further that $\mathcal{C}$ is of metrically trivial and Aut-ample type. Then a morphism of $\mathcal{C}$ is a co-angular pre-step if and only if it is abstractly equivalent ( $c f$. Section 0 ) to a base-identity pre-step endomorphism of $\mathcal{C}$.

(iii) An object $A \in \mathrm{Ob}(\mathcal{C})$ is non-group-like if and only if there exists a co-angular step $A \rightarrow B$; alternatively, an object $A \in \mathrm{Ob}(\mathcal{C})$ is non-group-like if and only if there exists a co-angular step $B \rightarrow A$. Also, if $A, B \in \mathrm{Ob}(\mathcal{C})$ are base-isomorphic objects, then $A$ is group-like if and only if $B$ is.

Proof. First, we consider assertion (i). If $\phi \in \operatorname{Arr}(\mathcal{C})$ is a pre-step and the projection functor $\mathcal{C} \rightarrow \mathcal{D}$ is full, then the fact that it is a linear End-equivalence follows formally from the definition of a 'pre-step' (cf. Definition 1.2(iii)) and the fullness assumption on $\mathcal{C} \rightarrow \mathcal{D}$. On the other hand, if $\phi \in \operatorname{Arr}(\mathcal{C})$ is a linear End-equivalence and $\mathcal{D}$ is of Aut-type, then it follows formally that $\phi$ is a base-isomorphism, hence a pre-step, as desired. This completes the proof of assertion (i). 
Next, we consider assertion (ii). If $\phi \in \operatorname{Arr}(\mathcal{C})$ is a co-angular pre-step, then it follows formally from the assumption that $\mathcal{C}$ is of metrically trivial and Aut-ample type that $\phi$ is abstractly equivalent to a base-identity pre-step endomorphism of $\mathcal{C}$. On the other hand, if $\phi \in \operatorname{Arr}(\mathcal{C})$ is abstractly equivalent to a base-identity pre-step endomorphism of $\mathcal{C}$ (hence co-angular, by Definition 1.3(iii)(b)), then it follows formally that $\phi$ is a co-angular linear base-isomorphism, hence that $\phi$ is a co-angular pre-step, as desired. This completes the proof of assertion (ii). Finally, we observe that the various equivalences of assertion (iii) follow formally from the definitions and the equivalences of categories of Definition 1.3(iii)(d).

PROPOSITION 1.9. (Isotropic objects and isometries) Let $\Phi$ be a divisorial monoid on a connected, totally epimorphic category $\mathcal{D}$ and let $\mathcal{C} \rightarrow \mathbb{F}_{\Phi}$ be a Frobenioid. Write $\mathcal{C}^{\text {imtr-pre }} \subseteq$ $\mathcal{C}$ for the subcategory determined by the isometric pre-steps and

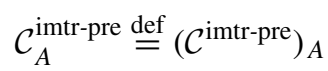

for $A \in \mathrm{Ob}(\mathcal{C})$. Then we have the following.

(i) Any base-isomorphism $\phi: A \rightarrow B$ of $\mathcal{C}$ admits a factorization

$$
\phi=\alpha \circ \beta
$$

where $\alpha$ is an isometric pre-step and $\beta$ is a co-angular base-isomorphism; this factorization is unique, up to replacing the pair $(\alpha, \beta)$ by a pair of the form ( $\alpha$ o $\gamma, \gamma^{-1} \circ \beta$ ), where $\gamma$ is an isomorphism of $\mathcal{C}$. Here, $\phi$ is isometric if and only if $\beta$ is a morphism of Frobenius type, $\phi$ is co-angular if and only if $\alpha$ is an isomorphism and $\phi$ is a pull-back morphism if and only if $\phi$ is an isomorphism.

(ii) Any base-isomorphism $\phi: A \rightarrow B$ of $\mathcal{C}$ induces a functor (well-defined up to isomorphism)

$$
\phi_{*}: \mathcal{C}_{A}^{\text {imtr-pre }} \rightarrow \mathcal{C}_{B}^{\text {imtr-pre }}
$$

that maps an isometric pre-step $C \rightarrow A$ to the isometric pre-step $D \rightarrow B$ appearing in the factorization $C \rightarrow D \rightarrow B$ of (i) applied to the composite of the given prestep $C \rightarrow A$ with $\phi: A \rightarrow B$. Moreover, if $\phi$ is a co-angular pre-step, then $\phi_{*}$ is an equivalence of categories. If $u \in \mathcal{O}^{\times}(A)$, then we shall denote by $u^{\text {imtr-pre }}$ the isomorphism class of the self-equivalence of the category $\mathcal{C}_{A}^{\text {imtr-pre }}$ induced by $u$ and by

$$
\mathcal{O}^{\times}(A)^{\text {imtr-pre }} \subseteq \mathcal{O}^{\times}(A)
$$

the subgroup of $v \in \mathcal{O}^{\times}(A)$ for which $v^{\text {imtr-pre }}$ is the identity.

(iii) Any pull-back morphism $\phi: A \rightarrow B$ of $\mathcal{C}$ induces a functor (well-defined up to isomorphism)

$$
\phi^{*}: \mathcal{C}_{B}^{\text {imtr-pre }} \rightarrow \mathcal{C}_{A}^{\text {imtr-pre }}
$$

that maps an isometric pre-step $\delta: D \rightarrow B$ to the unique (up to isomorphism) isometric pre-step $\gamma: C \rightarrow A$ that fits into a commutative diagram

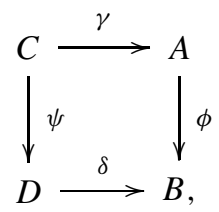


where $\psi$ is the pull-back morphism that arises by applying the equivalence of categories of Definition 1.3(i)(c) to the arrow $\operatorname{Base}(\delta)^{-1} \circ \operatorname{Base}(\phi)$ and $\gamma$ is the morphism that arises from the isomorphism of functors appearing in the definition of a 'pull-back morphism' (cf. Definition 1.2(ii)).

(iv) Let $\phi: A \rightarrow B$ be a co-angular linear morphism (e.g., a pull-back morphism $-c f$. Proposition 1.4(ii)). Then $A$ is isotropic if and only if $B$ is.

(v) $\mathcal{C}^{\text {istr }}$ (equipped with the restriction to $\mathcal{C}$ of the given functor $\mathcal{C} \rightarrow \mathbb{F}_{\Phi}$ ) is a Frobenioid. Moreover, the functor

$$
\mathcal{C} \rightarrow \mathcal{C}^{\text {istr }}
$$

that assigns to an object $A \in \mathrm{Ob}(\mathcal{C})$ with isotropic hull $A \rightarrow A^{\mathrm{istr}}$ the object $A^{\mathrm{istr}}$ and to a morphism of objects $A \rightarrow B$ with isotropic hulls $A \rightarrow A^{\text {istr }}, B \rightarrow B^{\text {istr }}$ the induced (i.e., by the definition of an 'isotropic hull'!) morphism $A^{\text {istr }} \rightarrow B^{\text {istr }}$ forms a left adjoint to the inclusion functor $\mathcal{C}^{\text {istr }} \hookrightarrow \mathcal{C}$, through which the functor $\mathcal{C} \rightarrow \mathbb{F}_{\Phi}$ factors. We shall refer to this functor as the isotropification functor. The restriction of the isotropification functor to $\mathcal{C}^{\text {istr }}$ is isomorphic to the identity functor. Finally, the isotropification functor preserves morphisms of Frobenius type, Frobenius degrees, pre-steps, pull-back morphisms, base-isomorphisms, base-FSM-morphisms, base-identity endomorphisms, Div-identity endomorphisms, isometries, co-angular morphisms and LB-invertible morphisms; moreover, all of these properties are compatible with the inclusion functor $\mathcal{C}^{\text {istr }} \hookrightarrow \mathcal{C}$ (in the sense that an arrow of $\mathcal{C}^{\text {istr }}$ satisfies one of these properties with respect to $\mathcal{C}^{\text {istr }}$ if and only if it does with respect to $\mathcal{C}$ ).

(vi) A morphism of $\mathcal{C}$ is an isotropic hull if and only if its codomain is isotropic, and, moreover, it is minimal-coadjoint to the morphisms with isotropic domain.

(vii) A morphism $A \rightarrow B$ of $\mathcal{C}$ is an isometric pre-step if and only if the composite of this morphism $A \rightarrow B$ with an isotropic hull $B \rightarrow C$ yields an isotropic hull $A \rightarrow C$.

Proof. Since pull-backs which are base-isomorphisms are easily verified to be isomorphisms (cf. Remark 1.2.1), assertion (i) follows immediately from the (essentially) unique factorization of Definition 1.3(iv)(a), the (essentially) unique factorization of pre-steps of Definition $1.3(\mathrm{v})(\mathrm{b})$, the fact that co-angular morphisms are closed under composition (cf. Proposition 1.7(i)), the definition of 'co-angular' (cf. Definition 1.2(iii)), the fact that $\mathcal{C}$ is totally epimorphic, the essential uniqueness of morphisms of Frobenius type of a given Frobenius degree (cf. Definition 1.3(ii)) and Remark 1.1.1.

Next, we consider assertion (ii). The existence of the functor $\phi_{*}$ follows formally from the existence of the (essentially) unique factorization of assertion (i). Now suppose that $\phi$ is a co-angular pre-step. Then for any isometric pre-step $\beta: D \rightarrow B$, there exists a co-angular pre-step $\psi: C \rightarrow D$ such that

$$
\left.(\Phi(\beta \circ \psi))^{-1}(\operatorname{Div}(\psi))\right)=(\Phi(\phi))^{-1}(\operatorname{Div}(\phi))
$$

(cf. the second equivalence of categories of Definition 1.3(iii)(d)). Thus, by applying the factorization of Definition 1.3(v)(c), it follows that we may write $\beta \circ \psi=\phi^{\prime} \circ \alpha^{\prime}$, where $\alpha^{\prime}: D \rightarrow A^{\prime}$ is an isometric pre-step and $\phi^{\prime}: A^{\prime} \rightarrow B$ is a co-angular pre-step. On the other hand, since $\operatorname{Div}(\beta \circ \psi)=\operatorname{Div}\left(\phi^{\prime} \circ \alpha^{\prime}\right)$ and $\beta, \alpha^{\prime}$ are isometric, it follows that

$$
(\Phi(\phi))^{-1}(\operatorname{Div}(\phi))=\left(\Phi\left(\phi^{\prime}\right)\right)^{-1}\left(\operatorname{Div}\left(\phi^{\prime}\right)\right),
$$


hence (by the second equivalence of categories of Definition 1.3(iii)(d)) that there exists an isomorphism $\gamma: A^{\prime} \stackrel{\sim}{\rightarrow} A$ such that $\phi \circ \gamma=\phi^{\prime}$. Thus, if we take $\alpha \stackrel{\text { def }}{=} \gamma \circ \alpha^{\prime}$, then $\beta \circ \psi=$ $\phi \circ \alpha$ - that is to say, $\phi_{*}$ is essentially surjective. Moreover, (by possibly replacing $\phi$ by $\psi$ ) this argument (i.e., the construction, given $\beta, \phi$, of $\alpha, \psi$ such that $\beta \circ \psi=\phi \circ \alpha$ ) also implies that $\phi_{*}$ is full. Finally, since every pre-step is a monomorphism (cf. Definition 1.3(v)(a)), it follows immediately that $\phi_{*}$ is faithful. This completes the proof of assertion (ii).

Assertion (iii) follows formally from the definitions, together with the fact that pull-back morphisms are linear isometries (cf. Proposition 1.4(ii)), which implies (cf. Remark 1.1.1) that $\gamma$ is an isometric pre-step.

Next, we consider assertion (iv). Let $\phi: A \rightarrow B$ be a co-angular linear morphism. If $A$ is isotropic, then so is $B$ by Definition 1.3(vii)(b). Now suppose that $B$ is isotropic. Thus, by the definition of an isotropic hull, it follows from the existence of isotropic hulls (cf. Definition 1.3(vii)(a)) that there exists a factorization $\phi=\beta \circ \alpha$, where $\alpha: A \rightarrow A^{\prime}$ is an isotropic hull (hence an isometric pre-step - cf. Definition 1.2(iv)) and $\beta: A^{\prime} \rightarrow B$ is linear (cf. Remark 1.1.1). Thus, by the definition of 'co-angular' (cf. Definition 1.2(iii)), we conclude that $\alpha$ is an isomorphism, as desired. This completes the proof of assertion (iv).

Next, we consider assertion (v). By applying the definition of an isotropic hull (cf. Definition 1.2(iv)), it follows immediately (from the fact that $\mathcal{C}$ is connected and totally epimorphic) that $\mathcal{C}^{\text {istr }}$ is connected and totally epimorphic. Thus, $\mathcal{C}^{\text {istr }}$ is a pre-Frobenioid. It is immediate from the definition of an isotropic hull that the isotropification functor is left adjoint to the inclusion functor $\mathcal{C}^{\text {istr }} \hookrightarrow \mathcal{C}$, that the functor $\mathcal{C} \rightarrow \mathbb{F}_{\Phi}$ factors through the isotropification functor (cf. Remark 1.1.1), that the restriction of the isotropification functor to $\mathcal{C}^{\text {istr }}$ is isomorphic to the identity functor and (cf. Remark 1.1.1) that the isotropification functor preserves Frobenius degrees, pre-steps, base-isomorphisms, baseFSM-morphisms, base-identity endomorphisms, Div-identity endomorphisms, isometries and co-angular morphisms (cf. Proposition 1.4(i)), hence also LB-invertible morphisms and morphisms of Frobenius type in a fashion that is compatible (cf. the statement of assertion (v)) with the inclusion $\mathcal{C}^{\text {istr }} \hookrightarrow \mathcal{C}$. Since pull-back morphisms are co-angular linear isometries (cf. Proposition 1.4(ii)), it follows immediately (in light of what we have shown so far) from Proposition 1.4(ii), that the isotropification functor maps pull-back morphisms to morphisms which are pull-back morphisms relative to $\mathcal{C}$, hence a fortiori, pull-back morphisms relative to $\mathcal{C}^{\text {istr. }}$. Finally, in light of Proposition 1.4(i), assertion (iv) (cf. also Definition 1.3(vii)(b)), it follows immediately (from the fact that $\mathcal{C}$ is a Frobenioid!) that the pre-Frobenioid $\mathcal{C}^{\text {istr }}$ satisfies the various conditions of Definition 1.3, hence that $\mathcal{C}^{\text {istr }}$ is a Frobenioid, as desired. This completes the proof of assertion (v).

Finally, we observe that the necessity and sufficiency of the condition of assertion (vi) follow immediately from the definition of an isotropic hull (cf. Definition 1.2(iv)), the existence of isotropic hulls (cf. Definition 1.3(vii)(a)) and the total epimorphicity of $\mathcal{C}$. The necessity and sufficiency of the condition of assertion (vii) follow immediately from the existence of isotropic hulls (cf. Definition 1.3(vii)(a)), the fact that isometric pre-steps between isotropic objects are isomorphisms (cf. Definition 1.3(vii)(b) and Proposition 1.4(i) and (iii)) and the following observation (which follows immediately from Proposition 1.7(i) and (v)): given morphisms $\alpha, \beta, \gamma$ of $\mathcal{C}$ such that $\gamma=\alpha \circ \beta$, if any two of the three morphisms $\alpha, \beta, \gamma$ is an isometric pre-step, then the same is true of the remaining morphism. 
Proposition 1.10. (Morphisms of Frobenius type) Let $\Phi$ be a divisorial monoid on a connected, totally epimorphic category $\mathcal{D}$ and let $\mathcal{C} \rightarrow \mathbb{F}_{\Phi}$ be a Frobenioid. Then we have the following.

(i) Let $\phi: A \rightarrow B$ be an arbitrary morphism of $\mathcal{C}$. Suppose that $\alpha: A \rightarrow A^{\prime}$ and $\beta: B \rightarrow$ $B^{\prime}$ are morphisms of Frobenius type, of Frobenius degree $d \in \mathbb{N}_{\geq 1}$. Then there exists a unique morphism $\phi^{\prime}: A^{\prime} \rightarrow B^{\prime}$ such that the following diagram commutes:

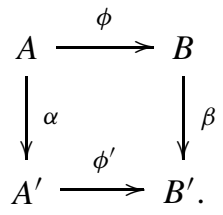

In this situation, $\operatorname{deg}_{\mathrm{Fr}}(\phi)=\operatorname{deg}_{\mathrm{Fr}}\left(\phi^{\prime}\right)$ and $\operatorname{Div}\left(\phi^{\prime}\right)=d \cdot \alpha_{*}(\operatorname{Div}(\phi))$ (where we write $\alpha_{*}: \Phi(A) \stackrel{\sim}{\rightarrow} \Phi\left(A^{\prime}\right)$ for the bijection induced by applying the functor $\Phi$ to the base-isomorphism $\alpha$ ). Finally, if $\phi$ is a morphism of Frobenius type (respectively, pre-step; pull-back morphism; co-angular morphism; base-isomorphism; isometry; LB-invertible morphism), then the same is true of $\phi^{\prime}$.

(ii) Any composite morphism $\beta \circ \alpha$ of $\mathcal{C}$, where $\alpha$ is a pre-step and $\beta$ is of Frobenius type, may be written as a composite

$$
\alpha^{\prime} \circ \beta^{\prime}=\beta \circ \alpha,
$$

where $\alpha^{\prime}$ is a pre-step and $\beta^{\prime}$ is of Frobenius type such that

$$
\operatorname{deg}_{\mathrm{Fr}}(\beta)=\operatorname{deg}_{\mathrm{Fr}}\left(\beta^{\prime}\right), \quad \operatorname{Div}\left(\alpha^{\prime}\right)=\operatorname{deg}_{\mathrm{Fr}}(\beta) \cdot \beta_{*}^{\prime}(\operatorname{Div}(\alpha))
$$

(where we write $\beta_{*}^{\prime}$ for the bijection induced by applying the functor $\Phi$ to the baseisomorphism $\beta^{\prime}$ ).

(iii) Suppose that $\mathcal{C}$ is of perfect type. Then the monoids in the image of $\Phi$ are perfect. If, moreover, $\mathcal{C}$ is of isotropic and Frobenius-normalized type, then the monoids $\mathcal{O}^{\triangleright}(A)$ and $\mathcal{O}^{\times}(A)$ are perfect.

(iv) A morphism of Frobenius type with isotropic domain is a prime-Frobenius morphism if and only if it is irreducible ( $c f$. Section 0). In particular, if $A \in \mathrm{Ob}(\mathcal{C})$ is isotropic, then there exist infinitely many isomorphism classes of objects of ${ }_{A} \mathcal{C}$ that arise from irreducible arrows with domain A.

(v) A morphism of $\mathcal{C}$ is a morphism of Frobenius type if and only if it is a composite of prime-Frobenius morphisms.

(vi) The Frobenioid $\mathcal{C}^{\text {istr }}$ is of sub-quasi-Frobenius-trivial type. Moreover, every group-like object $A \in \mathrm{Ob}\left(\mathcal{C}^{\text {istr }}\right)$ is Frobenius-trivial.

Proof. First, we consider assertion (i). Observe that uniqueness follows from the fact that $\mathcal{C}$ is totally epimorphic. Now it suffices to prove the existence of $\phi^{\prime}$ as desired, first in the case where $\phi$ is a morphism of Frobenius type, then in the case where $\phi$ is a pre-step and finally in the case where $\phi$ is a pull-back morphism (cf. the factorization of Definition 1.3(iv)(a)). In the first case, since morphisms of Frobenius type are closed under composition, with multiplying Frobenius degrees (cf. Proposition 1.7(i) and Remark 1.1.1), the existence of a morphism of Frobenius type $\phi^{\prime}$ as desired follows immediately from the existence and 
(essential) uniqueness of morphisms of Frobenius type of a given Frobenius degree (cf. Definition 1.3(ii)). In the case where $\phi$ is a pre-step, the existence of a pre-step $\phi^{\prime}$ (which, moreover, is co-angular if $\phi$ is) as desired follows immediately from the factorization of Definition 1.3(iv)(a) (cf. also Proposition 1.4(iv)), together with the (essential) uniqueness of morphisms of Frobenius type of a given Frobenius degree (cf. Definition 1.3(ii)) and the fact that co-angular morphisms are closed under composition (cf. Proposition 1.7(i)). In a similar vein, since pull-back morphisms are LB-invertible (cf. Proposition 1.4(ii)) and LB-invertible morphisms are closed under composition (cf. Proposition 1.7, (i)), the existence of a pullback morphism $\phi^{\prime}$ in the case where $\phi$ is a pull-back morphism follows immediately from the factorization of Proposition 1.4(v), together with the (essential) uniqueness of morphisms of Frobenius type of a given Frobenius degree (cf. Definition 1.3(ii)). The portion of assertion (i) concerning ' $\operatorname{deg}_{\mathrm{Fr}}(-)$ ' and 'Div(-)' then follows immediately from Remark 1.1.1. Finally, in light of what we have done so far, the fact that 'if $\phi$ is a(n) co-angular morphism (respectively, base-isomorphism; isometry; LB-invertible morphism), then the same is true of $\phi^{\prime}$ ' follows immediately from the definitions, Remark 1.1.1, the factorization of co-angular morphisms given in Proposition 1.4(iv) and the fact that co-angular morphisms are closed under composition (cf. Proposition 1.7(i)). This completes the proof of assertion (i). Now (in light of the existence of morphisms of Frobenius type of a given Frobenius degree - cf. Definition 1.3(ii)) assertion (ii) follows formally from assertion (i).

Next, we consider assertion (iii). In light of the existence of morphisms of Frobenius type of a given Frobenius degree (cf. Definition 1.3(ii)) and the equivalences of categories of Definition 1.3(iii)(d), the fact that $\Phi(A)$ is perfect follows immediately (cf. Remark 1.1.1) from the fact that $A$ is perfect (cf. Definition 1.2(iv)). Now suppose further that $\mathcal{C}$ is of isotropic (so all morphisms of $\mathcal{C}$ are co-angular - cf. Proposition 1.4(i)) and Frobeniusnormalized type. Then by the existence of Frobenius-trivial objects (cf. Definition 1.3(i)(a) and (b) and the isomorphism of Definition 1.3(iii)(c)), we may assume that $A$ is Frobenius-trivial. Now the fact that the monoids $\mathcal{O}^{\triangleright}(A)$ and $\mathcal{O}^{\times}(A)$ are perfect follows immediately from the fact that $A$ is perfect (cf. Definition 1.2(iv), applied to the baseidentity endomorphisms of Frobenius type of the Frobenius-trivial object $A$ ) and Frobeniusnormalized. This completes the proof of assertion (iii).

Next, we observe that assertion (iv) follows immediately from Proposition 1.7(v) and the well-known structure of the multiplicative monoid $\mathbb{N}_{\geq 1}$ (cf. also Definition 1.3(ii)), and that assertion (v) follows immediately from Proposition 1.7(i) and Definition 1.3(ii).

Finally, we consider assertion (vi). Let $A \in \mathrm{Ob}\left(\mathcal{C}^{\text {istr }}\right)$. Then by Definition 1.3(i)(a) and (b) (applied to the Frobenioid $\mathcal{C}^{\text {istr }}-\mathrm{cf}$. Proposition 1.9(v)), there exist co-angular (cf. Proposition 1.4(i)) pre-steps $\alpha: B \rightarrow A$ and $\gamma: B \rightarrow C$, where $C$ is Frobenius-trivial. Thus, for $d \in \mathbb{N}_{\geq 1}$, there exists a base-identity endomorphism of Frobenius type $\phi_{C} \in \operatorname{End}_{\mathcal{C}}(C)$ such that $\operatorname{deg}_{\mathrm{Fr}}\left(\phi_{C}\right)=d$ and by assertion (ii) (cf. also Proposition 1.4(i)) we may write $\phi_{C} \circ \gamma=\gamma^{\prime} \circ \psi$, where $\psi: B \rightarrow B^{\prime}$ is a morphism of Frobenius type, and $\gamma^{\prime}: B^{\prime} \rightarrow C$ is a co-angular pre-step. Moreover, the portion of assertion (ii) concerning the relationship between $\operatorname{Div}(\gamma)$ and $\operatorname{Div}\left(\gamma^{\prime}\right)$ implies, in light of the second equivalence of categories of Definition 1.3(iii)(d), that $\gamma^{\prime}$ factors through $\gamma$, i.e., there exists a co-angular pre-step $\beta: B^{\prime} \rightarrow B$ such that $\gamma \circ \beta=\gamma^{\prime}$. Thus, if we set $\phi_{B} \stackrel{\text { def }}{=} \beta \circ \psi \in \operatorname{End}_{\mathcal{C}}(B)$, then $\gamma \circ \phi_{B}=$ $\phi_{C} \circ \gamma$. Moreover, since $\phi_{C}$ is a base-identity endomorphism of Frobenius degree $d$, and $\gamma$ is a pre-step, it follows (cf. Remark 1.1.1) that $\phi_{B}$ is also a base-identity endomorphism 
of Frobenius degree $d$. Thus, we conclude that $B$ is quasi-Frobenius-trivial, hence that $A$ is sub-quasi-Frobenius-trivial, as desired. If, moreover, $A$ is group-like, then (since $\mathcal{C}^{\text {istr }}$ is a Frobenioid - cf. Proposition 1.9(v)) it follows from Definition 1.3(i)(a) and (b), that there exist (co-angular - cf. Proposition 1.4(i)) pre-steps $A^{\prime} \rightarrow A, A^{\prime} \rightarrow A^{\prime \prime}$, where $A^{\prime \prime}$ is Frobenius-trivial. But by Proposition 1.4(iii), these pre-steps are isomorphisms, so $A$ is Frobenius-trivial, as desired. This completes the proof of assertion (vi).

PROPOSITION 1.11. (Pull-back and linear morphisms) Let $\Phi$ be a divisorial monoid on a connected, totally epimorphic category $\mathcal{D}$ and let $\mathcal{C} \rightarrow \mathbb{F}_{\Phi}$ be a Frobenioid. Then we have the following.

(i) Suppose further that $\mathcal{C}$ is of Aut-ample and base-trivial type. Then the natural projection functor $\mathcal{C}^{\mathrm{pl}-\mathrm{bk}} \rightarrow \mathcal{D}$ is full.

(ii) Suppose further that $\mathcal{C}$ is of unit-trivial type. Then the natural projection functor $\mathcal{C}^{\mathrm{pl}-\mathrm{bk}} \rightarrow \mathcal{D}$ is faithful.

(iii) Let $\phi: B \rightarrow A$ be a pull-back morphism that projects to a morphism $\phi_{\mathcal{D}} \stackrel{\text { def }}{=} \operatorname{Base}(\phi)$ : $B_{\mathcal{D}} \rightarrow A_{\mathcal{D}}$ of $\mathcal{D}$. Then given any $\alpha \in \operatorname{End}_{\mathcal{C}}(A)$ and $\beta_{\mathcal{D}} \in \operatorname{End}_{\mathcal{D}}\left(B_{\mathcal{D}}\right)$ such that $\operatorname{Base}(\alpha) \circ \phi_{\mathcal{D}}=\phi_{\mathcal{D}} \circ \beta_{\mathcal{D}}$, there exists a unique $\beta \in \operatorname{End}_{\mathcal{C}}(B)$ such that $\operatorname{Base}(\beta)=\beta_{\mathcal{D}}$, $\alpha \circ \phi=\beta \circ \phi$.

(iv) Every co-angular linear morphism $\phi: B \rightarrow$ A determines an injection of monoids

$$
\mathcal{O}^{\triangleright}(A) \hookrightarrow \mathcal{O}^{\triangleright}(B)
$$

which is uniquely determined by the condition that $\mathcal{O}^{\triangleright}(A) \ni \alpha \mapsto \beta \in \mathcal{O}^{\triangleright}(B)$ implies $\alpha \circ \phi=\phi \circ \beta$.

(v) The equivalences of categories of Definition 1.3(iii)(d) are 'functorial' in the following sense: if $\phi: A \rightarrow B$ is an arbitrary morphism of $\mathcal{C}^{\text {lin }}, \alpha: C \rightarrow A$ and $\beta: D \rightarrow B$ (respectively, $\alpha: A \rightarrow C$ and $\beta: B \rightarrow D$ ) are co-angular pre-steps such that $\left(\alpha^{*}\right)^{-1}(\operatorname{Div}(\alpha))=\phi^{*}\left\{\left(\beta^{*}\right)^{-1}(\operatorname{Div}(\beta))\right\}\left(\right.$ respectively, $\left.\operatorname{Div}(\alpha)=\phi^{*}(\operatorname{Div}(\beta))\right)$, then there exists a unique morphism $\psi: C \rightarrow D$ in $\mathcal{C}^{\text {lin }}$ such that $\beta \circ \psi=\phi \circ \alpha$ (respectively, $\psi \circ \alpha=\beta \circ \phi$ ). Moreover, $\phi$ is a pull-back morphism if and only if $\psi$ is.

(vi) A pull-back morphism $\phi \in \operatorname{Arr}(\mathcal{C})$ is an FSM-morphism (respectively, fiberwisesurjective morphism; monomorphism; irreducible morphism) if and only if $\operatorname{Base}(\phi) \in$ $\operatorname{Arr}(\mathcal{D})$ is.

(vii) Let $\phi: A \rightarrow B$ be a co-angular pre-step and let $\epsilon: C \rightarrow B$ be a morphism. Then there exists a co-angular pre-step $\gamma: D \rightarrow C$ and a morphism $\alpha: D \rightarrow A$ such that $\epsilon \circ \gamma=\phi \circ \alpha$. In particular, every co-angular pre-step of $\mathcal{C}$ is an FSM-morphism.

Proof. First, we consider assertion (i). Let $A, B \in \mathrm{Ob}(\mathcal{C}), A_{\mathcal{D}} \stackrel{\text { def }}{=} \operatorname{Base}(A), B \stackrel{\text { def }}{=} \operatorname{Base}(B)$ and let $\phi_{\mathcal{D}}: A_{\mathcal{D}} \rightarrow B_{\mathcal{D}}$ be a morphism in $\mathcal{D}$. By the equivalence of categories of Definition 1.3(i)(c), it follows that there exists a pull-back morphism $\psi: C \rightarrow B$ of $\mathcal{C}$ such that $\psi_{\mathcal{D}} \stackrel{\text { def }}{=} \operatorname{Base}(\psi): C_{\mathcal{D}} \rightarrow B_{\mathcal{D}}$ of $\mathcal{D}$ defines an object of $\mathcal{D}_{B_{\mathcal{D}}}$ that is isomorphic to the object defined by $\phi_{\mathcal{D}}$. In particular, $C_{\mathcal{D}}$ is isomorphic to $A_{\mathcal{D}}$. Since $\mathcal{C}$ is of base-trivial type, it thus follows that $A$ and $C$ are isomorphic, so we may assume that $A=C$. Thus, $\psi$ projects to a morphism $\psi_{\mathcal{D}}: A_{\mathcal{D}} \rightarrow B_{\mathcal{D}}$ of $\mathcal{D}$ such that $\phi_{\mathcal{D}}=\psi_{\mathcal{D}} \circ \delta$, for some $\delta \in \operatorname{Aut}_{\mathcal{D}}\left(A_{\mathcal{D}}\right)$. Since $\mathcal{C}$ is of Aut-ample type, it thus follows that $\delta$ lifts to a $\gamma \in \operatorname{Aut}_{\mathcal{C}}(A)$. Thus, taking $\psi \circ \gamma: A \rightarrow B$ yields a morphism of $\mathcal{C}$ that projects to $\phi_{\mathcal{D}}$. This completes the proof of assertion (i). 
Next, we consider assertion (ii). Let $A, B \in \mathrm{Ob}(\mathcal{C}), A_{\mathcal{D}} \stackrel{\text { def }}{=} \operatorname{Base}(A), B_{\mathcal{D}} \stackrel{\text { def }}{=} \operatorname{Base}(B)$ and let $\phi, \psi: A \rightarrow B$ be pull-back morphisms of $\mathcal{C}$ that project to the same morphism $A_{\mathcal{D}} \rightarrow B_{\mathcal{D}}$ of $\mathcal{D}$. By the definition of a 'pull-back morphism' (cf. Definition 1.2(ii)), it thus follows formally that there exist base-identity endomorphisms $\alpha, \beta \in \operatorname{End}_{\mathcal{C}}(A)$ such that $\psi=\phi \circ \alpha$ and $\phi=\psi \circ \beta$. In particular, we obtain that $\psi=\psi \circ \beta \circ \alpha$ and $\phi=\phi \circ \alpha \circ \beta$, hence (again by Definition 1.2(ii)) that $\alpha \circ \beta$ and $\beta \circ \alpha$ are both equal to the identity endomorphism of $A$, i.e., that $\alpha, \beta \in \operatorname{Aut}_{\mathcal{C}}(A)$. But this implies that $\alpha, \beta \in \mathcal{O}^{\times}(A)=\{1\}$, so $\phi=\psi$, as desired. This completes the proof of assertion (ii).

Next, we consider assertion (iii). The existence and uniqueness of $\beta$ as asserted follows immediately from the isomorphism of functors appearing in the definition of a 'pull-back morphism' (cf. Definition 1.2(ii)). This completes the proof of assertion (iii).

Now since a co-angular linear morphism factors as the composite of a pull-back morphism with a co-angular pre-step (cf. Propositions 1.4(iv) and 1.7(iii)), the existence of the map ' $\hookrightarrow$ ' of assertion (iv) follows immediately (cf. Proposition 1.7(iii)) from assertion (iii) and Definition 1.3(iii)(c). The asserted injectivity of this map follows from the total epimorphicity of $\mathcal{C}$; the fact that this map is uniquely determined by the condition given in assertion (iii) follows from the fact that pre-steps are monomorphisms (cf. Definition 1.3(v)(a)) and the definition of a 'pull-back morphism' in Definition 1.2(ii).

Next, we consider assertion (v). First, we observe that the uniqueness of $\psi$ follows from the fact that $\beta$ is a monomorphism (cf. Definition 1.3(v)(a)) in the non-resp'd case and from the total epimorphicity of $\mathcal{C}$ applied to $\alpha$ in the resp'd case. When $\phi$ is a pull-back morphism (hence co-angular and linear - cf. Proposition 1.4(ii)), the existence of a pull-back morphism $\psi$ as desired follows immediately by applying the equivalence of categories induced by the projection functor in Definition 1.3(i)(c), the definition of a 'pull-back morphism' in Definition 1.2(ii), Proposition 1.7(i)(v) (applied to co-angular linear morphisms) and the equivalences of categories of Definition 1.3(iii)(d). When $\phi$ is an isometric pre-step, the existence of an isometric pre-step $\psi$ as desired follows immediately from the equivalence of categories of Proposition 1.9(ii) (in the 'case of a co-angular pre-step'). When $\phi$ is a co-angular pre-step, the existence of a co-angular pre-step $\psi$ as desired follows formally from the equivalences of categories of Definition 1.3(iii)(d). In light of the factorizations of Definition 1.3(v)(b) and (c) and Proposition 1.7(iii), this completes the proof of assertion (v).

Next, we observe that assertion (vi) follows formally from the isomorphism of functors appearing in the definition of a 'pull-back morphism' (cf. Definition 1.2(ii)), together with the equivalence of categories induced by the projection functor in Definition 1.3(i)(c) (cf. also Proposition 1.7(v), for pull-back morphisms).

Finally, we consider assertion (vii). By applying the factorizations of Definition 1.3(iv)(a) and Definition 1.3(v)(b), it follows immediately that we may assume without loss of generality (from the point of view of showing the existence of $\gamma$ and $\alpha$ with the desired properties) that $\epsilon$ is a pull-back morphism, an isometric pre-step, a co-angular pre-step, or a morphism of Frobenius type. If $\epsilon$ is a pull-back morphism, then it follows immediately (by 'pulling back the zero divisor of $\phi$ via $\epsilon$ ' - cf. assertion (v)) that there exist a pullback morphism $\alpha: D \rightarrow A$ and a co-angular pre-step $\gamma: D \rightarrow C$ such that $\epsilon \circ \gamma=\phi \circ \alpha$. Next, observe that if $\epsilon$ is an isometric pre-step, then the existence of $\gamma$ and $\alpha$ with the desired properties follows formally from the equivalence of categories of Proposition 1.9(ii) (induced by $\phi$ ). Next, observe that if $\epsilon$ is a co-angular pre-step, then it follows immediately 
from the second equivalence of categories of Definition 1.3(iii)(d), that there exist co-angular pre-steps $\alpha: D \rightarrow A$ and $\gamma: D \rightarrow C$ such that $\epsilon \circ \gamma=\phi \circ \alpha$. Finally, we consider the case where $\epsilon$ is a morphism of Frobenius type. By applying the second equivalence of categories of Definition 1.3(iii)(d), it follows that we may assume (by replacing $\phi$ by the composite of $\phi$ with an appropriate pre-step $A^{\prime} \rightarrow A$ ) that $\operatorname{Div}(\phi)=\operatorname{deg}_{\mathrm{Fr}}(\epsilon) \cdot x$, for some $x \in \Phi(A)$. Thus (by applying again the second equivalence of categories of Definition 1.3(iii)(d)), it follows that there exist a morphism of Frobenius type $\alpha: D \rightarrow A$ and a co-angular pre-step $\gamma: D \rightarrow C$ such that $\epsilon \circ \gamma=\phi \circ \alpha$ (cf. also Proposition 1.10(i)). This completes the proof of the existence of $\gamma$ and $\alpha$ with the desired properties. It thus follows formally that every co-angular pre-step of $\mathcal{C}$ is fiberwise surjective. On the other hand, by Definition 1.3(v)(a), every pre-step is a monomorphism. Thus, we conclude that every co-angular pre-step of $\mathcal{C}$ is an FSM-morphism. This completes the proof of assertion (vii).

Remark 1.11.1. Observe that in the situation of Proposition 1.11(iii), if $\alpha$ is a morphism of Frobenius type and $\beta_{\mathcal{D}}$ is an isomorphism, then $\beta$ is a morphism of Frobenius type. (Indeed, then $\beta$ is co-angular by Definition 1.3(iii)(b) and isometric by Remark 1.1.1.) In particular, it follows (cf. Remark 1.2.1) that (at least in the case of Frobenioids) 'Frobenius-trivial' implies 'universally Div-Frobenius-trivial'.

PROPOSITION 1.12. (Endomorphisms) Let $\Phi$ be a divisorial monoid on a connected, totally epimorphic category $\mathcal{D}$ let $\mathcal{C} \rightarrow \mathbb{F}_{\Phi}$ be a Frobenioid, $A \in \operatorname{Ob}(\mathcal{C})$ and $A_{\mathcal{D}} \stackrel{\text { def }}{=} \operatorname{Base}(A) \in$ $\mathrm{Ob}(\mathcal{D})$. Then we have the following.

(i) We have natural exact sequences of monoids

$$
\begin{gathered}
1 \rightarrow \mathcal{O}^{\times}(A) \rightarrow \operatorname{Aut}_{\mathcal{C}}(A) \rightarrow \operatorname{Aut}_{\mathcal{D}}\left(A_{\mathcal{D}}\right), \\
1 \rightarrow \mathcal{O}^{\triangleright}(A) \rightarrow \operatorname{End}_{\mathcal{C}}(A) \rightarrow \mathbb{N}_{\geq 1} \times \operatorname{End}_{\mathcal{D}}\left(A_{\mathcal{D}}\right),
\end{gathered}
$$

where the second arrow in each sequence is the natural inclusion, the third arrow of the first sequence is determined by the natural projection functor to $\mathcal{D}$, the third arrow of the second sequence is determined by the Frobenius degree and the natural projection functor to $\mathcal{D}$. If, moreover, $A$ is Aut-ample (respectively, End-ample; quasi-Frobeniustrivial), then the map $\operatorname{Aut}_{\mathcal{C}}(A) \rightarrow \operatorname{Aut}_{\mathcal{D}}\left(A_{\mathcal{D}}\right)$ (respectively, $\operatorname{End}_{\mathcal{C}}(A) \rightarrow \operatorname{End}_{\mathcal{D}}\left(A_{\mathcal{D}}\right)$; $\left.\operatorname{End}_{\mathcal{C}}(A) \rightarrow \mathbb{N}_{\geq 1}\right)$ is surjective.

(ii) An endomorphism of $A$ is a sub-automorphism (cf. Section 0) if and only if it is an isometric linear endomorphism that projects to a sub-automorphism of $\mathcal{D}$.

(iii) A sub-automorphism of $A$ is an automorphism if and only if it is a base-isomorphism.

(iv) Suppose that A is Aut ${ }^{\text {sub }}$-ample. Then A is Aut-saturated (cf. Section 0) if and only if $A_{\mathcal{D}}$ is.

Proof. Assertion (i) is immediate from the definitions. The necessity of the conditions of assertion (ii) and (iii) is immediate from Remark 1.1.1. To prove the sufficiency of the conditions of assertion (ii) and (iii), it suffices, in light of the equivalence of categories (involving pull-back morphisms) of Definition 1.3(i)(c) (cf. also Proposition 1.11(iii)), and the fact that endomorphisms are always co-angular (cf. Definition 1.3(iii)(b)), to observe that any LB-invertible linear base-isomorphism (i.e., LB-invertible pre-step) is, in fact, an isomorphism (cf. Proposition 1.4(iii)). Now assertion (iv) follows formally from assertions (ii) and (iii) and the definitions. 
PROPOSITION 1.13. (Rigidity and slimness) Let $\Phi$ be a divisorial monoid on a connected, totally epimorphic category $\mathcal{D}$ let $\mathcal{C} \rightarrow \mathbb{F}_{\Phi}$ be a Frobenioid let $A \in \mathrm{Ob}(\mathcal{C})$ and $A_{\mathcal{D}} \stackrel{\text { def }}{=}$ $\operatorname{Base}(A) \in \operatorname{Ob}(\mathcal{D})$. Suppose further that the category $\mathcal{D}$ is $\operatorname{sim}(c f$. Section 0$)$. Then we have the following.

(i) The composite $\mathcal{C}_{A} \rightarrow \mathcal{D}$ of the natural functor $\mathcal{C}_{A} \rightarrow \mathcal{C}$ with the natural projection functor $\mathcal{C} \rightarrow \mathcal{D}$ is rigid (cf. Section 0 ). In particular, the functor $\mathcal{C} \rightarrow \mathcal{D}$ is rigid.

(ii) The composite $\mathcal{C}_{A} \rightarrow \mathbb{F}_{\Phi}$ of the natural functor $\mathcal{C}_{A} \rightarrow \mathcal{C}$ with the functor $\mathcal{C} \rightarrow \mathbb{F}_{\Phi}$ is rigid. In particular, the functor $\mathcal{C} \rightarrow \mathbb{F}_{\Phi}$ is rigid.

(iii) Suppose, moreover, that every object $A \in \mathrm{Ob}(\mathcal{C})$ satisfies (at least) one of the following two conditions: (a) $\mathcal{O}^{\times}(A)^{\text {imtr-pre }}=\{1\} \quad$ (cf. Proposition 1.9(ii)); (b) $\bigcap_{n \in \mathbb{N}_{\geq 1}}\left\{\mathcal{O}^{\times}(A)\right\}^{n}=\{1\}$, and, moreover, there exists a co-angular pre-step $B \rightarrow A$ (which, by Definition 1.3(iii)(c), induces a bijection $\mathcal{O}^{\times}(B) \stackrel{\sim}{\rightarrow} \mathcal{O}^{\times}(A)$ ) such that $B$ is quasi-Frobenius-trivial and Frobenius-normalized. Then the category $\mathcal{C}$ is slim.

Proof. First, we consider assertion (i). Any automorphism $\alpha$ of the functor $\mathcal{C}_{A} \rightarrow \mathcal{D}$ determines an automorphism of the composite functor $\mathcal{C}_{A}^{\mathrm{pl}-\mathrm{bk}} \rightarrow \mathcal{C}_{A} \rightarrow \mathcal{D}$. On the other hand, this composite functor factors as a composite $\mathcal{C}_{A}^{\text {pl-bk }} \rightarrow \mathcal{D}_{A_{\mathcal{D}}} \rightarrow \mathcal{D}$, where the first functor $\mathcal{C}_{A}^{\mathrm{pl}-\mathrm{bk}} \rightarrow \mathcal{D}_{A_{\mathcal{D}}}$ is (by Definition 1.3(i)(c)) an equivalence of categories. Thus, we conclude that $\alpha$ determines an automorphism of the natural functor $\mathcal{D}_{A_{\mathcal{D}}} \rightarrow \mathcal{D}$, which is necessarily trivial, since $\mathcal{D}$ is slim. Since $A$ is arbitrary, we thus conclude that both $\mathcal{C}_{A} \rightarrow \mathcal{D}$ and $\mathcal{C} \rightarrow \mathcal{D}$ are rigid. This completes the proof of assertion (i).

Next, we consider assertion (ii). Let $\alpha$ be an automorphism of the functor $\mathcal{C}_{A} \rightarrow \mathbb{F}_{\Phi}$. By assertion (i), it follows that the automorphisms of objects of $\mathbb{F}_{\Phi}$ (which, by Proposition 1.5(i), is itself a Frobenioid) induced by $\alpha$ are base-identity automorphisms. Since $\Phi$ is divisorial, hence, in particular, sharp (cf. Definition 1.1(i) and (ii)), it thus follows that all of these automorphisms are trivial, hence that $\alpha$ is trivial. Since $A$ is arbitrary, we thus conclude that both $\mathcal{C}_{A} \rightarrow \mathbb{F}_{\Phi}$ and $\mathcal{C} \rightarrow \mathbb{F}_{\Phi}$ are rigid. This completes the proof of assertion (ii).

Finally, we consider assertion (iii). Let $\alpha$ be an automorphism of the natural functor $\mathcal{C}_{A} \rightarrow \mathcal{C}$. By assertion (i), it follows that the automorphisms of objects of $\mathcal{C}$ induced by $\alpha$ are base-identity automorphisms, i.e., belong to ' $\mathcal{O}^{\times}(-)$'. Moreover, the functoriality of the automorphisms induced by $\alpha$ with respect to isometric pre-steps implies that these automorphisms belong to ' $\mathrm{O}^{\times}(-)^{\text {imtr-pre' }}$. Similarly, the functoriality of the automorphisms induced by $\alpha$ with respect to base-identity endomorphisms implies that, at least in the case of quasi-Frobenius-trivial, Frobenius-normalized objects - hence also (cf. Definition 1.3(iii)(c)) objects as in (b) of the statement of assertion (iii) - these automorphisms belong to $' \bigcap_{n \in \mathbb{N}_{\geq 1}}\left\{\mathcal{O}^{\times}(-)\right\}^{n}$ '. Thus, we conclude that under either of the assumptions (a) or (b) in the statement of assertion (iii), the automorphisms induced by $\alpha$ are trivial. This completes the proof of assertion (iii).

Remark 1.13.1. Note that if the hypothesis of Proposition 1.13(iii) fails to hold, then it is not necessarily the case that $\mathcal{C}$ is slim. Indeed, if $M$ is a perfect pre-divisorial monoid, and $\mathcal{C}$ is a one-object category whose unique object has endomorphism monoid equal to the elementary Frobenioid $\mathbb{F}_{M}$ (so $\mathcal{C}$ equipped with the functor of one-object categories determined by the natural morphism of monoids $\mathbb{F}_{M} \rightarrow \mathbb{F}_{M}$ char is a Frobenioid, by Proposition 1.5(i)), then any collection of elements $\left\{\alpha_{n}\right\}_{n \in \mathbb{N}_{\geq 1}}$ of $M^{ \pm}$such that $\alpha_{n m}=m \cdot \alpha_{n}$ for all $n, m \in \mathbb{N}_{\geq 1}$ 
determines an automorphism of the natural functor $\mathcal{C}_{A} \rightarrow \mathcal{C}$ (which is non-trivial as soon as any of the $\alpha_{n}$ is non-zero) by assigning to an arrow $\phi: B \rightarrow A$ of $\mathcal{C}$ the automorphism $\alpha_{\operatorname{deg}_{\mathrm{Fr}}(\phi)} \in \operatorname{Aut}_{\mathcal{C}}(B)$.

One key result for analyzing the category-theoretic structure of Frobenioids (cf. Section 3) is the following.

PROPOSITION 1.14. (Irreducible morphisms) Let $\Phi$ be a divisorial monoid on a connected, totally epimorphic category $\mathcal{D}$ let $\mathcal{C} \rightarrow \mathbb{F}_{\Phi}$ be a Frobenioid of isotropic type and $\phi \in \operatorname{Arr}(\mathcal{C})$. Suppose further that $\mathcal{D}$ is of FSMFF-type ( $c f$. Section 0 ). Then we have the following.

(i) $\phi$ is irreducible if and only if $\phi$ is one of the following: (a) a prime-Frobenius morphism; (b) a step such that $\operatorname{Div}(\phi)$ is irreducible; (c) a pull-back morphism such that $\operatorname{Base}(\phi)$ is an irreducible morphism of $\mathcal{D}$.

(ii) $\quad \phi$ is a pre-step if and only if it is an FSM-morphism that is mid-adjoint (cf. Section 0) to the irreducible morphisms which are not pre-steps.

(iii) Suppose that $\phi$ is irreducible. Then $\phi$ is a non-pre-step if and only if the following condition holds: there exists an $N \in \mathbb{N}_{\geq 1}$ such that for every equality of composites in $\mathcal{C}$

$$
\alpha_{n} \circ \alpha_{n-1} \circ \cdots \circ \alpha_{2} \circ \alpha_{1}=\psi \circ \phi,
$$

where $\alpha_{1}, \ldots, \alpha_{n}, \psi$ are FSMI-morphisms (cf. Section 0 ), it holds that $n \leq N$.

(iv) Let $\alpha \circ \beta=\beta^{\prime} \circ \alpha^{\prime}$ be an equality of composites of $\mathcal{C}$, where $\operatorname{deg}_{\mathrm{Fr}}(\beta)=\operatorname{deg}_{\mathrm{Fr}}\left(\beta^{\prime}\right)$, and $\alpha, \alpha^{\prime}$ are irreducible. Then $\alpha$ is a prime-Frobenius morphism if and only if $\alpha^{\prime}$ is; moreover, $\operatorname{deg}_{\mathrm{Fr}}(\alpha)=\operatorname{deg}_{\mathrm{Fr}}\left(\alpha^{\prime}\right)$.

(v) Suppose further that $\Phi$ is non-dilating and that $\phi$ is a non-pre-step irreducible endomorphism of a non-group-like object $A \in \mathrm{Ob}(\mathcal{C})$. Then $\phi$ is a Div-identity primeFrobenius endomorphism if and only if the following condition holds: for every step $\alpha: A \rightarrow B$ there exists a non-pre-step irreducible morphism $\psi: B \rightarrow B^{\prime}$ and a step $\beta: B \rightarrow B^{\prime}$ such that $\psi \circ \alpha=\beta \circ \alpha \circ \phi$.

Proof. First, we consider assertion (i). The sufficiency of the condition of assertion (i) follows for morphisms as in (a) (respectively, (b); (c)) from Proposition 1.10(iv) (respectively, the equivalences of categories of Definition 1.3(iii)(d) (cf. also Propositions 1.4(i) and 1.7(v)); Proposition 1.11(vi)). To verify the necessity of the condition of assertion (i), observe that it follows formally from the factorization of Definition 1.3(iv)(a), that $\phi$ is either a morphism of Frobenius type, a step, or a pull-back morphism. Thus, by Propositions 1.7(v), 1.10(iv) and 1.11(vi), the irreducibility of $\phi$ implies immediately that $\phi$ is a morphism as in (a), (b) or $(\mathrm{c})$.

Next, we consider assertion (ii). To verify the sufficiency of the condition of assertion (ii), observe first that by the factorization of Definition 1.3(iv)(a), we may write $\phi=\alpha \circ \beta \circ \gamma$, where $\alpha$ is a pull-back morphism, $\beta$ is a pre-step and $\gamma$ is a morphism of Frobenius type. By assertion (i) (cf. also Proposition 1.10(v)), it follows that $\gamma$ is an isomorphism; thus, we may assume without loss of generality that $\gamma$ is the identity, i.e., $\phi=\alpha \circ \beta$. On the other hand, it follows formally from the fact that $\phi$ is an FSM-morphism that $\alpha$ is fiberwise-surjective (cf. Section 0). Next, I claim that $\alpha$ is a monomorphism. Indeed, write $\phi: A \rightarrow B, \beta: A \rightarrow C, \alpha: C \rightarrow B$ and let $\epsilon_{1}, \epsilon_{2}: D \rightarrow C$ be such that $\alpha \circ \epsilon_{1}=\alpha \circ \epsilon_{2}$. Then by Remark 1.1.1, it follows immediately that $\operatorname{deg}_{\mathrm{Fr}}\left(\epsilon_{1}\right)=\operatorname{deg}_{\mathrm{Fr}}\left(\epsilon_{2}\right)$ and $\operatorname{Div}\left(\epsilon_{1}\right)=\operatorname{Div}\left(\epsilon_{2}\right)$, 
hence, by applying the factorization of Definition 1.3(iv)(a) (and the total epimorphicity of $\mathcal{C}$; cf. also Definition 1.3(ii) and the equivalences of categories of Definition 1.3(iii)(d)), we may assume without loss of generality (from the point of view of showing that $\alpha$ is a monomorphism) that $\epsilon_{1}$ and $\epsilon_{2}$ are pull-back morphisms. Now by 'adding the pullbacks of $\beta_{*}(\operatorname{Div}(\beta))$ via $\epsilon_{1}$ and $\epsilon_{2}$ ' (cf. Proposition 1.11(v); the equivalences of categories of Definition 1.3(iii)(d)), it follows that there exists a pre-step $\zeta: E \rightarrow D$ such that there exist $\gamma_{1}, \gamma_{2} \in \operatorname{Arr}(\mathcal{C})$ satisfying $\epsilon_{1} \circ \zeta=\beta \circ \gamma_{1}$ and $\epsilon_{2} \circ \zeta=\beta \circ \gamma_{2}$. Thus, we have $\phi \circ$ $\gamma_{1}=\alpha \circ \beta \circ \gamma_{1}=\alpha \circ \epsilon_{1} \circ \zeta=\alpha \circ \epsilon_{2} \circ \zeta=\alpha \circ \beta \circ \gamma_{2}=\phi \circ \gamma_{2}$. But since $\phi$ is (an FSMmorphism, hence, in particular) a monomorphism, it follows that $\gamma_{1}=\gamma_{2}$, hence (by the total epimorphicity of $\mathcal{C}$ ) that $\epsilon_{1}=\epsilon_{2}$. This completes the proof of the claim. In particular, we conclude that $\alpha$ is an FSM-morphism.

Thus, it follows (cf. Proposition 1.11(vi)) that Base $(\alpha)$ is an FSM-morphism of $\mathcal{D}$. Since, however, we are operating under the assumption that $\mathcal{D}$ is of FSMFF-type, it follows that if $\alpha$ is not an isomorphism, then $\operatorname{Base}(\alpha)$ admits a subordinate (cf. condition (a) of the definition of a 'category of FSMFF-type' in Section 0) FSMI-morphism, which implies (cf. Proposition 1.11(vi)) that $\alpha$ admits a subordinate FSMI-morphism (which is also a pull-back morphism). Since $\phi$, however, is assumed to be mid-adjoint to the irreducible morphisms which are not pre-steps, we thus obtain a contradiction. Thus, $\alpha$ is an isomorphism, so $\phi$ is a pre-step. This completes the proof of the sufficiency of the condition of assertion (ii). Next, we consider the necessity of the condition of assertion (ii). Thus, suppose that $\phi$ is a pre-step. By Proposition 1.11(vii), $\phi$ is an FSM-morphism; by Proposition 1.7(v), $\phi$ is mid-adjoint to the non-pre-steps. This completes the proof of assertion (ii).

Next, we consider assertion (iii). By assertion (i), it suffices to show that assertion (iii) holds for each of the three types of morphisms '(a), (b), (c)' discussed in assertion (i). If $\phi$ is an irreducible pre-step, then it follows immediately - by taking $\psi$ to be a primeFrobenius morphism of increasingly large Frobenius degree (cf. Proposition 1.10(ii)) - that the condition in the statement of assertion (iii) is false (as desired). On the other hand, if $\phi$ is a non-pre-step, then it is an isometry. Now if the condition in the statement of assertion (iii) is false, then there exist equalities

$$
\alpha_{n} \circ \alpha_{n-1} \circ \cdots \circ \alpha_{2} \circ \alpha_{1}=\psi \circ \phi
$$

where $\alpha_{1}, \ldots, \alpha_{n}, \psi$ are FSMI-morphisms, and $n$ is arbitrarily large. Here, we note that since $\psi \circ \phi$ and $\psi$ are FSM-morphisms, it thus follows formally that $\phi$ is also an FSMmorphism. Next, observe that since $\phi$ is an isometry, it follows from the fact that $\psi$ is irreducible (cf. also assertion (i), Definition 1.1(ii)(b) and Remark 1.1.1) that $\operatorname{Div}(\psi \circ \phi)$ is either zero or irreducible; since, moreover, $\operatorname{deg}_{\mathrm{Fr}}(\psi \circ \phi)$ always divides a product of two prime numbers (cf. assertion (i) and the irreducibility of $\phi$ and $\psi$ ), it thus follows that in any factorization of $\psi \circ \phi$ by FSMI-morphisms, all but three (i.e., corresponding to two possible prime factors of the Frobenius degree, plus one possible irreducible factor of the zero divisor) of the factorizing FSMI-morphisms are pull-back morphisms (cf. assertion (i)). On the other hand, this implies that factorizations of arbitrarily large length determine chains of FSMImorphisms (cf. assertion (i) and Proposition 1.11(vi)) originating from the projection to $\mathcal{D}$ of the domain of $\phi$ which are also of arbitrarily large length, a contradiction (cf. condition (b) of the definition of a 'category of FSMFF-type' in Section 0). This completes the proof of assertion (iii). 
Next, we consider assertion (iv). Since $\operatorname{deg}_{\mathrm{Fr}}(\beta)=\operatorname{deg}_{\mathrm{Fr}}\left(\beta^{\prime}\right)$, it follows from Remark 1.1.1 that $\operatorname{deg}_{\mathrm{Fr}}(\alpha)=\operatorname{deg}_{\mathrm{Fr}}\left(\alpha^{\prime}\right)$, hence (since $\alpha$ and $\alpha^{\prime}$ are irreducible), by assertion (i), that $\alpha$ is a prime-Frobenius morphism if and only if $\alpha^{\prime}$ is. This completes the proof of assertion (iv).

Finally, we consider assertion (v). First, we observe that the necessity of the condition in the statement of assertion (v) (where we take $\psi$ to be a prime-Frobenius morphism such that $\operatorname{deg}_{\mathrm{Fr}}(\phi)=\operatorname{deg}_{\mathrm{Fr}}(\psi)$ ) follows immediately from Proposition 1.10(i) (cf. also Definition 1.3(ii), assertion (i) and the first equivalence of categories of Definition 1.3(iii)(d)). Next, we consider sufficiency. To show that $\phi$ is a prime-Frobenius morphism, it suffices (by assertion (i)) to show that it is not a pull-back morphism. Thus, suppose that $\phi$ is a pull-back morphism. Since $A$ is non-group-like, it follows (cf. Proposition 1.4(iii)) that there exists a step $\alpha: A \rightarrow B$, hence that there exist $\psi$ and $\beta$ as in the statement of assertion (v). By assertions (i) and (iv), $\psi$ is also a pull-back morphism. Write $x \stackrel{\text { def }}{=} \operatorname{Div}(\alpha)$ and $y \stackrel{\text { def }}{=}$ $\alpha^{*}(\operatorname{Div}(\beta))$ (where, for simplicity, we write $\alpha^{*}$ for $\Phi(\operatorname{Base}(\alpha))$ ). Then by Remark 1.1.1, it follows that $\phi^{*}(x+y)=x$, i.e., that $\phi^{*}(x) \leq x$. Since $x \neq 0$ is arbitrary (cf. the first equivalence of categories of Definition 1.3(iii)(d)), it thus follows from our assumption that $\Phi$ is non-dilating that $\phi^{*}$ is the identity morphism. But this implies that $x+y=x$, i.e., (since $\Phi$ is integral - cf. Definition 1.1(i)) that $y=0$, in contradiction to our assumption that $\beta$ is a step (i.e., not just a (necessarily co-angular!) pre-step - cf. Proposition 1.4(i) and (iii)). Thus, we conclude (cf. assertion (iv)) that $\phi$ and $\psi$ are prime-Frobenius morphisms, of the same Frobenius degree. In particular, if we write $x \stackrel{\text { def }}{=} \operatorname{Div}(\alpha)$ and $y \stackrel{\text { def }}{=} \alpha^{*}(\operatorname{Div}(\beta))$, then it follows (cf. Remark 1.1.1) that $\phi^{*}(x+y)=\operatorname{deg}_{\mathrm{Fr}}(\phi) \cdot x$, i.e., that $\phi^{*}(x) \preccurlyeq x$ (cf. Section 0 ), hence (by our assumption that $\Phi$ is non-dilating) that $\phi^{*}$ is the identity morphism. This completes the proof of assertion (v).

\section{Frobenius functors}

In this section, we discuss various functors between Frobenioids that are intended to be reminiscent of the Frobenius morphism in positive characteristic scheme theory.

In the following discussion, we maintain the notation of Section 1. Also, we assume that we have been given a divisorial monoid $\Phi$ on a connected, totally epimorphic category $\mathcal{D}$ and a Frobenioid $\mathcal{C} \rightarrow \mathbb{F}_{\Phi}$.

Proposition 2.1. (The naive Frobenius functor) Let $d \in \mathbb{N}_{\geq 1}$. Then we have the following.

(i) The assignment

$$
A \mapsto A^{\prime}, \quad \phi \mapsto \phi^{\prime},
$$

where $\phi: A \rightarrow B$ is an arbitrary morphism of $\mathcal{C}, \alpha: A \rightarrow A^{\prime}, \beta: B \rightarrow B^{\prime}$ are morphisms of Frobenius type of Frobenius degree $d$ and $\phi^{\prime}$ is the unique morphism such that $\phi^{\prime} \circ \alpha=\beta \circ \phi$ (cf. Proposition 1.10(i)), and determines a functor

$$
\Psi: \mathcal{C} \rightarrow \mathcal{C}
$$

(well defined up to isomorphism of functors) which we shall refer to as the naive Frobenius functor (of degree $d$ ) on $\mathcal{C}$. Finally, the composite of the naive Frobenius functor of degree $d_{1} \in \mathbb{N}_{\geq 1}$ on $\mathcal{C}$ with the naive Frobenius functor of degree $d_{2} \in \mathbb{N}_{\geq 1}$ on $\mathcal{C}$ is isomorphic to the naive Frobenius functor of degree $d_{1} \cdot d_{2}$ on $\mathcal{C}$. 
(ii) The functor $\Psi$ of (i) is '1-compatible', relative to $\mathcal{C} \rightarrow \mathbb{F}_{\Phi}$, with the functor $\mathbb{F}_{\Phi} \rightarrow$ $\mathbb{F}_{\Phi}-$ which we shall refer to as the Frobenius functor on $\mathbb{F}_{\Phi}$ - determined (cf. Definition 1.1(iii)) by the endomorphism of the functor $\Phi$ given by multiplication by d. Moreover, if, in the notation of (i), $A=A^{\prime}, A$ is Frobenius-normalized and the morphism $\alpha: A \rightarrow A^{\prime}$ is taken to be a base-identity endomorphism, then the morphism of monoids $\mathcal{O}^{\triangleright}(A) \rightarrow \mathcal{O}^{\triangleright}\left(A^{\prime}\right)$ induced by $\Psi$ is given by raising to the $d$ th power.

(iii) $\mathcal{C}$ is of perfect type if and only if $\Psi$ is an equivalence of categories.

Proof. Assertions (i) and (ii) follow immediately from Definition 1.3(ii) and Proposition 1.10(i) (cf. also Proposition 1.7(i)). Finally, we consider assertion (iii). The sufficiency of the condition of assertion (iii) follows immediately from the definition of 'perfect' (cf. Definition 1.2(iv) and Remark 1.1.1). To verify necessity, suppose that $\mathcal{C}$ is of perfect type. Then the essential surjectivity of $\Psi$ follows immediately from the definition of 'perfect' (cf. Definition 1.2(iv)). To verify that $\Psi$ is fully faithful, we reason as follows: in light of the 1-compatibility of $\Psi$ with the Frobenius functor on $\mathbb{F}_{\Phi}$ (cf. assertion (ii)), the total epimorphicity of $\mathcal{C}$, and the factorization of Definition 1.3(iv)(a), it follows immediately that one may reduce to the case of linear morphisms by applying the existence and (essential) uniqueness of morphisms of Frobenius type of a given Frobenius degree (cf. Definition 1.3(ii)). Moreover, by applying the equivalence of categories (involving pullbacks) of Definition 1.3(i)(c) (cf. also the isomorphism of functors appearing in the definition of a 'pull-back morphism' in Definition 1.2(ii)), one may reduce further to the case of presteps. But the case of pre-steps follows immediately from the definition of 'perfect' (cf. Definition 1.2(iv)). This completes the proof of assertion (iii).

Remark 2.1.1. If $\mathcal{C}$ is of perfect type, then for any $d=a / b \in \mathbb{Q}_{>0}$, where $a, b \in \mathbb{N}_{\geq 1}$, composing the naive Frobenius functor of degree $a$ with some quasi-inverse functor to the naive Frobenius functor of degree $b$ yields a 'naive Frobenius functor of degree d', which, by Proposition 2.1(i), is independent of the choice of $a$ and $b$.

PROPOSITION 2.2. (The functor $\mathcal{O}^{\triangleright}(-)$ ) Write $\mathcal{D}^{*}$ for the category whose objects are the objects of $\mathcal{C}^{\text {istr }}$ and whose morphisms are given as

$$
\operatorname{Hom}_{\mathcal{D}^{*}}(A, B) \stackrel{\text { def }}{=} \operatorname{Hom}_{\mathcal{D}}\left(A_{\mathcal{D}}, B_{\mathcal{D}}\right)
$$

(where $A, B \in \mathrm{Ob}\left(\mathcal{C}^{\text {istr }}\right), A_{\mathcal{D}} \stackrel{\text { def }}{=} \mathrm{Base}(A)$ and $\left.B_{\mathcal{D}} \stackrel{\text { def }}{=} \mathrm{Base}(B)\right)$. Thus, the natural projection functor $\mathcal{C} \rightarrow \mathcal{D}$ determines natural functors $\mathcal{C}^{\text {istr }} \rightarrow \mathcal{D}^{*} \rightarrow \mathcal{D}$. Moreover we have the following.

(i) The functor $\mathcal{D}^{*} \rightarrow \mathcal{D}$ is an equivalence of categories.

(ii) There is a unique contravariant functor

$$
\begin{gathered}
\mathcal{D}^{*} \rightarrow \text { Mon } \\
\mathrm{Ob}\left(\mathcal{C}^{\text {istr }}\right)=\mathrm{Ob}\left(\mathcal{D}^{*}\right) \ni A \mapsto \mathcal{O}^{\triangleright}(A) \in \mathrm{Ob}(\mathfrak{M o n})
\end{gathered}
$$

such that for $\phi: A \rightarrow B$ in $\operatorname{Arr}\left(\mathcal{C}^{\text {istr }}\right)$, with image $\phi_{\mathcal{D}^{*}}$ in $\mathcal{D}^{*}$, the following properties are satisfied: (a) if $\phi$ is a (necessarily co-angular - cf. Proposition 1.4(i)) linear morphism, then $\mathcal{O}^{\triangleright}\left(\phi_{\mathcal{D}^{*}}\right): \mathcal{O}^{\triangleright}(B) \rightarrow \mathcal{O}^{\triangleright}(A)$ is the inclusion of Proposition 1.11(iv); (b) if $\phi$ is a (necessarily co-angular) pre-step, then $\mathcal{O}^{\triangleright}\left(\phi_{\mathcal{D}^{*}}\right): \mathcal{O}^{\triangleright}(B) \rightarrow \mathcal{O}^{\triangleright}(A)$ is 
the bijection of Definition 1.3(iii)(c). By abuse of notation, we shall also denote by ' $\mathcal{O}^{\triangleright}(-)$ ' the restriction of this functor on $\mathcal{D}^{*}$ to $\left(\mathcal{C}^{\text {istr }}\right)^{\text {lin }}$. Finally, by applying the equivalence of categories of (i), we obtain a contravariant functor $\mathcal{D} \rightarrow \mathfrak{M o n}$, which, by abuse of notation, we shall also denote by ' $\mathrm{O}^{\triangleright}(-)$ ', and which is well defined up to isomorphism.

(iii) The assignment $\mathrm{Ob}\left(\mathcal{C}^{\text {istr }}\right) \ni A \mapsto \mathcal{O}^{\times}(A)\left(\subseteq \mathcal{O}^{\triangleright}(A)\right)$ determines a subfunctor of the functor of (ii) which is equal to the subfunctor $A \mapsto \mathcal{O}^{\triangleright}(A)^{ \pm}$(cf. the notation of Section 0). Moreover, the operation 'Div(-)' determines a functorial homomorphism

$$
\mathcal{O}^{\triangleright}(A) \rightarrow \Phi(A)
$$

that induces an inclusion $\mathcal{O}^{\triangleright}(A)^{\text {char }}=\mathcal{O}^{\triangleright}(A) / \mathcal{O}^{\times}(A) \hookrightarrow \Phi(A)$ (cf. the notation of Section 0$)$.

(iv) If $\phi: A \rightarrow A^{\text {istr }}$ is an isotropic hull in $\mathcal{C}$, then $\phi$ determines a natural inclusion of monoids $\mathcal{O}^{\triangleright}(A) \hookrightarrow \mathcal{O}^{\triangleright}\left(A^{\text {istr }}\right)$.

Proof. As for assertion (i), essential surjectivity follows immediately from Definition 1.3(i)(a) (i.e., applied to the Frobenioid $\mathcal{C}^{\text {istr }}$ - cf. Proposition 1.9(v)), while fully faithfulness follows formally from the definition of the category $\mathcal{D}^{*}$.

Next, we consider assertion (ii). Let $A, B \in \mathrm{Ob}\left(\mathcal{C}^{\text {istr }}\right), A_{\mathcal{D}} \stackrel{\text { def }}{=} \operatorname{Base}(A)$ and $B_{\mathcal{D}} \stackrel{\text { def }}{=}$ $\operatorname{Base}(B)$. Now observe that any morphism $A_{\mathcal{D}} \rightarrow B_{\mathcal{D}}$ in $\mathcal{D}$ factors as the composite of an isomorphism $A_{\mathcal{D}} \stackrel{\sim}{\rightarrow} C_{\mathcal{D}}$, where $C \in \mathrm{Ob}\left(\mathcal{C}^{\text {istr }}\right), C_{\mathcal{D}} \stackrel{\text { def }}{=} \operatorname{Base}(C)$, with a morphism $C_{\mathcal{D}} \rightarrow B_{\mathcal{D}}$ which is the projection to $\mathcal{D}$ of a pull-back morphism $C \rightarrow B$ of $\mathcal{C}^{\text {istr }}$ (cf. Definition 1.3(i)(c)); moreover, this pull-back morphism is uniquely determined, as an object of $\mathcal{C}_{B}^{\text {istr }}$, up to isomorphism (cf. the isomorphism of functors appearing in the definition of a 'pull-back morphism' in Definition 1.2(ii)). Thus, it follows that to construct the desired functor ' $\mathcal{O}^{\triangleright}(-)$ ' on $\mathcal{D}^{*}$, it suffices to construct, for each isomorphism $\phi_{\mathcal{D}}: A_{\mathcal{D}} \rightarrow$ $B_{\mathcal{D}}$, a bijection $\mathcal{O}^{\triangleright}\left(\phi_{\mathcal{D}}\right): \mathcal{O}^{\triangleright}(A) \stackrel{\sim}{\rightarrow} \mathcal{O}^{\triangleright}(B)$ which is compatible with composition of isomorphisms. (Indeed, once one constructs ' $\mathcal{O}^{\triangleright}(-)$ ' in this fashion, the fact that this ' $\mathcal{O}{ }_{(-)}$' is compatible with composites of morphisms of $\mathcal{D}^{*}$ follows immediately from the manifest functoriality of the inclusion of Proposition 1.11(iv).) This may be done by using co-angular pre-steps $\gamma_{A}: C \rightarrow A$ and $\gamma_{B}: C \rightarrow B$ such that $\phi_{\mathcal{D}}=\operatorname{Base}\left(\gamma_{B}\right) \circ \operatorname{Base}\left(\gamma_{A}\right)^{-1}$ (cf. Definition 1.3(i)(b)) and the bijections $\mathcal{O}^{\triangleright}\left(\gamma_{A}\right): \mathcal{O}^{\triangleright}(A) \stackrel{\sim}{\rightarrow} \mathcal{O}^{\triangleright}(C)$ and $\mathcal{O}^{\triangleright}\left(\gamma_{B}\right)$ : $\mathcal{O}^{\triangleright}(B) \stackrel{\sim}{\rightarrow} \mathcal{O}^{\triangleright}(C)$ determined by $\gamma_{A}$ and $\gamma_{B}$ (cf. Definition 1.3(iii)(c)). Note, moreover, that the resulting bijection $\mathcal{O}^{\triangleright}\left(\gamma_{A}\right)^{-1} \circ \mathcal{O}^{\triangleright}\left(\gamma_{B}\right)$ is independent of the choice of $\gamma_{A}$ and $\gamma_{B}$. (Indeed, if $\delta_{A}: D \rightarrow A$ and $\delta_{B}: D \rightarrow B$ satisfy $\phi_{\mathcal{D}}=\operatorname{Base}\left(\delta_{B}\right) \circ \operatorname{Base}\left(\delta_{A}\right)^{-1}$, then there exist (cf. Definition 1.3(i)(b)) co-angular pre-steps $\epsilon_{C}: E \rightarrow C$ and $\epsilon_{D}: E \rightarrow D$ such that

$$
\operatorname{Base}\left(\gamma_{A}\right) \circ \operatorname{Base}\left(\epsilon_{C}\right)=\operatorname{Base}\left(\delta_{A}\right) \circ \operatorname{Base}\left(\epsilon_{D}\right),
$$

which (since $\left.\operatorname{Base}\left(\delta_{B}\right) \circ \operatorname{Base}\left(\delta_{A}\right)^{-1}=\operatorname{Base}\left(\gamma_{B}\right) \circ \operatorname{Base}\left(\gamma_{A}\right)^{-1}\right)$ implies that

$$
\operatorname{Base}\left(\gamma_{B}\right) \circ \operatorname{Base}\left(\epsilon_{C}\right)=\operatorname{Base}\left(\delta_{B}\right) \circ \operatorname{Base}\left(\epsilon_{D}\right)
$$

hence that

$$
\mathcal{O}^{\triangleright}\left(\gamma_{A} \circ \epsilon_{C}\right)=\mathcal{O}^{\triangleright}\left(\delta_{A} \circ \epsilon_{D}\right), \quad \mathcal{O}^{\triangleright}\left(\gamma_{B} \circ \epsilon_{C}\right)=\mathcal{O}^{\triangleright}\left(\delta_{B} \circ \epsilon_{D}\right)
$$


(cf. Definition 1.3(iii)(c)), i.e., that

$$
\mathcal{O}^{\triangleright}\left(\epsilon_{C}\right) \circ \mathcal{O}^{\triangleright}\left(\gamma_{A}\right)=\mathcal{O}^{\triangleright}\left(\epsilon_{D}\right) \circ \mathcal{O}^{\triangleright}\left(\delta_{A}\right), \quad \mathcal{O}^{\triangleright}\left(\epsilon_{C}\right) \circ \mathcal{O}^{\triangleright}\left(\gamma_{B}\right)=\mathcal{O}^{\triangleright}\left(\epsilon_{D}\right) \circ \mathcal{O}^{\triangleright}\left(\delta_{B}\right),
$$

that is to say

$$
\mathcal{O}^{\triangleright}\left(\gamma_{A}\right)^{-1} \circ \mathcal{O}^{\triangleright}\left(\gamma_{B}\right)=\mathcal{O}^{\triangleright}\left(\delta_{A}\right)^{-1} \circ \mathcal{O}^{\triangleright}\left(\delta_{B}\right)
$$

as desired.) This completes the proof of assertion (ii).

Assertion (iii) is immediate from the definitions (cf. also Definition 1.3(iii)(b) and Definition 1.3(vi)).

Assertion (iv) follows immediately from the 'universal property of an isotropic hull' (cf. Definition 1.2(iv)) and the fact that an isotropic hull is always a monomorphism (cf. Definition 1.3(v)(a)).

Definition 2.3. We shall refer to as a characteristic splitting on $\mathcal{C}$ a subfunctor in monoids

$$
\tau:\left(\mathcal{C}^{\text {istr }}\right)^{\text {lin }} \rightarrow \mathfrak{M o n}
$$

of the functor $\mathcal{O}^{\triangleright}(-):\left(\mathcal{C}^{\text {istr }}\right)^{\text {lin }} \rightarrow \mathfrak{M o n}$ of Proposition 2.2(ii), such that the following properties hold: (a) for every $A \in \mathrm{Ob}\left(\mathcal{C}^{\text {istr }}\right), \tau(A)$ maps bijectively onto $\mathcal{O}^{\triangleright}(A)^{\text {char }}$, hence determines a splitting of monoids

$$
\mathcal{O}^{\times}(A) \times \tau(A) \stackrel{\sim}{\rightarrow} \mathcal{O}^{\triangleright}(A)
$$

which is functorial in $A$; (b) for every isotropic hull $A \rightarrow A^{\text {istr }}$ of $\mathcal{C}, \tau\left(A^{\text {istr }}\right) \subseteq \mathcal{O}^{\triangleright}\left(A^{\text {istr }}\right)$ lies in the image of $\mathcal{O}^{\triangleright}(A)$ via the natural injection of Proposition 2.2(iv).

\section{Definition 2.4.}

(i) We shall say that $M \in \mathrm{Ob}(\mathfrak{M o n})$ is perf-factorial if it satisfies the following conditions.

(a) $M$ is divisorial.

(b) For every $\mathfrak{p} \in \operatorname{Prime}(M)$ (cf. Section 0), the monoid $M_{\mathfrak{p}}$ is monoprime (cf. Section 0).

(c) The map

$$
\begin{gathered}
M^{\mathrm{pf}} \rightarrow M_{\text {factor }}^{\mathrm{rlf}} \stackrel{\text { def }}{=} \prod_{\mathfrak{p} \in \operatorname{Prime}(M)} M_{\mathfrak{p}}^{\mathrm{rlf}} \\
a \mapsto\left(\ldots, \sup \left(\operatorname{Bound}_{\mathfrak{p} \cup\{0\}}(a)\right), \ldots\right)
\end{gathered}
$$

(where we write $M_{\mathfrak{p}}^{\text {rlf }} \stackrel{\text { def }}{=} M_{\mathfrak{p}}^{\text {pf }} \otimes \mathbb{R}_{\geq 0}$, we refer to Section 0 for more on the notation ' $M^{\mathrm{pf}},{ }^{\mathrm{p}}, M_{\mathfrak{p}}^{\mathrm{pf}}$ ' and ' $\otimes \mathbb{R}_{\geq 0}$ ' and the 'sup' at the index $\mathfrak{p}$ is taken in $\left.M_{\mathfrak{p}}^{\mathrm{rlf}}\right)$ is a well-defined (i.e., the various Bound $\bar{p}_{\mathfrak{p}} \cup\{0\}(a) \subseteq M_{\mathfrak{p}}^{\text {rlf }}$ are bounded subsets) injective homomorphism of monoids whose image lies in $\prod_{\mathfrak{p} \in \operatorname{Prime}(M)} M_{\mathfrak{p}}^{\mathrm{pf}}$, hence determines an injective homomorphism

$$
M^{\mathrm{pf}} \hookrightarrow M_{\text {factor }}^{\mathrm{pf}} \stackrel{\text { def }}{=} \prod_{\mathfrak{p} \in \operatorname{Prime}(M)} M_{\mathfrak{p}}^{\mathrm{pf}}
$$

which we shall refer to as the factorization homomorphism of $M^{\mathrm{pf}}$. We shall often use the factorization homomorphism to regard $M^{\mathrm{pf}}$ as a submonoid of $M_{\text {factor }}^{\mathrm{pf}} \subseteq M_{\text {factor }}^{\mathrm{rlf}}$. 
(d) If $a \in M_{\text {factor }}^{\mathrm{rlf}}$, then we shall write $\operatorname{Supp}(a) \subseteq \operatorname{Prime}(M)$ for the subset of $\mathfrak{p}$ for which the component of $a$ at $\mathfrak{p}$ is non-zero and refer to $\operatorname{Supp}(a)$ as the support of $a$. Then the submonoid $M^{\mathrm{pf}} \subseteq M_{\text {factor }}^{\mathrm{pf}}$ satisfies the following property: if $a \in M_{\text {factor }}^{\mathrm{pf}}$ and $b \in M^{\mathrm{pf}}$ satisfy $\operatorname{Supp}(a) \subseteq \operatorname{Supp}(b)$, then $a \in M^{\mathrm{pf}}$. (Thus, in particular, if $a, b \in M^{\mathrm{pf}}$, then an inequality ' $a \leq b$ ' holds in $M^{\text {pf }}$ if and only if it holds in $M_{\text {factor }}^{\mathrm{pf}}$.)

Now suppose that $M$ is perf-factorial. Then we shall refer to the (subset which is easily verified to be a) submonoid

$$
M^{\text {rlf }} \subseteq M_{\text {factor }}^{\text {rlf }}
$$

of elements $a \in M_{\text {factor }}^{\text {rlf }}$ such that there exists a, $b \in M^{\text {pf }}$ satisfying $\operatorname{Supp}(a) \subseteq \operatorname{Supp}(b)$ as the realification of $M$. Thus, both the submonoid $M^{\mathrm{pf}} \subseteq M_{\text {factor }}^{\mathrm{pf}}$ and the submonoid $M^{\mathrm{rlf}} \subseteq$ $M_{\text {factor }}^{\text {rlf }}$ are completely determined by the collection of subsets $\operatorname{Supp}(a) \subseteq \operatorname{Prime}(M)$, as $a$ ranges over the elements of $M^{\mathrm{pf}}$; if $a, b \in M^{\mathrm{rlf}}$, then an inequality ' $a \leq b$ ' holds in $M^{\mathrm{rlf}}$ if and only if it holds in $M_{\text {factor }}^{\text {rlf }}$;

$$
\left(M^{\mathrm{rlf}}\right)^{\mathrm{gp}} \subseteq\left(M_{\mathrm{factor}}^{\mathrm{rlf}}\right)^{\mathrm{gp}}=\prod_{\mathfrak{p} \in \operatorname{Prime}(M)}\left(M_{\mathfrak{p}}^{\mathrm{rlf}}\right)^{\mathrm{gp}}
$$

is an $\mathbb{R}$-vector space. Finally, one verifies immediately that $M^{\mathrm{pf}}$ and $M^{\mathrm{rlf}}$ are also perffactorial.

(ii) Let $\Lambda$ be a monoid type. Then we shall say that $\Lambda$ supports $M \in \mathrm{Ob}(\mathfrak{M}$ n) if any of the following conditions hold: (a) $\Lambda=\mathbb{Z}$; (b) $\Lambda=\mathbb{Q}$ and $M$ is perfect; (c) $\Lambda=\mathbb{R}, M$ is perfect and perf-factorial and for every $\mathfrak{p} \in \operatorname{Prime}(M)$, the monoid $M_{\mathfrak{p}}$ is $\mathbb{R}$-monoprime. Note that if $\Lambda$ supports $M$, then $\Lambda_{>0}$ acts naturally on $M$.

(iii) Let $\Lambda$ be a monoid type that supports $\Phi$ (cf. Definition 1.1(ii)) and let $d \in \Lambda_{>0}$. Then we shall write

$$
d \cdot \Phi(-) \subseteq \Phi(-)
$$

for the subfunctor of $\Phi$ determined by the assignment $\mathrm{Ob}\left(\left(\mathcal{C}^{\text {istr }}\right)^{\operatorname{lin}}\right) \ni A \mapsto d \cdot(\Phi(A))(\subseteq$ $\Phi(A))$ and

$$
\mathcal{C}^{(d)} \subseteq \mathcal{C}
$$

for the subcategory determined by the arrows whose zero divisor lies in $d \cdot \Phi(-) \subseteq \Phi(-)$. Finally, multiplication by $d$ on $\Phi(-)$ determines a 'Frobenius functor' (associated to $d-\mathrm{cf}$. Proposition 2.1(ii))

$$
\mathbb{F}_{\Phi} \rightarrow \mathbb{F}_{\Phi}
$$

which is compatible with Frobenius degrees and the natural projection functor $\mathbb{F}_{\Phi} \rightarrow \mathcal{D}$.

Proposition 2.5. (The unit-linear Frobenius functor) Let $\tau$ be a characteristic splitting on $\mathcal{C}$, let $\Lambda$ be a monoid type that supports $\Phi$ and let $d \in \Lambda_{>0}$. Suppose that the Frobenioid $\mathcal{C}$ is of Frobenius-normalized, metrically trivial and Aut-ample type. Then we have the following.

(i) The natural inclusion $\mathcal{O}^{\triangleright}(A)^{\text {char }} \hookrightarrow \Phi(A)$, where $A \in \mathrm{Ob}\left(\mathcal{C}^{\text {istr }}\right)$, of Proposition 2.2(iii), is, in fact, a bijection.

(ii) $\mathcal{C}^{\text {istr }}$ is of base-trivial type. Moreover, every object of $\mathcal{C}^{\text {istr }}$ is Frobenius-trivial. 
(iii) There exists an equivalence of categories

$$
\Psi: \mathcal{C} \stackrel{\sim}{\rightarrow} \mathcal{C}^{(d)},
$$

which we shall refer to as the unit-linear Frobenius functor (associated to $\tau$ and d), that satisfies the following properties: (a) $\Psi$ acts as the identity on objects and isometries of $\mathcal{C}$; (b) $\Psi$ is 1-compatible, relative to the functors

$$
\mathcal{C} \rightarrow \mathbb{F}_{\Phi}, \quad \mathcal{C}^{(d)} \rightarrow \mathbb{F}_{d \cdot \Phi}=\left(\mathbb{F}_{\Phi}\right)^{(d)} \subseteq \mathbb{F}_{\Phi}
$$

with the Frobenius functor associated to $d$ on $\mathbb{F}_{\Phi}$ (which implies, in particular, that $\mathcal{C}^{(d)}$, equipped with the natural functor $\mathcal{C}^{(d)} \rightarrow \mathbb{F}_{d \cdot \Phi}$, is a Frobenioid).

Proof. First, we observe that, by applying either of the equivalences of categories of Definition 1.3(iii)(d), assertion (i) follows formally from the fact that $\mathcal{C}$ is of metrically trivial and Aut-ample type.

Next, we consider assertion (ii). Since $\mathcal{C}$ is of metrically trivial type, it follows from the existence of (necessarily co-angular - cf. Proposition 1.4(i)) pre-steps relating baseisomorphic objects of $\mathcal{C}^{\text {istr }}$ (cf. Definition 1.3(i)(b)), that the isomorphism class of an object of $\mathcal{C}^{\text {istr }}$ is completely determined by the isomorphism class of $\mathcal{D}$ to which it projects. In particular, it follows from the existence of Frobenius-trivial objects (cf. Definition 1.3(i)(a)) that every object of $\mathcal{C}^{\text {istr }}$ is Frobenius-trivial. This completes the proof of assertion (ii).

Finally, we consider assertion (iii). By applying the factorizations of Definition 1.3(iv)(a) and (v)(c), together with the bijection of assertion (i) (cf. also the equivalences of categories of Definition 1.3(iii)(d)), we conclude that every morphism $\phi$ of $\mathcal{C}$ admits a factorization

$$
\phi=\alpha \circ \beta \circ \gamma \circ \delta
$$

in $\mathcal{C}$, where $\alpha$ is a pull-back morphism, $\beta$ is a base-identity pre-step endomorphism (hence is co-angular, by Definition 1.3(iii)(b)), $\gamma$ is an isometric pre-step and $\delta$ is a morphism of Frobenius type. Moreover, this factorization is unique (cf. Definition 1.3(iv)(a) and (v)(c)), up to replacing $(\alpha, \beta, \gamma, \delta)$ by $\left(\alpha \circ \epsilon, \epsilon^{-1} \circ \beta \circ \zeta, \zeta^{-1} \circ \gamma \circ \theta, \theta^{-1} \circ \delta\right)$, where $\epsilon, \theta$ are isomorphisms of $\mathcal{C}$ and $\zeta=\beta^{\prime} \circ \epsilon$, for some base-identity automorphism $\beta^{\prime}$. Suppose that $\beta \in \mathcal{O}^{\triangleright}(A)$, for $A \in \operatorname{Ob}(\mathcal{C})$. Thus, by applying the characteristic splitting

$$
\mathcal{O}^{\times}(A) \times \tau(A) \stackrel{\sim}{\rightarrow} \mathcal{O}^{\triangleright}(A)
$$

(which applies even if $A$ is not isotropic - cf. Definition 2.3(a) and (b)) to $\beta \in \mathcal{O}^{\triangleright}(A)$, we obtain a factorization

$$
\beta=\beta_{0} \cdot \beta_{1}
$$

(where $\beta_{0} \in \mathcal{O}^{\times}(A)$ and $\beta_{1} \in \tau(A)$ ). Now we set

$$
\Psi(\beta) \stackrel{\text { def }}{=} \beta_{0} \cdot \beta_{1}^{d}, \quad \Psi(\phi) \stackrel{\text { def }}{=} \alpha \circ \Psi(\beta) \circ \gamma \circ \delta
$$

(where we note that the expression ' $\beta_{1}^{d}$ ' makes sense for $d \in \Lambda_{>0}$, by assertion (i) and Definition 2.4(ii)). Then it follows immediately from the functoriality of the characteristic splitting $\tau(-)$ that for any isomorphism $\epsilon: A^{\prime} \stackrel{\sim}{\rightarrow} A$ in $\mathcal{C}, \beta^{\prime} \in \mathcal{O}^{\times}(A)$, we have $\Psi\left(\epsilon^{-1} \circ\right.$ $\left.\beta \circ \beta^{\prime} \circ \epsilon\right)=\epsilon^{-1} \circ \Psi(\beta) \circ \beta^{\prime} \circ \epsilon$. This implies immediately that $\Psi(\phi)$ is independent of the choice of factorization $\phi=\alpha \circ \beta \circ \gamma \circ \delta$. 
Next, observe that by assertion (ii), it follows that if $\phi \in \operatorname{Arr}\left(\mathcal{C}^{\text {istr }}\right)$, then the morphism of Frobenius type $\delta$ may be taken to be a base-identity endomorphism. Thus, by the functoriality of $\tau$ with respect to morphisms of $\left(\mathcal{C}^{\text {istr }}\right)^{\text {lin }}$, and our assumption that $\mathcal{C}$ is of Frobeniusnormalized type - together with the elementary computation

$$
\Psi\left(\beta^{d^{\prime}}\right)=\Psi\left(\beta_{0}^{d^{\prime}} \cdot \beta_{1}^{d^{\prime}}\right)=\beta_{0}^{d^{\prime}} \cdot\left(\beta_{1}^{d^{\prime}}\right)^{d}=\left(\beta_{0} \cdot \beta_{1}^{d}\right)^{d^{\prime}}=\Psi(\beta)^{d^{\prime}}
$$

for $d^{\prime} \in \mathbb{N}_{\geq 1}$ - it follows that the assignment $\phi \mapsto \Psi(\phi)$ is compatible with composites, at least when $\phi \in \operatorname{Arr}\left(\mathcal{C}^{\text {istr }}\right)$. On the other hand, since isotropic hulls are monomorphisms (cf. Definition 1.3(v)(a)), this implies (by relating an arbitrary $\phi \in \operatorname{Arr}(\mathcal{C})$ to the result of applying the isotropification functor of Proposition 1.9(v) to $\phi$ ) that the assignment $\phi \mapsto \Psi(\phi)$ is compatible with composites, for arbitrary $\phi \in \operatorname{Arr}(\mathcal{C})$. This completes the definition of a functor $\Psi: \mathcal{C} \rightarrow \mathcal{C}^{(d)}$ which satisfies properties (a) and (b) in the statement of Proposition 2.5(iii). On the other hand, it is clear from the definition of $\Psi$ and $\mathcal{C}^{(d)}$ that $\Psi$ is essentially surjective, faithful and full (cf. assertion (i)). This completes the proof of Proposition 2.5.

Remark 2.5.1. If $\mathcal{C}$ is of isotropic and unit-trivial type, then the 'unit-linear Frobenius functor' of Proposition 2.5(iii) may be regarded as a sort of generalization of the 'naive Frobenius functor' of Proposition 2.1(i) to the case of $d \notin \mathbb{N}_{\geq 1}$.

COROLlaRY 2.6. (Unit-wise Frobenius functors I) Let $\tau$ be $a$ characteristic splitting on $\mathcal{C}$ and let $d \in \mathbb{N}_{\geq 1}$. Suppose that the Frobenioid $\mathcal{C}$ is of Frobenius-normalized, metrically trivial and Aut-ample type. Then there exists a functor

$$
\Psi: \mathcal{C} \rightarrow \mathcal{C}
$$

which we shall refer to as the unit-wise Frobenius functor (associated to $\tau$ and $d$ ), which satisfies the following properties.

(a) $\Psi$ is 1-compatible, relative to the functor $\mathcal{C} \rightarrow \mathbb{F}_{\Phi}$, with the identity functor on $\mathbb{F}_{\Phi}$.

(b) $\Psi$ maps an object (respectively, morphism of Frobenius type; pre-step; pull-back morphism) of $\mathcal{C}^{\text {istr }}$ to an isomorphic object (respectively, abstractly equivalent morphism; abstractly equivalent morphism; abstractly equivalent morphism) of $\mathcal{C}$.

(c) If $A \in \mathrm{Ob}\left(\mathcal{C}^{\mathrm{istr}}\right)$, then there exists an isomorphism $\Psi(A) \cong A$ such that the endomorphism of $\mathcal{O}^{\times}(A)$ induced by $\Psi$ followed by conjugation by this isomorphism is given by raising to the $d$ th power.

(d) If $\mathcal{C}$ is of perfect type, then $\Psi$ is an equivalence of categories. If $d=1$ or $\mathcal{C}$ is of isotropic and unit-trivial type, then $\Psi$ is isomorphic to the identity functor.

Proof. First, let us observe that the naive Frobenius functor $\mathcal{C} \rightarrow \mathcal{C}$ associated to $d$ (cf. Proposition 2.1(i)) factors naturally through the subcategory $\mathcal{C}^{(d)} \subseteq \mathcal{C}$ (cf. Proposition 2.1(ii) and Definition 2.4(iii)) and write $\Psi_{1}: \mathcal{C} \rightarrow \mathcal{C}^{(d)}$ for the resulting functor. Next, let us write $\Psi_{2}: \mathcal{C}^{(d)} \rightarrow \mathcal{C}$ for some quasi-inverse functor to the unit-linear Frobenius functor (which is an equivalence of categories) associated to $d$ (cf. Proposition 2.5(iii)). Set $\Psi \stackrel{\text { def }}{=} \Psi_{2} \circ \Psi_{1}$ : $\mathcal{C} \rightarrow \mathcal{C}$. Then it follows immediately from Propositions 2.1(ii) and 2.5(iii)(b) that $\Psi$ satisfies property (a). Since (cf. Proposition 2.5(ii)) the isomorphism class of an object of $\mathcal{C}^{\text {istr }}$ is completely determined by the isomorphism class of $\mathcal{D}$ to which it projects, it thus follows that $\Psi$ preserves isomorphism classes of objects of $\mathcal{C}^{\text {istr }}$. Now the remainder of properties (b), 
(c) and (d) follow immediately from the construction of $\Psi_{1}$ and $\Psi_{2}$ in the proofs of Propositions 2.1(i) and 2.5(iii) (cf. also Remark 2.5.1, Proposition 1.10(i), Definition 1.3(ii), equivalences of categories of Definition 1.3(iii)(d), the definition of a 'pull-back morphism' in Definition 1.2(ii) and Proposition 2.1(iii)).

Definition 2.7. Suppose that the Frobenioid $\mathcal{C}$ is of isotropic type.

(i) We shall refer to as a base-section of the Frobenioid $\mathcal{C}$ any subcategory $\mathcal{P} \subseteq \mathcal{C}^{\text {pl-bk }} \subseteq \mathcal{C}$ (where $\mathcal{C}^{\text {pl-bk }} \subseteq \mathcal{C}$ is as in Definition 1.3(i)(c)) satisfying the following conditions: (a) $\mathcal{P}$ is a skeleton (cf. Section 0); (b) every object of $\mathcal{P}$ is Frobenius-trivial; (c) the composite $\mathcal{P} \rightarrow \mathcal{D}$ of the inclusion functor $\mathcal{P} \hookrightarrow \mathcal{C}$ with the natural projection functor $\mathcal{C} \rightarrow \mathcal{D}$ is an equivalence of categories. In this situation, we shall refer to the morphisms of $\mathcal{C}$ that lie in $\mathcal{P}$ as $\mathcal{P}$ distinguished.

(ii) Let $\mathcal{P} \subseteq \mathcal{C}$ be a base-section. Observe that since $\mathcal{D}$, hence also $\mathcal{P}$, is a connected category, it follows immediately that for any $\epsilon \in \operatorname{End}(\mathcal{P} \hookrightarrow \mathcal{C})$, it makes sense to speak of the Frobenius degree $\operatorname{deg}_{\mathrm{Fr}}(\epsilon) \in \mathbb{N}_{\geq 1}$ of $\epsilon$-i.e., the Frobenius degree of the endomorphisms in $\mathcal{C}$ (of objects of $\mathcal{P}$ ) determined by $\epsilon$ (which, since $\mathcal{P}$ is connected, is easily seen to be independent of the choice of object of $\mathcal{P}-$ cf. Remark 1.1.1). We shall refer to as a $(\mathcal{P}$-)Frobenius-section of the Frobenioid $\mathcal{C}$ any homomorphism of monoids

$$
\mathcal{F}: \mathbb{N}_{\geq 1} \rightarrow \operatorname{End}(\mathcal{P} \hookrightarrow \mathcal{C})
$$

satisfying the following conditions: (a) the composite of $\mathcal{F}$ with the homomorphism $\operatorname{End}(\mathcal{P} \hookrightarrow \mathcal{C}) \rightarrow \mathbb{N}_{\geq 1}$ determined by considering the Frobenius degree is the identity on $\mathbb{N}_{\geq 1}$; (b) the endomorphisms of objects of $\mathcal{C}$ determined by an element of $\operatorname{End}(\mathcal{P} \hookrightarrow \mathcal{C})$ in the image of $\mathcal{F}$ are base-identity endomorphisms of Frobenius type. We shall refer to a Frobeniussection $\mathcal{F}$ which is regarded as being known only up to composition with automorphisms of the monoid $\mathbb{N}_{\geq 1}$ as a quasi-Frobenius-section. If $\mathcal{F}$ is a Frobenius-section, then we shall refer to the endomorphisms of $\mathcal{C}$ induced by elements of $\operatorname{End}(\mathcal{P} \hookrightarrow \mathcal{C})$ in the image of $\mathcal{F}$ as $\mathcal{F}$-distinguished.

(iii) We shall refer to a pair $(\mathcal{P}, \mathcal{F})$, where $\mathcal{P}$ is a base-section of $\mathcal{C}$, and $\mathcal{F}$ is a $\mathcal{P}$-Frobeniussection of $\mathcal{C}$, as a base-Frobenius pair of $\mathcal{C}$; when $\mathcal{F}$ is regarded as being known only up to composition with automorphisms of the monoid $\mathbb{N}_{\geq 1}$, we shall refer to such a pair as a quasibase-Frobenius pair. If the Frobenioid $\mathcal{C}$ admits a base-Frobenius pair (or, equivalently, a quasi-base-Frobenius pair), then we shall say that $\mathcal{C}$ is of pre-model type.

Remark 2.7.1. The notions of a 'base-section' and 'Frobenius-section' are intended to be a sort of 'category-theoretic translation' of the notion of a 'choice of trivialization of a trivial line bundle', which occurs naturally when $\mathcal{C}$ is a category of trivial line bundles (cf. Remark 5.6.1 and Examples 6.1 and 6.3 below).

Remark 2.7.2. Suppose that $\mathcal{C}$ is of isotropic type. Let $(\mathcal{P}, \mathcal{F})$ be a base-Frobenius pair of $\mathcal{C}$. Then the only arrows of $\mathcal{C}$ which are both $\mathcal{F}$ - and $\mathcal{P}$-distinguished (hence base-identity automorphisms - cf., e.g., the factorization of Definition 1.3(iv)(a)) are the identity arrows. Suppose further that the Frobenioid $\mathcal{C}$ is of base-trivial type and that the category $\mathcal{C}$ is a skeleton. Then every morphism $\phi$ of $\mathcal{C}$ admits a factorization

$$
\phi=\alpha \circ \beta \circ \gamma,
$$


where $\alpha$ is $\mathcal{P}$-distinguished, $\beta$ is a base-identity pre-step endomorphism and $\gamma$ is $\mathcal{F}$ distinguished. Moreover, this factorization is unique (in the strict sense - i.e., not up to isomorphisms, etc.). (Indeed, the existence and uniqueness of the factorization in question follow immediately from Definition 1.3(iv)(a), the definition of $\mathcal{P}$-, $\mathcal{F}$-distinguished, our assumptions concerning $\mathcal{C}$, the total epimorphicity of $\mathcal{C}$ and the isomorphism of functors appearing in the definition of a 'pull-back morphism' in Definition 1.2(ii).)

Definition 2.8.

(i) If, for every $A \in \mathrm{Ob}(\mathcal{C})$, it holds that $\mathcal{O}^{\times}(A)$ admits a (uniquely determined) profinite topology such that $\mathcal{O}^{\times}(A)$, equipped with this topology, is a topologically finitely generated profinite (abelian) group, then we shall say that $\mathcal{C}$ is of unit-profinite type.

(ii) Suppose that $M$ is a topologically finitely generated profinite abelian group. Thus, $M$ decomposes as a direct product of pro- $l$ groups $M[l]$, where $l$ varies over the elements of Primes (cf. Section 0). We shall refer to the factor $M[l]$ as the pro-l portion of $M$.

(iii) Let $M$ be as in (ii); assume that the group law of $M$ is written multiplicatively. If $\zeta: \mathfrak{P r i m e s} \rightarrow \mathbb{N}_{\geq 1}$ is a set-theoretic function, then we shall refer to the map $M \rightarrow M$

$$
(M \ni) a \mapsto a^{\zeta}(\in M)
$$

given by raising to the $\zeta(l)$ th power on $M[l]$, for $l \in \mathfrak{P r i m e s}$, as the map given by raising to the $\zeta$ th power on $M$. We shall refer to a set-theoretic function $\zeta: \mathfrak{P r i m e s} \rightarrow \mathbb{N}_{\geq 1}$ as being of co-prime type if $\zeta$ maps each element $l \in \mathfrak{P r i m e s}$ to an element of $\mathbb{N}_{\geq 1}$ that is prime to $l$. (Thus, if $\zeta$ is of co-prime type, then the map given by raising to the $\zeta$ th power will always be bijective.)

Proposition 2.9. (Unit-wise Frobenius functors II) Suppose that the Frobenioid $\mathcal{C}$ is of Frobenius-normalized, base-trivial, isotropic and Aut-ample (cf. Remark 2.9.2 below) type. Then we have the following.

(i) If the base category $\mathcal{D}$ admits a terminal object (cf. Section 0 ), then $\mathcal{C}$ is of pre-model type.

(ii) Let $\tau$ be a characteristic splitting on $\mathcal{C}$ and let $\zeta: \mathfrak{P r i m e s} \rightarrow \mathbb{N}_{\geq 1}$ be a set-theoretic function. Suppose that $\mathcal{C}$ is of pre-model and unit-profinite type. Then there exists a functor

$$
\Psi: \mathcal{C} \rightarrow \mathcal{C}
$$

which we shall refer to as the unit-wise Frobenius functor (associated to $\tau$, $\zeta$ ), which satisfies the following properties.

(a) $\Psi$ is 1-compatible, relative to the functor $\mathcal{C} \rightarrow \mathbb{F}_{\Phi}$, with the identity functor on $\mathbb{F}_{\Phi}$.

(b) $\Psi$ maps an object (respectively, morphism of Frobenius type; pre-step; pullback morphism) of $\mathcal{C}$ to an isomorphic object (respectively, abstractly equivalent morphism; abstractly equivalent morphism; abstractly equivalent morphism) of $\mathcal{C}$.

(c) If $A \in \mathrm{Ob}(\mathcal{C})$, then there exists an isomorphism $\Psi(A) \cong A$ such that the endomorphism of $\mathcal{O}^{\times}(A)$ induced by $\Psi$ followed by conjugation by this isomorphism is given by raising to the $\zeta$ th power.

(d) If $\zeta$ is of co-prime type (cf. Definition 2.8(iii)), then $\Psi$ is an equivalence of categories. If $\mathcal{C}$ is of unit-trivial type, then $\Psi$ is isomorphic to the identity functor. 
Proof. By well-known general nonsense in category theory, we may assume, without loss of generality, for the remainder of the proof of Proposition 2.9, that the category $\mathcal{C}$ is a skeleton. Thus, $\mathcal{C}^{\mathrm{pl}-\mathrm{bk}}$ is also a skeleton. Now we consider assertion (i). Observe (cf. Definition 1.3(i)(c) and the fact that $\mathcal{C}$ is of base-trivial type) that if $A \in \mathrm{Ob}(\mathcal{C})$ projects to a terminal object of $\mathcal{D}$, then $A$ is pseudo-terminal (cf. Section 0). Note that by Definition 1.3(i)(a), and our assumptions on $\mathcal{D}$, it follows that such an object $A$ always exists; let us fix one such object $A$. Thus, the natural projection functor determines an equivalence of categories

$$
\mathcal{C}_{A}^{\mathrm{pl-bk}} \stackrel{\sim}{\rightarrow} \mathcal{D}
$$

(cf. Definition 1.3(i)(c)). Note that it follows immediately from the existence of this equivalence of categories (together with the fact that $A$ maps to a terminal object of $\mathcal{D}$ ) that the natural functor $\mathcal{C}_{A}^{\mathrm{pl}-\mathrm{bk}} \rightarrow \mathcal{C}^{\mathrm{pl}-\mathrm{bk}}$ is injective on isomorphism classes of objects. In particular, if $\mathcal{Q} \subseteq \mathcal{C}_{A}^{\mathrm{pl}-\mathrm{bk}}$ is a skeletal subcategory (cf. Section 0), then (relative to some sufficiently large universe with respect to which, say, the category $\mathcal{C}$ is small) the natural map

$$
\mathrm{Ob}(\mathcal{Q}) \rightarrow \mathrm{Ob}\left(\mathcal{C}^{\mathrm{pl}-\mathrm{bk}}\right)=\mathrm{Ob}(\mathcal{C})
$$

is bijective (cf. the fact that $\mathcal{C}$ is of base-trivial type and the equivalence $\mathcal{C}_{A}^{\mathrm{pl}-\mathrm{bk}} \stackrel{\sim}{\rightarrow} \mathcal{D}$ ). Thus, the subcategory $\mathcal{P} \subseteq \mathcal{C}$ determined by the image of the objects and arrows of $\mathcal{Q}$ in $\mathcal{C}$ is a skeleton which satisfies the conditions of Definition 2.7(i) (cf. the fact that $\mathcal{C}$ is of base-trivial and isotropic type; Definition 1.3(i)(a)) - that is to say, $\mathcal{P}$ is a base-section.

Next, let us observe (cf. the fact that $\mathcal{C}$ is of base-trivial and isotropic type; Definition 1.3(i)(a)) that $A$ is Frobenius-trivial, hence that there exists a morphism of monoids

$$
\mathcal{F}_{A}: \mathbb{N}_{\geq 1} \rightarrow \operatorname{End}_{\mathcal{C}}(A)
$$

whose composite with the morphism of monoids $\operatorname{deg}_{\mathrm{Fr}}(-): \operatorname{End}_{\mathcal{C}}(A) \rightarrow \mathbb{N}_{\geq 1}$ is the identity morphism on $\mathbb{N}_{\geq 1}$, and whose image consists of base-identity endomorphisms of Frobenius type of $A$. Thus, by Proposition 1.11(iii), we conclude that $\mathcal{F}_{A}$ extends to a $\mathcal{P}$-Frobeniussection

$$
\mathcal{F}: \mathbb{N}_{\geq 1} \rightarrow \operatorname{End}(\mathcal{P} \rightarrow \mathcal{C})
$$

hence that $\mathcal{C}$ is of pre-model type, as desired. This completes the proof of assertion (i).

Next, we consider assertion (ii). Observe (cf. the fact that $\mathcal{C}$ is of base-trivial and isotropic type and the fact that $\mathcal{C}$ is a skeleton) we may apply Remark 2.7.2 to conclude that every morphism $\phi: C \rightarrow D$ of $\mathcal{C}$ admits a unique factorization

$$
\phi=\alpha \circ \beta \circ \gamma
$$

in $\mathcal{C}$, where $\alpha$ is $\mathcal{P}$-distinguished, $\beta$ is a base-identity pre-step endomorphism and $\gamma$ is $\mathcal{F}$-distinguished. Now (cf. the proof of Proposition 2.5(iii)) by applying the characteristic splitting (cf. Definition $2.3(\mathrm{a})) \mathcal{O}^{\times}(C) \times \tau(C) \stackrel{\sim}{\rightarrow} \mathcal{O}^{\triangleright}(C)$, we may write

$$
\beta=\beta_{0} \cdot \beta_{1} \in \mathcal{O}^{\triangleright}(C)
$$

(where $\beta_{0} \in \mathcal{O}^{\times}(C)$ and $\beta_{1} \in \tau(C)$ ). Set

$$
\Psi(\beta) \stackrel{\text { def }}{=} \beta_{0}^{\zeta} \cdot \beta_{1}, \quad \Psi(\phi) \stackrel{\text { def }}{=} \alpha \circ \Psi(\beta) \circ \gamma
$$


(where ' $(-)^{\zeta}$ ' is as defined in Definition 2.8(iii)). Since $\beta$ is completely determined by $\phi$, it follows that $\Psi$ is well-defined (as a 'map on arrows'). Moreover, it follows from the definition of $\mathcal{P}$ - and $\mathcal{F}$-distinguished morphisms (together with the fact that raising to the $\zeta$ th power defines an endomorphism of the functor in monoids ' $\mathrm{O}^{\times}(-)$' on $\mathcal{C}^{\text {lin }}$ which commutes with raising to the $d$ th power, for $d \in \mathbb{N}_{\geq 1}-\mathrm{cf}$. our assumption that $\mathcal{C}$ is of Frobenius-normalized type) that $\Psi$ is, in fact, a functor, and that $\Psi$ satisfies properties (a)-(d) in the statement of Proposition 2.9(ii). This completes the proof of Proposition 2.9.

Remark 2.9.1. By 'base-changing' the Frobenioid $\mathcal{C}$ via various functors $\mathcal{D}^{\prime} \rightarrow \mathcal{D}$ as in Proposition 1.6, it follows that one may obtain 'unit-wise Frobenius functors' as in Proposition 2.9(ii), for many Frobenioids whose base categories do not necessarily admit terminal objects (as is required in the hypotheses of Proposition 2.9(i)).

Remark 2.9.2. We shall see later (cf. Theorem 5.1(iii)) that in fact, the Aut-ampleness hypothesis in the statement of Proposition 2.9 is superfluous.

\section{Category-theoreticity of the base and Frobenius degree}

In this section, we show various results in the 'opposite direction' to the direction represented by the various Frobenius functors of Section 2. Namely, we show that various natural structures - such as Frobenius degrees and the natural projection functor to the base category - are preserved by equivalences of categories between Frobenioids.

In the following discussion, we maintain the notation of Sections 1 and 2. Also, we assume that we have been given a divisorial monoid $\Phi$ on a connected, totally epimorphic category $\mathcal{D}$ and a Frobenioid $\mathcal{C} \rightarrow \mathbb{F}_{\Phi}$.

Definition 3.1.

(i) We shall say that $\mathcal{C}$ is of quasi-isotropic type if it holds that $A \in \operatorname{Ob}(\mathcal{C})$ is non-isotropic if and only if it is an iso-subanchor (cf. Section 0). (Thus, if $\mathcal{C}$ is of isotropic type, then $\mathcal{C}$ is of quasi-isotropic type - cf. Remark 3.1.1 below.) We shall say that $\mathcal{C}$ is of standard type if the following conditions are satisfied:

(a) $\mathcal{C}$ is of quasi-isotropic and Frobenius-isotropic type;

(b) if $\mathcal{C}$ is of group-like type, then $\mathcal{C}^{\text {istr }}$ admits a Frobenius-compact object;

(c) $\mathcal{C}$ is of Frobenius-normalized type;

(d) $\mathcal{D}$ is of FSMFF-type;

(e) $\Phi$ is non-dilating.

We shall say that a category $\mathcal{E}$ is Frobenius-slim if every homomorphism of monoids

$$
\mathbb{F} \rightarrow \operatorname{Aut}\left(\mathcal{E}_{A} \rightarrow \mathcal{E}\right)
$$

(where $A \in \mathrm{Ob}(\mathcal{E})$ ) factors through the natural surjection $\mathbb{F} \rightarrow \mathbb{N}_{\geq 1}$. (Thus, every slim category is Frobenius-slim.)

(ii) Write $\mathcal{C}^{\text {Fr-tp }} \subseteq \mathcal{C}$ for the subcategory of $\mathcal{C}$ determined by the morphisms of Frobenius type and $\mathcal{C}^{\text {bi-Fr }} \subseteq \mathcal{C}^{\text {Fr-tp }} \times \mathcal{C}^{\text {Fr-tp }}$ for the subcategory of the product category $\mathcal{C}^{\text {Fr-tp }} \times \mathcal{C}^{\text {Fr-tp }}$ determined by pairs of morphisms of Frobenius type of the same Frobenius degree. 
For $A, B \in \mathrm{Ob}(\mathcal{C})$, we shall write

$$
\operatorname{Hom}_{\mathcal{C}}^{\mathrm{pf}}(A, B) \stackrel{\text { def }}{=} \underset{\left(A \rightarrow A^{\prime}, B \rightarrow B^{\prime}\right) \in \mathrm{Ob}\left({ }_{(A, B)} \mathcal{C}^{\text {bi-Fr }}\right)}{\lim _{\mathcal{C}}} \operatorname{Hom}_{\mathcal{C}}\left(A^{\prime}, B^{\prime}\right),
$$

where the inductive limit is parametrized by (say, some small skeletal subcategory of) ${ }_{(A, B)} \mathcal{C}^{\text {bi-Fr }}$ and the map

$$
\operatorname{Hom}_{\mathcal{C}}\left(A^{\prime}, B^{\prime}\right) \rightarrow \operatorname{Hom}_{\mathcal{C}}\left(A^{\prime \prime}, B^{\prime \prime}\right)
$$

induced by a morphism $\left(A^{\prime} \rightarrow A^{\prime \prime}, B^{\prime} \rightarrow B^{\prime \prime}\right)$ in ${ }_{(A, B)} \mathcal{C}^{\text {bi-Fr }}$ from an object $\left(A \rightarrow A^{\prime}, B \rightarrow\right.$ $\left.B^{\prime}\right)$ of ${ }_{(A, B)} \mathcal{C}^{\text {bi-Fr }}$ to an object $\left(A \rightarrow A^{\prime \prime}, B \rightarrow B^{\prime \prime}\right)$ of ${ }_{(A, B)} \mathcal{C}^{\text {bi-Fr }}$ is the map determined by the assignment ' $\phi \mapsto \phi^{\prime}$ ' of Proposition 1.10(i). We shall refer to an element of $\operatorname{Hom}_{\mathcal{C}}^{\mathrm{pf}}(A, B)$ as a perfected morphism $A \rightarrow B$.

(iii) Suppose that the Frobenioid $\mathcal{C}$ is of Frobenius-isotropic type. Then we shall write

$$
\mathcal{C}^{\text {pf }}
$$

for the category - which we shall refer to as the perfection of $\mathcal{C}$ - defined as follows: the objects of $\mathcal{C}^{\text {pf }}$ are pairs $(A, n)$, where $A \in \mathrm{Ob}(\mathcal{C}), n \in \mathbb{N}_{\geq 1}$. The morphisms of $\mathcal{C}^{\text {pf }}$ are given by

$$
\operatorname{Hom}_{\mathcal{C}^{\mathrm{pf}}}((A, n),(B, m)) \stackrel{\text { def }}{=} \operatorname{Hom}_{\mathcal{C}}^{\mathrm{pf}}\left(A^{\prime}, B^{\prime}\right),
$$

where $(A, n)$ and $(B, m)$ are objects of $\mathcal{C}^{\text {pf }}, A \rightarrow A^{\prime}$ is a morphism of Frobenius type in $\mathcal{C}$ of Frobenius degree $m, B \rightarrow B^{\prime}$ is a morphism of Frobenius type in $\mathcal{C}$ of Frobenius degree $n$, one verifies immediately (cf. Definition 1.3(ii)) that this set of morphisms of $\mathcal{C}^{\text {pf }}$ from $(A, n)$ to $(B, m)$ is independent (up to uniquely determined natural bijections) of the choice of morphisms of Frobenius type $A \rightarrow A^{\prime}, B \rightarrow B^{\prime}$ and composition of morphisms of $\mathcal{C}^{\text {pf }}$ is defined in the evident fashion. (Thus, in words, the pair $(A, n)$ is to be thought of as an ' $n t h$ root of $A^{\prime}$ '.) Also, we obtain a natural functor $\mathcal{C} \rightarrow \mathcal{C}^{\text {pf }}$ (by mapping ' $A \mapsto(A, 1)$ ').

(iv) Two co-objective (cf. Section 0) morphisms $\alpha_{1}, \alpha_{2}: A \rightarrow B$ of $\mathcal{C}^{\text {istr }}$ will be called unitequivalent if there exist morphisms $\gamma: A \rightarrow C$ and $\beta: C \rightarrow B$ (in $\mathcal{C}^{\text {istr }}$ ) and an automorphism $\delta \in \mathcal{O}^{\times}(C)$ such that $\alpha_{1}=\beta \circ \gamma$ and $\alpha_{2}=\beta \circ \delta \circ \gamma$. In this situation, we shall write $\alpha_{1} \stackrel{\mathcal{O}^{\times}}{\approx}$ $\alpha_{2}$. (Thus, if $\mathcal{C}$ is of unit-trivial type, then two co-objective morphisms of $\mathcal{C}^{\text {istr }}$ are unitequivalent if and only if they are equal.) By Proposition 3.3(ii) below, it follows that $\stackrel{\mathcal{O}^{\times}}{\approx}$, determines an equivalence relation on the set of morphisms $A \rightarrow B$ in $\mathcal{C}^{\text {istr }}$ which is, moreover, closed under composition of morphisms. We shall write

$$
\operatorname{Hom}_{\mathcal{C}^{\text {istr }}}^{\text {un-tr }}(A, B)
$$

for the set of unit-equivalence classes of morphisms $A \rightarrow B$. Also, we shall write

$$
\mathcal{C}^{\mathrm{un}-\mathrm{tr}}
$$

for the category whose objects are the objects of $\mathcal{C}^{\text {istr }}$, and whose morphisms are given by ' $\operatorname{Hom}_{\mathcal{C}}^{\text {un-trtr }}(-,-)$ ', and refer to $\mathcal{C}^{\text {un-tr }}$ as the unit-trivialization of $\mathcal{C}$. 
Remark 3.1.1. Observe that:

An iso-subanchor of the Frobenioid $\mathcal{C}$ is never isotropic. (In particular, if $\mathcal{C}$ is of isotropic type, then $\mathcal{C}$ is of quasi-isotropic type.)

Indeed, by Proposition 1.10(iv), an anchor is never isotropic. Thus, by Definition 1.3(vii)(b), a subanchor is never isotropic. Now if $B \rightarrow A$ is a mono-minimal categorical quotient (cf. Section 0) in $\mathcal{C}$ of $B$ by a group $G \subseteq \operatorname{Aut}_{\mathcal{C}}(B)$ such that $B$ is a subanchor and $A$ is isotropic, then applying the isotropification functor of Proposition 1.9(v) yields a factorization $B \rightarrow B^{\prime} \rightarrow A$, where $B \rightarrow B^{\prime}$ is an isotropic hull (hence a monomorphism cf. Definition 1.3(v)(a)), such that $G$ acts compatibly (relative to the arrow $B \rightarrow B^{\prime}$ ) on $B^{\prime}$; thus, by the definition of the term 'mono-minimal' it follows that the arrow $B \rightarrow B^{\prime}$ is an isomorphism, i.e., that $B$ is isotropic - a contradiction. This completes the proof of the 'observation'.

Remark 3.1.2. Observe that for any residually finite group $G$ (i.e., a group $G$ such that the intersection of the normal subgroups of finite index of $G$ is trivial):

Any homomorphism of monoids $\mathbb{F} \rightarrow G$ factors through the natural surjection $\mathbb{F} \rightarrow \mathbb{N}_{\geq 1}$.

(Indeed, it suffices to show this when $G$ is finite. When $G$ is finite, it follows immediately from the definition of $\mathbb{F}$ (cf. Definition 1.1(iii)) that the image of $1 \in \mathbb{Z}_{\geq 0}$ in $G$ is an element $\gamma \in G$ such that for every $d \in \mathbb{N}_{\geq 1}$, there exists an element $\delta_{d} \in G$ such that $\delta_{d} \cdot \gamma \cdot \delta_{d}^{-1}=\gamma^{d}$. Thus, by taking $d$ to be the order of $\gamma$, we conclude that $\gamma$ is the identity, hence that the homomorphism of monoids $\mathbb{F} \rightarrow G$ factors through the natural surjection $\mathbb{F} \rightarrow \mathbb{N}_{\geq 1}$, as desired.) In particular, it follows that if $\mathcal{E}$ is a category such that for every $A \in \operatorname{Ob}(\mathcal{E})$, the $\operatorname{group} \operatorname{Aut}\left(\mathcal{E}_{A} \rightarrow \mathcal{E}\right)$ is residually finite, then $\mathcal{E}$ is Frobenius-slim.

Remark 3.1.3. The phenomenon discussed in Remark 3.1.2 may be regarded as an example of the following fundamental dichotomy (which is, in a certain sense, a central theme of the theory of the present paper) between the structure of the base category $\mathcal{D}$ and the 'Frobenius structure' constituted by $\mathbb{N}_{\geq 1}$ :

\begin{tabular}{ll}
\hline Base category & Frobenius \\
\hline 'Indifferent to order' & 'Order-conscious' \\
Groups & Non-group-like monoids \\
\hline
\end{tabular}

This sort of phenomenon may be observed in 'classical scheme theory', for instance, in the invariance of the étale site of a scheme in positive characteristic under the Frobenius morphism. Here, it is useful to recall that a typical example of a 'base category' is constituted by the subcategory of connected objects of a Galois category (which is easily verified to be of $F S M$-, hence also of FSMFF-type). By contrast, categories such as $\operatorname{Order}\left(\mathbb{Z}_{\geq 0}\right), \operatorname{Order}\left(\mathbb{N}_{\geq 1}\right)$ or (the one-object categories determined by) $\mathbb{Z}_{\geq 0}, \mathbb{N}_{\geq 1}$ are not of FSMFF-type. In this context, it is interesting to note that categories such as $\operatorname{Order}(-)$ of a finite subset of $\mathbb{Z}_{\geq 0}$ of cardinality $\geq 2$ (with the induced ordering) constitute a sort of 'borderline case', which is of FSMFF-, but not of FSM-, type. 
Proposition 3.2. (Perfections of Frobenioids) Suppose that the Frobenioid $\mathcal{C}$ is of Frobenius-isotropic type. Then we have the following.

(i) There is a natural 1-commutative diagram of functors

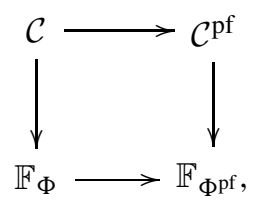

where the vertical arrow on the left is the functor that defines the Frobenioid structure on $\mathcal{C}$, the vertical arrow on the right is induced by the vertical arrow on the left, the lower horizontal arrow is induced by the natural morphism of monoids $\Phi \rightarrow \Phi^{\mathrm{pf}}$ and the upper horizontal arrow is the natural functor $\mathcal{C} \rightarrow \mathcal{C}^{\mathrm{pf}}$ of Definition 3.1(iii). In particular, the functor $\mathcal{C}^{\mathrm{pf}} \rightarrow \mathbb{F}_{\Phi \mathrm{pf}}$ determines a pre-Frobenioid structure on $\mathcal{C}^{\mathrm{pf}}$.

(ii) An arrow of $\mathcal{C}^{\mathrm{pf}}$ is $a(n)$ morphism of Frobenius type (respectively, pre-step; base-isomorphism; base-identity endomorphism; isomorphism; pull-back morphism; isometry; co-angular morphism; LB-invertible morphism; morphism of a given Frobenius degree) if and only if a cofinal collection of the system of arrows of $\mathcal{C}$ that determine this arrow of $\mathcal{C}^{\mathrm{pf}}$ (cf. Definition 3.1(ii)) is so.

(iii) The category $\mathcal{C}^{\mathrm{pf}}$, equipped with the functor $\mathcal{C}^{\mathrm{pf}} \rightarrow \mathbb{F}_{\Phi^{\mathrm{pf}}}$ of the diagram of (i), is a Frobenioid of perfect and isotropic type. Moreover, there is a natural equivalence of categories $\mathcal{C}^{\mathrm{pf}} \stackrel{\sim}{\rightarrow}\left(\mathcal{C}^{\mathrm{pf}}\right)^{\mathrm{pf}}$.

Proof. In light of our assumption that the Frobenioid $\mathcal{C}$ is of Frobenius-isotropic type, assertions (i)-(iii) follow immediately from the definitions and Proposition 1.10(i) (cf. also Proposition 2.1(iii)).

PROPOSITION 3.3. (Base-identity pre-steps and units)

(i) Write

$$
\operatorname{End}\left(\mathcal{C}_{A}^{\text {pl-bk }} \rightarrow \mathcal{C}\right)^{\text {bs-iso }} \subseteq \operatorname{End}\left(\mathcal{C}_{A}^{\text {pl-bk }} \rightarrow \mathcal{C}\right)
$$

(where $\mathcal{C}^{\mathrm{pl}-\mathrm{bk}}$ is as in Definition $1.3(i)(c)$ and $\mathcal{C}_{A}^{\mathrm{pl}-\mathrm{bk}} \rightarrow \mathcal{C}$ is the natural functor) for the submonoid consisting of those natural transformations such that the various endomorphisms of objects of $\mathcal{C}$ that occur in the natural transformation are all baseisomorphisms. Then if $\mathcal{D}$ is Frobenius-slim, then the image of $1 \in \mathbb{Z}_{\geq 0} \subseteq \mathbb{F}$ under any homomorphism of monoids

$$
\mathbb{F} \rightarrow \operatorname{End}\left(\mathcal{C}_{A}^{\mathrm{pl}-\mathrm{bk}} \rightarrow \mathcal{C}\right)^{\text {bs-iso }}
$$

determines an element of $\operatorname{End}\left(\mathcal{C}_{A}^{\mathrm{pl}-\mathrm{bk}} \rightarrow \mathcal{C}\right)^{\text {bs-iso }}$ with the property that the various endomorphisms of objects of $\mathcal{C}$ that occur in the natural transformation determined by this element are all base-identity pre-steps (i.e., lie in ' $\mathrm{O}^{\triangleright}(-)$ '). Conversely, if $\mathcal{C}$ is of Frobenius-normalized type, and $A$ is Frobenius-trivial, then every base-identity pre-step endomorphism of A arises as the endomorphism of A induced by the image of $1 \in \mathbb{Z}_{\geq 0} \subseteq \mathbb{F}$ via a homomorphism of monoids $\mathbb{F} \rightarrow \operatorname{End}\left(\mathcal{C}_{A}^{\mathrm{pl}-\mathrm{bk}} \rightarrow \mathcal{C}\right)^{\mathrm{bs}-\text { iso }}$.

(ii) Two co-objective morphisms $\alpha_{1}, \alpha_{2}: A \rightarrow B$ of $\mathcal{C}^{\text {istr }}$ are unit-equivalent if and only if they map to the same morphism of $\mathbb{F}_{\Phi}$, i.e., if and only if the following three conditions are satisfied: 
(a) $\operatorname{deg}_{\mathrm{Fr}}\left(\alpha_{1}\right)=\operatorname{deg}_{\mathrm{Fr}}\left(\alpha_{2}\right)$;

(b) $\operatorname{Div}\left(\alpha_{1}\right)=\operatorname{Div}\left(\alpha_{2}\right)$;

(c) $\operatorname{Base}\left(\alpha_{1}\right)=\operatorname{Base}\left(\alpha_{2}\right)$.

(iii) There is a natural functor

$$
\mathcal{C}^{\text {istr }} \rightarrow \mathcal{C}^{\text {un-tr }}
$$

which is full and essentially surjective; moreover, this functor is an equivalence of categories if and only if $\mathcal{C}^{\text {istr }}$ is of unit-trivial type.

(iv) The functor $\mathcal{C}^{\text {istr }} \rightarrow \mathbb{F}_{\Phi}$ factors naturally through $\mathcal{C}^{\text {un-tr }}$, hence determines a functor

$$
\mathcal{C}^{\text {un-tr }} \rightarrow \mathbb{F}_{\Phi}
$$

which is faithful and essentially surjective; moreover, this functor determines a natural structure of Frobenioid on $\mathcal{C}^{\text {un-tr }}$, with respect to which $\mathcal{C}^{\text {un-tr }}$ is of isotropic and unittrivial type. Finally, an arrow of $\mathcal{C}^{\text {un-tr }}$ is a $(n)$ morphism of Frobenius type (respectively, pre-step; base-isomorphism; isomorphism; pull-back morphism; isometry; co-angular morphism; LB-invertible morphism; morphism of a given Frobenius degree) if and only if it arises from such an arrow of $\mathcal{C}^{\text {istr. }}$.

(v) The functor

$$
\mathcal{C} \rightarrow \mathbb{F}_{\Phi}
$$

is an equivalence of categories if and only if the Frobenioid $\mathcal{C}$ is of Aut-ample, unittrivial and base-trivial type.

Proof. First, we consider assertion (i). Note that since the composite of the functor $\mathcal{C}_{A}^{\text {pl-bk }} \rightarrow \mathcal{C}$ with the natural projection functor $\mathcal{C} \rightarrow \mathcal{D}$ factors as the composite of the equivalence of

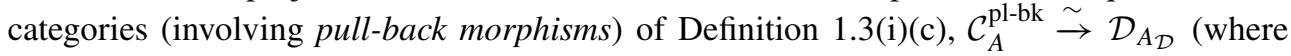
$A_{\mathcal{D}} \stackrel{\text { def }}{=} \operatorname{Base}(A)$ ) with the natural functor $\mathcal{D}_{A_{\mathcal{D}}} \rightarrow \mathcal{D}$, it follows that any homomorphism of monoids $\mathbb{F} \rightarrow \operatorname{End}\left(\mathcal{C}_{A}^{\mathrm{pl}-\mathrm{bk}} \rightarrow \mathcal{C}\right)^{\text {bs-iso }}$ determines a homomorphism of monoids

$$
\mathbb{F} \rightarrow \operatorname{Aut}\left(\mathcal{D}_{A_{\mathcal{D}}} \rightarrow \mathcal{D}\right),
$$

which, if $\mathcal{D}$ is Frobenius-slim (cf. Definition 3.1(i)), necessarily factors through the natural surjection $\mathbb{F} \rightarrow \mathbb{N}_{\geq 1}$, together with a homomorphism of monoids

$$
\mathbb{F} \rightarrow \mathbb{N}_{\geq 1}
$$

obtained by considering the Frobenius degree of the induced endomorphism of $A$, which (in light of the fact that the monoid $\mathbb{N}_{\geq 1}$ is commutative, together with the structure of $\mathbb{F}$ - cf. Definition 1.1(iii)) also necessarily factors through the natural surjection $\mathbb{F} \rightarrow \mathbb{N}_{\geq 1}$. Thus, we conclude that if $\mathcal{D}$ is Frobenius-slim, then the image of $1 \in \mathbb{Z}_{\geq 0} \subseteq \mathbb{F}$ under the given homomorphism of monoids $\mathbb{F} \rightarrow \operatorname{End}\left(\mathcal{C}_{A}^{\text {pl-bk }} \rightarrow \mathcal{C}\right)^{\text {bs-iso }}$ determines an element of $\operatorname{End}\left(\mathcal{C}_{A}^{\text {pl-bk }} \rightarrow \mathcal{C}\right)^{\text {bs-iso }}$ with the property that the various endomorphisms of objects of $\mathcal{C}$ that occur in the natural transformation determined by this element are all base-identity pre-steps, as desired.

The 'converse assertion' (when $\mathcal{C}$ is of Frobenius-normalized type, and $A$ is Frobeniustrivial) may be verified by choosing a homomorphism of monoids

$$
\mathbb{N}_{\geq 1} \rightarrow \operatorname{End}_{\mathcal{C}}(A)
$$


as in the definition of the term 'Frobenius-trivial' (cf. the homomorphism ' $\zeta$ ' of Definition 1.2(iv)), which, together with the homomorphism of monoids

$$
\mathbb{Z}_{\geq 0} \rightarrow \operatorname{End}_{\mathcal{C}}(A)
$$

that maps $1 \in \mathbb{Z}_{\geq 0}$ to a given base-identity pre-step endomorphism of $A$, yields (cf. our assumption that $\mathcal{C}$ is of Frobenius-normalized type!) a homomorphism of monoids

$$
\mathbb{F} \rightarrow \operatorname{End}_{\mathcal{C}}(A),
$$

which, by applying Proposition 1.11(iii), lifts to a homomorphism of monoids $\mathbb{F} \rightarrow$ $\operatorname{End}\left(\mathcal{C}_{A}^{\text {pl-bk }} \rightarrow \mathcal{C}\right)^{\text {bs-iso }}$, as desired. This completes the proof of assertion (i).

Next, we consider assertion (ii). Since assertion (ii) clearly only concerns the Frobenioid $\mathcal{C}^{\text {istr }}$ (cf. Proposition 1.9(v)), we may replace $\mathcal{C}$ by $\mathcal{C}^{\text {istr }}$ and assume for the remainder of the proof of assertion (ii) that $\mathcal{C}$ is of isotropic type. Now the necessity of the three conditions (a)-(c) follows immediately (cf. Remark 1.1.1) from the fact that endomorphisms of ' $\mathrm{O}^{\times}$, are LB-invertible base-identity linear endomorphisms. To show the sufficiency of these three conditions, we apply the factorization of Definition 1.3(iv)(a) (cf. also Proposition 1.4(i)), the essential uniqueness of morphisms of Frobenius type of a given Frobenius degree (cf. Definition 1.3(ii)), and the equivalence of categories (involving pull-back morphisms) of Definition 1.3(i)(c) to $\alpha_{1}$ and $\alpha_{2}$. Then conditions (a) and (c) imply that there exist morphisms $\gamma: A \rightarrow C, \beta_{1}, \beta_{2}: C \rightarrow D$ and $\delta: D \rightarrow B$, where $\gamma$ is a morphism of Frobenius type, $\beta_{1}$ and $\beta_{2}$ are base-equivalent co-angular pre-steps, and $\delta: D \rightarrow B$ is a pull-back morphism such that $\alpha_{1}=\delta \circ \beta_{1} \circ \gamma$ and $\alpha_{2}=\delta \circ \beta_{2} \circ \gamma$. Since $\delta$ and $\gamma$ are $L B$-invertible, it thus follows from condition (b) (cf. also Remark 1.1.1) that $\operatorname{Div}\left(\beta_{1}\right)=\operatorname{Div}\left(\beta_{2}\right)$, hence (by Definition 1.3(vi)) that $\beta_{2}=\zeta \circ \beta_{1}$, for some $\zeta \in \mathcal{O}^{\times}(D)$. Since $\alpha_{1}=\delta \circ\left(\beta_{1} \circ \gamma\right)$ and $\alpha_{2}=\delta \circ \zeta \circ\left(\beta_{1} \circ \gamma\right)$, we thus conclude that $\alpha_{1} \stackrel{\mathcal{O}^{\times}}{\approx} \alpha_{2}$, as desired. This completes the proof of assertion (ii). Now assertion (iii) is immediate from the definitions.

In light of assertions (ii) and (iii), assertion (iv) is immediate from the definitions. As for assertion (v), the necessity of the condition in the statement of assertion (v) follows immediately from Proposition 1.5(i) and (ii). To verify the sufficiency of this condition, let us first observe that if $\mathcal{C}$ is of unit-trivial and base-trivial type, then (by the existence of isotropic hulls in $\mathcal{C}-$ cf. Definition 1.3(vii)(a)) it follows that $\mathcal{C}$ is also of isotropic type, hence that we have a natural equivalence of categories $\mathcal{C} \stackrel{\sim}{\rightarrow} \mathcal{C}$ un-tr (cf. assertion (iii)). Thus, by assertion (iv), it follows that the natural functor $\mathcal{C} \rightarrow \mathbb{F}_{\Phi}$ is faithful and essentially surjective. Since $\mathcal{C}$ is of base-trivial and Aut-ample type, it follows from the factorization of Definition 1.3(iv)(a) (cf. also the existence and uniqueness of morphisms of Frobenius type of a given Frobenius degree asserted in Definition 1.3(ii) and the equivalence of categories involving pull-back morphisms of Definition 1.3(i)(c)), that to show that $\mathcal{C} \rightarrow \mathbb{F}_{\Phi}$ is full, it suffices to show that it is surjective on base-identity pre-step endomorphisms (i.e., on ' $\mathcal{O}^{\triangleright}(-)$ '); but, by our assumption that $\mathcal{C}$ is of base-trivial and Aut-ample type, this follows immediately from the first equivalence of categories of Definition 1.3(iii)(d). This completes the proof of assertion (v).

TheOREM 3.4. (Category-theoreticity of the base and Frobenius degree) For $i=1,2$, let $\Phi_{i}$ be a divisorial monoid on a connected, totally epimorphic category $\mathcal{D}_{i}$ let $\mathcal{C}_{i} \rightarrow \mathbb{F}_{\Phi_{i}}$ be a Frobenioid and let

$$
\Psi: \mathcal{C}_{1} \stackrel{\sim}{\rightarrow} \mathcal{C}_{2}
$$


be an equivalence of categories. Then we have the following.

(i) Suppose that $\mathcal{C}_{1}, \mathcal{C}_{2}$ are of quasi-isotropic type. Then $\Psi$ preserves the isotropic objects, isotropic hulls and isometric pre-steps. Moreover, there exists a 1-unique functor $\Psi^{\text {istr }}: \mathcal{C}_{1}^{\text {istr }} \rightarrow \mathcal{C}_{2}^{\text {istr }}$ that fits into a 1 -commutative diagram

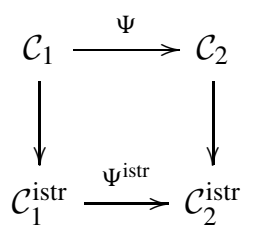

(where the vertical arrows are the natural 'isotropification functors' of Proposition $1.9(v)$ and the horizontal arrows are equivalences of categories). Finally, if $\mathcal{D}_{1}$, $\mathcal{D}_{2}$ are slim, and $\mathcal{C}_{1}, \mathcal{C}_{2}$ are of Frobenius-normalized type, then each of the composite functors of this diagram is rigid.

(ii) Suppose that $\mathcal{C}_{1}, \mathcal{C}_{2}$ are of quasi-isotropic type, and that $\mathcal{D}_{1}, \mathcal{D}_{2}$ are of FSMFF-type. Then $\Psi$ preserves pre-steps, co-angular pre-steps and group-like objects.

(iii) Suppose that: (a) $\mathcal{C}_{1}, \mathcal{C}_{2}$ are of standard type; (b) if $\mathcal{C}_{1}, \mathcal{C}_{2}$ are of group-like type, then both $\Psi$ and some quasi-inverse to $\Psi$ preserve base-isomorphisms. Then $\Psi$ preserves morphisms of Frobenius type, linear morphisms, base-isomorphisms, coangular morphisms, pull-back morphisms, isometries and LB-invertible morphisms. Moreover, there exists an automorphism of monoids

$$
\Psi^{\mathbb{N}_{\geq 1}}: \mathbb{N}_{\geq 1} \stackrel{\sim}{\rightarrow} \mathbb{N}_{\geq 1}
$$

such that $\Psi$ maps morphisms of Frobenius degree $d$ to morphisms of Frobenius degree $\Psi^{\mathbb{N}_{\geq 1}}(d)$; if $\mathcal{C}_{1}, \mathcal{C}_{2}$ admit a non-group-like object, then $\Psi_{\geq 1}^{\mathbb{N}}$ is the identity automorphism. Also, there exists a 1-unique functor $\Psi^{\mathrm{pf}}: \mathcal{C}_{1}^{\mathrm{pf}} \rightarrow \mathcal{C}_{2}^{\mathrm{pf}}$ that fits into a 1-commutative diagram

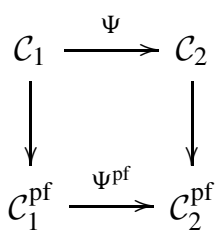

(where the vertical arrows are the natural functors of Proposition 3.2(i) and the horizontal arrows are equivalences of categories). Finally, if $\mathcal{D}_{1}, \mathcal{D}_{2}$ are slim, then each of the composite functors of this diagram is rigid.

(iv) Suppose that: (a) $\mathcal{C}_{1}, \mathcal{C}_{2}$ are of standard type; (b) if $\mathcal{C}_{1}, \mathcal{C}_{2}$ are of group-like type, then both $\Psi$ and some quasi-inverse to $\Psi$ preserve base-isomorphisms; (c) $\mathcal{D}_{1}, \mathcal{D}_{2}$ are Frobenius-slim. Then $\Psi$ preserves the submonoids ' $\mathcal{O}^{\triangleright}(-)$ ' and ' $\mathcal{O}^{\times}(-)$' and $\Psi^{\mathbb{N}_{\geq 1}}$ is the identity automorphism. Moreover, there exists a 1-unique functor $\Psi^{\mathrm{un}-\mathrm{tr}}: \mathcal{C}_{1}^{\mathrm{un}-\mathrm{tr}} \rightarrow$ 
$\mathcal{C}_{2}^{\text {un-tr }}$ that fits into a 1-commutative diagram

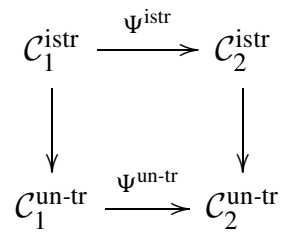

(where the vertical arrows are the natural functors of Proposition 3.3(iii) and the horizontal arrows are equivalences of categories). Finally, if $\mathcal{D}_{1}, \mathcal{D}_{2}$ are slim, then each of the composite functors of this diagram is rigid.

(v) Suppose that: (a) $\mathcal{C}_{1}, \mathcal{C}_{2}$ are of standard type; (b) if $\mathcal{C}_{1}, \mathcal{C}_{2}$ are of group-like type, then both $\Psi$ and some quasi-inverse to $\Psi$ preserve base-isomorphisms; (c) $\mathcal{D}_{1}, \mathcal{D}_{2}$ are slim. Then $\Psi$ preserves the base-identity endomorphisms and base-equivalent pairs of coobjective morphisms. Moreover, there exists a 1-unique functor $\Psi^{\text {Base }}: \mathcal{D}_{1} \rightarrow \mathcal{D}_{2}$ that fits into a 1-commutative diagram

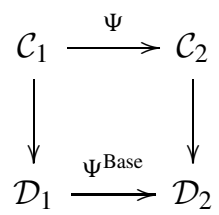

(where the vertical arrows are the natural projection functors and the horizontal arrows are equivalences of categories). Finally, each of the composite functors of this diagram is rigid.

Proof. First, we consider assertion (i). Since iso-subanchors are manifestly preserved by any equivalence of categories, it follows from our assumption that $\mathcal{C}_{1}, \mathcal{C}_{2}$ are of quasi-isotropic type and that $\Psi$ preserves isotropic objects. Now, with the exception of the final statement concerning the rigidity of the composite functors, the remainder of assertion (i) follows formally from (the definitions and) Proposition 1.9(v)-(vii). The final statement concerning the rigidity of the composite functors may be verified as follows. By Proposition 1.13(ii), it suffices to show for each $A \in \operatorname{Ob}\left(\mathcal{C}^{\text {istr }}\right)$ that the automorphism $\alpha \in \mathcal{O}^{\times}(A)$ induced by an automorphism $\in \operatorname{Aut}\left(\mathcal{C}_{1} \rightarrow \mathcal{C}_{1}^{\text {istr }}\right)$ is trivial. But, by Definition 1.3(i)(a), (b) and (iii)(c), it suffices to show this when $A$ is Frobenius-trivial, in which case the triviality of $\alpha$ follows from the functoriality of $\alpha$ with respect to base-identity endomorphisms of $A$ of arbitrary Frobenius degree (which implies, since $\mathcal{C}_{1}, \mathcal{C}_{2}$ are of Frobenius-normalized type, that $\alpha^{d}=\alpha$, for all $d \in \mathbb{N}_{\geq 1}$, hence that $\alpha$ is trivial, as desired).

Next, we consider assertion (ii). By assertion (i) (cf. also Proposition 1.9(v)), and the characterization of co-angular pre-steps given in Proposition 1.7(iv), we reduce immediately to the case where $\mathcal{C}_{1}, \mathcal{C}_{2}$ are of isotropic type. Then (since any equivalence of categories manifestly preserves FSM-morphisms and irreducible morphisms) the fact that $\Psi$ preserves pre-steps follows formally from Proposition 1.14(ii) and (iii). Since $\Psi$ preserves pre-steps, it thus follows from Proposition 1.8(iii) (cf. also Proposition 1.4(i)) that $\Psi$ preserves group-like objects. This completes the proof of assertions (i) and (ii). 
Next, we consider assertion (iii). First, I claim that to verify assertion (iii), it suffices to prove that, for each prime $p_{1} \in \mathfrak{P r i m e s}$, there exists a prime $p_{2} \in \mathfrak{P r i m e s}$, which is equal to $p_{1}$ if $\mathcal{C}_{1}, \mathcal{C}_{2}$ are not of group-like type, such that $\Psi^{\text {istr }}$ maps $p_{1}$-Frobenius morphisms to $p_{2}$-Frobenius morphisms. Indeed, the assignment $p_{1} \mapsto p_{2}$ determines a homomorphism of monoids

$$
\Psi^{\mathbb{N}_{\geq 1}}: \mathbb{N}_{\geq 1} \rightarrow \mathbb{N}_{\geq 1}
$$

which (by considering a quasi-inverse to $\Psi$ ) is easily seen to be an automorphism. Moreover, by Proposition 1.10(v), the condition of the claim implies that $\Psi^{\text {istr }}$ preserves morphisms of Frobenius type, hence also linear morphisms (by Proposition 1.7(iii)), and maps morphisms of Frobenius degree $d$ to morphisms of Frobenius degree $\Psi^{\mathbb{N}_{\geq 1}}(d)$ (i.e., since arbitrary morphisms may be written as composites of prime-Frobenius morphisms and linear morphisms - cf. Remark 1.1.1, Definition 1.3(iv)(a) and Proposition 1.10(v)). Since the isotropification functor preserves Frobenius degrees, this implies that $\Psi$ maps morphisms of Frobenius degree $d$ to morphisms of Frobenius degree $\Psi^{\mathbb{N}_{\geq 1}}(d)$, hence that $\Psi$ preserves linear morphisms and morphisms of Frobenius type (by Proposition 1.7(iii)). Moreover, by assertions (i) and (ii), $\Psi$ preserves isometric pre-steps and pre-steps, hence base-isomorphisms (i.e., composites of pre-steps and morphisms of Frobenius type - cf. Proposition 1.7(ii)), pull-back morphisms (cf. Proposition 1.7(ii)), isometries (i.e., morphisms that map via the isotropification functor to composites of a morphism of Frobenius type and a pull-back morphism - cf. Propositions 1.4(i), (v) and 1.9(v)), co-angular morphisms (cf. Definition 1.2(iii) and assertion (i) for isometric pre-steps) and LB-invertible morphisms. Now it follows immediately from the definition of $\mathcal{C}^{\text {pf }}$ (cf. Definition 3.1(iii)) that we obtain a 1-unique 1-commutative diagram as in the statement of assertion (iii). Finally, to verify the asserted rigidity of composite functors, it suffices (cf. the argument applied in the proof of assertion (i)) to apply Proposition 1.13(ii) and to consider the functoriality of the automorphisms in question with respect to base-identity endomorphisms of Frobenius-trivial objects of arbitrary Frobenius degree. This completes the proof of the claim.

Thus, to complete the proof of assertion (iii), we may assume (for the remainder of the proof of assertion (iii)) that $\mathcal{C}_{1}, \mathcal{C}_{2}$ are of isotropic type (cf. assertion (i)). Then it suffices to prove that, for each prime $p_{1} \in \mathfrak{P r i m e s}$, there exists a prime $p_{2} \in \mathfrak{P r i m e s}$, which is equal to $p_{1}$ if $\mathcal{C}_{1}, \mathcal{C}_{2}$ are not of group-like type, such that $\Psi$ maps $p_{1}$-Frobenius morphisms to $p_{2}$-Frobenius morphisms. Let us call an object $A_{1} \in \operatorname{Ob}\left(\mathcal{C}_{1}\right)\left(p_{1}, p_{2}\right)$-admissible if $\Psi$ maps every $p_{1}$-Frobenius morphism with domain $A_{1}$ to a $p_{2}$-Frobenius morphism of $\mathcal{C}_{2}$. Now let us consider the following assertions.

(F1) For each prime $p_{1} \in \mathfrak{P r i m e s}$, there exist a prime $p_{2} \in \mathfrak{P r i m e s}$ and a $\left(p_{1}, p_{2}\right)$ admissible object of $\mathcal{C}_{1}$.

(F2) For every pair of primes $p_{1}, p_{2} \in \mathfrak{P r i m e s}$ and every morphism $\zeta_{1}: A_{1} \rightarrow B_{1}$ in $\mathcal{C}_{1}, A_{1}$ is $\left(p_{1}, p_{2}\right)$-admissible if and only if $B_{1}$ is.

(F3) If $\mathcal{C}_{1}, \mathcal{C}_{2}$ are not of group-like type, then for each prime $p \in \mathfrak{P r i m e s}$, there exist a $(p, p)$ admissible object of $\mathcal{C}_{1}$.

Observe, moreover, that since $\mathcal{C}_{1}$ is connected, to complete the proof of assertion (iii), it suffices to prove (F1)-(F3).

First, we consider assertion (F1). Let us first consider the case where $\mathcal{C}_{1}, \mathcal{C}_{2}$ are of grouplike type. Then all pre-steps of $\mathcal{C}_{1}, \mathcal{C}_{2}$ are isomorphisms and $\Psi$ preserves base-isomorphisms. 
Thus, for any $A_{1} \in \operatorname{Ob}\left(\mathcal{C}_{1}\right)$, the prime-Frobenius morphisms with domain $A_{1}$ are precisely the irreducible base-isomorphisms with domain $A_{1}$ (cf. Proposition 1.14(i)). In particular, $\Psi$ preserves the prime-Frobenius morphisms; hence, we conclude that assertion (F1) holds. Next, let us consider the case where $\mathcal{C}_{1}, \mathcal{C}_{2}$ are not of group-like type. Then if $A_{1}$ is non-grouplike, then (cf. Definition 1.3(i)(a) and Proposition 1.8(iii)) there exists a base-isomorphic (i.e., to $A_{1}$ ), hence non-group-like, Frobenius-trivial object of $\mathcal{C}_{1}$. Thus, we may assume without loss of generality that $A_{1}$ is Frobenius-trivial. Then for any $p_{1} \in \mathfrak{P r i m e s}$, there exists a baseidentity (hence Div-identity) $p_{1}$-Frobenius endomorphism $\phi_{1}$ of $A_{1}$. Since (by assertion (ii)) $\Psi$ preserves pre-steps, it thus follows formally from the characterization of 'Div-identity prime-Frobenius endomorphisms' given in Proposition 1.14(v) that $\Psi$ maps $\phi_{1}$ to a primeFrobenius endomorphism of $A_{2} \stackrel{\text { def }}{=} \Psi\left(A_{1}\right)$. This completes the proof of assertion (F1).

Next, we consider assertion (F2). First, observe that if the morphism $\zeta_{1}: A_{1} \rightarrow B_{1}$ is a pre-step, then (since, by assertion (ii), $\Psi$ preserves pre-steps) it follows by applying Proposition 1.14(iv) to commutative diagrams such as the one given in Proposition 1.10(i), that assertion (F2) holds. Thus, by Definition 1.3(i)(a)-(c), we may assume without loss of generality that $B_{1}$ is Frobenius-trivial and that $\zeta_{1}$ is a pull-back morphism. Now, by applying Proposition 1.11(iii), it follows that for every $p_{1} \in \mathfrak{P r i m e s}$, there exist base-identity $p_{1}$ Frobenius endomorphisms $\phi_{1} \in \operatorname{End}_{\mathcal{C}}\left(A_{1}\right)$ and $\psi_{1} \in \operatorname{End}_{\mathcal{C}}\left(B_{1}\right)$ such that $\psi_{1} \circ \zeta_{1}=\zeta_{1} \circ \phi_{1}$. In particular, if we write $\phi_{2} \stackrel{\text { def }}{=} \Psi\left(\phi_{1}\right), \psi_{2} \stackrel{\text { def }}{=} \Psi\left(\psi_{1}\right)$ and $\zeta_{2} \stackrel{\text { def }}{=} \Psi\left(\zeta_{1}\right)$, then $\psi_{2} \circ \zeta_{2}=\zeta_{2} \circ \phi_{2}$ and $\phi_{2}, \psi_{2}$ are irreducible. Thus, by Proposition 1.14(iv) we obtain that $\phi_{2}$ is a $p_{2}$-Frobenius morphism if and only if $\psi_{2}$ is. This completes the proof of assertion (F2).

Finally, we consider assertion (F3). Let $A_{1} \in \mathrm{Ob}\left(\mathcal{C}_{1}\right)$ be a non-group-like, Frobeniustrivial object (cf. the proof of assertion (F1)). By assertions (F1) and (F2), it follows already that $\Psi$ preserves prime-Frobenius morphisms. Thus, to complete the proof of assertion (F3) (since the Frobenius degree of a prime-Frobenius morphism is always a prime number) it suffices to show that if $\zeta_{1}, \theta_{1} \in \operatorname{End}_{\mathcal{C}_{1}}\left(A_{1}\right)$ are prime-Frobenius base-identity endomorphisms such that $\operatorname{deg}_{\mathrm{Fr}}\left(\zeta_{1}\right)<\operatorname{deg}_{\mathrm{Fr}}\left(\theta_{1}\right)$, then $\operatorname{deg}_{\mathrm{Fr}}\left(\zeta_{2}\right)<\operatorname{deg}_{\mathrm{Fr}}\left(\theta_{2}\right)$ (where $\zeta_{2} \stackrel{\text { def }}{=}$ $\Psi\left(\zeta_{1}\right)$ and $\theta_{2} \stackrel{\text { def }}{=} \Psi\left(\theta_{1}\right)$ ). But, by the first equivalence of categories of Definition 1.3(iii)(d) (cf. also Proposition 1.10(i)), the condition ' $\operatorname{deg}_{\mathrm{Fr}}\left(\zeta_{1}\right)<\operatorname{deg}_{\mathrm{Fr}}\left(\theta_{1}\right)$ ' is equivalent to the following condition:

If we write $\beta_{\zeta_{1}}$ (respectively, $\beta_{\theta_{1}}$ ) for the step ' $\beta \circ \alpha$ ' of Proposition $1.14(\mathrm{v})$ obtained when one takes ' $\phi$ ' of loc. cit. to be $\zeta_{1}$ (respectively, $\theta_{1}$ ) (and ' $\alpha$ ' of loc. cit. to be some fixed step), then $\beta_{\theta_{1}}=\gamma_{1} \circ \beta_{\zeta_{1}}$ for some step $\gamma_{1}$.

Thus, if we write $\beta_{\zeta_{2}}$ (respectively, $\beta_{\theta_{2}}$ ) for the step ' $\beta \circ \alpha$ ' of Proposition 1.14(v), obtained when one takes ' $\phi$ ' of loc. cit. to be $\zeta_{2}$ (respectively, $\theta_{2}$ ) (and ' $\alpha$ ' of loc. cit. to be some fixed step), then $\beta_{\theta_{2}}=\gamma_{2} \circ \beta_{\zeta_{2}}$ for some step $\gamma_{2}$ (since, by assertion (ii), we already know that $\Psi$ preserves pre-steps), which (again by the first equivalence of categories of Definition 1.3(iii)(d) and Proposition 1.10(i)) implies that $\operatorname{deg}_{\mathrm{Fr}}\left(\zeta_{2}\right)<\operatorname{deg}_{\mathrm{Fr}}\left(\theta_{2}\right)$, as desired. This completes the proof of assertion (F3), hence also the proof of assertion (iii).

Next, let us observe that by assertion (i) (cf. also Proposition 1.9(v)), it suffices to verify assertions (iv) and (v) under the further assumption that $\mathcal{C}_{1}, \mathcal{C}_{2}$ are of isotropic type; thus, we assume for the remainder of the proof of Theorem 3.4 that $\mathcal{C}_{1}, \mathcal{C}_{2}$ are of isotropic type. 
Also, to simplify notation (for the remainder of the proof of Theorem 3.4), let us write

$$
\mathcal{P}_{i} \stackrel{\text { def }}{=} \mathcal{C}_{i}^{\mathrm{pl}-\mathrm{bk}}
$$

(cf. Definition 1.3(i)(c)), for $i=1,2$.

Next, let us consider assertion (iv). Now, for $i=1,2$, it follows formally (in light of our assumption that $\mathcal{D}_{i}$ is Frobenius-slim) from Proposition 3.3(i) (cf. also Definition 1.3(i)(a), (b) and (iii)(c)), that if $C \in \mathrm{Ob}\left(\mathcal{C}_{i}\right)$, then the endomorphisms of $\mathcal{O}^{\triangleright}(C)$ are precisely the endomorphisms $\gamma \in \operatorname{End}_{\mathcal{C}_{i}}(C)$ such that the following condition is satisfied:

There exist pre-steps $\phi: A \rightarrow B, \psi: A \rightarrow C$ and endomorphisms $\alpha \in \operatorname{End}_{\mathcal{C}_{i}}(A)$, $\beta \in \operatorname{End}_{\mathcal{C}_{i}}(B)$ such that $\beta \circ \phi=\phi \circ \alpha, \gamma \circ \psi=\psi \circ \alpha$ and, moreover, $\alpha$ arises as the endomorphism of $A$ induced by the image of $1 \in \mathbb{Z}_{\geq 0} \subseteq \mathbb{F}$ via a homomorphism of monoids $\mathbb{F} \rightarrow \operatorname{End}\left(\left(\mathcal{P}_{i}\right)_{A} \rightarrow \mathcal{C}_{i}\right)^{\text {bs-iso }}$.

By assertions (ii) and (iii), it follows that $\Psi$ preserves pre-steps, base-isomorphisms and pull-back morphisms, hence that $\Psi$ preserves endomorphisms satisfying the above condition. Thus, we conclude that $\Psi$ preserves the submonoids ' $\mathcal{O}^{\triangleright}(-)$ ' and ' $\mathcal{O}^{\times}(-)$', as desired. The existence of a 1-unique functor $\Psi^{\text {un-tr }}: \mathcal{C}_{1}^{\text {un-tr }} \rightarrow \mathcal{C}_{2}^{\text {un-tr }}$ that fits into a 1-commutative diagram as in the statement of assertion (iv) then follows formally from the definition of ' $\mathcal{C}_{1}^{\text {un-tr' }}$, ' $\mathcal{C}_{2}^{\text {un-tr'}}$ '; since ' $\mathcal{C}_{1}^{\text {un-tr }}$ ', ' $\mathcal{C}_{2}^{\text {un-tr' }}$ are of unit-trivial type, the asserted rigidity follows formally from Proposition 1.13(ii).

Thus, to complete the proof of assertion (iv), it suffices to show that $\Psi^{\mathbb{N}_{\geq 1}}$ is the identity automorphism. If $\mathcal{C}_{1}, \mathcal{C}_{2}$ are not of group-like type, then this already follows formally from assertion (iii). Thus, let us assume for the remainder of the proof of assertion (iv) that $\mathcal{C}_{1}, \mathcal{C}_{2}$ are of group-like type. Observe that there exists an object $A_{1} \in \operatorname{Ob}\left(\mathcal{C}_{1}\right)$ such that $A_{2} \stackrel{\text { def }}{=} \Psi\left(A_{1}\right)$ is Frobenius-compact (cf. Definition 3.1 and the fact that $\Psi$ is an equivalence of categories). By Proposition 1.10(vi), $A_{1}, A_{2}$ are Frobenius-trivial. Let $\phi_{1} \in \operatorname{End}_{\mathcal{C}_{1}}\left(A_{1}\right)$ be a base-identity prime-Frobenius endomorphism. By assertion (iii), $\phi_{2} \stackrel{\text { def }}{=} \Psi\left(\phi_{1}\right)$ is also a prime-Frobenius morphism. Write $\phi_{2}=\alpha \circ \psi_{2}$, where $\psi_{2}$ is a base-identity prime-Frobenius endomorphism of $A_{2}$ and $\alpha \in \operatorname{Aut}_{\mathcal{C}_{2}}\left(A_{2}\right)$ (cf. Definition 1.3(ii)). Now since $\mathcal{C}_{1}, \mathcal{C}_{2}$ are of Frobenius-normalized type (cf. Definition 3.1(i)(c)), it follows that for every $u_{1} \in \mathcal{O}^{\times}\left(A_{1}\right)$, $u_{1}^{p_{1}} \circ \phi_{1}=\phi_{1} \circ u_{1}$ (where $p_{1} \stackrel{\text { def }}{=} \operatorname{deg}_{\mathrm{Fr}}\left(\phi_{1}\right)$ ). Thus, for $u_{2} \in \mathcal{O}^{\times}\left(A_{2}\right)$, we obtain

$$
\begin{aligned}
u_{2}^{p_{1}} \circ \phi_{2} & =\phi_{2} \circ u_{2}=\alpha \circ \psi_{2} \circ u_{2}=\alpha \circ u_{2}^{p_{2}} \circ \psi_{2} \\
& =\alpha \circ u_{2}^{p_{2}} \circ \alpha^{-1} \circ \alpha \circ \psi_{2}=\alpha \circ u_{2}^{p_{2}} \circ \alpha^{-1} \circ \phi_{2}
\end{aligned}
$$

(where $p_{2} \stackrel{\text { def }}{=} \operatorname{deg}_{\mathrm{Fr}}\left(\phi_{2}\right)$ ), hence (by the total epimorphicity of $\mathcal{C}_{2}$ )

$$
u_{2}^{p_{1}}=\alpha \circ u_{2}^{p_{2}} \circ \alpha^{-1},
$$

i.e., $\alpha$ acts on $\mathcal{O}^{\times}\left(A_{2}\right)^{\text {pf }}$ by multiplication by $p_{1} / p_{2}$. Since $A_{2}$ is Frobenius-compact, we thus conclude that $p_{1}=p_{2}$. This completes the proof of assertion (iv).

Finally, we consider assertion (v). Now, for $i=1,2$, if $A \in \mathrm{Ob}\left(\mathcal{C}_{i}\right)=\mathrm{Ob}\left(\mathcal{P}_{i}\right)$ and $A_{\mathcal{D}} \stackrel{\text { def }}{=} \operatorname{Base}(A) \in \operatorname{Ob}\left(\mathcal{D}_{i}\right)$, then the natural projection functor $\mathcal{C}_{i} \rightarrow \mathcal{D}_{i}$ determines a natural equivalence of categories

$$
\left(\mathcal{P}_{i}\right)_{A} \stackrel{\sim}{\rightarrow}\left(\mathcal{D}_{i}\right)_{A_{\mathcal{D}}}
$$


(cf. Definition 1.3(i)(c)). Moreover, if $A^{\prime} \in \mathrm{Ob}\left(\mathcal{C}_{i}\right)=\mathrm{Ob}\left(\mathcal{P}_{i}\right)$ and $A_{\mathcal{D}}^{\prime} \stackrel{\text { def }}{=} \operatorname{Base}\left(A^{\prime}\right) \in \operatorname{Ob}\left(\mathcal{D}_{i}\right)$, then any arrow $A^{\prime} \rightarrow A$ determines a functor

$$
\left(\mathcal{P}_{i}\right)_{A^{\prime}} \rightarrow\left(\mathcal{P}_{i}\right)_{A}
$$

by sending an object $\phi: C^{\prime} \rightarrow A^{\prime}$ of $\left(\mathcal{P}_{i}\right)_{A^{\prime}}$ to the object $C \rightarrow A$ of $\left(\mathcal{P}_{i}\right)_{A}$ which is the pullback morphism of $\mathcal{C}_{i}$ that appears in the factorization of the composite $C^{\prime} \rightarrow A^{\prime} \rightarrow A$ given in Definition 1.3(iv)(a). Moreover, one verifies immediately that this functor fits into a natural 1-commutative diagram

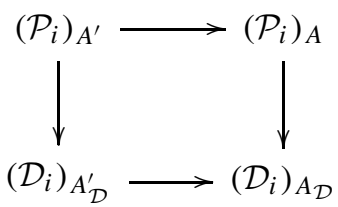

(where the upper horizontal arrow is the functor just defined, the vertical arrows are the equivalences that arise from the natural projection functor $\mathcal{C}_{i} \rightarrow \mathcal{D}_{i}$ and the lower horizontal arrow is the natural functor (cf. Section 0) determined by the arrow $A_{\mathcal{D}}^{\prime} \rightarrow A_{\mathcal{D}}$ obtained by projecting the given arrow $A^{\prime} \rightarrow A$ to $\mathcal{D}_{i}$ ).

Next, observe that since the category $\mathcal{D}_{i}$, hence also the categories $\left(\mathcal{D}_{i}\right)_{A_{\mathcal{D}}}$ and $\left(\mathcal{P}_{i}\right)_{A}$, are slim, it follows that the collection of categories ' $\left(\mathcal{P}_{i}\right)_{A}$ ' (where $i$ is fixed and $A$ ranges over the objects of $\mathcal{C}_{i}$ ) and functors ' $\left(\mathcal{P}_{i}\right)_{A^{\prime}} \rightarrow\left(\mathcal{P}_{i}\right)_{A}$ ' (arising from arrows $A^{\prime} \rightarrow A$ of $\mathcal{C}_{i}$ ) determine a 2-slim (cf. Definition A.1(i)) 2-category of 1-categories, whose 'coarsification' (cf. Definition A.1(ii)) we denote by $\mathcal{Q}_{i}$, together with a natural functor

$$
\mathcal{C}_{i} \rightarrow \mathcal{Q}_{i}
$$

(i.e., which maps $\left.A \mapsto\left(\mathcal{P}_{i}\right)_{A},\left(A^{\prime} \rightarrow A\right) \mapsto\left\{\left(\mathcal{P}_{i}\right)_{A^{\prime}} \rightarrow\left(\mathcal{P}_{i}\right)_{A}\right\}\right)$. Similarly, the collection of categories ' $\left(\mathcal{D}_{i}\right)_{A_{\mathcal{D}}}$ ' (where $i$ is fixed and $A_{\mathcal{D}}$ ranges over the objects of $\mathcal{D}_{i}$ ) and functors ' $\left(\mathcal{D}_{i}\right)_{A_{\mathcal{D}}^{\prime}} \rightarrow\left(\mathcal{D}_{i}\right)_{A_{\mathcal{D}}}$ ' (arising from arrows $A_{\mathcal{D}}^{\prime} \rightarrow A_{\mathcal{D}}$ of $\mathcal{D}_{i}$ ) determine a 2-category of 1-categories, whose coarsification we denote by $\mathcal{E}_{i}$, together with a natural functor

$$
\mathcal{D}_{i} \rightarrow \mathcal{E}_{i},
$$

which, in fact, may be identified with the 'slim exponentiation functor' of Proposition A.2, hence, in particular, is an equivalence of categories. Thus, since the natural projection functor $\mathcal{C}_{i} \rightarrow \mathcal{D}_{i}$ is essentially surjective (cf. Definition 1.3(i)(a)), it follows that the natural projection functor $\mathcal{C}_{i} \rightarrow \mathcal{D}_{i}$ induces a faithful, essentially surjective functor

$$
\mathcal{Q}_{i} \rightarrow \mathcal{E}_{i}
$$

which may be composed with a quasi-inverse to the natural equivalence $\mathcal{D}_{i} \stackrel{\sim}{\rightarrow} \mathcal{E}_{i}$ just discussed to obtain a faithful, essentially surjective functor

$$
\mathcal{Q}_{i} \rightarrow \mathcal{D}_{i}
$$

(which is well-defined up to isomorphism).

Next, let us observe that if $A, A^{\prime} \in \operatorname{Ob}\left(\mathcal{C}_{i}\right), A_{\mathcal{D}} \stackrel{\text { def }}{=} \operatorname{Base}(A)$ and $A_{\mathcal{D}}^{\prime} \stackrel{\text { def }}{=} \operatorname{Base}\left(A^{\prime}\right)$, then any morphism $\phi_{\mathcal{D}}: A_{\mathcal{D}} \rightarrow A_{\mathcal{D}}^{\prime}$ may be written in the form

$$
\phi_{\mathcal{D}}=\operatorname{Base}(\psi) \circ \operatorname{Base}(\gamma) \circ \operatorname{Base}(\alpha)^{-1},
$$


where $\alpha: B \rightarrow A$ and $\gamma: B \rightarrow C$ are pre-steps and $\psi: C \rightarrow A^{\prime}$ is a pull-back morphism (cf. Definition 1.3(i)(b), (c)). Since (by the above discussion) any base-isomorphism $\zeta: D \rightarrow E$ of $\mathcal{C}_{i}$ induces an equivalence of categories $\left(\mathcal{P}_{i}\right)_{D_{\mathcal{D}}} \stackrel{\sim}{\rightarrow}\left(\mathcal{P}_{i}\right)_{E_{\mathcal{D}}}$ (where $D, E \in \operatorname{Ob}\left(\mathcal{C}_{i}\right)$, $D_{\mathcal{D}} \stackrel{\text { def }}{=} \operatorname{Base}(D)$ and $\left.E_{\mathcal{D}} \stackrel{\text { def }}{=} \operatorname{Base}(E)\right)$, it thus follows that any collection of morphisms $\alpha$, $\gamma$ and $\psi$ as just described determine a 'new functor'

$$
\left(\mathcal{P}_{i}\right)_{A_{\mathcal{D}}} \rightarrow\left(\mathcal{P}_{i}\right)_{A_{\mathcal{D}}^{\prime}}
$$

(i.e., by inverting the equivalence of categories induced by $\alpha$ and then composing with the functors induced by $\gamma$ and $\psi$ ). Thus, by enlarging the 2-slim 2-category of 1-categories considered above (i.e., whose coarsification we called $\mathcal{Q}_{i}$ ) by considering these "new functors', we obtain a (slightly larger) 2-slim 2-category of 1-categories, whose coarsification we denote by $\mathcal{R}_{i}$. In particular, we obtain a (faithful) embedding $\mathcal{Q}_{i} \hookrightarrow \mathcal{R}_{i}$ with the property that the functor $\mathcal{Q}_{i} \rightarrow \mathcal{D}_{i}$ considered above admits a natural extension to a functor

$$
\mathcal{R}_{i} \rightarrow \mathcal{D}_{i}
$$

which (by the above discussion) is clearly an equivalence of categories.

On the other hand, since (by assertions (ii) and (iii)) $\Psi$ preserves pre-steps, pullback morphisms and factorizations as in Definition 1.3(iv)(a), it follows that $\Psi$ induces a 1-commutative diagram

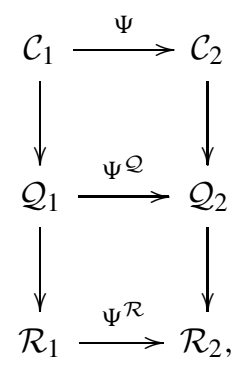

where the vertical functors are the natural functors of the above discussion and the horizontal functors are equivalences of categories induced by $\Psi$. Thus, by composing with the natural equivalences of categories $\mathcal{R}_{i} \stackrel{\sim}{\rightarrow} \mathcal{D}_{i}$ of the above discussion, we obtain a 1-commutative diagram as in the statement of assertion (v), which is clearly 1-unique (cf. Definition 1.3(i)(a)-(c)). Finally, the asserted rigidity follows formally from Proposition 1.13(i). This completes the proof of assertion (v).

Remark 3.4.1. With regard to assumption (b) of Theorem 3.4(iii), (iv) and (v), we observe the following. Suppose, in the situation of Theorem 3.4, that $\mathcal{C}_{1}, \mathcal{C}_{2}$ are of group-like and quasiisotropic type. Then if $\Psi$ and some quasi-inverse to $\Psi$ preserve Frobenius degrees, then they also preserve base-isomorphisms. Indeed, by Theorem 3.4(i) we may assume, without loss of generality, that $\mathcal{C}_{1}, \mathcal{C}_{2}$ are of isotropic type. Then if $\Psi$ and some quasi-inverse to $\Psi$ preserve Frobenius degrees, then they preserve linear morphisms, hence morphisms of Frobenius type (cf. Proposition 1.7(iii)) and base-isomorphisms (i.e., morphisms of Frobenius type, since $\mathcal{C}_{1}$, $\mathcal{C}_{2}$ are of group-like and isotropic type - cf. Propositions 1.4(i), 1.7(ii) and 1.8(iii)).

One way to understand the meaning of the conditions imposed in the various portions of Theorem 3.4 is by considering examples in which some of the conditions hold, but others do not. 
Example 3.5. (Base categories with FSMI-endomorphisms) Let $\mathcal{D}$ be a one-object category whose unique object has endomorphism monoid $\mathbb{F}$ and $\mathcal{C}$ a one-object category whose unique object has endomorphism monoid $\mathbb{F} \times \mathbb{F}$. Thus, the projection $\mathbb{F} \times \mathbb{F} \rightarrow \mathbb{F}$ to the first factor determines a functor $\mathcal{C} \rightarrow \mathcal{D}$ and $\mathcal{C}$ may be identified with the elementary Frobenioid determined by the (manifestly non-dilating) monoid on $\mathcal{D}$ that assigns to the unique object of $\mathcal{D}$ the monoid $\mathbb{Z}_{\geq 0}$ and to every morphism of $\mathcal{D}$ the identity automorphism of $\mathbb{Z}_{\geq 0}$. In particular, $\mathcal{C}$ is a Frobenioid of Frobenius-normalized and isotropic type, which is not of group-like type (cf. Proposition 1.5(i), (ii)). On the other hand, one verifies immediately that every morphism of $\mathcal{D}$ is an FSM-morphism and that the endomorphism $1 \in \mathbb{Z}_{\geq 0} \subseteq \mathbb{F}$ of the unique object of $\mathcal{D}$ is irreducible. Thus, $\mathcal{D}$ admits an FSMI-endomorphism, which implies (cf. Section 0 ) that $\mathcal{D}$ fails to be of FSMFF-type. Moreover, the self-equivalence of $\mathcal{C}$ determined by the automorphism of monoids

$$
\mathbb{F} \times \mathbb{F} \stackrel{\sim}{\rightarrow} \mathbb{F} \times \mathbb{F}
$$

given by switching the two factors clearly fails to preserve pre-steps (cf. Theorem 3.4(ii)).

Example 3.6. (Frobenioids of standard and group-like type) Let

$$
G \stackrel{\text { def }}{=} \mathbb{Z} \oplus\left(\bigoplus_{p \in \mathfrak{P r i m e s}} \mathbb{Z} / p \mathbb{Z}\right)
$$

(regarded as an abelian group), let $\mathcal{D}$ be a one-object category whose unique object has endomorphism monoid $\mathbb{F}_{G}$, and let $\mathcal{C}$ be a one-object category whose unique object has endomorphism monoid $\mathbb{F}_{G} \times \mathbb{F}_{G}$. Thus, if $A \in \operatorname{Ob}(\mathcal{D})$, then each automorphism of an object of $\mathcal{D}$ arising from an element $\operatorname{Aut}\left(\mathcal{D}_{A} \rightarrow \mathcal{D}\right)$ is contained in the subgroup of infinitely divisible elements of $G$ (cf. the proof of Proposition 1.13(iii)), hence is trivial - that is to say, $\mathcal{D}$ is slim. Moreover, the projection $\mathbb{F}_{G} \times \mathbb{F}_{G} \rightarrow \mathbb{F}_{G}$ to the first factor determines a functor $\mathcal{C} \rightarrow \mathcal{D}$ and $\mathcal{C}$ may be identified with the elementary Frobenioid determined by the (manifestly non-dilating) monoid on $\mathcal{D}$ that assigns to the unique object of $\mathcal{D}$ the monoid $G$ and to every morphism of $\mathcal{D}$ the identity automorphism of $G$. In particular, $\mathcal{C}$ is a Frobenioid of Frobenius-normalized, isotropic, and group-like type (cf. Proposition 1.5(i), (iii)). One verifies immediately that every morphism of $\mathcal{D}$ is either an isomorphism or a nonmonomorphism (cf. the existence of the torsion subgroup $\bigoplus_{p \in \mathfrak{P r i m e s}} \mathbb{Z} / p \mathbb{Z} \subseteq G$ ), and that the irreducible morphisms of $\mathcal{D}$ are precisely the morphisms that project via the natural surjection $\mathbb{F}_{G} \rightarrow \mathbb{N}_{\geq 1}$ to primes of $\mathbb{N}_{\geq 1}$. Thus, it follows immediately that $\mathcal{D}$ is of FSM-, hence also of FSMFF-type. Moreover, since $G^{\mathrm{pf}} \cong \mathbb{Q} \neq 0$, and the first factor of $\mathbb{F}_{G}$ in the product $\mathbb{F}_{G} \times \mathbb{F}_{G}$ commutes with the $G$ (i.e., ' $\mathcal{O}^{\times}(-)$') of the second factor of $\mathbb{F}_{G}$, it follows that the unique object of $\mathcal{C}$ is Frobenius-compact. Thus, $\mathcal{C}$ is of standard type. On the other hand, the self-equivalence of $\mathcal{C}$ determined by the automorphism of monoids

$$
\mathbb{F}_{G} \times \mathbb{F}_{G} \stackrel{\sim}{\rightarrow} \mathbb{F}_{G} \times \mathbb{F}_{G}
$$

given by switching the two factors clearly fails to preserve base-isomorphisms (cf. Theorem 3.4(iii)).

Example 3.7. (Dilating monoids) Let $G, \mathcal{D}$ be as in Example 3.6 and let $\Phi$ be the monoid on $\mathcal{D}$ that associates to the unique object of $\mathcal{D}$ the monoid $G \times \mathbb{Z}_{\geq 0}$ and to a morphism $f \in \mathbb{F}_{G}$ of 
$\mathcal{D}$ that projects to an element $d_{f} \in \mathbb{N}_{\geq 1}$ the endomorphism of $G \times \mathbb{Z}_{\geq 0}$ that acts trivially on $G$ and by multiplication by $d_{f}$ on $\mathbb{Z}_{\geq 0}$. Thus (as observed in Example 3.6), D is of FSMFF-type, but $\Phi$ clearly fails to be non-dilating. Write $\mathcal{C} \stackrel{\text { def }}{=} \mathbb{F}_{\Phi}$. Thus, $\mathcal{C}$ is a Frobenioid of Frobeniusnormalized and isotropic type, which is not of group-like type (cf. Proposition 1.5(i), (ii)). Moreover, $\mathcal{C}$ is a one-object category whose unique object has endomorphism monoid $M$ given by the product set

$$
\mathbb{Z}_{\geq 0} \times\left(\mathbb{F}_{G} \times \mathbb{F}_{G}\right)
$$

equipped with the following monoid structure: if $a_{1}, a_{2} \in \mathbb{Z}_{\geq 0}$ and $b_{1}, b_{2} \in \mathbb{F}_{G} \times \mathbb{F}_{G}$, where $b_{1}$ projects to an element $(n, m) \in \mathbb{N}_{\geq 1} \times \mathbb{N}_{\geq 1}$, then

$$
\left(a_{1}, b_{1}\right) \cdot\left(a_{2}, b_{2}\right)=\left(a_{1}+n \cdot m \cdot a_{2}, b_{1} \cdot b_{2}\right)
$$

(cf. the description of elementary Frobenioids in Definition 1.1(iii)). Thus, by switching the two factors of $\mathbb{F}_{G}$, and keeping the unique factor of $\mathbb{Z}_{\geq 0}$ fixed, we obtain an automorphism of the monoid $M$, hence a self-equivalence of $\mathcal{C}$, that preserves pre-steps (cf. Theorem 3.4(ii)), but fails to preserve base-isomorphisms (cf. Theorem 3.4(iii)).

Example 3.8. (Permutation of primes) Let $\alpha: \mathbb{N}_{\geq 1} \stackrel{\sim}{\rightarrow} \mathbb{N}_{\geq 1}$ be an automorphism of monoids of order $2, N \stackrel{\text { def }}{=}\left(\mathbb{N}_{\geq 1}\right)^{\text {gp }}$ (so $\alpha$ acts on $N$ ), $U \stackrel{\text { def }}{=} \mathbb{Q}, V \stackrel{\text { def }}{=} \mathbb{Q}, W \stackrel{\text { def }}{=} \mathbb{Q}, G \stackrel{\text { def }}{=} U \rtimes N$, where we let $n \in N(\subseteq \mathbb{Q})$ act on $U$ by $n^{-1}, \mathcal{D}$ the one-object category whose unique object has endomorphism monoid $G, \Phi$ the (manifestly non-dilating) monoid on $\mathcal{D}$ that associates to the unique object of $\mathcal{D}$ the monoid $V \times W$ and to a morphism $g \in G$ that projects to an element $n \in N$ the automorphism of $V \times W$ given by $\left(\alpha(n), \alpha(n) \cdot n^{-1}\right)$ (i.e., the automorphism that acts on $V$ by $\alpha(n)$ and on $W$ by $\left.\alpha(n) \cdot n^{-1}\right)$ and $\mathcal{C} \stackrel{\text { def }}{=} \mathbb{F}_{\Phi}$. Thus, $\mathcal{C}$ is a Frobenioid of Frobenius-normalized, isotropic and group-like type (cf. Proposition 1.5(i), (iii)) and the category $\mathcal{D}$ is manifestly of FSM-, hence also of FSMFF-type (cf. Section 0 ). Since the unique object of $\mathcal{C}$ has ' $\mathcal{O}^{\times}(-)$' equal to $V \times W$, it follows from our definition of $\Phi$ that this object is Frobenius-compact. Thus, $\mathcal{C}$ is of standard type. On the other hand, if $A \in \mathrm{Ob}(\mathcal{D})$, then $\operatorname{Aut}\left(\mathcal{D}_{A} \rightarrow \mathcal{D}\right) \cong G$; since there exist injections of monoids $\mathbb{F} \hookrightarrow G$, it thus follows that $\mathcal{D}$ fails to be Frobenius-slim. The monoid $M$ of endomorphisms of the unique object of $\mathcal{C}$ may be described as the product set

$$
U \times V \times W \times N \times \mathbb{N}_{\geq 1}
$$

equipped with the following monoid structure: if $u_{1}, u_{2} \in U, v_{1}, v_{2} \in V, w_{1}, w_{2} \in W$, $n_{1}, n_{2} \in N$ and $m_{1}, m_{2} \in \mathbb{N}_{\geq 1}$, then

$$
\begin{aligned}
& \left(u_{1}, v_{1}, w_{1}, n_{1}, m_{1}\right) \cdot\left(u_{2}, v_{2}, w_{2}, n_{2}, m_{2}\right) \\
& \quad=\left(u_{1}+n_{1}^{-1} \cdot u_{2}, v_{1}+m_{1} \cdot \alpha\left(n_{1}\right) \cdot v_{2}, w_{1}+m_{1} \cdot \alpha\left(n_{1}\right) \cdot n_{1}^{-1} \cdot w_{2}, n_{1} \cdot n_{2}, m_{1} \cdot m_{2}\right)
\end{aligned}
$$

(cf. the description of elementary Frobenioids in Definition 1.1(iii)). In particular, a routine verification reveals that the assignment

$$
(u, v, w, n, m) \mapsto\left(v, u, w, \alpha(n)^{-1} \cdot m^{-1}, \alpha(m)\right)
$$

(where $u \in U, v \in V, w \in W, n \in N$ and $m \in \mathbb{N}_{\geq 1}$ ) determines an automorphism of the monoid $M$, hence a self-equivalence of $\mathcal{C}$, which clearly preserves base-isomorphisms, but fails to preserve ' $\mathcal{O}^{\times}(-)$' (i.e., the subspace $\{0\} \times V \times W \subseteq U \times V \times W$ ) or Frobenius degrees (when $\alpha$ is not equal to the identity) - cf. Theorem 3.4(iii), (iv). 
Example 3.9. (Non-preservation of units) Let $N \stackrel{\text { def }}{=}\left(\mathbb{N}_{\geq 1}\right)^{\text {gp }}, U \stackrel{\text { def }}{=} \mathbb{Q}, V \stackrel{\text { def }}{=} \mathbb{Q}, W \stackrel{\text { def }}{=} \mathbb{Z}_{\geq 0}$, $G \stackrel{\text { def }}{=} U \rtimes N$, where we let $n \in N(\subseteq \mathbb{Q})$ act on $U$ by $n^{-1}, \mathcal{D}$ the one-object category whose unique object has endomorphism monoid $G, \Phi$ the (manifestly non-dilating) monoid on $\mathcal{D}$ that associates to the unique object of $\mathcal{D}$ the monoid $V \times W$ and to a morphism $g \in G$ that projects to an element $n \in N$ the automorphism of $V \times W$ given by $(n, 1)$ (i.e., the automorphism that acts on $V$ by $n$ and on $W$ by 1 ) and $\mathcal{C} \stackrel{\text { def }}{=} \mathbb{F}_{\Phi}$. Thus, $\mathcal{C}$ is a Frobenioid of Frobenius-normalized and isotropic type, which is not of group-like type (cf. Proposition 1.5(i), (ii)) and $\mathcal{D}$ is manifestly of FSM-, hence also of FSMFF-type (cf. Section 0 ). Thus, $\mathcal{C}$ is of standard type. On the other hand (cf. Example 3.8), $\mathcal{D}$ fails to be Frobenius-slim. The monoid $M$ of endomorphisms of the unique object of $\mathcal{C}$ may be described as the product set

$$
U \times V \times W \times N \times \mathbb{N}_{\geq 1}
$$

equipped with the following monoid structure: if $u_{1}, u_{2} \in U, v_{1}, v_{2} \in V, w_{1}, w_{2} \in W$, $n_{1}, n_{2} \in N$ and $m_{1}, m_{2} \in \mathbb{N}_{\geq 1}$, then

$$
\begin{aligned}
& \left(u_{1}, v_{1}, w_{1}, n_{1}, m_{1}\right) \cdot\left(u_{2}, v_{2}, w_{2}, n_{2}, m_{2}\right) \\
& \quad=\left(u_{1}+n_{1}^{-1} \cdot u_{2}, v_{1}+m_{1} \cdot n_{1} \cdot v_{2}, w_{1}+m_{1} \cdot w_{2}, n_{1} \cdot n_{2}, m_{1} \cdot m_{2}\right)
\end{aligned}
$$

(cf. the description of elementary Frobenioids in Definition 1.1(iii)). In particular, a routine verification reveals that the assignment

$$
(u, v, w, n, m) \mapsto\left(v, u, w, n^{-1} \cdot m^{-1}, m\right)
$$

(where $u \in U, v \in V, w \in W, n \in N$ and $m \in \mathbb{N}_{\geq 1}$ ) determines an automorphism of the monoid $M$, hence a self-equivalence of $\mathcal{C}$, which clearly fails to preserve ' $\mathcal{O}^{\times}(-)$', ' $\mathcal{O}^{\triangleright}(-)$ ' (i.e., the subspaces $\{0\} \times V \times\{0\}$ and $\{0\} \times V \times W \subseteq U \times V \times W)-$ cf. Theorem 3.4(iv).

Example 3.10. (Non-slim base categories) Let $G$ be a group, whose center we denote by $Z(G)$, let $\mathcal{D}$ be a one-object category whose unique object has endomorphism monoid $G$ and let $\mathcal{C}$ be a one-object category whose unique object has endomorphism monoid $G \times \mathbb{F}$. Thus, the projection $G \times \mathbb{F} \rightarrow G$ determines a functor $\mathcal{C} \rightarrow \mathcal{D}$ where $\mathcal{C}$ may be identified with the elementary Frobenioid determined by the (manifestly non-dilating) monoid on $\mathcal{D}$ that assigns to the unique object of $\mathcal{D}$ the monoid $\mathbb{Z}_{\geq 0}$ and to every morphism of $\mathcal{D}$ the identity automorphism of $\mathbb{Z}_{\geq 0}$. In particular, $\mathcal{C}$ is a Frobenioid of Frobenius-normalized and isotropic type, which is not of group-like type (cf. Proposition 1.5(i), (ii)) and $\mathcal{D}$ is manifestly of FSM-, hence also of FSMFF-type (cf. Section 0 ). Thus, $\mathcal{C}$ is of standard type. On the other hand, if $\alpha: \mathbb{F} \rightarrow Z(G)$ is any non-trivial homomorphism of monoids that factors as the composite of the natural surjection $\mathbb{F} \rightarrow \mathbb{N}_{\geq 1}$ with a homomorphism of monoids $\mathbb{N}_{\geq 1} \rightarrow Z(G)$, then the automorphism of monoids

$$
\begin{gathered}
G \times \mathbb{F} \stackrel{\sim}{\rightarrow} G \times \mathbb{F} \\
(g, f) \mapsto(g \cdot \alpha(f), f)
\end{gathered}
$$

(where $g \in G, f \in \mathbb{F}$ ) determines a self-equivalence $\mathcal{C} \stackrel{\sim}{\rightarrow} \mathcal{C}$ which clearly fails to preserve base-identity endomorphisms of Frobenius type (cf. Theorem 3.4(v)). 
Finally, before proceeding, we consider the case of Frobenioids of group-like type in a bit more detail.

PROPOSITION 3.11. (Frobenioids of isotropic, unit-trivial and group-like type) For $i=1,2$, let $\Phi_{i}$ be the zero monoid (more precisely: any functor $\mathcal{D}_{i} \rightarrow \mathfrak{M o n}$ all of whose values are monoids of cardinality one) on a connected, totally epimorphic category $\mathcal{D}_{i}$ of FSMFF-type let $\mathcal{C}_{i} \rightarrow \mathbb{F}_{\Phi_{i}}$ be a Frobenioid of isotropic, unit-trivial and group-like type and let

$$
\Psi: \mathcal{C}_{1} \stackrel{\sim}{\rightarrow} \mathcal{C}_{2}
$$

be an equivalence of categories. Then we have the following.

(i) The functor $\mathcal{C}_{i} \rightarrow \mathbb{F}_{\Phi_{i}}$ is an equivalence of categories.

(ii) $\Psi$ preserves base-isomorphisms, pull-back morphisms, linear morphisms and morphisms of Frobenius type.

(iii) Suppose that both $\Psi$ and some quasi-inverse to $\Psi$ preserve base-identity endomorphisms. Then there exists a 1-unique functor $\Psi^{\text {Base }}: \mathcal{D}_{1} \rightarrow \mathcal{D}_{2}$ that fits into a 1-commutative diagram

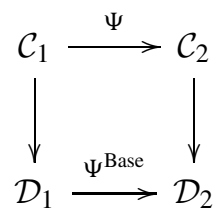

(where the vertical arrows are the natural projection functors and the horizontal arrows are equivalences of categories). Finally, if $\mathcal{D}_{1}, \mathcal{D}_{2}$ are slim, then each of the composite functors of this diagram is rigid.

Proof. First, we consider assertion (i). By Proposition 3.3(iii) and (iv), the functor $\mathcal{C}_{i} \rightarrow \mathbb{F}_{\Phi_{i}}$ is essentially surjective and faithful. Since the Frobenioid $\mathcal{C}_{i}$ is of grouplike and isotropic type, it follows that every pre-step of $\mathcal{C}_{i}$ is an isomorphism (cf. Propositions 1.4(i) and 1.8(iii)), hence that the Frobenioid $\mathcal{C}_{i}$ is of Aut-ample and base-trivial (cf. Definition 1.3(i)(b)), as well as unit-trivial, type. Thus, it follows from Proposition 3.3(v) that the functor $\mathcal{C}_{i} \rightarrow \mathbb{F}_{\Phi_{i}}$ is an equivalence of categories. This completes the proof of assertion (i).

Next, we consider assertion (ii). Observe that since $\mathcal{D}_{i}$ is of FSMFF-type, it follows that $\mathcal{D}_{i}$ has no FSMI-endomorphisms (cf. Section 0), hence that a morphism of $\mathcal{C}_{i}$ is an FSMI-endomorphism if and only if it is a prime-Frobenius endomorphism (cf. Propositions 1.11(vi) and 1.14(i) and the evident structure of $\mathbb{F}_{\Phi_{i}}$ ). Thus, $\Psi$ preserves the prime-Frobenius endomorphisms, hence also prime-Frobenius morphisms (since every prime-Frobenius morphism is abstractly equivalent to a prime-Frobenius endomorphism). But this implies that $\Psi$ preserves the morphisms of Frobenius type (cf. Proposition 1.10(v)), hence also the linear morphisms (cf. Proposition 1.7(iii)). Since the (co-angular) presteps of $\mathcal{C}_{i}$ are isomorphisms (cf. Proposition 1.8(iii)), it thus follows that $\Psi$ preserves the pull-back morphisms (cf. Proposition 1.7(iii)), as well as the base-isomorphisms (cf. Proposition 1.7(ii)). This completes the proof of assertion (ii).

Finally, we consider assertion (iii). Write $\mathcal{N}$ for the one-object category whose unique object has endomorphism monoid equal to $\mathbb{N}_{\geq 1}$. Then we have equivalences of categories

$$
\mathcal{C}_{i} \stackrel{\sim}{\rightarrow} \mathbb{F}_{\Phi_{i}} \stackrel{\sim}{\rightarrow} \mathcal{D}_{i} \times \mathcal{N}
$$


(cf. assertion (i)). Moreover, one verifies immediately that the base-identity endomorphisms of $\mathcal{C}_{i}$ are precisely the endomorphisms of $\mathcal{C}_{i} \stackrel{\sim}{\rightarrow} \mathcal{D}_{i} \times \mathcal{N}$ that arise from elements of $\mathbb{N}_{\geq 1}$; let us refer to such endomorphisms as ' $\mathbb{N}_{\geq 1}$-endomorphisms'. Thus, it follows from our assumption concerning the preservation of base-identity endomorphisms that the $\mathbb{N}_{\geq 1}$ endomorphisms are preserved by $\Psi$. Note, moreover, that $\mathcal{D}_{i}$ may be reconstructed from $\mathcal{C}_{i}$ by considering equivalence classes of morphisms of $\mathcal{C}_{i}$, where two morphisms of $\mathcal{C}_{i}$ are regarded as equivalent if they admit composites with an $\mathbb{N}_{\geq 1}$-endomorphism which are equal. Thus, we obtain a 1-commutative diagram as in the statement of assertion (ii). Finally, the rigidity assertion in the statement of assertion (ii) follows immediately from Proposition 1.13(i).

\section{Category-theoreticity of the divisor monoid}

In this section, we show that the monoid on the base category that appears in the definition of a Frobenioid (cf. Definition 1.3) may, under suitable conditions, be reconstructed entirely category-theoretically. Together with the results of Section 3, this allows us to conclude, under suitable conditions, that the functor to an elementary Frobenioid that appears in the definition of a Frobenioid (cf. Definition 1.3) may be recovered entirely from the structure of a Frobenioid as an abstract category (cf. Corollary 4.11).

In the following discussion, we maintain the notation of Sections 1-3. Also, we assume that we have been given a divisorial monoid $\Phi$ on a connected, totally epimorphic category $\mathcal{D}$ and a Frobenioid $\mathcal{C} \rightarrow \mathbb{F}_{\Phi}$.

Proposition 4.1. (Primary steps) Suppose further that $\mathcal{C}$ is of perfect and isotropic type, and that $\Phi$ is perf-factorial. Let $A \in \mathrm{Ob}(\mathcal{C})$ be Div-Frobenius-trivial and let

$$
\phi: B \rightarrow A, \quad \psi: A \rightarrow C, \quad \delta: D \rightarrow E, \quad \epsilon: E \rightarrow F, \quad \iota: I \rightarrow F
$$

be steps of $\mathcal{C}$. For $n \in \mathbb{N}_{\geq 1}$, let $\alpha_{n} \in \operatorname{End}_{\mathcal{C}}(A)$ be a Div-identity endomorphism of Frobenius type such that $\operatorname{deg}_{\mathrm{Fr}}\left(\alpha_{n}\right)=n$. Then we have the following.

(i) $\phi$ is primary if and only if, for every factorization $\phi=\phi_{A} \circ \phi_{B}$, where $\phi_{B}: B \rightarrow B^{\prime}$ and $\phi_{A}: B^{\prime} \rightarrow A$ are steps, there exists a commutative diagram

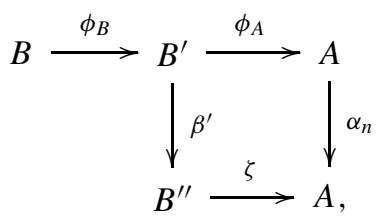

where $n \in \mathbb{N}_{\geq 1}, \beta^{\prime}$ is a morphism of Frobenius type and $\zeta=\phi \circ \zeta^{\prime}$ and $\zeta^{\prime}: B^{\prime \prime} \rightarrow B$ are pre-steps.

(ii) Suppose that $\phi$ is primary. Then the composite $\psi \circ \phi: B \rightarrow C$, hence also $\psi$, is primary if and only if, for every factorization $\psi \circ \phi=\psi^{\prime} \circ \phi^{\prime}$, where $\phi^{\prime}: B \rightarrow A^{\prime}$ and $\psi^{\prime}: A^{\prime} \rightarrow C$ are steps, there exist factorizations $\phi=\zeta \circ \phi^{\prime \prime}$ and $\phi^{\prime}=\zeta^{\prime} \circ \phi^{\prime \prime}$, where $\phi^{\prime \prime}: B \rightarrow A^{\prime \prime}$ is a step and $\zeta: A^{\prime \prime} \rightarrow A$ and $\zeta^{\prime}: A^{\prime \prime} \rightarrow A^{\prime}$ are pre-steps.

(iii) $\epsilon_{*}(\operatorname{Div}(\epsilon)), \iota_{*}(\operatorname{Div}(\iota)) \in \Phi(F)$ (where we write $\epsilon_{*}, \iota_{*}$ for the respective bijections induced by the functor $\Phi$ ) have disjoint supports ( $f$. Definition $2.4(i)(d))$ if and only if every pre-step $\zeta: Z \rightarrow F$ such that there exist pre-steps $\epsilon^{\prime}, \iota^{\prime}$ satisfying $\epsilon=\zeta \circ \epsilon^{\prime}$, 
$\iota=\zeta \circ \iota^{\prime}$ is, in fact, an isomorphism. In this case, we shall say that $\epsilon, \iota$ are co-primary. If $\epsilon, \iota$ are co-primary, then there exists a cartesian diagram in the category of pre-steps

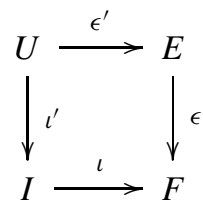

such that $\epsilon_{*}\left(\epsilon_{*}^{\prime}\left(\operatorname{Div}\left(\epsilon^{\prime}\right)\right)\right)=\iota_{*}(\operatorname{Div}(\iota)), \iota_{*}\left(\iota_{*}^{\prime}\left(\operatorname{Div}\left(\iota^{\prime}\right)\right)\right)=\epsilon_{*}(\operatorname{Div}(\epsilon))$; if $\epsilon, \iota$ are primary, then so are $\epsilon^{\prime}, \iota^{\prime}$.

(iv) $\delta$ is primary if and only if there exists $a \mathfrak{p} \in \operatorname{Prime}(\Phi(F))$ such that the following condition holds: for every primary $\epsilon^{\prime}: E^{\prime} \rightarrow F$ such that $\epsilon_{*}^{\prime}\left(\operatorname{Div}\left(\epsilon^{\prime}\right)\right) \notin \mathfrak{p}$ (where we write $\epsilon_{*}^{\prime}$ for the bijection induced by the functor $\left.\Phi\right)$, there exists a factorization $\epsilon=\epsilon^{\prime} \circ \zeta$, where $\zeta$ is a pre-step, if and only if there exists a factorization $\epsilon \circ \delta=\epsilon^{\prime} \circ \theta$, where $\theta$ is a pre-step.

(v) $\epsilon$ is primary if and only if there exists $a \mathfrak{p} \in \operatorname{Prime}(\Phi(D))$ such that the following condition holds: for every primary $\delta^{\prime}: D \rightarrow E^{\prime}$ such that $\operatorname{Div}\left(\delta^{\prime}\right) \notin \mathfrak{p}$, there exists a factorization $\delta=\zeta \circ \delta^{\prime}$, where $\zeta$ is a pre-step, if and only if there exists a factorization $\epsilon \circ \delta=\theta \circ \delta^{\prime}$, where $\theta$ is a pre-step.

Proof. First, we consider assertion (i). By applying the second equivalence of categories of Definition 1.3(iii)(d) to the various pre-steps over $A$, it follows that, if we write $x_{\phi} \stackrel{\text { def }}{=}$ $\phi_{*}(\operatorname{Div}(\phi)) \in \Phi(A)$ (where we write $\phi_{*}$ for the bijection induced by the functor $\left.\Phi\right)$, then the condition of assertion (i) may be translated into the language of monoids as follows:

For every equation $x_{\phi}=x_{A}+x_{B}$ in $\Phi(A)$, where $x_{A}, x_{B} \neq 0$, we have $x_{\phi} \preccurlyeq x_{A}$.

Now the equivalence of this condition with the condition that $x_{\phi}$ is primary follows immediately from the definition of the term 'primary' (cf. Section 0), together with the fact that $\Phi(A)$ is perfect (cf. Proposition 1.10(iii)). This completes the proof of assertion (i).

Next, we consider assertion (ii). Again, we apply Definition 1.3(iii)(d) to the various pre-steps over $C$, to obtain the following translation of the condition of assertion (ii) into the language of monoids (where we set $\left.x_{\phi} \stackrel{\text { def }}{=} \psi_{*}\left(\phi_{*}(\operatorname{Div}(\phi))\right), x_{\psi} \stackrel{\text { def }}{=} \psi_{*}(\operatorname{Div}(\psi)) \in \Phi(C)\right)$ :

For every equation $x_{\phi}+x_{\psi}=x_{\phi^{\prime}}+x_{\psi^{\prime}}$ in $\Phi(A)$, where $x_{\phi^{\prime}}, x_{\psi^{\prime}} \neq 0$, there exists a $0 \neq x_{\phi^{\prime \prime}} \in \Phi(A)$ such that $x_{\phi^{\prime \prime}} \leq x_{\phi}, x_{\phi^{\prime \prime}} \leq x_{\phi^{\prime}}$.

Now the necessity of this condition follows immediately from the structure of the $\Phi(A)_{\mathfrak{p}}$, where $\mathfrak{p} \in \operatorname{Prime}(\Phi(A)$ ) (cf. Definition 2.4(i)(b)), whereas the sufficiency of this condition follows by taking $x_{\phi^{\prime}} \leq x_{\psi}$ (cf. Definition 2.4(i)(c), (d) and the fact that $\Phi(A)$ is perfect). This completes the proof of assertion (ii).

Next, we consider assertion (iii). By applying the second equivalence of categories of Definition 1.3(iii)(d) to the various pre-steps over $F$, we obtain the following translation of the condition of assertion (iii) into the language of monoids (where we set $x_{\epsilon} \stackrel{\text { def }}{=}$ $\left.\epsilon_{*}(\operatorname{Div}(\epsilon)), x_{\iota} \stackrel{\text { def }}{=} \iota_{*}(\operatorname{Div}(\iota)) \in \Phi(F)\right)$ :

Every $x_{\zeta} \in \Phi(F)$ such that $x_{\zeta} \leq x_{\epsilon}, x_{\zeta} \leq x_{\iota}$ is, in fact, equal to 0 . 
The necessity and sufficiency of this condition then follow immediately by considering the 'primary factorizations' of $x_{\epsilon}, x_{\iota}$ (cf. Definition 2.4(i)(c), (d) and the fact that $\Phi(A)$ is perfect). The cartesian diagram (with the desired properties) then follows from the fact that 'for $x_{U} \in \Phi(F), x_{\epsilon}+x_{\iota} \leq x_{U}$ if and only if $x_{\epsilon} \leq x_{U}, x_{\iota} \leq x_{U}$ ' (cf. Definition 2.4(i)(c), (d) and the fact that $\Phi(A)$ is perfect). This completes the proof of assertion (iii).

Next, we consider assertion (iv). This time, we apply the second equivalence of categories of Definition 1.3(iii)(d) to the various pre-steps over $F$, to obtain the following translation of the condition of assertion (iv) concerning $\mathfrak{p} \in \operatorname{Prime}(\Phi(F))$ into the language of monoids (where we set $x_{\delta} \stackrel{\text { def }}{=} \epsilon_{*}\left(\delta_{*}((\operatorname{Div}(\delta))), x_{\epsilon} \stackrel{\text { def }}{=} \epsilon_{*}(\operatorname{Div}(\epsilon)) \in \Phi(F)\right)$ :

For every primary element $x_{\epsilon^{\prime}} \notin \mathfrak{p}, x_{\epsilon^{\prime}} \leq x_{\epsilon}$ if and only if $x_{\epsilon^{\prime}} \leq x_{\delta}+x_{\epsilon}$.

The necessity and sufficiency of this condition then follow immediately by comparing the 'primary factorizations' of $x_{\epsilon}, x_{\delta}+x_{\epsilon}$ (cf. Definition 2.4(i)(c), (d) and the fact that $\Phi(A)$ is perfect). Also, we observe that assertion (v) follows by an entirely similar argument obtained by 'reversing the direction of the arrows'. This completes the proof of assertions (iv) and (v).

THEOREM 4.2. (Category-theoreticity of primary steps) For $i=1,2$, let $\Phi_{i}$ be a perffactorial divisorial monoid on a connected, totally epimorphic category $\mathcal{D}_{i}$ let $\mathcal{C}_{i} \rightarrow \mathbb{F}_{\Phi_{i}}$ be $a$ Frobenioid of standard and isotropic type, which is not of group-like type and let

$$
\Psi: \mathcal{C}_{1} \stackrel{\sim}{\rightarrow} \mathcal{C}_{2}
$$

be an equivalence of categories. Then we have the following.

(i) $\Psi$ preserves primary steps, Div-identity endomorphisms, Div-Frobenius-trivial objects and universally Div-Frobenius-trivial objects.

(ii) There exists a unique isomorphism $\Psi^{\text {Prime }}$ between the functors

$$
\mathrm{Ob}\left(\mathcal{C}_{i}^{\text {bs-iso }}\right) \ni A_{i} \mapsto \operatorname{Prime}\left(\Phi_{i}\left(A_{i}\right)\right)
$$

(where $i=1,2)$ on $\mathcal{C}_{i}^{\text {bs-iso }}$ which satisfies the following property. Suppose that $A_{2}=$ $\Psi\left(A_{1}\right)$ and $\mathfrak{p}_{1} \in \operatorname{Prime}\left(\Phi_{1}\left(A_{1}\right)\right), \mathfrak{p}_{2} \in \operatorname{Prime}\left(\Phi_{2}\left(A_{2}\right)\right)$ correspond under $\Psi^{\text {Prime }}$. For $i=1,2$, write

$$
\left\{A_{i}\left(\mathcal{C}_{i}^{\text {coa-pre }}\right)\right\}_{\mathfrak{p}_{i}} \stackrel{\sim}{\rightarrow} \operatorname{Order}\left(\Phi_{i}\left(A_{i}\right)_{\mathfrak{p}_{i}}\right), \quad\left\{\left(\mathcal{C}_{i}^{\text {coa-pre }}\right)_{A_{i}}\right\}_{\mathfrak{p}_{i}} \stackrel{\sim}{\rightarrow} \operatorname{Order}\left(\Phi_{i}\left(A_{i}\right)_{\mathfrak{p}_{i}}\right)^{\text {opp }}
$$

for the respective full subcategories and restricted equivalences of categories determined by the full subcategory

$$
\operatorname{Order}\left(\Phi_{i}\left(A_{i}\right)_{\mathfrak{p}_{i}}\right) \subseteq \operatorname{Order}\left(\Phi_{i}\left(A_{i}\right)\right)
$$

arising from the submonoid $\Phi_{i}\left(A_{i}\right)_{\mathfrak{p}_{i}} \subseteq \Phi_{i}\left(A_{i}\right)$. Then the map induced by $\Psi$ on presteps (cf. (i) and Theorem 3.4(ii)) induces equivalences of categories

$$
\left\{A_{1}\left(\mathcal{C}_{1}^{\text {coa-pre }}\right)\right\}_{\mathfrak{p}_{1}} \stackrel{\sim}{\rightarrow}\left\{A_{A_{2}}\left(\mathcal{C}_{2}^{\text {coa-pre }}\right)\right\}_{\mathfrak{p}_{2}}, \quad\left\{\left(\mathcal{C}_{1}^{\text {coa-pre }}\right)_{A_{1}}\right\}_{\mathfrak{p}_{1}} \stackrel{\sim}{\rightarrow}\left\{\left(\mathcal{C}_{2}^{\text {coa-pre }}\right)_{A_{2}}\right\}_{\mathfrak{p}_{2}}
$$

hence equivalences of categories as

$$
\begin{aligned}
& \operatorname{Order}\left(\Phi_{1}\left(A_{1}\right)_{\mathfrak{p}_{1}}\right) \stackrel{\sim}{\rightarrow} \operatorname{Order}\left(\Phi_{2}\left(A_{2}\right)_{\mathfrak{p}_{2}}\right), \\
& \operatorname{Order}\left(\Phi_{1}\left(A_{1}\right)_{\mathfrak{p}_{1}}\right)^{\text {opp }} \stackrel{\sim}{\rightarrow} \operatorname{Order}\left(\Phi_{2}\left(A_{2}\right)_{\mathfrak{p}_{2}}\right)^{\text {opp }}
\end{aligned}
$$


(iii) If, moreover, in the situation of (ii), the $A_{i}$ are Div-Frobenius-trivial, then the last two equivalences of categories of (ii) arise from isomorphisms of monoids

$$
\Phi_{1}\left(A_{1}\right)_{\mathfrak{p}_{1}} \stackrel{\sim}{\rightarrow} \Phi_{2}\left(A_{2}\right)_{\mathfrak{p}_{2}}, \quad \Phi_{1}\left(A_{1}\right)_{\mathfrak{p}_{1}} \stackrel{\sim}{\rightarrow} \Phi_{2}\left(A_{2}\right)_{\mathfrak{p}_{2}}
$$

which we shall refer to, respectively, as the right-hand and left-hand isomorphisms induced by $\Psi$ (cf. Example 4.3 below).

Proof. First, we observe that by Proposition 1.10(vi) that every group-like object is Frobenius-trivial, hence, in particular, Div-Frobenius-trivial; moreover, (by the definition of a 'group-like object') every endomorphism of a group-like object is a Div-identity endomorphism, and every pre-step to or from a group-like object is an isomorphism (cf. Propositions 1.4(i), (iii) and 1.8(iii)). Thus, since $\Psi$ preserves non-group-like objects (cf. Theorem 3.4(ii)) and pull-back morphisms (cf. Theorem 3.4(iii)), we may assume for the remainder of the proof of Theorem 4.2, without loss of generality, that the objects under consideration are non-group-like. Now by Proposition 1.14(v) (cf. also Theorem 3.4(ii)), it follows immediately that $\Psi$ preserves non-group-like Div-Frobenius-trivial objects, as well as Div-identity prime-Frobenius endomorphisms of such objects. Since $\Psi$ preserves morphisms of Frobenius type and Frobenius degrees (cf. Theorem 3.4(iii)), we thus conclude that to complete the proof of assertion (i), it suffices to prove that $\Psi$ preserves primary steps and Div-identity endomorphisms. Moreover, to prove the remainder of assertion (i) (i.e., that $\Psi$ preserves primary steps and Div-identity endomorphisms) and assertions (ii) and (iii), clearly it suffices to do so after passing to the perfections of the $\mathcal{C}_{i}$ (cf. Theorem 3.4(iii)); thus, for the remainder of the proof of Theorem 4.2, we may assume, without loss of generality, that the $\mathcal{C}_{i}$ are of perfect type (cf. also Proposition 5.5(iii), below).

Now let $A_{1} \in \operatorname{Ob}\left(\mathcal{C}_{1}\right)$ be a non-group-like Div-Frobenius-trivial object and let $A_{2} \stackrel{\text { def }}{=}$ $\Psi\left(A_{1}\right)$. Then it follows formally from Proposition 4.1(i), (ii) (cf. also Theorem 3.4(ii), (iii)) that $\Psi$ maps primary steps to or from $A_{1}$ to primary steps to or from $A_{2}$ in such a way that primary steps $B_{1} \rightarrow A_{1}, A_{1} \rightarrow C_{1}$ with primary composite $B_{1} \rightarrow C_{1}$ are mapped to primary steps $B_{2} \rightarrow A_{2}, A_{2} \rightarrow C_{2}$ with primary composite $B_{2} \rightarrow C_{2}$. Next, let

$$
A_{1} \rightarrow F_{1}
$$

be a primary step. Then it follows immediately from Proposition 4.1(iii), together with what we have already shown concerning primary steps to or from $A_{1}$, that $\Psi$ maps primary steps to or from $F_{1}$ to primary steps to or from $F_{2} \stackrel{\text { def }}{=} \Psi\left(F_{1}\right)$ in such a way that primary steps $F_{1}^{\prime} \rightarrow F_{1}$, $F_{1} \rightarrow F_{1}^{\prime \prime}$ with primary composite $F_{1}^{\prime} \rightarrow F_{1}^{\prime \prime}$ are mapped to primary steps $F_{2}^{\prime} \rightarrow F_{2}, F_{2} \rightarrow F_{2}^{\prime \prime}$ with primary composite $F_{2}^{\prime} \rightarrow F_{2}^{\prime \prime}$. (Indeed, to see this, it suffices to consider the following two situations (depending on whether the primary steps $A_{1} \rightarrow F_{1}, F_{1}^{\prime} \rightarrow F_{1}$ are co-primary or not): (a) primary steps $B_{i} \rightarrow A_{i}, A_{i} \rightarrow C_{i}$ with primary composite such that the primary steps to or from $F_{i}$ under consideration are subordinate to the primary composite $B_{i} \rightarrow C_{i}$; 
(b) commutative diagrams

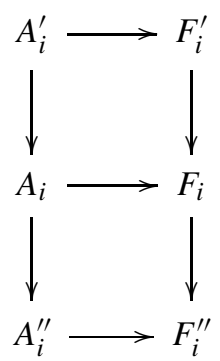

(where $i=1,2$ ) in which both the upper and lower squares are cartesian diagrams as in Proposition 4.1(iii), and all the arrows originating from $A_{i}$, as well as the vertical composite $A_{i}^{\prime} \rightarrow A_{i} \rightarrow A_{i}^{\prime \prime}$, are primary steps.)

Next, observe that for a suitable choice of non-group-like Div-Frobenius-trivial $A_{1}$ (e.g., a Frobenius-trivial $A_{1}-$ cf. Definition 1.3(i)(a), (b)), it follows that for any object $C_{1} \in$ $\mathrm{Ob}\left(\mathcal{C}_{1}\right)$ that is base-isomorphic to $A_{1}$, there exist pre-steps $B_{1} \rightarrow C_{1}, B_{1} \rightarrow A_{1}$. Moreover, observe that (by applying the equivalences of categories of Definition 1.3(iii)(d)) any primary step to or from $B_{1}$, as well as any primary composite of a primary step to $B_{1}$ with a primary step from $B_{1}$, may always be written in the form

$$
D_{1} \rightarrow E_{1},
$$

where the composite $D_{1} \rightarrow E_{1} \rightarrow F_{1}$ of the above arrow $D_{1} \rightarrow E_{1}$ with some arrow $E_{1} \rightarrow$ $F_{1}$ factors as a composite $D_{1} \rightarrow B_{1} \rightarrow A_{1} \rightarrow F_{1}$ in which $D_{1} \rightarrow B_{1}$ is a pre-step, $B_{1} \rightarrow A_{1}$ is the pre-step introduced above, and $A_{1} \rightarrow F_{1}$ is a primary step (so in the case of a primary step from $B_{1}, D_{1}=B_{1}$ and in the case of a primary step to $B_{1}, E_{1}=B_{1}$ ). Thus, by applying Proposition 4.1(iv) (to the arrows $D_{1} \rightarrow E_{1} \rightarrow F_{1}$ ), together with what we have already shown concerning primary steps to or from $F_{1}$, we conclude that $\Psi$ maps primary steps to or from $B_{1}$ to primary steps to or from $B_{2} \stackrel{\text { def }}{=} \Psi\left(B_{1}\right)$ in such a way that primary steps $B_{1}^{\prime} \rightarrow B_{1}, B_{1} \rightarrow B_{1}^{\prime \prime}$ with primary composite $B_{1}^{\prime} \rightarrow B_{1}^{\prime \prime}$ are mapped to primary steps $B_{2}^{\prime} \rightarrow B_{2}, B_{2} \rightarrow B_{2}^{\prime \prime}$ with primary composite $B_{2}^{\prime} \rightarrow B_{2}^{\prime \prime}$.

In a similar vein, we observe that (by applying the equivalences of categories of Definition 1.3(iii)(d)) a primary step to or from $C_{1}$, as well as any primary composite of a primary step to $C_{1}$ with a primary step from $C_{1}$, may always be written in the form

$$
E_{1} \rightarrow F_{1},
$$

where the composite $D_{1} \rightarrow E_{1} \rightarrow F_{1}$ of some arrow $D_{1} \rightarrow E_{1}$ with the above arrow $E_{1} \rightarrow$ $F_{1}$ factors as a composite $D_{1} \rightarrow B_{1} \rightarrow C_{1} \rightarrow F_{1}$ in which $D_{1} \rightarrow B_{1}$ is a primary pre-step, $B_{1} \rightarrow C_{1}$ is the pre-step introduced above, and $C_{1} \rightarrow F_{1}$ is a pre-step (so in the case of a primary step from $C_{1}, E_{1}=C_{1}$; in the case of a primary step to $C_{1}, F_{1}=C_{1}$ ). Thus, by applying Proposition 4.1(v) (to the arrows $D_{1} \rightarrow E_{1} \rightarrow F_{1}$ ), together with what we have already shown concerning primary steps to or from $D_{1}$ (i.e., where we regard ' $D_{1}$ ' as a 'sort of $B_{1}$ ', which is possible in light of the existence of the composite pre-step $D_{1} \rightarrow B_{1} \rightarrow A_{1}$ ), we conclude that $\Psi$ maps primary steps to or from $C_{1}$ to primary steps to or from $C_{2} \stackrel{\text { def }}{=} \Psi\left(C_{1}\right)$ in such a way that primary steps $C_{1}^{\prime} \rightarrow C_{1}, C_{1} \rightarrow C_{1}^{\prime \prime}$ with primary composite $C_{1}^{\prime} \rightarrow C_{1}^{\prime \prime}$ are mapped to primary steps $C_{2}^{\prime} \rightarrow C_{2}, C_{2} \rightarrow C_{2}^{\prime \prime}$ with primary composite $C_{2}^{\prime} \rightarrow C_{2}^{\prime \prime}$. 
Since $C_{1}$ was, in effect, allowed to be an arbitrary non-group-like object of $\mathcal{C}_{1}$, we thus conclude that $\Psi$ preserves primary steps. Moreover, by thinking, for $A_{i} \in \mathrm{Ob}\left(\mathcal{C}_{i}\right)$ (where $i=1,2)$ of an element of Prime $\left(\Phi_{i}\left(A_{i}\right)\right)$ as an equivalence class of primary steps to or from $A_{i}$ (where the correspondence between elements of Prime $\left(\Phi_{i}\left(A_{i}\right)\right)$ and equivalence classes of primary steps is defined by ' $\operatorname{Div}(-)$ ' - cf. the equivalences of categories of Definition 1.3(iii)(d)), we thus obtain that $\Psi$ induces a bijection

$$
\Psi^{\text {Prime }}\left(A_{1}\right): \operatorname{Prime}\left(\Phi_{1}\left(A_{1}\right)\right) \stackrel{\sim}{\rightarrow} \operatorname{Prime}\left(\Phi_{2}\left(A_{2}\right)\right)
$$

(where $A_{2} \stackrel{\text { def }}{=} \Psi\left(A_{1}\right)$ ) as well as corresponding equivalences of categories

$$
\left\{A_{1}\left(\mathcal{C}_{1}^{\text {coa-pre }}\right)\right\}_{\mathfrak{p}_{1}} \stackrel{\sim}{\rightarrow}\left\{A_{2}\left(\mathcal{C}_{2}^{\text {coa-pre }}\right)\right\}_{\mathfrak{p}_{2}}, \quad\left\{\left(\mathcal{C}_{1}^{\text {coa-pre }}\right)_{A_{1}}\right\}_{\mathfrak{p}_{1}} \stackrel{\sim}{\rightarrow}\left\{\left(\mathcal{C}_{2}^{\text {coa-pre }}\right)_{A_{2}}\right\}_{\mathfrak{p}_{2}}
$$

(where $\mathfrak{p}_{1} \in \operatorname{Prime}\left(\Phi_{1}\left(A_{1}\right)\right), \mathfrak{p}_{2} \in \operatorname{Prime}\left(\Phi_{2}\left(A_{2}\right)\right.$ ) correspond via $\Psi^{\text {Prime }}\left(A_{1}\right)$ ).

To check the functoriality of $\Psi^{\text {Prime }}(-)$ with respect to arbitrary base-isomorphisms, it suffices to check it with respect to morphisms of Frobenius type and pre-steps (cf. Proposition 1.7(ii)). In the case of a morphism of Frobenius type $B_{i} \rightarrow A_{i}$ (where $i=1,2$ ), the desired functoriality follows by considering commutative diagrams

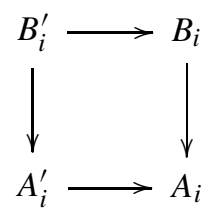

(cf. Proposition 1.10(i)), where the vertical morphisms are morphisms of Frobenius type and the horizontal morphisms are primary steps. In the case of a pre-step $B_{i} \rightarrow A_{i}$ (where $i=1,2$ ), the desired functoriality follows by considering a commutative diagram

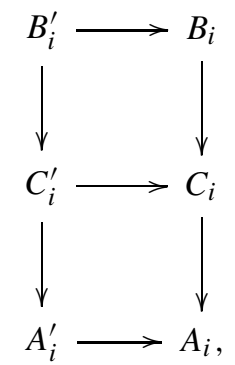

where all of the morphisms are pre-steps, all of the horizontal morphisms, as well as the vertical morphisms and composite morphisms of the upper square, are either isomorphisms or primary steps and either the vertical morphisms of the lower square are isomorphisms, or the lower square is a cartesian diagram as in Proposition 4.1(iii). This completes the proof of the functoriality of $\Psi^{\text {Prime }}(-)$, hence of assertion (ii).

Next, we observe that $\Psi$ preserves Div-identity endomorphisms. Indeed, since the $\Phi_{i}$ are non-dilating, it follows that if $A \in \operatorname{Ob}\left(\mathcal{C}_{i}\right)$ (where $i=1,2$ ), then $\alpha \in \operatorname{End}_{\mathcal{C}_{i}}(A)$ is a Dividentity endomorphism if and only if $\alpha$ admits a factorization $\alpha=\beta \circ \gamma$, where $\beta: B \rightarrow A$ is a pull-back morphism and $\gamma: A \rightarrow B$ is a base-isomorphism, such that for every primary 
step $A^{\prime} \rightarrow A$, there exists a commutative diagram

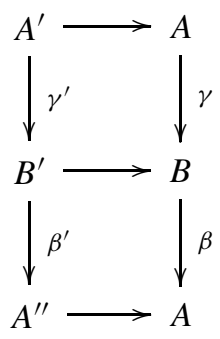

in which the horizontal morphisms are primary steps, the upper horizontal morphism is the given primary step, the equivalence classes of the primary steps $A^{\prime} \rightarrow A, B^{\prime} \rightarrow B$ correspond via the bijection Prime $\left(\Phi_{i}(\gamma)\right): \operatorname{Prime}\left(\Phi_{i}(B)\right) \stackrel{\sim}{\rightarrow} \operatorname{Prime}\left(\Phi_{i}(A)\right)$ (cf. the functoriality of $\left.\Psi^{\text {Prime }}(-)\right), \beta^{\prime}$ is a pull-back morphism (cf. Proposition $1.11(\mathrm{v})$ ) and the primary steps $A^{\prime} \rightarrow A, A^{\prime \prime} \rightarrow A$ determine the same element of Prime $\left(\Phi_{i}(A)\right)$. This completes the proof of assertion (i).

Finally, we consider assertion (iii). Thus, we assume that the $A_{i}$ are Div-Frobeniustrivial. By considering commutative diagrams of the form

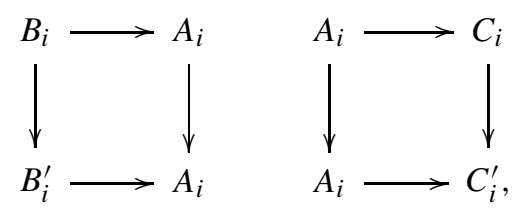

where the vertical morphisms are morphisms of Frobenius type (cf. Proposition 1.10(i)), the morphisms $A_{i} \rightarrow A_{i}$ are Div-identity endomorphisms and the horizontal morphisms are primary steps, it follows that the equivalences of categories in question arise from bijections of sets

$$
\Phi_{1}\left(A_{1}\right)_{\mathfrak{p}_{1}} \stackrel{\sim}{\rightarrow} \Phi_{2}\left(A_{2}\right)_{\mathfrak{p}_{2}}, \quad \Phi_{1}\left(A_{1}\right)_{\mathfrak{p}_{1}} \stackrel{\sim}{\rightarrow} \Phi_{2}\left(A_{2}\right)_{\mathfrak{p}_{2}}
$$

that are compatible both with ' $\leq$ ' and with multiplication by elements of $\mathbb{N}_{\geq 1}$. In light of the well-known structure of the monoids $\mathbb{Q}_{\geq 0}, \mathbb{R}_{\geq 0}$ (cf. Definition 2.4(i)(b)), this is enough to conclude that these bijections of sets are, in fact, isomorphisms of monoids, as desired. This completes the proof of assertion (iii).

Example 4.3. (Independence of right-hand and left-hand isomorphisms) As the following example shows, the right-hand and left-hand isomorphisms of Theorem 4.2(iii) do not necessarily coincide (cf. Remark 4.9.1 below): let $\mathcal{D}$ be a one-morphism category and let $\Phi$ be the monoid on $\mathcal{D}$ whose value on the unique object of $\mathcal{D}$ is the monoid $\mathbb{Q}_{\geq 0}$. Now we define a category $\mathcal{C}$ as follows. The objects of $\mathcal{C}$ are the elements of $\mathbb{Q}$. The morphisms $a \rightarrow b$ of $\mathcal{C}$ from an object $a \in \mathbb{Q}$ to an object $b \in \mathbb{Q}$ are the elements $d \in \mathbb{N}_{\geq 1}$ such that $d \cdot a \leq b$ and composition of morphisms is defined by multiplication of elements of $\mathbb{N}_{\geq 1}$. We shall refer to the element $d \in \mathbb{N}_{\geq 1}$ determined by a morphism of $\mathcal{C}$ as the Frobenius degree of the morphism. Thus, we obtain a natural functor

$$
\mathcal{C} \rightarrow \mathbb{F}_{\Phi}
$$


by assigning to a morphism $\phi: a \rightarrow b$ (where $a, b \in \mathbb{Q}$ ) the zero divisor $b-\operatorname{deg}_{\mathrm{Fr}}(\phi)$. $a \in \mathbb{Q}_{\geq 0}$ and Frobenius degree $\operatorname{deg}_{\mathrm{Fr}}(\phi) \in \mathbb{N}_{\geq 1}$. Since $\mathcal{C}$ is clearly connected and totally epimorphic, this functor determines a pre-Frobenioid structure on $\mathcal{C}$. Moreover, the object $0 \in \mathbb{Q}$ is Frobenius-trivial, $\phi: a \rightarrow b$ is a morphism of Frobenius type if and only if $b=$ $\operatorname{deg}_{\mathrm{Fr}}(\phi) \cdot a, \phi: a \rightarrow b$ is a pre-step if and only if $\operatorname{deg}_{\mathrm{Fr}}(\phi)=1$, all morphisms of $\mathcal{C}$ are baseisomorphisms, all pull-back morphisms of $\mathcal{C}$ are isomorphisms, all ' $\mathrm{O}^{\triangleright}(-)$ ' of $\mathcal{C}$ are trivial and no object of $\mathcal{C}$ is group-like. Thus, one verifies immediately that $\mathcal{C}$ is a Frobenioid of isotropic type. Since $\mathcal{D}$ is clearly of FSMFF-type, and $\Phi$ is non-dilating, it follows that $\mathcal{C}$ is also of standard type, over a slim base category $\mathcal{D}$. Now one verifies immediately that if $\lambda \in \mathbb{Q}_{>0}$, then the assignment, for $a \in \mathbb{Q}_{\geq 0}$,

$$
a \mapsto a, \quad-a \mapsto-\lambda \cdot a
$$

determines a self-equivalence of categories

$$
\Psi_{\lambda}: \mathcal{C} \stackrel{\sim}{\rightarrow} \mathcal{C}
$$

that preserves Frobenius degrees (cf. Theorem 3.4(iii)). On the other hand, it follows immediately from the construction of $\Psi_{\lambda}$ that the right-hand isomorphism of Theorem 4.2(iii) is the identity on $\mathbb{Q}_{\geq 0}$, while the left-hand isomorphism of Theorem 4.2(iii) is given by multiplication by $\lambda$ on $\mathbb{Q} \geq 0$.

In order to proceed further toward the goal of 'reconstructing $\Phi$ category-theoretically from $\mathcal{C}$ ', it is necessary to find natural conditions on the Frobenioid $\mathcal{C}$ that will allow us to rule out 'pathologies' of the sort discussed in Example 4.3. One approach to doing this is the introduction of the birationalization of a Frobenioid, as follows.

Proposition 4.4. (Birationalization of a Frobenioid I) For $A, B \in \mathrm{Ob}(\mathcal{C})$, write

$$
\operatorname{Hom}_{\mathcal{C}}^{\text {birat }}(A, B) \stackrel{\text { def }}{=} \underset{\left(A^{\prime} \rightarrow A\right) \in \mathcal{C}_{A}^{\text {coa-pre }}}{\lim _{\mathcal{C}}} \operatorname{Hom}_{\mathcal{C}}\left(A^{\prime}, B\right),
$$

where the inductive limit is parametrized by (say, some small skeletal subcategory of) $\mathcal{C}_{A}^{\text {coa-pre }}$, and the transition morphism induced by a co-angular pre-step $A^{\prime \prime} \rightarrow A^{\prime}$ (regarded as a morphism in $\mathcal{C}_{A}^{\text {coa-pre }}$ ) is the natural morphism $\operatorname{Hom}_{\mathcal{C}}\left(A^{\prime}, B\right) \rightarrow \operatorname{Hom}_{\mathcal{C}}\left(A^{\prime \prime}, B\right)$. Then we have the following.

(i) Composition of morphisms in $\mathcal{C}$ determines a natural composition map

$$
\operatorname{Hom}_{\mathcal{C}}^{\text {birat }}(A, B) \times \operatorname{Hom}_{\mathcal{C}}^{\text {birat }}(B, C) \rightarrow \operatorname{Hom}_{\mathcal{C}}^{\text {birat }}(A, C)
$$

(where $A, B, C \in \mathrm{Ob}(\mathcal{C})$ ), hence a category $\mathcal{C}^{\text {birat }}$, whose objects are the objects of $\mathcal{C}$ and whose morphisms are given by ' $\mathrm{Hom}_{\mathcal{C}}^{\text {birat }}$. Moreover, there exists a natural 1-commutative diagram of functors

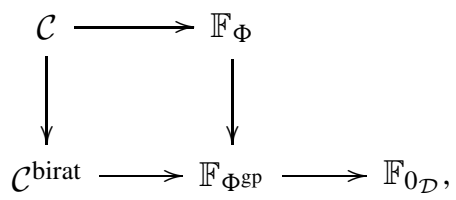


where the functors between elementary Frobenioids are those induced by the natural morphisms of monoids $\Phi \rightarrow \Phi^{\mathrm{gp}} \rightarrow 0_{\mathcal{D}}$ and $0_{\mathcal{D}}$ is the monoid on $\mathcal{D}$ all of whose values on objects of $\mathcal{D}$ are equal to the monoid with one element (so $\mathbb{F}_{0_{\mathcal{D}}}$ is the product category of $\mathcal{D}$ with the one-object category determined by the monoid $\mathbb{N}_{\geq 1}$ ).

(ii) The functor $\mathcal{C}^{\text {birat }} \rightarrow \mathbb{F}_{0_{\mathcal{D}}}$ of (i) determines a structure of a Frobenioid of group-like type on $\mathcal{C}^{\text {birat }}$. Moreover, the functor $\mathcal{C} \rightarrow \mathcal{C}^{\text {birat }}$ is faithful. In particular, for every $A \in \mathrm{Ob}(\mathcal{C})$ with image $A^{\text {birat }}$ in $\mathcal{C}^{\text {birat }}$, the functor $\mathcal{C} \rightarrow \mathcal{C}^{\text {birat }}$ determines an injection of groups $\mathcal{O}^{\triangleright}(A)^{\mathrm{gp}} \hookrightarrow \mathcal{O}^{\times}\left(A^{\text {birat }}\right)$. We shall refer to the functor ' $\mathcal{O}^{\times}(-)$' on $\mathcal{D}$ associated to the Frobenioid $\mathcal{C}^{\text {birat }}$ (cf. Proposition 2.2(ii), (iii)) as the rational function monoid of the Frobenioid $\mathcal{C}$.

(iii) There exists a unique subfunctor of groups $\Phi^{\text {birat }} \subseteq \Phi^{\mathrm{gp}}$ such that the functor $\mathcal{C}^{\text {birat }} \rightarrow$ $\mathbb{F}_{\Phi \mathrm{gp}}$ of (i) factors through the subcategory $\mathbb{F}_{\Phi^{\text {birat }}} \subseteq \mathbb{F}_{\Phi \mathrm{gp}}$ determined by $\Phi^{\text {birat }}$, and, moreover, the resulting functor

$$
\mathcal{C}^{\text {birat }} \rightarrow \mathbb{F}_{\Phi^{\text {birat }}}
$$

induces, for each $A^{\text {birat }} \in \mathrm{Ob}\left(\mathcal{C}^{\text {birat }}\right)$, a surjection $\mathcal{O}^{\times}\left(A^{\text {birat }}\right) \rightarrow \Phi^{\text {birat }}\left(A^{\text {birat }}\right)$, whose kernel is the image, via the injection $\mathcal{O}^{\triangleright}(A)^{\mathrm{gp}} \hookrightarrow \mathcal{O}^{\times}\left(A^{\text {birat }}\right)$ of (ii), of $\mathcal{O}^{\times}(A) \subseteq$ $\mathcal{O}^{\triangleright}(A)^{\mathrm{gp}}$.

(iv) A morphism of $\mathcal{C}$ maps to a(n) co-angular morphism (respectively, isomorphism; morphism of Frobenius type; pull-back morphism; morphism of a given Frobenius degree; isometry; pre-step; base-isomorphism) of $\mathcal{C}^{\text {birat }}$ if and only if it is a(n)coangular morphism (respectively, co-angular pre-step; co-angular base-isomorphism; co-angular linear morphism; morphism of a given Frobenius degree; arbitrary morphism; pre-step; base-isomorphism) of $\mathcal{C}$. A morphism of $\mathcal{C}^{\text {birat }}$ is a base-identity endomorphism if and only if it arises from a pair $\left(\alpha: A^{\prime} \rightarrow A ; \phi: A^{\prime} \rightarrow A\right)$, where $\alpha$ is a co-angular pre-step in the indexing category of the inductive limit defining $\operatorname{Hom}_{\mathcal{C}}^{\text {birat }}(A, A)$ and $\alpha$ and $\phi$ are base-equivalent. An object of $\mathcal{C}$ maps to an isotropic object of $\mathcal{C}^{\text {birat }}$ if and only if it is an isotropic object of $\mathcal{C}$.

Proof. First, we consider assertion (i). Given morphisms $\phi^{\prime}: A^{\prime} \rightarrow B, \psi^{\prime}: B^{\prime} \rightarrow C$ (in $\mathcal{C}$ ) and co-angular pre-steps $\alpha: A^{\prime} \rightarrow A, \beta: B^{\prime} \rightarrow B$ (in $\mathcal{C}$ ), it follows from Proposition 1.11(vii) that there exists a commutative diagram

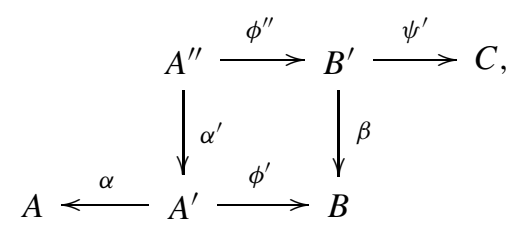

where $\alpha^{\prime}$ (hence also $\alpha \circ \alpha^{\prime}$ ) is a co-angular pre-step. Then we take the composite of the image of $\phi^{\prime}$ in $\operatorname{Hom}_{\mathcal{C}}^{\text {birat }}(A, B)$ with the image of $\psi^{\prime}$ in $\operatorname{Hom}_{\mathcal{C}}^{\text {birat }}(B, C)$ to be the image of $\psi^{\prime} \circ \phi^{\prime \prime}$ in $\operatorname{Hom}_{\mathcal{C}}^{\text {birat }}(A, C)$. To show that this assignment is independent of the choice of $\alpha^{\prime}$, 
$\phi^{\prime \prime}$, it suffices to consider commutative diagrams

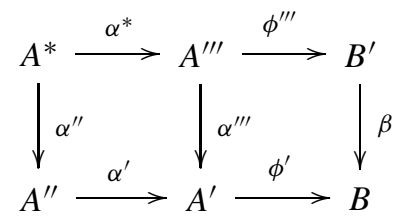

(where $\alpha^{\prime \prime}, \alpha^{\prime \prime \prime}, \alpha^{*}$ are co-angular pre-steps) and to observe that since $\beta$ is a monomorphism (cf. Definition 1.3(v)(a)), the fact that

$$
\beta \circ \phi^{\prime \prime \prime} \circ \alpha^{*}=\phi^{\prime} \circ \alpha^{\prime \prime} \circ \alpha^{*}=\phi^{\prime} \circ \alpha^{\prime} \circ \alpha^{\prime \prime}=\beta \circ \phi^{\prime \prime} \circ \alpha^{\prime \prime}
$$

implies that $\phi^{\prime \prime \prime} \circ \alpha^{*}=\phi^{\prime \prime} \circ \alpha^{\prime \prime}$, i.e., that $\psi^{\prime} \circ \phi^{\prime \prime}, \psi^{\prime} \circ \phi^{\prime \prime \prime}$ determine the same element of $\operatorname{Hom}_{\mathcal{C}}^{\text {birat }}(A, C)$. Also, one verifies immediately that composite of morphisms of $\operatorname{Hom}_{\mathcal{C}}^{\text {birat }}(-,-)$ is associative. This completes the definition of the category $\mathcal{C}^{\text {birat }}$. Then by assigning to the pair $\left(\alpha: A^{\prime} \rightarrow A, \phi^{\prime}: A^{\prime} \rightarrow B\right)$ the element

$$
\Phi(\alpha)^{-1}\left\{\operatorname{Div}\left(\phi^{\prime}\right)-\operatorname{deg}_{\mathrm{Fr}}\left(\phi^{\prime}\right) \cdot \operatorname{Div}(\alpha)\right\} \in \Phi(A)^{\mathrm{gp}}
$$

(cf. Remark 1.1.1) one verifies immediately that the functor $\mathcal{C} \rightarrow \mathbb{F}_{\Phi}$ induces a functor $\mathcal{C}^{\text {birat }} \rightarrow \mathbb{F}_{\Phi \text { gp }}$, as well as a 1-commutative diagram as in the statement of assertion (i). This completes the proof of assertion (i).

Next, we observe that it follows formally from the definition of $\mathcal{C}^{\text {birat }}$ that $\mathcal{C}^{\text {birat }}$ is connected; moreover, (cf. the discussion of the composition of arrows of $\mathcal{C}^{\text {birat }}$ in the proof of assertion (i)) the total epimorphicity of $\mathcal{C}^{\text {birat }}$ follows immediately from that of $\mathcal{C}$. Thus, the functor $\mathcal{C}^{\text {birat }} \rightarrow \mathbb{F}_{0_{\mathcal{D}}}$ determines a structure of a pre-Frobenioid on $\mathcal{C}^{\text {birat }}$. Now the portion of assertion (iv) concerning morphisms of a given Frobenius degree, isometries (cf. the monoid structure of the monoid $0_{\mathcal{D}}$ !), pre-steps, base-isomorphisms and baseidentity endomorphisms of $\mathcal{C}^{\text {birat }}$ follows immediately from the definitions. The portion of assertion (iv) concerning co-angular pre-steps of $\mathcal{C}$ follows immediately from the definition of ' $\operatorname{Hom}_{\mathcal{C}}^{\text {birat }}(-,-)$ '; Proposition 1.7(v) (for co-angular pre-steps).

To verify the portion of assertion (iv) concerning co-angular morphisms, we reason as follows. Given a morphism $A^{\text {birat }} \rightarrow B^{\text {birat }}$ in $\mathcal{C}^{\text {birat, }}$, any factorization $A^{\text {birat }} \rightarrow$ $C^{\text {birat }} \rightarrow D^{\text {birat }} \rightarrow B^{\text {birat }}$ in $\mathcal{C}^{\text {birat }}$, where either $A^{\text {birat }} \rightarrow C^{\text {birat }}$ or $D^{\text {birat }} \rightarrow B^{\text {birat }}$ is a baseisomorphism, $C^{\text {birat }} \rightarrow D^{\text {birat }}$ is a(n) (isometric) pre-step and $D^{\text {birat }} \rightarrow B^{\text {birat }}$ is linear, arises (cf. the proof of assertion (i)) from a factorization $A^{\prime} \rightarrow C^{\prime} \rightarrow D^{\prime} \rightarrow B$ in $\mathcal{C}$, where either $A^{\prime} \rightarrow C^{\prime}$ or $D^{\prime} \rightarrow B$ is a base-isomorphism, $C^{\prime} \rightarrow D^{\prime}$ is a pre-step and $D^{\prime} \rightarrow B$ is linear. Thus, if $A^{\prime} \rightarrow B$ is co-angular, then (by applying the factorization of Definition $1.3(\mathrm{v})(\mathrm{b})$ to $C^{\prime} \rightarrow D^{\prime}$, we conclude that) $C^{\prime} \rightarrow D^{\prime}$ is a co-angular pre-step, so $C^{\text {birat }} \rightarrow D^{\text {birat }}$ is an isomorphism; in particular, it follows that $A^{\text {birat }} \rightarrow B^{\text {birat }}$ is co-angular. On the other hand, if $A^{\text {birat }} \rightarrow B^{\text {birat }}$ is co-angular, then $C^{\text {birat }} \rightarrow D^{\text {birat }}$ is an isomorphism, which (by the portion of assertion (iv) concerning isomorphisms of $\mathcal{C}^{\text {birat }}$ ) implies that $C^{\prime} \rightarrow D^{\prime}$ is a co-angular pre-step, hence an isomorphism whenever it is an isometry (cf. Proposition 1.4(iii)); thus, $A^{\prime} \rightarrow B$, hence also any morphism $A \rightarrow B$ appearing in a factorization $A^{\prime} \rightarrow A \rightarrow B$ (where $A^{\prime} \rightarrow A$ is a co-angular pre-step) is co-angular.

The portion of assertion (iv) concerning morphisms of Frobenius type now follows formally from the portion of assertion (iv) concerning co-angular morphisms, isometries, 
and base-isomorphisms. Next, let us observe that it is immediate from the definition of a pull-back morphism (cf. Definition 1.2(ii)) that any pull-back morphism of $\mathcal{C}$ maps to a pullback morphism of $\mathcal{C}^{\text {birat }}$. Since, moreover, a morphism of $\mathcal{C}$ is a co-angular linear morphism if and only if it is a composite of a co-angular pre-step and a pull-back morphism (cf. Propositions 1.4(iv) and 1.7(iii)), it thus follows (cf. the portion of assertion (iv) concerning co-angular pre-steps of $\mathcal{C}$ ) that every co-angular linear morphism of $\mathcal{C}$ maps to a pull-back morphism of $\mathcal{C}^{\text {birat }}$. On the other hand, if $\phi: A \rightarrow B$ is a morphism of $\mathcal{C}$ that maps to a pull-back morphism $\phi^{\text {birat }}: A^{\text {birat }} \rightarrow B^{\text {birat }}$ of $\mathcal{C}^{\text {birat }}$, then it follows that $\phi$ is linear, hence that it factors as a composite $\gamma \circ \alpha$, where $\alpha: A \rightarrow C$ is a pre-step and $\gamma: C \rightarrow B$ is a pullback morphism (cf. Proposition 1.7(iii)). Thus, we obtain an equation $\phi^{\text {birat }}=\gamma^{\text {birat }} \circ \alpha^{\text {birat }}$ in $\mathcal{C}^{\text {birat }}$, where $\phi^{\text {birat }}, \gamma^{\text {birat }}$ are pull-back morphisms and $\alpha^{\text {birat }}$ is a base-isomorphism; but (by the isomorphism of functors appearing in the definition of a 'pull-back morphism' in Definition 1.2(ii)) this implies formally that $\alpha^{\text {birat }}$ is an isomorphism, hence (by the portion of assertion (iv) concerning co-angular pre-steps of $\mathcal{C}$ ) that $\alpha$ is a co-angular prestep, as desired. Finally, the portion of assertion (iv) concerning isotropic objects follows immediately from the portion of assertion (iv) concerning pre-steps and co-angular pre-steps and Propositions 1.4(i), (iii) and 1.9(iv). This completes the proof of assertion (iv).

In light of the 'dictionary' provided by assertion (iv) (cf. also Proposition 1.4(iv) and the equivalence of categories of Proposition 1.9(ii)), it is now a routine exercise to check that $\mathcal{C}^{\text {birat }}$ is, in fact, a Frobenioid of group-like type. Moreover, it is immediate from the definitions (and the total epimorphicity of $\mathcal{C}$ ) that the functor $\mathcal{C} \rightarrow \mathcal{C}^{\text {birat }}$ is faithful and determines an injection $\mathcal{O}^{\triangleright}(A)^{\mathrm{gp}} \hookrightarrow \mathcal{O}^{\times}\left(A^{\text {birat }}\right)$, for $A \in \mathrm{Ob}(\mathcal{C})$. This completes the proof of assertion (ii).

Now assertion (iii) follows immediately from the existence of the functor $\mathcal{C}^{\text {birat }} \rightarrow \mathbb{F}_{\Phi \text { gp }}$ of assertion (i) (cf. also Proposition 1.5(ii)); here, we note that the computation of the kernel of the surjection of assertion (iii) follows from Definition 1.3(vi).

\section{Definition 4.5 .}

(i) We shall say that an object of $\mathcal{C}$ is birationally Frobenius-normalized if its image in $\mathcal{C}^{\text {birat }}$ is Frobenius-normalized. (Thus, any birationally Frobenius-normalized object of $\mathcal{C}$ is Frobenius-normalized - cf. Proposition 4.4(ii), (iv).) If every object of $\mathcal{C}$ is birationally Frobenius-normalized, then we shall say that $\mathcal{C}$ is of birationally Frobenius-normalized type. If $\mathcal{C}$ is of pre-model and birationally Frobenius-normalized type, then we shall say that $\mathcal{C}$ is of model type.

(ii) Suppose that $\Phi$ is perf-factorial and $A \in \mathrm{Ob}(\mathcal{C})$. Then we shall say that $A$ is strictly rational if, for every prime $\mathfrak{p} \in \operatorname{Prime}(\Phi(A))$, there exists an element $a-b \in \Phi^{\text {birat }}(A)$, where $a, b \in \Phi(A)$ such that $\mathfrak{p} \in \operatorname{Supp}(a), \mathfrak{p} \notin \operatorname{Supp}(b)$ (cf. Definition 2.4(i)(d)). We shall say that $A$ is rational if there exists a pull-back morphism $B \rightarrow A$ in $\mathcal{C}$, where $B$ is strictly rational. If ( $\Phi$ is perf-factorial and) every object of $\mathcal{C}$ is rational (respectively, strictly rational), then we shall say that $\mathcal{C}$ is of rational (respectively, strictly rational) type.

(iii) We shall say that $\mathcal{C}$ is of rationally standard type if the following conditions are satisfied: (a) $\mathcal{C}$ is of birationally Frobenius-normalized, rational and standard type; (b) $\left(\mathcal{C}^{\text {un-tr }}\right)^{\text {birat }}$ admits a Frobenius-compact object.

(iv) We shall say that $\mathcal{D}$ is Div-slim (relative to $\Phi$ ) if, for every $A \in \operatorname{Ob}(\mathcal{D})$, the homomorphism

$$
\operatorname{Aut}\left(\mathcal{D}_{A} \rightarrow \mathcal{D}\right) \rightarrow \operatorname{Aut}\left(\mathcal{D}_{A} \rightarrow \mathfrak{M o n}\right)
$$


(induced by composition with the functor $\Phi: \mathcal{D} \rightarrow \mathfrak{M o n}$ ) is injective. (Thus, if $\mathcal{D}$ is slim, then it is Div-slim.)

Remark 4.5.1. We observe in passing that it is immediate from the definitions that if $\mathcal{C}$ is of rationally standard type (respectively, of standard type), then so is $\mathcal{C}^{\text {istr }}$.

Example 4.6. (Frobenius-normalized versus birationally Frobenius-normalized) As the following example shows, it is not necessarily the case that a Frobenioid of Frobeniusnormalized type is of birationally Frobenius-normalized type. Let $G$ be an abelian group, written additively. For each $p \in \mathfrak{P r i m e s}$, let $\xi_{p} \in G$. Then if we write $M \stackrel{\text { def }}{=} G \times \mathbb{Z} \times \mathbb{Z}$, then the assignment

$$
M \ni(g, a, b) \mapsto\left(p \cdot g+a \cdot \xi_{p}, p \cdot a, p \cdot b\right) \in M
$$

determines an endomorphism $\alpha_{p} \in \operatorname{End}(M)$ of the module $M$ such that $\alpha_{p}$ commutes with all $\alpha_{p^{\prime}}$, for $p^{\prime} \in \mathfrak{P r i m e s}$. Thus, we obtain a homomorphism $\mathbb{N}_{\geq 1} \rightarrow \operatorname{End}(M)$, i.e., an action of $\mathbb{N}_{\geq 1}$ on $M$; write $\alpha_{n}$ for the image in $\operatorname{End}(M)$ of $n \in \mathbb{N}_{\geq 1}$. Write $N$ for the monoid whose underlying set is equal to the direct product

$$
M \times \mathbb{N}_{\geq 1}
$$

and whose monoid structure is given as follows. If $\lambda, \mu \in M$ and $l, m \in \mathbb{N}_{\geq 1}$, then $(\lambda, l)$. $(\mu, m)=\left(\lambda+\alpha_{l}(\mu), l \cdot m\right)$. Now let $\mathcal{D}$ be a one-morphism category and let $\Phi$ be the monoid on $\mathcal{D}$ whose unique value is given by $\mathbb{Z}_{\geq 0} \times \mathbb{Z}_{\geq 0}$. Let $\mathcal{C}$ be the category whose objects $A_{n}$ are indexed by elements $n \in \mathbb{Z}$ and whose morphisms $A_{n_{1}} \rightarrow A_{n_{2}}$ (where $n_{1}, n_{2} \in \mathbb{Z}$ ) consist of elements $(g, a, b, d) \in N$ such that $a \geq 0, b \geq 0, n_{2}-d \cdot n_{1}=a+b$; composition of morphisms is determined by the product structure of $N$. The assignment $(g, a, b, d) \mapsto$ $(a, b, d)$ then determines a functor

$$
\mathcal{C} \rightarrow \mathbb{F}_{\Phi}
$$

(which lies over $\mathcal{D}$ ). Moreover, one checks immediately that, relative to this last functor, $\mathcal{C}$ is a Frobenioid of isotropic and standard type which is not of group-like type. Also, we observe that the object $A_{0} \in \mathrm{Ob}(\mathcal{C})$ is Frobenius-trivial, and that for every $A \in \operatorname{Ob}(\mathcal{C})$, $\mathcal{O}^{\times}(A)=\mathcal{O}^{\triangleright}(A)=G$. On the other hand, one computes easily that for $A^{\text {birat }} \in \mathrm{Ob}\left(\mathcal{C}^{\text {birat }}\right)$, $\mathcal{O}^{\times}\left(A^{\text {birat }}\right)=M_{0}$, where we write $M_{0} \subseteq M$ for the subgroup of $(g, a, b) \in M$ such that $a+b=0$. Moreover, the morphisms $(0,0,0, d) \in N$ determine a homomorphism $\mathbb{N}_{\geq 1} \rightarrow$ $\operatorname{End}_{\mathcal{C}}\left(A_{0}\right) \rightarrow \operatorname{End}_{\mathcal{C}^{\text {birat }}}\left(A_{0}^{\text {birat }}\right)$ (where we use the superscript 'birat' to denote the image of objects of $\mathcal{C}$ in $\left.\mathcal{C}^{\text {birat }}\right)$, hence an action of $\mathbb{N}_{\geq 1}$ on $\mathcal{O}^{\times}\left(A^{\text {birat }}\right)=M_{0}$, which is easily verified to coincide with the restriction to $M_{0}$ of the original action of $\mathbb{N}_{\geq 1}$ on $M$. Now observe that $\mathcal{C}$ is of (strictly) rational type (cf. Definition 4.5(ii)) and, moreover, every object of $\left(\mathcal{C}^{\text {un-tr }}\right)^{\text {birat }}$ is Frobenius-compact. On the other hand, if the $\xi_{p} \neq 0$ (so $\alpha_{p}$ does not act on $M_{0}$ by multiplication by $p$ ), then $\mathcal{C}$ fails to be of birationally Frobenius-normalized type. (In a similar vein, we note that although $\mathcal{C}^{\text {birat }}$ is 'very similar' to an elementary Frobenioid, the presence of the ' $\xi_{p}$ ' means that it is not, in general, an elementary Frobenioid.)

Example 4.7. (Frobenius-slim versus Div-slim)

(i) Suppose that the functor $\Phi: \mathcal{D} \rightarrow \mathfrak{M o n}$ maps every automorphism of $\mathcal{D}$ to an identity automorphism of $\mathfrak{M o n}$. Then it follows formally that $\mathcal{D}$ is Div-slim if and only if $\mathcal{D}$ is slim. 
In particular, if, for instance, $\mathcal{D}$ is a one-object category, $A \in \mathrm{Ob}(\mathcal{D})$ and $\operatorname{End}_{\mathcal{D}}(A)$ is a nontrivial residually finite group $G$, then

$$
\operatorname{Aut}\left(\mathcal{D}_{A} \rightarrow \mathcal{D}\right)=\operatorname{Ker}\left(\operatorname{Aut}\left(\mathcal{D}_{A} \rightarrow \mathcal{D}\right) \rightarrow \operatorname{Aut}\left(\mathcal{D}_{A} \rightarrow \mathfrak{M o n}\right)\right)=G
$$

so (cf. Remark 3.1.2) D is Frobenius-slim, but not Div-slim.

(ii) Let $V \stackrel{\text { def }}{=} \mathbb{Q}, N \stackrel{\text { def }}{=}\left(\mathbb{N}_{\geq 1}\right)^{\text {gp }}$ and $G \stackrel{\text { def }}{=} V \rtimes N$, where $N(\subseteq \mathbb{Q})$ acts on $V$ multiplicatively. Let $\mathcal{D}$ be a one-object category, $A \in \mathrm{Ob}(\mathcal{D})$; suppose that $\operatorname{End}_{\mathcal{D}}(A)=G$ (so $\operatorname{Aut}\left(\mathcal{D}_{A} \rightarrow\right.$ $\mathcal{D})=G$ ). Then clearly there exists an injection $\mathbb{F} \hookrightarrow G$, so $\mathcal{D}$ fails to be Frobenius-slim. On the other hand, if $\Phi: \mathcal{D} \rightarrow \mathfrak{M o n}$ is the functor determined by the monoid

$$
\bigoplus_{g \in G} \mathbb{Z}_{\geq 0}
$$

(i.e., the copies of $\mathbb{Z}_{\geq 0}$ are indexed by the elements of $G$ ) equipped with the $G$-action obtained by letting $G$ act by left multiplication on the indices of the copies of $\mathbb{Z}_{\geq 0}$, then the natural map

$$
\operatorname{Aut}\left(\mathcal{D}_{A} \rightarrow \mathcal{D}\right)=G \rightarrow \operatorname{Aut}\left(\mathcal{D}_{A} \rightarrow \mathfrak{M o n}\right)
$$

is clearly injective, so $\mathcal{D}$ is Div-slim (relative to $\Phi$ ).

Proposition 4.8. (Birationalization of a Frobenioid II)

(i) If $\mathcal{C}$ is of isotropic type, then so is $\mathcal{C}^{\text {birat }}$.

(ii) If $\mathcal{C}$ is of perfect and isotropic type, then so is $\mathcal{C}^{\text {birat }}$.

(iii) If $\mathcal{C}$ is of rationally standard type, then $\left(\mathcal{C}^{\text {istr }}\right)^{\text {birat }}$ is of standard type.

(iv) If $\mathcal{C}$ is of isotropic and pre-model type, then so is $\mathcal{C}^{\text {birat }}$.

Proof. Assertion (i) follows formally from Proposition 4.4(iv). To prove assertion (ii), observe that the naive Frobenius functor (cf. Proposition 2.1) determines a natural 1commutative diagram (cf. Proposition 4.4(ii), (iv))

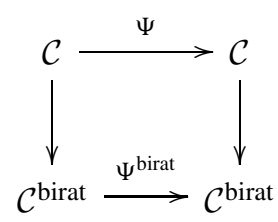

in which the vertical arrows are the natural functor $\mathcal{C} \rightarrow \mathcal{C}^{\text {birat }}$ of Proposition 4.4(i), the horizontal arrows are the 'naive Frobenius functor' of Proposition 2.1 and the upper horizontal arrow is an equivalence of categories (by our assumption that $\mathcal{C}$ is of perfect type and Proposition 2.1(iii)). Since, moreover, $\Psi$ and any quasi-inverse to $\Psi$ preserve (necessarily co-angular, since $\mathcal{C}$ is of isotropic type) pre-steps, it thus follows immediately (cf. the definition of ' $\mathrm{C}^{\text {birat'}}$ ') that $\Psi^{\text {birat }}$ is also an equivalence of categories. But this implies (cf. Proposition 2.1(iii)) that $\mathcal{C}^{\text {birat }}$ is of perfect type, as desired. In light of assertion (i), this completes the proof of assertion (ii). Finally, assertion (iii) follows formally from the definitions (cf. also assertion (i)) and assertion (iv) follows formally Proposition 4.4(iv) (cf. also assertion (i)). 
We are now ready to 'reconstruct $\Phi$ category-theoretically from $\mathcal{C}$ '.

THEOREM 4.9. (Category-theoreticity of divisor monoids) For $i=1$, 2, let $\Phi_{i}$ be a divisorial monoid on a connected, totally epimorphic category $\mathcal{D}_{i}$ let $\mathcal{C}_{i} \rightarrow \mathbb{F}_{\Phi_{i}}$ be a Frobenioid of rationally standard type and let

$$
\Psi: \mathcal{C}_{1} \stackrel{\sim}{\rightarrow} \mathcal{C}_{2}
$$

be an equivalence of categories. Then there exists an isomorphism of functors

$$
\Psi^{\Phi}: \Phi_{1} \stackrel{\sim}{\rightarrow} \Phi_{2}
$$

(where we regard, for $i=1,2$, the functor $\Phi_{i}: \mathcal{D}_{i} \rightarrow \mathfrak{M o n}$ as a functor on $\mathcal{C}_{i}$, by restriction via the natural projection functor $\mathcal{C}_{i} \rightarrow \mathcal{D}_{i}$ ) lying over $\Psi$, which is compatible (when the $\mathcal{C}_{i}$ are of isotropic, but not of group-like type) with the isomorphism $\Psi^{\text {Prime }}$ of Theorem 4.2(ii).

Proof. First, we observe (cf. Theorem 3.4(i), (ii)) that we may assume without loss of generality that $\mathcal{C}_{1}, \mathcal{C}_{2}$ are of isotropic type (cf. Remark 4.5.1) but not of group-like type (since Theorem 4.9 is vacuous if $\mathcal{C}_{1}, \mathcal{C}_{2}$ are of group-like type).

Next, I claim that to complete the proof of Theorem 4.9, it suffices to show that the right-hand and left-hand isomorphisms of Theorem 4.2(iii) coincide (cf. Remark 4.9.1 below) for all universally Div-Frobenius-trivial objects (e.g., Frobenius-trivial objects - cf. Remark 1.11.1). Indeed, if the right-hand and left-hand isomorphisms of Theorem 4.2(iii) coincide for all universally Div-Frobenius-trivial objects, then it follows immediately from the construction of the isomorphism of functors $\Psi^{\text {Prime }}$ in the proof of Theorem 4.2(ii) that $\Psi^{\text {Prime }}$ extends, for $A_{i} \in \operatorname{Ob}\left(\mathcal{C}_{i}^{\text {bs-iso }}\right), \mathfrak{p}_{i} \in \operatorname{Prime}\left(\Phi_{i}\left(A_{i}\right)\right)$ (where $i=1,2$ ) such that $A_{2}=\Psi\left(A_{1}\right), \mathfrak{p}_{2}=\Psi^{\text {Prime }}\left(\mathfrak{p}_{1}\right)$, to an isomorphism of monoids

$$
\Phi_{1}\left(A_{1}\right)_{\mathfrak{p}_{1}}^{\mathrm{pf}} \stackrel{\sim}{\rightarrow} \Phi_{2}\left(A_{2}\right)_{\mathfrak{p}_{2}}^{\mathrm{pf}}
$$

which is functorial in $A_{1}$ (regarded as an object of $\mathcal{C}_{1}^{\text {bs-iso }}$ ). Thus, by allowing the $\mathfrak{p}_{i}$ to vary, we obtain, for $A_{i} \in \mathrm{Ob}\left(\mathcal{C}_{i}^{\text {bs-iso }}\right.$ ) (where $\left.i=1,2\right)$ such that $A_{2}=\Psi\left(A_{1}\right)$, an isomorphism of monoids

$$
\Phi_{1}\left(A_{1}\right)_{\text {factor }}^{\mathrm{pf}} \stackrel{\sim}{\rightarrow} \Phi_{2}\left(A_{2}\right) \text { factor }
$$

(cf. Definition 2.4(i)(c)) which is functorial in $A_{1}$ (regarded as an object of $\mathcal{C}_{1}^{\text {bs-iso }}$ ). Moreover, by applying, say, the first equivalence of categories of Definition 1.3(iii)(d) to obtain presteps $\phi: A \rightarrow B$ of $\mathcal{C}_{i}$ with arbitrary prescribed zero divisor and considering primary steps $\psi: A \rightarrow C$ such that $\phi=\zeta \circ \psi$ for some pre-step $\zeta$, one concludes immediately that this subset maps the subset $\Phi_{1}\left(A_{1}\right) \subseteq \Phi_{1}\left(A_{1}\right)_{\text {factor }}^{\mathrm{pf}}$ (cf. Definition 2.4(i)(c)) onto the subset $\Phi_{2}\left(A_{2}\right) \subseteq \Phi_{2}\left(A_{2}\right)_{\text {factor }}^{\mathrm{pf}}$, hence determines an isomorphism of monoids

$$
\Phi_{1}\left(A_{1}\right) \stackrel{\sim}{\rightarrow} \Phi_{2}\left(A_{2}\right)
$$

which is functorial in $A_{1}$ (regarded as an object of $\mathcal{C}_{1}^{\text {bs-iso }}$ ). Finally, the functoriality of this isomorphism of monoids with respect to pull-back morphisms follows immediately by 'pulling back pre-steps', as in Proposition 1.11(v). This completes the proof of the claim.

To prove that the right-hand and left-hand isomorphisms of Theorem 4.2(iii) coincide for all universally Div-Frobenius-trivial objects, we reason as follows. First of all, by passing to perfections (cf. Theorem 3.4(iii)), we may assume without loss of generality 
that $\mathcal{C}_{1}, \mathcal{C}_{2}$ are of perfect type (cf. also Proposition 5.5(iii), below). Let $A$ be a universally Div-Frobenius-trivial object of $\mathcal{C}_{i}$ (where $i=1,2$ ). Since the right-hand and left-hand isomorphisms of Theorem 4.2(iii) are clearly compatible with pull-back morphisms (cf. Proposition 1.11(v) and the proof of Theorem 4.2(iii)) and $\Psi$ preserves pull-back morphisms (cf. Theorem 3.4(iii)), it follows that we may assume without loss of generality that $A$ is strictly rational. Let us refer to pairs of primary steps $\beta: A \rightarrow B, \gamma: C \rightarrow A$ such that $\operatorname{Div}(\beta)=\left(\Phi_{i}(\gamma)\right)^{-1}(\operatorname{Div}(\gamma))$ as twin-primary steps. Then, it suffices to show, for each $\mathfrak{p} \in \operatorname{Prime}\left(\Phi_{1}(A)\right)$, the existence of twin-primary steps with zero divisor in $\mathfrak{p}$ that are mapped by $\Psi$ to twin-primary steps of $\mathcal{C}_{2}$.

On the other hand, since $A$ is strictly rational, it follows (cf. Definition 4.5(ii)) that there exist, for each $\mathfrak{p} \in \operatorname{Prime}\left(\Phi_{i}(A)\right)$, cartesian commutative diagrams of pre-steps as in Proposition 4.1(iii),
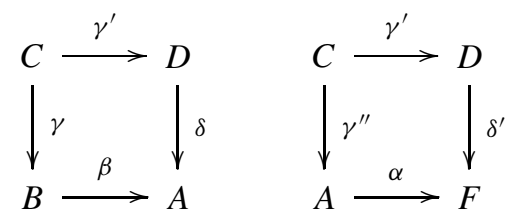

in which $\alpha, \beta$ are twin-primary with zero divisor in $\mathfrak{p}$ and the pre-steps $\zeta \stackrel{\text { def }}{=} \beta \circ \gamma: C \rightarrow$ $A, \gamma^{\prime \prime}: C \rightarrow A$ are Div-equivalent (e.g., base-equivalent). (Indeed, Definition 4.5(ii) (cf. also the equivalences of categories of Definition 1.3(iii)(d)) guarantees the existence of base-equivalent pre-steps $\zeta, \gamma^{\prime \prime}$ - which may, moreover, be taken to be co-primary (cf. Proposition 4.1(iii) and Definition 2.4(i)(c), (d)) by our assumption that $\mathcal{C}_{i}$ is of perfect typesuch that $\zeta$ admits a factorization $\beta \circ \gamma$, where $\beta$ is primary with zero divisor that maps via $\Phi(\beta)^{-1}$ to an element of $\mathfrak{p}$ and (again by our assumption that $\mathcal{C}_{i}$ is of perfect type) $\mathfrak{p}$ is not contained in the support of $(\Phi(\zeta))^{-1}(\operatorname{Div}(\gamma))$.) Conversely, given any pair of cartesian diagrams of pre-steps as in Proposition 4.1(iii),
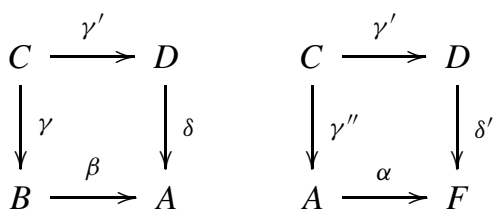

in which $\alpha, \beta$ are primary with zero divisor in $\mathfrak{p}$ and the pre-steps $\zeta \stackrel{\text { def }}{=} \beta \circ \gamma: C \rightarrow A$, $\gamma^{\prime \prime}: C \rightarrow A$ are Div-equivalent (e.g., base-equivalent), it follows immediately that $\alpha, \beta$ are twin-primary. On the other hand, since $\Psi$ preserves pre-steps (cf. Theorem 3.4(ii)), primary steps (cf. Theorem 4.2(i)), Div-equivalent pairs of base-isomorphisms (cf. Theorem 4.2(ii) and the fact that $\Phi_{i}$ is non-dilating) and cartesian diagrams as in Proposition 4.1(iii) (cf. Proposition 4.1(iii) or, alternatively, Theorem 4.2(ii)), we thus conclude that for each $\mathfrak{p} \in \operatorname{Prime}\left(\Phi_{1}(A)\right)$, there exist twin-primary steps with zero divisor in $\mathfrak{p}$ that are mapped by $\Psi$ to twin-primary steps of $\mathcal{C}_{2}$. This completes the proof of Theorem 4.9.

Remark 4.9.1. One verifies immediately that the Frobenioid of Example 4.3 is not of rational type. 
COROLlaRY 4.10. (Category-theoreticity of the birationalization) For $i=1$, 2, let $\Phi_{i}$ be a divisorial monoid on a connected, totally epimorphic category $\mathcal{D}_{i}$ of FSMFF-type let $\mathcal{C}_{i} \rightarrow \mathbb{F}_{\Phi_{i}}$ be a Frobenioid of quasi-isotropic type and let

$$
\Psi: \mathcal{C}_{1} \stackrel{\sim}{\rightarrow} \mathcal{C}_{2}
$$

be an equivalence of categories. Then there exists a 1 -unique functor $\Psi^{\text {birat }}: \mathcal{C}_{1}^{\text {birat }} \rightarrow \mathcal{C}_{2}^{\text {birat }}$ that fits into a 1-commutative diagram

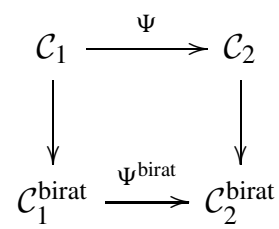

(where the vertical arrows are the natural functors of Proposition 4.4(i) and the horizontal arrows are equivalences of categories). Finally, if $\mathcal{D}_{1}, \mathcal{D}_{2}$ are slim and $\mathcal{C}_{1}, \mathcal{C}_{2}$ are of birationally Frobenius-normalized type, then each of the composite functors of this diagram is rigid.

Proof. The existence and 1-uniqueness of a 1-commutative diagram as in the statement of Corollary 4.10 follows immediately from the definition of ' $\mathcal{C}_{i}^{\text {birat }}$ ' (cf. Proposition 4.4(i)) and the fact that $\Psi$ preserves co-angular pre-steps (cf. Theorem 3.4(ii)). The rigidity assertion then follows immediately from Proposition 1.13(i) by considering base-identity endomorphisms of Frobenius type of Frobenius-trivial objects of $\mathcal{C}_{i}$, under the hypothesis that the $\mathcal{C}_{i}$ are birationally Frobenius-normalized (cf., e.g., the proof of the rigidity assertion of Theorem 3.4(i)).

COROLlary 4.11. (Category-theoreticity of the functor to an elementary Frobenioid I) For $i=1,2$, let $\Phi_{i}$ be a perf-factorial divisorial monoid on a connected, totally epimorphic category $\mathcal{D}_{i}$ which is Div-slim (with respect to $\Phi_{i}$ ), let $\mathcal{C}_{i} \rightarrow \mathbb{F}_{\Phi_{i}}$ be a Frobenioid of standard type and let

$$
\Psi: \mathcal{C}_{1} \stackrel{\sim}{\rightarrow} \mathcal{C}_{2}
$$

be an equivalence of categories. If $\mathcal{C}_{1}, \mathcal{C}_{2}$ are of group-like type, then we also assume that both $\Psi$ and some quasi-inverse to $\Psi$ preserve base-isomorphisms. Then we have the following.

(i) There exists a 1-unique functor $\Psi^{\mathrm{un}-\mathrm{tr}}: \mathcal{C}_{1}^{\mathrm{un}-\mathrm{tr}} \rightarrow \mathcal{C}_{2}^{\mathrm{un}-\mathrm{tr}}$ that fits into a 1-commutative diagram

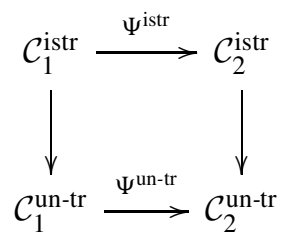

(where the vertical arrows are the natural projection functors, the horizontal arrows are equivalences of categories and $\Psi^{\mathrm{istr}}$ is the restriction of $\Psi$ to $\mathcal{C}_{1}^{\mathrm{istr}}-c f$. Theorem 3.4(i)). Moreover, each of the composite functors of this diagram is rigid. 
(ii) There exists a 1-unique functor $\Psi^{\text {Base }}: \mathcal{D}_{1} \rightarrow \mathcal{D}_{2}$ that fits into a 1-commutative diagram

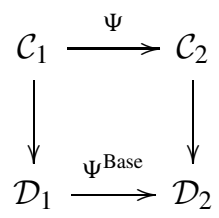

(where the vertical arrows are the natural projection functors and the horizontal arrows are equivalences of categories). Moreover, if $\mathcal{D}_{1}, \mathcal{D}_{2}$ are slim, then each of the composite functors of this diagram is rigid.

(iii) Suppose further that $\mathcal{C}_{1}, \mathcal{C}_{2}$ are of rationally standard type. Then there exists an isomorphism of functors

$$
\Psi^{\Phi}: \Phi_{1} \stackrel{\sim}{\rightarrow} \Phi_{2}
$$

(where we regard, for $i=1,2$, the functor $\Phi_{i}: \mathcal{D}_{i} \rightarrow \mathfrak{M o n}$ as a functor on $\mathcal{D}_{i}$ ) lying over the equivalence of categories $\Psi^{\text {Base }}$ of $(i)$, which is compatible (when the $\mathcal{C}_{i}$ are of isotropic, but not of group-like type) with the isomorphism $\Psi^{\text {Prime }}$ of Theorem 4.2(ii). In particular, $\Psi^{\text {Base }}, \Psi^{\Phi}$ induce an equivalence of categories $\Psi^{\mathbb{F}}: \mathbb{F}_{\Phi_{1}} \stackrel{\sim}{\rightarrow} \mathbb{F}_{\Phi_{2}}$.

(iv) Suppose further that $\mathcal{C}_{1}, \mathcal{C}_{2}$ are of rationally standard type. Then there exists a 1commutative diagram

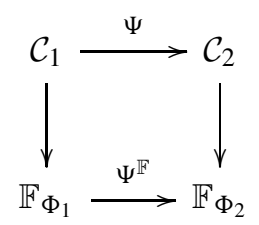

(where the vertical arrows are the functors that define the Frobenioid structures on $\mathcal{C}_{1}$, $\mathcal{C}_{2}$ and the horizontal arrows are equivalences of categories). Moreover, each of the composite functors of this diagram is rigid.

Proof. First, we observe (cf. Theorem 3.4(i)) that we may assume without loss of generality that $\mathcal{C}_{1}, \mathcal{C}_{2}$ are of isotropic type (cf. Remark 4.5.1). Also, if $\mathcal{C}_{1}, \mathcal{C}_{2}$ are of group-like type (cf. Theorem 3.4(ii)), then 'Div-slimness' amounts to 'slimness', so assertions (i) and (ii) follow from Theorem 3.4(iv) and (v); assertion (iii) is vacuous; assertion (iv) follows from the fact that $\Psi$ preserves Frobenius degrees (cf. Theorem 3.4(iii), (iv)). Thus, we may assume without loss of generality that $\mathcal{C}_{1}, \mathcal{C}_{2}$ are not of group-like type.

Now we consider assertion (i). To show the existence and 1-uniqueness of a 1commutative diagram as in the statement of assertion (i), it suffices to show that $\Psi$ preserves ' $\mathcal{O}^{\times}(-)$' (cf. the proof of Theorem 3.4(iv)). But observe that, for $A \in \mathrm{Ob}\left(\mathcal{C}_{i}\right)$, an element $f \in \mathcal{O}^{\times}(A)$ determines an automorphism (cf. the proof of Proposition 3.3(i))

$$
\phi_{f} \in \operatorname{Aut}\left(\left(\mathcal{C}_{i}^{\mathrm{pl}-\mathrm{bk}}\right)_{A} \rightarrow \mathcal{C}_{i}\right)
$$

that maps to the identity in $\operatorname{Aut}\left(\left(\mathcal{D}_{i}\right)_{A_{\mathcal{D}}} \rightarrow \mathcal{D}_{i}\right)$ (where $A_{\mathcal{D}} \stackrel{\text { def }}{=} \operatorname{Base}(A)-$ cf. the equivalence of categories $\left(\mathcal{C}_{i}^{\mathrm{pl}-\mathrm{bk}}\right)_{A} \stackrel{\sim}{\rightarrow}\left(\mathcal{D}_{i}\right)_{A_{\mathcal{D}}}$ of Definition 1.3(i)(c)), hence also to the identity in $\operatorname{Aut}\left(\left(\mathcal{D}_{i}\right)_{A_{\mathcal{D}}} \rightarrow \mathfrak{M o n}\right)$ (i.e., via composition with $\left.\Phi_{i}\right)$. Since $\mathcal{D}_{i}$ is Div-slim, it thus follows 
that the elements of $\mathcal{O}^{\times}(A) \subseteq \operatorname{Aut}_{\mathcal{C}_{i}}(A)$ may be characterized as the automorphisms of $A$ that arise from automorphisms

$$
\phi \in \operatorname{Aut}\left(\left(\mathcal{C}_{i}^{\mathrm{pl}-\mathrm{bk}}\right)_{A} \rightarrow \mathcal{C}_{i}\right)
$$

such that every automorphism (of an object of $\mathcal{C}_{i}$ ) induced by $\phi$ is a Div-identity automorphism. Thus, since $\Psi$ preserves pull-back morphisms (cf. Theorem 3.4(iii)) and Dividentity automorphisms (cf. Theorem 4.2(i) and our assumption that the $\Phi_{i}$ are perf-factorial), we thus conclude that $\Psi$ preserves ' $\mathcal{O}^{\times}(-)$', as desired. This completes the proof of the existence and 1-uniqueness of a 1-commutative diagram as in the statement of assertion (i).

The rigidity assertion in the statement of assertion (i) follows by observing that if $\alpha \in \operatorname{Aut}\left(\mathcal{C}_{i} \rightarrow \mathcal{C}_{i}^{\text {un-tr }}\right.$ ), then every automorphism (of an object of $\mathcal{C}_{i}^{\text {un-tr) }}$ ) induced by $\alpha$ is a Div-identity automorphism. (Indeed, this follows by applying the functoriality of $\alpha$ to (coangular) pre-steps, in light of the second equivalence of categories of Definition 1.3(iii)(d).) In particular, it follows that if $A \in \mathrm{Ob}\left(\mathcal{C}_{i}\right), A_{\mathcal{D}} \stackrel{\text { def }}{=} \operatorname{Base}(A)$, then the element

$$
\alpha_{A} \in \operatorname{Aut}\left(\left(\mathcal{C}_{i}^{\mathrm{pl}-\mathrm{bk}}\right)_{A} \rightarrow \mathcal{D}_{i}\right) \stackrel{\sim}{\rightarrow} \operatorname{Aut}\left(\left(\mathcal{D}_{i}\right)_{A_{\mathcal{D}}} \rightarrow \mathcal{D}_{i}\right)
$$

determined by $\alpha$ maps (under composition with $\Phi_{i}: \mathcal{D}_{i} \rightarrow \mathfrak{M o n}$ ) to the identity element of $\operatorname{Aut}\left(\left(\mathcal{D}_{i}\right)_{A_{\mathcal{D}}} \rightarrow \mathfrak{M o n}\right)$. Thus, since $\mathcal{D}_{i}$ is Div-slim, it follows that every automorphism (of an object of $\mathcal{C}_{i}^{\text {un-tr }}$ ) induced by $\alpha$ is a base-identity automorphism, hence trivial (since $\mathcal{C}_{i}^{\text {un-tr }}$ is of unit-trivial type). This completes the proof of assertion (i).

Next, we consider assertion (ii). First, let us observe that we obtain a 1-commutative diagram

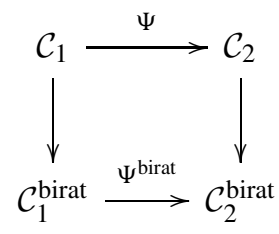

(cf. Corollary 4.10). Since, moreover, the base-isomorphisms of $\mathcal{C}_{i}^{\text {birat }}$ are precisely the morphisms of $\mathcal{C}_{i}^{\text {birat }}$ which are abstractly equivalent to morphisms that arise from base-isomorphisms of $\mathcal{C}_{i}$ (cf. Proposition 4.4(iv)), it follows that $\Psi^{\text {birat }}$ preserves baseisomorphisms, hence also pull-back morphisms (cf. Proposition 1.7(ii)). Thus, since $\mathcal{D}_{i}$ is Div-slim, the base-identity endomorphisms of $A \in \mathrm{Ob}\left(\mathcal{C}_{i}^{\text {birat }}\right)$ may be characterized as the endomorphisms of $A$ that arise from endomorphisms

$$
\phi \in \operatorname{End}\left(\left(\mathcal{C}_{i}^{\text {birat }}\right)_{A}^{\mathrm{pl}-\mathrm{bk}} \rightarrow \mathcal{C}_{i}^{\text {birat }}\right)
$$

such that every endomorphism (of an object of $\mathcal{C}_{i}^{\text {birat}}$ ) induced by $\phi$ projects to an automorphism of $\mathcal{D}_{i}$ that is mapped by $\Phi_{i}$ to an identity automorphism. Since, by Theorem 4.2(ii) (cf. also the fact that the $\Phi_{i}$ are perf-factorial and non-dilating), it follows immediately from the definition of $\mathcal{C}_{i}^{\text {birat }}$ that $\Psi^{\text {birat }}$ preserves those endomorphisms (of an object of $\mathcal{C}_{i}^{\text {birat }}$ ) that project to an automorphism of $\mathcal{D}_{i}$ that is mapped by $\Phi_{i}$ to an identity automorphism, we thus conclude that $\Psi^{\text {birat }}$ preserves the base-identity endomorphisms (hence, in particular, that $\Psi^{\text {birat }}$ preserves ' $\mathcal{O}^{\times}(-)$'). Thus, we obtain a 1-commutative 
diagram

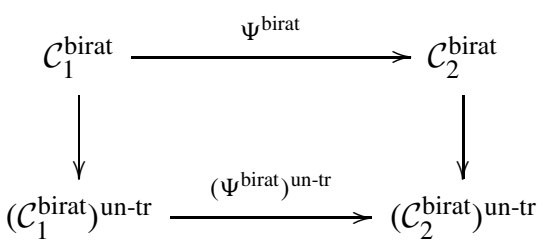

(where the vertical arrows are the natural functors and the horizontal arrows are equivalences of categories). Since, moreover, the Frobenioids $\left(\mathcal{C}_{i}^{\text {birat }}\right)^{\text {un-tr }}$ are of isotropic, unit-trivial and group-like type, we thus conclude that we obtain a 1-commutative diagram

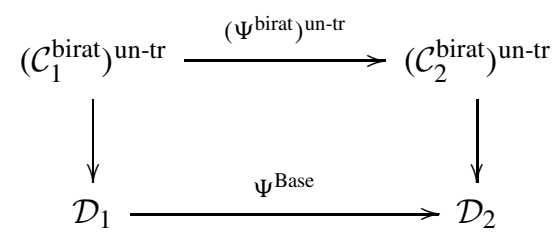

(cf. Proposition 3.11(iii)). Thus, by composing diagrams, we obtain a 1-commutative diagram as in the statement of assertion (ii), which is easily verified to be 1-unique. Finally, the rigidity assertion in the statement of assertion (ii) follows from Proposition 1.13(i). This completes the proof of assertion (ii).

Next, we observe that assertion (iii) follows formally from assertion (ii) and Theorem 4.9 (cf. also Definition 1.3(i)(a), (b) and the technique of using the equivalence of categories ' $\mathcal{D}^{*} \stackrel{\sim}{\rightarrow} \mathcal{D}$ ' applied in Proposition 2.2(ii)).

Finally, we consider assertion (iv). In light of the structure of an elementary Frobenioid (cf. Definition 1.1(iii)), the existence of a 1-commutative diagram as in the statement of assertion (iv) now follows simply by concatenating assertions (ii) and (iii) with the fact that $\Psi$ preserves Frobenius degrees (cf. Theorem 3.4(iii)). Finally, the rigidity assertion follows via the same argument as was applied to prove the rigidity assertion that appears in the statement of assertion (i). This completes the proof of assertion (iv).

Remark 4.11.1. Note that since 'slim always implies Div-slim', it follows that, at least when the divisorial monoids involved are perf-factorial, Corollary 4.11(ii) constitutes a substantial strengthening of Theorem 3.4(v).

Remark 4.11.2. Observe that in Example 3.9, since the subgroup $U$ of $G=\operatorname{Aut}\left(\mathcal{D}_{A} \rightarrow \mathcal{D}\right)$ (where $A \in \operatorname{Ob}(\mathcal{D})$ ) acts trivially on $V \times W$, it follows that $\mathcal{D}$ fails to be Div-slim, so the non-preservation of units that occurs in this example does not contradict Corollary 4.11(i) and (ii). In a similar vein, in Example 3.10, since $G=\operatorname{Aut}\left(\mathcal{D}_{A} \rightarrow \mathcal{D}\right)($ where $A \in \operatorname{Ob}(\mathcal{D})$ ) acts trivially on $\mathbb{Z}_{\geq 0}$, it follows that $\mathcal{D}$ fails to be Div-slim, so the non-preservation of baseidentity endomorphisms that occurs in this example does not contradict Corollary 4.11(ii).

COROLLARY 4.12. (Category-theoreticity of the functor to an elementary Frobenioid II) For $i=1,2$, let $\Phi_{i}$ be a divisorial monoid on a connected, totally epimorphic category $\mathcal{D}_{i}$ which is Frobenius-slim, let $\mathcal{C}_{i} \rightarrow \mathbb{F}_{\Phi_{i}}$ be a Frobenioid of rationally standard type, let $0_{\mathcal{D}_{i}}$ be the monoid on $\mathcal{D}_{i}$ that assigns to every object of $\mathcal{D}_{i}$ the monoid with one element and let

$$
\Psi: \mathcal{C}_{1} \stackrel{\sim}{\rightarrow} \mathcal{C}_{2}
$$


be an equivalence of categories. If $\mathcal{C}_{1}, \mathcal{C}_{2}$ are of group-like type, then we also assume that both $\Psi$ and some quasi-inverse to $\Psi$ preserve base-isomorphisms. Then there exists a 1unique functor $\Psi^{0}: \mathbb{F}_{0_{\mathcal{D}_{1}}} \rightarrow \mathbb{F}_{0_{\mathcal{D}_{2}}}$ that fits into a 1-commutative diagram

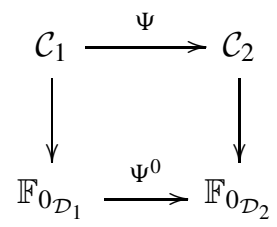

(where the vertical arrows are the natural projection functors, determined by the Frobenius degree and the projection to $\mathcal{D}_{i}$ (cf. Proposition 4.4(i)) and the horizontal arrows are equivalences of categories). Moreover, if $\mathcal{D}_{1}, \mathcal{D}_{2}$ are slim, then each of the composite functors of this diagram is rigid.

Proof. First, we observe (cf. Theorem 3.4(i)) that we may assume without loss of generality that $\mathcal{C}_{1}, \mathcal{C}_{2}$ are of isotropic type (cf. Remark 4.5.1). Now the natural projection functors $\mathcal{C}_{i} \rightarrow \mathbb{F}_{0_{\mathcal{D}_{i}}}$ may be identified with the natural functors $\mathcal{C}_{i} \rightarrow \mathcal{C}_{i}^{\text {birat }} \rightarrow\left(\mathcal{C}_{i}^{\text {birat }}\right)^{\text {un-tr }}$ (cf. Proposition 3.11(i)). In particular, if $\mathcal{C}_{1}, \mathcal{C}_{2}$ are of group-like type (cf. Theorem 3.4(ii)), then (since $\mathcal{C}_{i} \stackrel{\sim}{\rightarrow} \mathcal{C}_{i}^{\text {birat }}$ ) Corollary 4.12 follows from Theorem 3.4(iv). Thus, we may assume without loss of generality that $\mathcal{C}_{1}, \mathcal{C}_{2}$ are not of group-like type. Then Corollary 4.12 follows by applying Corollary 4.10 to pass from $\mathcal{C}_{i}$ to $\mathcal{C}_{i}^{\text {birat }}$ (where we note that, by Proposition 4.4(iv) and Theorem 3.4(iii), it follows that the resulting equivalence of categories $\Psi^{\text {birat }}$ preserves base-isomorphisms), followed by Theorem 3.4(iv) (where we note that, by Proposition 4.8(iii), $\mathcal{C}_{i}^{\text {birat }}$ is of standard type), which allows us to pass from $\mathcal{C}_{i}^{\text {birat }}$ to $\left(\mathcal{C}_{i}^{\text {birat }}\right)^{\text {un-tr }}$, as desired. Finally, the rigidity assertion follows from Proposition 1.13(i). This completes the proof of Corollary 4.12 .

Remark 4.12.1. One verifies immediately that if one takes the group $G$ of Example 3.10 to be residually finite, then the Frobenioid of Example 3.10 is of rationally standard and unit-trivial type (but not of group-like type) over a Frobenius-slim base category (which is not Div-slim cf. Remark 4.11.2). In particular, one may apply Corollary 4.12 to the self-equivalence of categories of Example 3.10. On the other hand, since this self-equivalence fails to preserve base-identity endomorphisms of Frobenius type, it follows that it is not possible to replace the ' $F_{0_{\mathcal{D}_{i}}}$ ' in the diagram of Corollary 4.12 by ' $\mathcal{D}_{i}$ '.

\section{Model Frobenioids}

In this section, we study the extent to which an arbitrary Frobenioid of isotropic type may be constructed explicitly as a 'model Frobenioid'. This study of 'model Frobenioids' will be of use in the consideration of the concrete examples of Frobenioids that we discuss in Section 6 below.

In the following discussion, we maintain the notation of Sections 1-4. In particular, we assume that we have been given a divisorial monoid $\Phi$ on a connected, totally epimorphic category $\mathcal{D}$ and a Frobenioid $\mathcal{C} \rightarrow \mathbb{F}_{\Phi}$. 
THeOREM 5.1. (Divisorial descriptions) Suppose that the Frobenioid $\mathcal{C}$ is of isotropic type. Let $A, A^{\prime} \in \mathrm{Ob}(\mathcal{C})$ be Frobenius-trivial, $A_{\mathcal{D}} \stackrel{\text { def }}{=} \operatorname{Base}(A) \in \mathrm{Ob}(\mathcal{D}) ; A_{\mathcal{D}}^{\prime} \stackrel{\text { def }}{=} \operatorname{Base}\left(A^{\prime}\right) \in$ $\mathrm{Ob}(\mathcal{D})$, let $\mathcal{D}^{\mathrm{isom}} \subseteq \mathcal{D}$ be the subcategory determined by the isomorphisms of $\mathcal{D}$ and let $\mathcal{D}_{D}^{\text {isom }} \stackrel{\text { def }}{=}\left(\mathcal{D}^{\text {isom }}\right)_{D}\left(\right.$ for $\left.D \in \mathrm{Ob}(\mathcal{D})=\mathrm{Ob}\left(\mathcal{D}^{\text {isom }}\right)\right)$. Write

$$
\operatorname{Pic}_{\Phi}(A) \stackrel{\text { def }}{=} \Phi^{\mathrm{gp}}(A) / \Phi^{\text {birat }}(A)
$$

(cf. Proposition 4.4(iii)) and $\operatorname{Pic}_{\mathcal{C}}(A)$ for the set of isomorphism classes of $\mathcal{C} \times \mathcal{D} \mathcal{D}_{A_{\mathcal{D}}}^{\text {isom }}$ (where the fiber product category is taken with respect to the natural functors $\mathcal{C} \rightarrow \mathcal{D}, \mathcal{D}_{A_{\mathcal{D}}}^{\text {isom }} \rightarrow \mathcal{D}-$ cf. Section 0). Then we have the following.

(i) The assignment that maps a pair of pre-steps

$$
(\phi: B \rightarrow A, \psi: B \rightarrow C)
$$

to the object

$$
\left(C, \operatorname{Base}(\phi) \circ \operatorname{Base}(\psi)^{-1}\right) \in \mathrm{Ob}\left(\mathcal{C} \times_{\mathcal{D}} \mathcal{D}_{A_{\mathcal{D}}}^{\text {isom }}\right)
$$

on the one hand and to the element

$$
\Phi(\phi)^{-1}(\operatorname{Div}(\psi)-\operatorname{Div}(\phi)) \in \Phi^{\mathrm{gp}}(A)
$$

on the other hand determines a bijection $\operatorname{Pic}_{\Phi}(A) \stackrel{\sim}{\rightarrow} \operatorname{Pic}_{\mathcal{C}}(A)$. Moreover, if $(C, \zeta$ : $\left.C_{\mathcal{D}} \stackrel{\sim}{\rightarrow} A_{\mathcal{D}}\right) \in \mathrm{Ob}\left(\mathcal{C} \times_{\mathcal{D}} \mathcal{D}_{A_{\mathcal{D}}}^{\text {isom }}\right)$ (where $C \in \mathrm{Ob}(\mathcal{C})$ and $C_{\mathcal{D}} \stackrel{\text { def }}{=} \operatorname{Base}(C)$ ) corresponds, via this bijection, to an element $\gamma \in \operatorname{Pic}_{\Phi}(A)$ and $\kappa: C \rightarrow C^{\prime}$ is a morphism of Frobenius type, then $\left(C^{\prime}, \zeta \circ \operatorname{Base}(\kappa)^{-1}\right) \in \mathrm{Ob}\left(\mathcal{C} \times{ }_{\mathcal{D}} \mathcal{D}_{A_{\mathcal{D}}}^{\text {isom }}\right)$ corresponds to the element $\operatorname{deg}_{\mathrm{Fr}}(\kappa) \cdot \gamma \in \operatorname{Pic}_{\Phi}(A)$.

(ii) If

$$
\left(B, \lambda: B_{\mathcal{D}} \stackrel{\sim}{\rightarrow} A_{\mathcal{D}}\right) \in \mathrm{Ob}\left(\mathcal{C} \times \times_{\mathcal{D}} \mathcal{D}_{A_{\mathcal{D}}}^{\text {isom }}\right), \quad\left(B^{\prime}, \lambda^{\prime}: B_{\mathcal{D}}^{\prime} \stackrel{\sim}{\rightarrow} A_{\mathcal{D}}^{\prime}\right) \in \mathrm{Ob}\left(\mathcal{C} \times \mathcal{D} \mathcal{D}_{A_{\mathcal{D}}^{\prime}}^{\text {isom }}\right)
$$

(where $B_{\mathcal{D}} \stackrel{\text { def }}{=} \operatorname{Base}(B)$ and $B_{\mathcal{D}}^{\prime} \stackrel{\text { def }}{=} \operatorname{Base}\left(B^{\prime}\right)$ ), then there exists a morphism

$$
\phi: B \rightarrow B^{\prime}
$$

in $\mathcal{C}$ of Frobenius degree $d$ such that $\operatorname{Base}(\phi)=\left(\lambda^{\prime}\right)^{-1} \circ \theta \circ \lambda$, where $\theta: A_{\mathcal{D}} \rightarrow A_{\mathcal{D}}^{\prime}$ is a morphism of $\mathcal{D}$, and $\operatorname{Div}(\phi)=z \in \Phi(B)$ if and only if the classes $\beta \in \operatorname{Pic}_{\Phi}(A)$, $\beta^{\prime} \in \operatorname{Pic}_{\Phi}\left(A^{\prime}\right)$ determined by $B, B^{\prime}$, respectively, via the bijection of (i) satisfy the relation

$$
d \cdot \beta+\left.z\right|_{A_{\mathcal{D}}}=(\Phi(\theta))\left(\beta^{\prime}\right) \in \operatorname{Pic}_{\Phi}(A)
$$

(where, by abuse of notation, we denote by $\left.z\right|_{A_{\mathcal{D}}}$ the image of $\Phi(\lambda)^{-1}(z) \in \Phi(A)$ in $\left.\operatorname{Pic}_{\Phi}(A)\right)$. Moreover, if such a morphism exists, then its unit-equivalence class (i.e., its image in $\mathcal{C}^{\text {un-tr }}$ or, equivalently, $\mathbb{F}_{\Phi}-c f$. Proposition 3.3(iv)) is unique.

(iii) The subcategory

$$
\mathcal{C}^{\mathrm{Fr}-\operatorname{tr}} \subseteq \mathcal{C}
$$

determined by the Frobenius-trivial objects and isometric morphisms is a Frobenioid of isotropic, group-like, base-trivial and Aut-ample type. In particular, the isomorphism class of a Frobenius-trivial object of $\mathcal{C}$ is completely determined by the isomorphism class of its projection to $\mathcal{D}$ and all Frobenius-trivial objects of $\mathcal{C}$ are Aut-ample. 
(iv) Suppose that $\mathcal{C}$ is of unit-trivial type. Then any skeletal subcategory $\mathcal{P} \subseteq\left(\mathcal{C}^{\text {Fr-tr }}\right)^{\mathrm{pl}-\mathrm{bk}}$ determines $a$ base-section of $\mathcal{C}$ and any base-section of $\mathcal{C}$ admits an associated Frobenius-section $\mathcal{F}$. Moreover, $\mathcal{C}$ is of model type.

Proof. First, we consider assertion (i). Let us refer to a(n) (ordered) pair of pre-steps as an Apair if the first pre-step has codomain $A$ and the second pre-step has the same domain as the first. Let us say that two $A$-pairs $(\phi: B \rightarrow A, \psi: B \rightarrow C),\left(\phi^{\prime}: B^{\prime} \rightarrow A, \psi: B^{\prime} \rightarrow C^{\prime}\right)$ are isomorphic if there exist isomorphisms $\iota_{B}: B \stackrel{\sim}{\rightarrow} B^{\prime}, \iota_{C}: C \stackrel{\sim}{\rightarrow} C^{\prime}$ such that $\phi^{\prime} \circ \iota_{B}=\phi, \psi^{\prime} \circ$ $\iota_{B}=\iota_{C} \circ \psi$. Then observe that by the equivalences of categories of Definition 1.3(iii)(d), it follows that the assignment

$$
(\phi: B \rightarrow A, \psi: B \rightarrow C) \mapsto\left(\Phi(\phi)^{-1}(\operatorname{Div}(\phi)), \Phi(\phi)^{-1}(\operatorname{Div}(\psi))\right) \in \Phi(A) \times \Phi(A)
$$

determines a bijection from the set of isomorphism classes of $A$-pairs onto $\Phi(A) \times \Phi(A)$; in particular, we obtain a map $\Phi(A) \times \Phi(A) \rightarrow \operatorname{Pic}_{\mathcal{C}}(A)$. Moreover, relative to this bijection, replacing an element $(x, y) \in \Phi(A) \times \Phi(A)$ by an element $(x+z, y+z) \in \Phi(A) \times \Phi(A)$ (where $z \in \Phi(A))$ corresponds to replacing $(\phi: B \rightarrow A, \psi: B \rightarrow C)$ by $(\phi \circ \delta, \psi \circ \delta)$, for some pre-step $\delta$; in particular, such replacements do not affect the element of $\operatorname{Pic}_{\mathcal{C}}(A)$ determined by the $A$-pair.

Now I claim that the map $\Phi(A) \times \Phi(A) \rightarrow \operatorname{Pic}_{\mathcal{C}}(A)$ of the above discussion factors through $\operatorname{Pic}_{\Phi}(A)$. Indeed, suppose that $(x, y) \in \Phi(A) \times \Phi(A),\left(x^{\prime}, y^{\prime}\right) \in \Phi(A) \times \Phi(A)$ map to the same element of $\operatorname{Pic}_{\Phi}(A)$. Then, by the definition of ' $\Phi^{\text {birat }}(A)$ ' (cf. the statements and proofs of Proposition 4.4(i), (iii)), it follows that there exists a pair of base-equivalent presteps $\delta_{1}, \delta_{2}: D \rightarrow A$ such that

$$
\Phi\left(\delta_{1}\right)^{-1}\left(\operatorname{Div}\left(\delta_{1}\right)\right)+x^{\prime}+y+z=\Phi\left(\delta_{2}\right)^{-1}\left(\operatorname{Div}\left(\delta_{2}\right)\right)+x+y^{\prime}+z
$$

for some $z \in \Phi(A)$ (cf. also the definition of 'gp' in Section 0). Thus, by replacing $\delta_{1}, \delta_{2}$ by the composite of $\delta_{1}, \delta_{2}$ with an appropriate pre-step (cf. Definition 1.3(iii)(d)), we may assume that

$$
\Phi\left(\delta_{1}\right)^{-1}\left(\operatorname{Div}\left(\delta_{1}\right)\right)=x+y^{\prime}+z^{\prime}, \quad \Phi\left(\delta_{2}\right)^{-1}\left(\operatorname{Div}\left(\delta_{2}\right)\right)=x^{\prime}+y+z^{\prime}
$$

for some $z^{\prime} \in \Phi(A)$ (for instance, one natural choice for $z^{\prime}$ is $\Phi\left(\delta_{1}\right)^{-1}\left(\operatorname{Div}\left(\delta_{1}\right)\right)+$ $\left.x^{\prime}+y+z=\Phi\left(\delta_{2}\right)^{-1}\left(\operatorname{Div}\left(\delta_{2}\right)\right)+x+y^{\prime}+z\right)$ and by replacing $(x, y)$ by $\left(x+z^{\prime}, y+z^{\prime}\right)$ (cf. discussion of the preceding paragraph), it follows that we may assume, without loss of generality, that $z^{\prime}=0$. Next, by applying the first equivalence of categories of Definition 1.3(iii)(d), we observe that there exists a pre-step $\delta^{\dagger}: D \rightarrow D^{\dagger}$ such that $\operatorname{Div}\left(\delta^{\dagger}\right)=$ $\Phi\left(\delta_{i}\right)\left(x+x^{\prime}+y+y^{\prime}\right)$, where $i=1,2$ (and we note that $\Phi\left(\delta_{i}\right)$ is independent of $i$, since $\delta_{1}$, $\delta_{2}$ are base-equivalent). Thus, (again by Definition 1.3(iii)(d)) we conclude that there exist base-equivalent pre-steps $\delta_{1}^{A}, \delta_{2}^{A}: A \rightarrow D^{\dagger}$ such that $\delta^{\dagger}=\delta_{2}^{A} \circ \delta_{1}=\delta_{1}^{A} \circ \delta_{2}$. In particular, we have $\operatorname{Div}\left(\delta_{1}^{A}\right)=x+y^{\prime}, \operatorname{Div}\left(\delta_{2}^{A}\right)=x^{\prime}+y$.

Let $\epsilon: E \rightarrow A$ be a pre-step with $\Phi(\epsilon)^{-1}(\operatorname{Div}(\epsilon))=x+x^{\prime}$ (cf. Definition 1.3(iii)(d)), let $(\phi: B \rightarrow A, \psi: B \rightarrow C)$ be an $A$-pair that corresponds to $(x, y)$ and let $\left(\phi^{\prime}: B^{\prime} \rightarrow A, \psi^{\prime}\right.$ : $B^{\prime} \rightarrow C^{\prime}$ ) be an $A$-pair that corresponds to $\left(x^{\prime}, y^{\prime}\right)$. Then since $x, x^{\prime} \leq x+x^{\prime}$, it follows (cf. Definition 1.3(iii)(d)) that there exist factorizations $\epsilon=\phi \circ \eta, \epsilon=\phi^{\prime} \circ \eta^{\prime}$, where $\eta: E \rightarrow B$, $\eta^{\prime}: E \rightarrow B^{\prime}$ are pre-steps. Moreover, by applying the second equivalence of categories of Definition 1.3(iii)(d), to $D^{\dagger}$, we conclude from the existence of the composites of $\epsilon: E \rightarrow A$ 
with $\delta_{1}^{A}, \delta_{2}^{A}: A \rightarrow D^{\dagger}$ that there exists a pre-step $\epsilon^{F}: F \rightarrow D^{\dagger}$ and a pair of base-equivalent pre-steps $\delta_{1}^{E}, \delta_{2}^{E}: E \rightarrow F$ such that the following relations hold:

$$
\begin{array}{cl}
\epsilon^{F} \circ \delta_{1}^{E}=\delta_{1}^{A} \circ \epsilon, & \epsilon^{F} \circ \delta_{2}^{E}=\delta_{2}^{A} \circ \epsilon \\
\operatorname{Div}\left(\delta_{1}^{E}\right)=(\Phi(\epsilon))\left(x+y^{\prime}\right), & \operatorname{Div}\left(\delta_{2}^{E}\right)=(\Phi(\epsilon))\left(x^{\prime}+y\right)
\end{array}
$$

(so $\Phi\left(\epsilon^{F}\right)^{-1}\left(\operatorname{Div}\left(\epsilon^{F}\right)\right)=\Phi\left(\delta_{i}^{A}\right)^{-1}\left(x+x^{\prime}\right)$, for $\left.i=1,2\right)$. On the other hand, since $\operatorname{Div}(\psi \circ$ $\eta)=(\Phi(\epsilon))\left(x^{\prime}+y\right)=\operatorname{Div}\left(\delta_{2}^{E}\right), \operatorname{Div}\left(\psi^{\prime} \circ \eta^{\prime}\right)=(\Phi(\epsilon))\left(x+y^{\prime}\right)=\operatorname{Div}\left(\delta_{1}^{E}\right)$, we thus conclude (cf. Definition 1.3(iii)(d) applied to the pairs of pre-steps $\left(\psi \circ \eta: E \rightarrow C, \delta_{2}^{E}: E \rightarrow F\right.$ ) and $\left(\psi^{\prime} \circ \eta^{\prime}: E \rightarrow C^{\prime}, \delta_{1}^{E}: E \rightarrow F\right)$ emanating from $E$ ) that there exists an isomorphism $\iota$ : $C \stackrel{\sim}{\rightarrow} C^{\prime}$ such that $\operatorname{Base}\left(\psi^{\prime} \circ \eta^{\prime}\right)=\operatorname{Base}(\iota \circ \psi \circ \eta)$, $\operatorname{Base}(\iota \circ \psi) \circ \operatorname{Base}(\phi)^{-1}=\operatorname{Base}\left(\psi^{\prime}\right) \circ$ $\operatorname{Base}\left(\phi^{\prime}\right)^{-1}$. That is to say, we have a (not necessarily commutative!) diagram of pre-steps

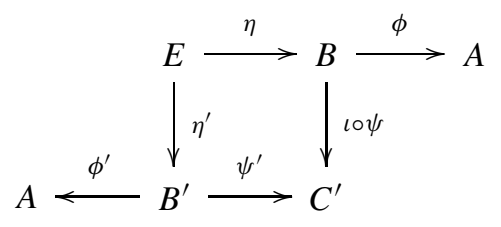

whose projection to $\mathcal{D}$ is a commutative diagram of isomorphisms that is compatible with identification of the two copies of $A_{\mathcal{D}}$. In particular, we conclude that $(C, \operatorname{Base}(\phi) \circ$ $\left.\operatorname{Base}(\psi)^{-1}\right)$ and $\left(C^{\prime}, \operatorname{Base}\left(\phi^{\prime}\right) \circ \operatorname{Base}\left(\psi^{\prime}\right)^{-1}\right)$ determine the same element of $\operatorname{Pic}_{\mathcal{C}}(A)$. This completes the proof of the claim.

Thus, we obtain a map $\operatorname{Pic}_{\Phi}(A) \rightarrow \operatorname{Pic}_{C}(A)$. It follows immediately from Definition 1.3(i)(b), that this map is a surjection. To show that this map is injective, it suffices to consider $(x, y) \in \Phi(A) \times \Phi(A)$ and $\left(x^{\prime}, y^{\prime}\right) \in \Phi(A) \times \Phi(A)$ that map to the same element of $\operatorname{Pic}_{\mathcal{C}}(A)$. Let $(\phi: B \rightarrow A, \psi: B \rightarrow C)$ be an $A$-pair that corresponds to $(x, y)$ and let $\left(\phi^{\prime}: B^{\prime} \rightarrow A, \psi: B^{\prime} \rightarrow C\right)$ be an $A$-pair that corresponds to $\left(x^{\prime}, y^{\prime}\right)$. By our assumption that $(x, y)$ and $\left(x^{\prime}, y^{\prime}\right)$ map to the same element of $\operatorname{Pic}_{\mathcal{C}}(A)$, it follows that we may assume that $\operatorname{Base}\left(\phi^{\prime}\right) \circ \operatorname{Base}\left(\psi^{\prime}\right)^{-1}=\operatorname{Base}(\phi) \circ \operatorname{Base}(\psi)^{-1}$. Thus, by applying Definition 1.3(iii)(d) we obtain a (not necessarily commutative!) diagram of pre-steps

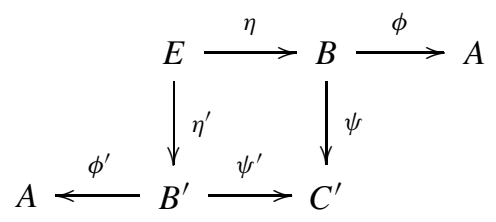

such that $\phi \circ \eta=\phi^{\prime} \circ \eta^{\prime}$ and whose projection to $\mathcal{D}$ is a commutative diagram of isomorphisms that is compatible with identification of the two copies of $A_{\mathcal{D}}$. Thus, it follows that $\psi \circ \eta, \psi^{\prime} \circ \eta^{\prime}: E \rightarrow C$ are base-equivalent, hence determine an element of $\Phi^{\text {birat }}(C)$, which may be transported via $\psi, \phi$ (or, equivalently, $\psi^{\prime}, \phi^{\prime}$ ) to an element of $\Phi^{\text {birat }}(A) \subseteq \Phi^{\mathrm{gp}}(A)$ which (cf. the discussion of the preceding paragraph) is easily verified to be $x+y^{\prime}-x^{\prime}-y \in \Phi^{\mathrm{gp}}(A)$. This completes the proof of the injectivity, hence also of the bijectivity of the map $\operatorname{Pic}_{\Phi}(A) \rightarrow \operatorname{Pic}_{C}(A)$. Also, the portion of assertion (i) concerning morphisms of Frobenius type follows easily by considering commutative diagrams as in Proposition 1.10(i). This completes the proof of assertion (i). 
Now assertion (ii) follows formally from assertion (i) (cf. also Remark 1.1.1, the factorization of Definition 1.3(iv)(a) and the faithfulness portion of Proposition 3.3(iv)).

Next, we consider assertion (iii). First, let us observe that by assertion (i), any isomorphism $\alpha_{\mathcal{D}}: A_{\mathcal{D}}^{\prime} \stackrel{\sim}{\rightarrow} A_{\mathcal{D}}$ determines an object $\left(A^{\prime}, \alpha\right) \in \mathrm{Ob}\left(\mathcal{C} \times_{\mathcal{D}} \mathcal{D}_{A_{\mathcal{D}}}^{\text {isom }}\right)$ which (in light of the fact that $A^{\prime}$ is Frobenius-trivial, hence admits base-identity endomorphisms of Frobenius type of arbitrary prescribed Frobenius degree) corresponds (via the bijection of assertion (i)) to an element $\xi \in \operatorname{Pic}_{\Phi}(A)$ such that $d \cdot \xi=\xi$, for all $d \in \mathbb{N}_{\geq 1}$. Thus, taking $d=2$ implies that $\xi=0$, i.e., (cf. the definition of $\operatorname{Pic}_{\mathcal{C}}(A)$ ) that there exists an isomorphism $\alpha: A^{\prime} \stackrel{\sim}{\rightarrow} A$ such that $\alpha_{\mathcal{D}}=\operatorname{Base}(\alpha)$. In particular, we conclude that baseisomorphic Frobenius-trivial objects of $\mathcal{C}$ are, in fact, isomorphic and that all Frobeniustrivial objects of $\mathcal{C}$ are Aut-ample. In light of these observations, it follows immediately that $\mathcal{C}^{\mathrm{Fr}-\mathrm{tr}}$ satisfies the conditions of Definition 1.3, i.e., that $\mathcal{C}^{\mathrm{Fr}-\mathrm{tr}}$ is a Frobenioid (of isotropic, group-like, base-trivial and Aut-ample type). This completes the proof of assertion (iii).

Finally, we consider assertion (iv). First, we observe that since $\mathcal{C}$ is of unit-trivial type, it follows immediately (cf., e.g., Proposition 3.3(iii), (iv)) that given any two objects $A, B \in \mathrm{Ob}(\mathcal{C})$, a pull-back morphism $A \rightarrow B$ (respectively, base-identity endomorphism of Frobenius type of $A$ ) is uniquely determined by its projection to $\mathcal{D}$ (respectively, by its Frobenius degree). Moreover, by assertion (iii), it follows immediately that if $A, B \in$ $\mathrm{Ob}\left(\mathcal{C}^{\mathrm{Fr}-\mathrm{tr}}\right)$, then any morphism $\operatorname{Base}(A) \rightarrow \operatorname{Base}(B)$ (in $\mathcal{D}$ ) lifts to a pull-back morphism of $\mathcal{C}^{\text {Fr-tr }}$. Thus, we conclude that the natural projection functor

$$
\left(\mathcal{C}^{\mathrm{Fr}-\mathrm{tr}}\right)^{\mathrm{pl-bk}} \rightarrow \mathcal{D}
$$

is an equivalence of categories, hence that any skeletal subcategory $\mathcal{P} \subseteq\left(\mathcal{C}^{\text {Fr-tr }}\right)^{\mathrm{pl}-\mathrm{bk}}$ determines a base-section of $\mathcal{C}$, and that any base-section of $\mathcal{C}$ admits an associated Frobeniussection. Moreover, since $\mathcal{C}$ is of unit-trivial type, it follows immediately from the structure of an elementary Frobenioid (cf. the description of the kernel in Proposition 4.4(iii)) that $\mathcal{C}$ is of birationally Frobenius-normalized type, hence also of model type, as desired. This completes the proof of assertion (iv).

The explicit descriptions of Theorem 5.1(i) and (ii) motivate the following construction/result.

THEOREM 5.2. (Model Frobenioids) Let $\Phi: \mathcal{D} \rightarrow$ Mon be a divisorial monoid on $\mathcal{D}$, let $\mathbb{B}: \mathcal{D} \rightarrow \mathfrak{M o n}$ be a group-like monoid on $\mathcal{D}$ and let $\operatorname{Div}_{\mathbb{B}}: \mathbb{B} \rightarrow \underline{\Phi}^{\mathrm{gp}}$ be a homomorphism of monoids on $\mathcal{D}$. Denote the group-like monoid determined by the image of $\operatorname{Div}_{\mathbb{B}}$ by $\underline{\Phi}^{\text {birat }} \subseteq \Phi^{\mathrm{gp}}$. Then we have the following.

(i) A well-defined category $\underline{\mathcal{C}}$ may be constructed in the following fashion. The objects of $\underline{\mathcal{C}}$ are pairs of the form

$$
\left(A_{\mathcal{D}}, \alpha\right)
$$

where $A_{\mathcal{D}} \in \mathrm{Ob}(\mathcal{D}), \alpha \in \underline{\Phi}\left(A_{\mathcal{D}}\right)^{\mathrm{gp}}$. Set $\operatorname{Base}(A) \stackrel{\text { def }}{=} A_{\mathcal{D}}, \underline{\Phi}(A) \stackrel{\text { def }}{=} \underline{\Phi}\left(A_{\mathcal{D}}\right)$ and $\mathbb{B}(A) \stackrel{\text { def }}{=}$ $\mathbb{B}\left(A_{\mathcal{D}}\right)$. $A$ morphism

$$
\phi: A \stackrel{\text { def }}{=}\left(A_{\mathcal{D}}, \alpha\right) \rightarrow B \stackrel{\text { def }}{=}\left(B_{\mathcal{D}}, \beta\right)
$$

(where $A_{\mathcal{D}}, B_{\mathcal{D}} \in \mathrm{Ob}(\mathcal{D}), \alpha \in \underline{\Phi}(A)^{\mathrm{gp}}$ and $\left.\beta \in \underline{\Phi}(B)^{\mathrm{gp}}\right)$ is defined to be a collection of data as follows: (a) an element $\operatorname{deg}_{\mathrm{Fr}}(\phi) \in \overline{\mathbb{N}}_{\geq 1}$, which we shall refer to as the 
Frobenius degree of $\phi$; (b) a morphism $\operatorname{Base}(\phi): A_{\mathcal{D}} \rightarrow B_{\mathcal{D}}$, which we shall refer to as the projection to $\mathcal{D}$ to $\phi$; (c) an element $\operatorname{Div}(\phi) \in \Phi(A)$, which we shall refer to as the zero divisor of $\phi$; (d) an element $u_{\phi} \in \mathbb{B}(A)$ whose image $\operatorname{Div}_{\mathbb{B}}\left(u_{\phi}\right) \in \underline{\Phi}(A)^{\mathrm{gp}}$ satisfies the relation

$$
\operatorname{deg}_{\mathrm{Fr}}(\phi) \cdot \alpha+\operatorname{Div}(\phi)=(\underline{\Phi}(\operatorname{Base}(\phi)))(\beta)+\operatorname{Div}_{\mathbb{B}}\left(u_{\phi}\right)
$$

in $\underline{\Phi}(A)^{\mathrm{gp}}$. The composite $\psi \circ \phi$ of two morphisms

$$
\begin{aligned}
& \phi=\left(\operatorname{deg}_{\mathrm{Fr}}(\phi), \operatorname{Base}(\phi), \operatorname{Div}(\phi), u_{\phi}\right): A \rightarrow B, \\
& \psi=\left(\operatorname{deg}_{\mathrm{Fr}}(\psi), \operatorname{Base}(\psi), \operatorname{Div}(\psi), u_{\psi}\right): B \rightarrow C
\end{aligned}
$$

is defined as

$$
\begin{gathered}
\psi \circ \phi \stackrel{\text { def }}{=}\left(\operatorname{deg}_{\mathrm{Fr}}(\psi) \cdot \operatorname{deg}_{\mathrm{Fr}}(\phi), \operatorname{Base}(\psi) \circ \operatorname{Base}(\phi),(\underline{\Phi}(\operatorname{Base}(\phi)))(\operatorname{Div}(\psi))\right. \\
\left.+\operatorname{deg}_{\mathrm{Fr}}(\psi) \cdot \operatorname{Div}(\phi),(\mathbb{B}(\operatorname{Base}(\phi)))\left(u_{\psi}\right)+\operatorname{deg}_{\mathrm{Fr}}(\psi) \cdot u_{\phi}\right)
\end{gathered}
$$

(cf. Remark 1.1.1). Moreover, the Frobenius degree, projection to $\mathcal{D}$ and zero divisor determine a functor $\underline{\mathcal{C}} \rightarrow \mathbb{F}_{\Phi}$.

(ii) The category $\underline{\mathcal{C}}$ is a Frobenioid (with respect to the functor $\underline{\mathcal{C}} \rightarrow \mathbb{F}_{\Phi}$ ) of isotropic and model - hence, in particular, birationally Frobenius-normalized - type. We shall refer to $\underline{\mathcal{C}}$ as the model Frobenioid defined by the divisor monoid $\underline{\Phi}$ and the rational function monoid $\mathbb{B}$ (which we regard as equipped with the homomorphism $\operatorname{Div}_{\mathbb{B}}: \mathbb{B} \rightarrow \Phi^{\mathrm{gp}}$ ). Moreover, there is a natural isomorphism of functors between the functor ' $\mathrm{O}^{\times}(-)$' on $\mathcal{D}$ associated to the Frobenioid $\underline{\mathcal{C}}^{\text {birat }}$ (cf. Propositions 2.2(ii), (iii) and 4.4(ii)) and the functor $\mathbb{B} ;$ this isomorphism is compatible with the homomorphisms $\mathcal{O}^{\times}(-) \rightarrow \Phi^{\mathrm{gp}}$ (cf. Proposition 4.4(iii)), $\operatorname{Div}_{\mathbb{B}}: \mathbb{B} \rightarrow \underline{\Phi}^{\mathrm{gp}}$.

(iii) $\underline{\mathcal{C}}$ is of standard type if and only if the following conditions are satisfied: (a) if $\underline{\Phi}$ is the zero monoid, then $\underline{\mathcal{C}}$ admits a Frobenius-compact object; (b) $\mathcal{D}$ is of FSMFF-type; (c) $\Phi$ is non-dilating. $\underline{\mathcal{C}}$ is of rationally standard type if and only if the following conditions are satisfied: (a) $\underline{\mathcal{C}}$ is of rational and standard type; (b) $\left(\underline{\mathcal{C}}^{\text {un-tr }}\right)^{\text {birat }}$ admits a Frobeniuscompact object.

(iv) Suppose that $\Phi=\Phi, \mathbb{B}$ is the rational function monoid on $\mathcal{D}$ associated to the Frobenioid $\mathcal{C}$ (cf. Proposition 4.4(ii)), Div $\mathbb{B}: \mathbb{B} \rightarrow \Phi^{\mathrm{gp}}$ is the natural homomorphism $\mathcal{O}^{\times}(-) \rightarrow \Phi^{\mathrm{gp}}=\underline{\Phi}^{\mathrm{gp}}$ (cf. Proposition 4.4(iii)) and $\overline{\mathcal{C}}$ is of model type. Then there exists an equivalence of categories

$$
\mathcal{C} \stackrel{\sim}{\rightarrow} \underline{\mathcal{C}}
$$

that is 1-compatible with the functors $\mathcal{C} \rightarrow \mathbb{F}_{\Phi}, \underline{\mathcal{C}} \rightarrow \mathbb{F}_{\Phi}$.

Proof. Assertions (i) and (ii) follow via a routine verification (which, in the case of assertion (ii), is reminiscent of the verification that 'elementary Frobenioids are Frobenioids' in Proposition 1.5(i)); in light of assertion (ii), assertion (iii) follows formally from the definitions (cf. Definitions 3.1(i) and 4.5(iii)). Here, we observe that the objects $A=\left(A_{\mathcal{D}}, \alpha\right)$ such that $\alpha=0$ are Frobenius-trivial, and that these objects, together with the morphisms $\phi=\left(\operatorname{deg}_{\mathrm{Fr}}(\phi), \operatorname{Base}(\phi), \operatorname{Div}(\phi), u_{\phi}\right): A \rightarrow B$ such that $\operatorname{Div}(\phi)=0, u_{\phi}=1$ (i.e., $u_{\phi}$ is the identity element of $\mathbb{B}(A)$ ), determine a base-Frobenius pair of $\underline{\mathcal{C}}$. 
Finally, we consider assertion (iv). We may assume without loss of generality that $\mathcal{C}$, hence also $\mathcal{C}^{\mathrm{Fr}-\mathrm{tr}}$, is a skeleton. Let $(\mathcal{P}, \mathcal{F})$ be a base-Frobenius pair of $\mathcal{C}$ (cf. our assumption that $\mathcal{C}$ is of model type). Thus, $\mathcal{P}$ may be regarded as a subcategory of $\mathcal{C}^{\text {Fr-tr }}$. If $C \in \mathrm{Ob}(\mathcal{C})$, then let us refer to a(n) (ordered) pair of pre-steps in $\mathcal{C}$

$$
(B \rightarrow A, A \rightarrow C)
$$

such that $A \in \mathrm{Ob}(\mathcal{P})$ as an $\mathcal{F} \mathcal{P}$-path for $C$. Write

for the category $\mathcal{C}^{\prime}$ whose objects are objects of $\mathcal{C}$ equipped with an $\mathcal{F} \mathcal{P}$-path and whose morphisms are the morphisms between the objects regarded as objects of $\mathcal{C}$. Thus, we have a natural functor $\mathcal{C}^{\prime} \rightarrow \mathcal{C}$ (obtained by forgetting the $\mathcal{F} \mathcal{P}$-paths), which is manifestly an equivalence of categories. Thus, it suffices to construct an equivalence of categories $\mathcal{C}^{\prime} \stackrel{\sim}{\rightarrow} \underline{\mathcal{C}}$ that is compatible with the functors $\mathcal{C}^{\prime} \rightarrow \mathcal{C} \rightarrow \mathbb{F}_{\Phi}, \underline{\mathcal{C}} \rightarrow \mathbb{F}_{\Phi}$.

Next, observe that we may apply Remark 2.7.2 to $\mathcal{C}^{\text {Fr-tr }}$ (which is of base-trivial type, by Theorem 5.1(iii)) to conclude that every morphism $\phi$ of $\mathcal{C}^{\mathrm{Fr}-\mathrm{tr}}$ admits a unique factorization

$$
\phi=\phi_{\mathcal{P}} \circ \phi_{\mathcal{O}} \times \phi_{\mathcal{F}}
$$

in $\mathcal{C}^{\text {Fr-tr }}$, where $\phi_{\mathcal{P}}$ is $\mathcal{P}$-distinguished, $\phi_{\mathcal{O}^{\times}}$is a base-identity automorphism and $\phi_{\mathcal{F}}$ is $\mathcal{F}$ distinguished. Let us write

$$
\mathcal{E} \subseteq \mathcal{C}^{\text {birat }}
$$

for the full subcategory determined by the image of the objects in $\mathcal{P}$. Then observe that the category $\mathcal{E}$ is also a skeleton, that the Frobenioid $\mathcal{E} \stackrel{\sim}{\rightarrow} \mathcal{C}^{\text {birat }}$ is also of isotropic and basetrivial type (cf. Proposition 4.8(i) and Theorem 5.1(iii)) and that $(\mathcal{P}, \mathcal{F})$ determine a baseFrobenius pair of $\mathcal{E}$. Thus, we may apply Remark 2.7 .2 to $\mathcal{E}$ to conclude that every morphism $\psi$ of $\mathcal{E}$ admits a unique factorization

$$
\psi=\psi_{\mathcal{P}} \circ \psi_{\mathcal{O}} \circ \psi_{\mathcal{F}}
$$

in $\mathcal{E}$, where $\psi_{\mathcal{P}}$ is $\mathcal{P}$-distinguished, $\psi_{\mathcal{O}^{\times}}$is a base-identity automorphism and $\psi_{\mathcal{F}}$ is $\mathcal{F}$-distinguished.

Now observe that to every object $C \in \mathrm{Ob}(\mathcal{C})$ equipped with an $\mathcal{F P}$-path $\left(\zeta_{A}: B \rightarrow\right.$ $A, \zeta_{C}: B \rightarrow C$ ), we may associate an object

$$
\left(\operatorname{Base}(A), \Phi\left(\zeta_{A}\right)^{-1}\left(\operatorname{Div}\left(\zeta_{C}\right)-\operatorname{Div}\left(\zeta_{A}\right)\right) \in \Phi^{\mathrm{gp}}(A)\right)
$$

of $\underline{\mathcal{C}}$ (cf. Theorem 5.1(i)). If $C^{\prime} \in \mathrm{Ob}(\mathcal{C})$ is equipped with an $\mathcal{F P}$-path $\left(\zeta_{A^{\prime}}: B^{\prime} \rightarrow A^{\prime}, \zeta_{C^{\prime}}\right.$ : $B^{\prime} \rightarrow C^{\prime}$ ), then we may associate to any morphism $\phi: C \rightarrow C^{\prime}$ a morphism

$$
\begin{aligned}
& \left(\operatorname{deg}_{\mathrm{Fr}}(\phi), \quad \operatorname{Base}\left(\zeta_{A^{\prime}}\right) \circ \operatorname{Base}\left(\zeta_{C^{\prime}}\right)^{-1} \circ \operatorname{Base}(\phi) \circ \operatorname{Base}\left(\zeta_{C}\right) \circ \operatorname{Base}\left(\zeta_{A}\right)^{-1}: A \rightarrow A^{\prime},\right. \\
& \quad\left(\Phi\left(\zeta_{A}\right)^{-1} \circ \Phi\left(\zeta_{C}\right)\right)(\operatorname{Div}(\phi)) \in \Phi(A), \\
& \left.\quad\left\{\zeta_{A^{\prime}}^{\text {birat }} \circ\left(\zeta_{C^{\prime}}^{\text {birat }}\right)^{-1} \circ \phi^{\text {birat }} \circ \zeta_{C}^{\text {birat }} \circ\left(\zeta_{A}^{\text {birat }}\right)^{-1}\right\}_{\mathcal{O}^{\times}} \in \mathcal{O}^{\times}\left(A^{\text {birat }}\right)\right)
\end{aligned}
$$

(where the superscript 'birat' denotes the images of the respective objects and morphisms of $\mathcal{C}$ in $\mathcal{C}^{\text {birat }}$ ) of $\mathcal{C}$. Now in light of the fact that $\mathcal{C}$ is of model - hence, in particular, birationally 
Frobenius-normalized - type, it is a routine exercise to verify that these assignments determine a functor

$$
\mathcal{C}^{\prime} \rightarrow \underline{\mathcal{C}}
$$

that is compatible with the functors $\mathcal{C}^{\prime} \rightarrow \mathcal{C} \rightarrow \mathbb{F}_{\Phi}, \underline{\mathcal{C}} \rightarrow \mathbb{F}_{\Phi}$. Indeed, this is immediate for the first three entries of the data that define a morphism of $\underline{\mathcal{C}}$; for the final entry, it follows from the existence of the unique factorizations of morphisms of $\mathcal{E}$ discussed above. Note that these factorizations also imply that this functor $\mathcal{C}^{\prime} \rightarrow \underline{\mathcal{C}}$ is faithful. Moreover, this functor $\mathcal{C}^{\prime} \rightarrow \underline{\mathcal{C}}$ is manifestly essentially surjective (cf. Theorem 5.1(i)) and full (cf. Theorem 5.1(ii)), hence an equivalence of categories, as desired. This completes the proof of assertion (iv).

Remark 5.2.1. It follows formally from Theorem 5.2(ii) and (iv), that the Frobenioid ' $C$ ' of Example 4.6 constitutes an example of a Frobenioid of isotropic, standard, and (strictly) rational type, which is not of group-like or model type.

PROPOSITION 5.3. (Realifications of Frobenioids) Suppose that $\Phi$ is perf-factorial. Then we shall refer to the model Frobenioid ( $c f$. Theorem 5.2(ii)) associated to the divisor monoid

$$
\Phi^{\mathrm{rlf}}
$$

(i.e., the 'realification' of Definition 2.4(i)) as the realification

$$
\mathcal{C}^{\text {rlf }}
$$

of the Frobenioid $\mathcal{C}$ and the rational function monoid $\mathbb{R} \cdot \Phi^{\text {birat }} \subseteq\left(\Phi^{\text {rlf }}\right)^{\text {gp }}$ (i.e., for $A_{\mathcal{D}} \in$ $\mathrm{Ob}(\mathcal{D}),\left(\mathbb{R} \cdot \Phi^{\text {birat }}\right)\left(A_{\mathcal{D}}\right)$ is the $\mathbb{R}$-vector subspace of $\left(\Phi^{\mathrm{rlf}}\right)^{\mathrm{gp}}\left(A_{\mathcal{D}}\right)$ generated by $\left.\Phi^{\text {birat }}\left(A_{\mathcal{D}}\right)\right)$. Moreover, the Frobenioid $\mathcal{C}^{\mathrm{un}-\mathrm{tr}}$ (respectively, $\left.\left(\mathcal{C}^{\mathrm{un}-\mathrm{tr}}\right)^{\mathrm{pf}}\right)$ is of model type and may be obtained as the model Frobenioid associated to the divisor monoid $\Phi$ (respectively, $\Phi^{\mathrm{pf}}$ ) and the rational function monoid $\Phi^{\text {birat }}$ (respectively, $\mathbb{Q} \cdot \Phi^{\text {birat }}=\Phi^{\text {birat }} \otimes_{\mathbb{Z}} \mathbb{Q}=\left(\Phi^{\text {birat }}\right)^{\text {pf }}$ ). In particular, if $\mathcal{C}$ is of Frobenius-isotropic type, then there is a natural 1-commutative diagram of functors

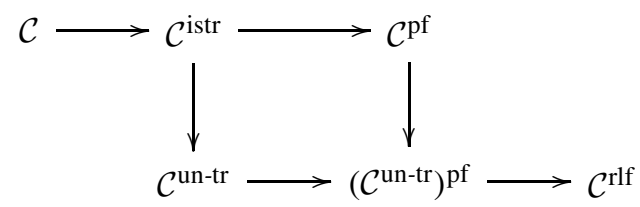

(where the functor $\mathcal{C} \rightarrow \mathcal{C}^{\text {istr }}$ is the isotropification functor of Proposition 1.9(v); the remaining functors are the functors that arise naturally from the construction of the 'unittrivialization', 'perfection' and 'realification').

Proof. Since Frobenioids of unit-trivial type are always of model type (cf. Theorem 5.1(iv)), the various assertions in the statement of Proposition 5.3 follow immediately from the definitions and Theorem 5.2(ii) and (iv).

COROLlary 5.4. (Category-theoreticity of the realification) For $i=1$, 2, let $\Phi_{i}$ be a perffactorial divisorial monoid on a connected, totally epimorphic category $\mathcal{D}_{i}$ which is Div-slim (with respect to $\Phi_{i}$ ), let $\mathcal{C}_{i} \rightarrow \mathbb{F}_{\Phi_{i}}$ be a Frobenioid of rationally standard type and let

$$
\Psi: \mathcal{C}_{1} \stackrel{\sim}{\rightarrow} \mathcal{C}_{2}
$$


be an equivalence of categories. If $\mathcal{C}_{1}, \mathcal{C}_{2}$ are of group-like type, then we also assume that both $\Psi$ and some quasi-inverse to $\Psi$ preserve base-isomorphisms. Then there exists a 1unique functor $\Psi^{\mathrm{rlf}}: \mathcal{C}_{1}^{\mathrm{rlf}} \rightarrow \mathcal{C}_{2}^{\mathrm{rlf}}$ that fits into a 1-commutative diagram

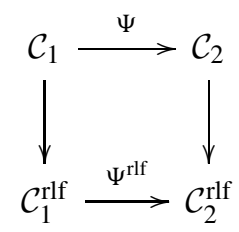

(where the vertical arrows are the natural functors of Proposition 5.3 and the horizontal arrows are equivalences of categories). Moreover, each of the composite functors of this diagram is rigid. Finally, the formation of $\Psi^{\mathrm{rlf}}$ from $\Psi$ is 1-compatible with the 1-commutative diagram of Proposition 5.3 (involving perfections, unit-trivializations, etc.).

Proof. In light of the definition of the realification (cf. Proposition 5.3), Corollary 5.4 follows immediately from Corollaries 4.10 and 4.11(iii) and (iv). (Here, we note that the rigidity assertion of Corollary 5.4 follows by a similar argument applied to prove the rigidity assertion in Corollary 4.11(i) and (iv).)

Before continuing, we note the following (portions of which were in fact applied in the proofs of Theorems 4.2 and 4.9).

Proposition 5.5. (Perfection, unit-trivialization and realification of types) Suppose that $\mathcal{C}$ is of Frobenius-isotropic and Frobenius-normalized type. Then we have the following.

(i) If $A \in \mathrm{Ob}\left(\mathcal{C}^{\text {istr }}\right)$ maps to an object $A^{\mathrm{pf}} \in \mathrm{Ob}\left(\mathcal{C}^{\mathrm{pf}}\right)$, then the natural functor $\mathcal{C} \rightarrow \mathcal{C}^{\mathrm{pf}}$ determines a natural isomorphism $\mathcal{O}^{\triangleright}(A)^{\mathrm{pf}} \stackrel{\sim}{\rightarrow} \mathcal{O}^{\triangleright}\left(A^{\mathrm{pf}}\right)$.

(ii) There is a natural equivalence of categories (compatible with the functors to the respective elementary Frobenioids) between $\left(\mathcal{C}^{\mathrm{pf}}\right)^{\mathrm{un}-\mathrm{tr}}$ and $\left(\mathcal{C}^{\mathrm{un}-\mathrm{tr}}\right)^{\mathrm{pf}}$ and between $\left(\mathcal{C}^{\text {pf }}\right)^{\text {birat }}$ and $\left(\mathcal{C}^{\text {birat }}\right)^{\mathrm{pf}}$.

(iii) If $\mathcal{C}$ is of standard (respectively, rationally standard; model) type, then so is $\mathcal{C}^{\mathrm{pf}}$. Moreover, $\mathcal{C}^{\mathrm{un}-\mathrm{tr}}, \mathcal{C}^{\mathrm{rlf}}$ are always of model type. Finally, suppose further that $\mathcal{C}$ is not of group-like type. Then if $\mathcal{C}$ is of standard (respectively, rationally standard) type, then so are $\mathcal{C}^{\text {un-tr }}, \mathcal{C}^{\text {rlf }}$.

(iv) If $\mathcal{C}$ is the model Frobenioid associated to data $\Phi, \mathbb{B}, \operatorname{Div}_{\mathbb{B}}: \mathbb{B} \rightarrow \Phi^{\mathrm{gp}}$ (cf. Theorem 5.2(ii)), then there is a natural equivalence of categories (compatible with the functors to the respective elementary Frobenioids) between $\mathcal{C}^{\mathrm{pf}}$ (respectively, $\left.\mathcal{C}^{\mathrm{un}-\mathrm{tr}} ; \mathcal{C}^{\mathrm{rlf}}\right)$ and the model Frobenioid associated to the data $\Phi^{\mathrm{pf}}, \mathbb{B}^{\mathrm{pf}}, \mathbb{B}^{\mathrm{pf}} \rightarrow\left(\Phi^{\mathrm{gp}}\right)^{\mathrm{pf}}$ (respectively, $\left.\Phi, \Phi^{\text {birat }}, \Phi^{\text {birat }} \hookrightarrow \Phi^{\mathrm{gp}} ; \Phi^{\mathrm{rlf}}, \mathbb{R} \cdot \Phi^{\text {birat }}, \mathbb{R} \cdot \Phi^{\text {birat }} \hookrightarrow\left(\Phi^{\mathrm{rlf}}\right)^{\mathrm{gp}}\right)$.

Proof. Assertion (i) follows immediately for Frobenius-trivial A by considering base-identity endomorphisms of Frobenius type of $A$ and applying the hypothesis that $\mathcal{C}$ is of Frobeniusnormalized type; the case of arbitrary $A$ then follows by considering 'pairs of pre-steps' as in Theorem 5.1(i) (cf. also Definition 1.3(iii)(c)). Next, we consider assertion (ii). One checks immediately that (in light of our hypothesis that $\mathcal{C}$ is of Frobenius-isotropic type) we may assume without loss of generality that $\mathcal{C}$ is of isotropic type. Then it follows immediately from the definition of the perfection (cf. Definition 3.1(iii)) that it suffices 
to obtain natural bijections between the respective sets of morphisms between the images of two given objects of $\mathcal{C}$ in $\left(\mathcal{C}^{\text {pf }}\right)^{\text {un-tr }},\left(\mathcal{C}^{\text {un-tr }}\right)^{\text {pf }}$ (respectively, $\left.\left.\left(\mathcal{C}^{\text {pf }}\right)^{\text {birat }}\right),\left(\mathcal{C}^{\text {birat }}\right)^{\text {pf }}\right)$. But this follows immediately from the definitions, together with Proposition 3.2(ii) applied to 'pre-steps' and 'units' (i.e., base-identity automorphisms). Next, we consider assertion (iii). First, we observe that $\mathcal{C}^{\text {un-tr }}, \mathcal{C}^{\text {rlf }}$ are of model type (cf. Theorem 5.1(iv), Proposition 5.3 and Theorem 5.2(ii)), hence of isotropic and birationally Frobenius-normalized type (cf. Definitions 2.7(iii) and 4.5(i)). Next, let us observe that by assertion (ii), we have natural equivalences $\left(\left(\mathcal{C}^{\text {un-tr }}\right)^{\text {birat }}\right)^{\mathrm{pf}} \stackrel{\sim}{\rightarrow}\left(\left(\mathcal{C}^{\text {pf }}\right)^{\text {un-tr }}\right)^{\text {birat }},\left(\mathcal{C}^{\text {birat }}\right)^{\mathrm{pf}} \stackrel{\sim}{\rightarrow}\left(\mathcal{C}^{\text {pf }}\right)^{\text {birat }}$; moreover, since $\mathcal{C}^{\text {un-tr }}$ is of birationally Frobenius-normalized type, it follows that $\left(\mathcal{C}^{\text {un-tr }}\right)^{\text {birat }}$ is of Frobeniusnormalized type, so assertion (i) may be applied to $\left(\mathcal{C}^{\text {un-tr }}\right)^{\text {birat }}$. In light of these observations, assertion (iii) for $\mathcal{C}^{\text {pf }}$ follows immediately from the definitions (cf. also Proposition 3.2(ii), (iii)) by observing that $\mathcal{C}^{\text {pf }}$ is of isotropic type, and that by assertion (i), if $\mathcal{C}^{*}$ is $\mathcal{C}$ or $\left(\mathcal{C}^{\text {un-tr }}\right)^{\text {birat }}$ (or $\mathcal{C}^{\text {birat }}$, when $\mathcal{C}$ is of birationally Frobenius-normalized type) and $A \in \operatorname{Ob}\left(\left(\mathcal{C}^{*}\right)^{\text {istr }}\right)$, then $\mathcal{O}^{\triangleright}(-)$ of the image of $A$ in $\left(\mathcal{C}^{*}\right)^{\mathrm{pf}}$ is the perfection of $\mathcal{O}^{\triangleright}(A)$. Now suppose that $\mathcal{C}$, hence also $\mathcal{C}^{\text {un-tr }}, \mathcal{C}^{\text {rlf }}$, are not of group-like type. Since $\left(\mathcal{C}^{\text {un-tr }}\right)^{\text {birat }}$ admits a Frobenius-compact object, the same is true for $\left(\mathcal{C}^{\text {rlf }}\right)^{\text {birat }}$. Also, we observe that the pull-back morphisms of $\mathcal{C}^{\text {un-tr }}$, $\mathcal{C}^{\text {rlf }}$ are precisely the linear isometries (cf. Proposition 1.4(ii)). In light of these observations, it follows immediately from the definitions that if $\mathcal{C}$ is of standard (respectively, rationally standard) type, then so are $\mathcal{C}^{\text {un-tr }}, \mathcal{C}^{\text {rlf }}$. Finally, assertion (iv) is immediate from the definitions (cf. also assertions (i) and (ii) and Proposition 5.3).

Finally, we conclude the theory of this section by discussing a certain issue which is closely related to the issue of being of model type. Namely, instead of working at the level of the entire category $\mathcal{C}$, or $\mathcal{C}^{\text {Fr-tr }}$, we consider the issue of being 'of model type' at the level of a single Frobenius-trivial object.

Proposition 5.6. (Base-sections of Frobenius-trivial objects) Suppose that $\mathcal{C}$ is of model (hence, in particular, isotropic - cf. Definition 2.7(iii)) and unit-profinite type. Let $(\mathcal{P}, \mathcal{F})$ be a base-Frobenius pair of $\mathcal{C}$, let $A \in \operatorname{Ob}(\mathcal{P})$ be a Frobenius-trivial object and let $A_{\mathcal{D}} \stackrel{\text { def }}{=} \operatorname{Base}(A)$. Then the pair

$$
\left(\sigma: \operatorname{Aut}_{\mathcal{D}}\left(A_{\mathcal{D}}\right) \hookrightarrow \operatorname{Aut}_{\mathcal{C}}(A), \quad \phi: \mathbb{N}_{\geq 1} \rightarrow \operatorname{End}_{\mathcal{C}}(A)\right)
$$

(where $\sigma$ is a group homomorphism whose composite with the natural surjection $\operatorname{Aut}_{\mathcal{C}}(A) \rightarrow$ $\operatorname{Aut}_{\mathcal{D}}\left(A_{\mathcal{D}}\right)$ (cf. Theorem 5.1(iii)) is the identity and $\phi$ is a homomorphism of monoids) determined by 'restricting' $\mathcal{P}, \mathcal{F}$ to $A$, in fact, depends only on the data $(\mathcal{C}, A)$ and, in particular, is independent of the data $(\mathcal{F}, \mathcal{P})$ - up to conjugation (as a pair!) by an element of $\mathcal{O}^{\times}(A)$. We shall refer to such a pair $(\sigma, \phi)$ as a base-Frobenius pair of $A$; when $\mathcal{F}$ is regarded as being known only up to composition with automorphisms of the monoid $\mathbb{N}_{\geq 1}$, we shall refer to such a pair as a quasi-base-Frobenius pair of A.

Proof. Let

$$
\left(\sigma^{\prime}: \operatorname{Aut}_{\mathcal{D}}\left(A_{\mathcal{D}}\right) \hookrightarrow \operatorname{Aut}_{\mathcal{C}}(A), \quad \phi^{\prime}: \mathbb{N}_{\geq 1} \rightarrow \operatorname{End}_{\mathcal{C}}(A)\right)
$$

be another such pair that arises from a base-Frobenius pair $\left(\mathcal{P}^{\prime}, \mathcal{F}^{\prime}\right)$ of $\mathcal{C}$. Write $\mathbb{E} \subseteq \operatorname{End}_{\mathcal{C}}(A)$ for the submonoid of base-isomorphisms; $\phi_{n} \stackrel{\text { def }}{=} \phi(n) \in \mathbb{E}, \phi_{n}^{\prime} \stackrel{\text { def }}{=} \phi^{\prime}(n) \in \mathbb{E}$, for $n \in \mathbb{N}_{\geq 1}$. Then I claim that it suffices to show the existence of a $u \in \mathcal{O}^{\times}(A) \subseteq \mathbb{E}$ such that

$$
u \cdot \phi_{p} \cdot u^{-1}=\phi_{p}^{\prime}
$$


for all $p \in \mathfrak{P r i m e s}$. Indeed, if, for $\alpha \in \operatorname{Aut}_{\mathcal{D}}\left(A_{\mathcal{D}}\right)$, we write $\sigma_{\alpha} \stackrel{\text { def }}{=} \sigma(\alpha), \sigma_{\alpha}^{\prime} \stackrel{\text { def }}{=} \sigma^{\prime}(\alpha)-$ so $\sigma_{\alpha}^{\prime}=v_{\alpha} \cdot u \cdot \sigma_{\alpha} \cdot u^{-1}$ for some $v_{\alpha} \in \mathcal{O}^{\times}(A) \subseteq \mathbb{E}$ - then it follows from the functoriality of $\mathcal{F}, \mathcal{F}^{\prime}$ that, for $p \in \mathfrak{P r i m e s}$,

$$
\sigma_{\alpha} \cdot \phi_{p}=\phi_{p} \cdot \sigma_{\alpha}, \quad \sigma_{\alpha}^{\prime} \cdot \phi_{p}^{\prime}=\phi_{p}^{\prime} \cdot \sigma_{\alpha}^{\prime},
$$

hence (since $\mathcal{C}$, being of model type, is also of (birationally) Frobenius-normalized type - cf. Definition 4.5(i)) that

$$
\begin{aligned}
u \cdot v_{\alpha} \cdot \phi_{p} \cdot \sigma_{\alpha} \cdot u^{-1} & =v_{\alpha} \cdot u \cdot \sigma_{\alpha} \cdot \phi_{p} \cdot u^{-1}=v_{\alpha} \cdot\left(u \cdot \sigma_{\alpha} \cdot \phi_{p} \cdot u^{-1}\right) \\
& =v_{\alpha} \cdot\left(u \cdot \sigma_{\alpha} \cdot u^{-1}\right) \cdot\left(u \cdot \phi_{p} \cdot u^{-1}\right)=\sigma_{\alpha}^{\prime} \cdot \phi_{p}^{\prime}=\phi_{p}^{\prime} \cdot \sigma_{\alpha}^{\prime} \\
& =\left(u \cdot \phi_{p} \cdot u^{-1}\right) \cdot v_{\alpha} \cdot\left(u \cdot \sigma_{\alpha} \cdot u^{-1}\right) \\
& =\left(u \cdot v_{\alpha}^{p} \cdot \phi_{p} \cdot u^{-1}\right) \cdot\left(u \cdot \sigma_{\alpha} \cdot u^{-1}\right) \\
& =u \cdot v_{\alpha}^{p} \cdot \phi_{p} \cdot \sigma_{\alpha} \cdot u^{-1}
\end{aligned}
$$

which (by the total epimorphicity of $\mathcal{C}$ ) implies that $v_{\alpha}=v_{\alpha}^{p}$, for all $p \in \mathfrak{P r i m e s}$. Thus, by taking $p=2$, we obtain that $v_{\alpha}=1$. Since $\phi, \phi^{\prime}$ are homomorphisms, and $\mathbb{N}_{\geq 1}$ is generated by $\mathfrak{P r i m e s}$, this completes the proof of the claim.

To verify the existence of a $u \in \mathcal{O}^{\times}(A)$ as in the above claim, let us first observe that if $M \subseteq \mathcal{O}^{\times}(A) \subseteq \mathbb{E}$ is any subgroup such that for any $m \in M, f \in \mathbb{E}$, there exists an $m^{\prime} \in M$ such that $f \cdot m=m^{\prime} \cdot f$, then there is a natural monoid structure on the set of cosets $\mathbb{E}_{M} \stackrel{\text { def }}{=} M \backslash \mathbb{E}=\{M \cdot f\}_{f \in \mathbb{E}}$, together with a natural surjection of monoids $\mathbb{E} \rightarrow \mathbb{E}_{M}$. For $p \in \mathfrak{P r i m e s}$, let us write

$$
M_{p} \subseteq \mathcal{O}^{\times}(A)
$$

for the closed subgroup topologically generated by the pro-l portions $\left(\mathcal{O}^{\times}(A)\right)[l]$ (cf. Definition 2.8(ii)) of $\mathcal{O}^{\times}(A)$, as $l$ ranges over the primes $\neq p$. Note that since the Frobenioid $\mathcal{C}^{\text {Fr-tr }}$ is of Aut-ample type (cf. Theorem 5.1(iii)), it follows that any $f \in \mathbb{E}$ admits a factorization $f=f_{0} \cdot f_{1}$, where $f_{0}$ is an automorphism, and $f_{1}$ is a base-identity endomorphism. Thus, (by applying, again, the fact that $\mathcal{C}$, being of model type, is also of (birationally) Frobenius-normalized type - cf. Definition 4.5(i)) it follows that 'for any $m \in M_{p}$, there exists an $m^{\prime} \in M_{p}$ such that $f \cdot m=m^{\prime} \cdot f^{\prime}$. In particular, it makes sense to speak of the monoid $\mathbb{E}_{M_{p}}$. Let us use the symbol $\stackrel{p}{\approx}$, to denote the equality of the images in $\mathbb{E}_{p}$ of elements of $\mathbb{E}$. Now since we have a natural isomorphism

$$
\prod_{p \in \mathfrak{P r i m e s}} \mathcal{O}^{\times}(A)[p] \stackrel{\sim}{\rightarrow} \mathcal{O}^{\times}(A)
$$

(cf. Definition 2.8(ii)), it thus follows that to prove the existence of a $u \in \mathcal{O}^{\times}(A)$ as desired, it suffices to show, for each $p \in \mathfrak{P r i m e s}$, the existence of a $u_{p} \in \mathcal{O}^{\times}(A)[p]$ such that $u_{p} \cdot \phi_{l} \cdot u_{p}^{-1} \stackrel{p}{\approx} \phi_{l}^{\prime}$, for all $l \in \mathfrak{P r i m e s}$ (i.e., we then take $u$ to be the 'infinite product' of the $u_{p}$ ).

Now observe that for each $l \in \mathfrak{P r i m e s}, \phi_{l}^{\prime} \stackrel{p}{\approx} v_{l} \cdot \phi_{l}$, for some $v_{l} \in \mathcal{O}^{\times}(A)[p]$. Since, for $w \in \mathcal{O}^{\times}(A)[p]$, we have, for $l \in \mathfrak{P r i m e s}, w \cdot \phi_{l} \cdot w^{-1} \stackrel{p}{\approx} w^{1-l} \cdot \phi_{l}$ (where we recall again that $\mathcal{C}$, being of model type, is also of (birationally) Frobenius-normalized type - 
cf. Definition 4.5(i)), and $\mathcal{O}^{\times}(A)[p]$ is a (topologically finitely generated) pro-p group, it follows that there exists a $u_{p} \in \mathcal{O}^{\times}(A)[p]$ such that $u_{p} \cdot \phi_{p} \cdot u_{p}^{-1} \stackrel{p}{\approx} \phi_{p}^{\prime}$, as well as a $w_{l} \in \mathcal{O}^{\times}(A)[p]$ such that $w_{l} \cdot u_{p} \cdot \phi_{l} \cdot u_{p}^{-1} \stackrel{p}{\approx} \phi_{l}^{\prime}$, for each $l \in \mathfrak{P r i m e s}$. On the other hand, since $\phi, \phi^{\prime}$ are homomorphisms, it follows that

$$
\phi_{l_{1}} \cdot \phi_{l_{2}} \stackrel{p}{\approx} \phi_{l_{2}} \cdot \phi_{l_{1}}, \quad \phi_{l_{1}}^{\prime} \cdot \phi_{l_{2}}^{\prime} \stackrel{p}{\approx} \phi_{l_{2}}^{\prime} \cdot \phi_{l_{1}}^{\prime}
$$

(for $l_{1}, l_{2} \in \mathfrak{P r i m e s}$ ). Thus, for $l \in \mathfrak{P r i m e s}$, we have

$$
\begin{aligned}
W_{l} \cdot u_{p} \cdot \phi_{p} \cdot \phi_{l} \cdot u_{p}^{-1} & \stackrel{p}{\approx} w_{l} \cdot u_{p} \cdot \phi_{l} \cdot \phi_{p} \cdot u_{p}^{-1} \stackrel{p}{\approx} w_{l} \cdot u_{p} \cdot \phi_{l} \cdot u_{p}^{-1} \cdot u_{p} \cdot \phi_{p} \cdot u_{p}^{-1} \\
& \stackrel{p}{\approx} \phi_{l}^{\prime} \cdot \phi_{p}^{\prime} \stackrel{p}{\approx} \phi_{p}^{\prime} \cdot \phi_{l}^{\prime} \stackrel{p}{\approx} u_{p} \cdot \phi_{p} \cdot u_{p}^{-1} \cdot w_{l} \cdot u_{p} \cdot \phi_{l} \cdot u_{p}^{-1} \\
& \stackrel{p}{\approx} u_{p} \cdot w_{l}^{p} \cdot \phi_{p} \cdot u_{p}^{-1} \cdot u_{p} \cdot \phi_{l} \cdot u_{p}^{-1} \stackrel{p}{\approx} w_{l}^{p} \cdot u_{p} \cdot \phi_{p} \cdot \phi_{l} \cdot u_{p}^{-1}
\end{aligned}
$$

which (by the total epimorphicity of $\mathcal{C}$ ) implies that $w_{l} \stackrel{p}{\approx} w_{l}^{p}$ (for all $l \in \mathfrak{P r i m e s}$ ). Since $\mathcal{O}^{\times}(A)[p]$ is a (topologically finitely generated) pro-p group, we thus conclude that $w_{l} \stackrel{p}{\approx} 1$. This completes the proof of the existence of a $u \in \mathcal{O}^{\times}(A)$ as desired, and hence of Proposition 5.6.

Remark 5.6.1. The notion of a 'base-section of a Frobenius-trivial object' (i.e., in the notation of Proposition 5.6, a section ' $\sigma$ ') is intended to be an abstract category-theoretic translation of the notion of a 'tautological section of a trivial line bundle' (cf. Remark 2.7.1 and the Frobenioids of Examples 6.1 and 6.3 below).

COROLlary 5.7. (Category-theoreticity of base-sections) For $i=1,2$, let $\Phi_{i}$ be a perffactorial divisorial monoid on a connected, totally epimorphic category $\mathcal{D}_{i}$ which is Div-slim (with respect to $\Phi_{i}$ ), let $\mathcal{C}_{i} \rightarrow \mathbb{F}_{\Phi_{i}}$ be a Frobenioid of standard type and let

$$
\Psi: \mathcal{C}_{1} \stackrel{\sim}{\rightarrow} \mathcal{C}_{2}
$$

be an equivalence of categories. If $\mathcal{C}_{1}, \mathcal{C}_{2}$ are of group-like type, then we also assume that both $\Psi$ and some quasi-inverse to $\Psi$ preserve base-isomorphisms. Then we have the following.

(i) $\Psi$ maps base-sections (respectively, quasi-base-Frobenius pairs) of $\mathcal{C}_{1}$ to base-sections (respectively, quasi-base-Frobenius pairs) of $\mathcal{C}_{2}$. In particular, $\mathcal{C}_{1}$ is of model type if and only if $\mathcal{C}_{2}$ is.

(ii) $\mathcal{C}_{1}$ is of unit-profinite type if and only if $\mathcal{C}_{2}$ is.

(iii) Suppose that $\mathcal{C}_{1}, \mathcal{C}_{2}$ are of model and unit-profinite type. Then $\Psi$ maps every quasi-base-Frobenius pair of a Frobenius-trivial object $A_{1} \in \mathrm{Ob}\left(\mathcal{C}_{1}\right)$ to a quasi-baseFrobenius pair of a Frobenius-trivial object $A_{2} \in \mathrm{Ob}\left(\mathcal{C}_{2}\right)$.

(iv) Suppose, moreover, when $\mathcal{C}_{1}, \mathcal{C}_{2}$ are of group-like type, that both $\Psi$ and some quasiinverse to $\Psi$ preserve Frobenius degrees. Then the prefix 'quasi-' may be removed from the statements of (i) and (iii).

Proof. Indeed, sorting through the definitions, to verify assertions (i)-(iv) it suffices to show that $\Psi$ preserves isotropic objects, prime-Frobenius morphisms, pull-back morphisms, birationalizations, the natural projection functor $\mathcal{C}_{i} \rightarrow \mathcal{D}_{i}$ (hence, in particular, the units 
' $\mathcal{O}^{\times}(-)$') and (in the case of the final portion of assertion (iv), when $\mathcal{C}_{1}, \mathcal{C}_{2}$ are not of grouplike type) Frobenius degrees. But this follows from Theorem 3.4(i), (iii), Corollaries 4.10 and 4.11(ii) (cf. also Remark 3.4.1). This completes the proof of Corollary 5.7.

\section{Some motivating examples}

In this section, we discuss some of the principal motivating examples from arithmetic geometry of the theory of Frobenioids. In particular, in the case of number fields, one of these examples provides an interesting 'category-theoretic interpretation' of some results of classical number theory, such as the Dirichlet unit theorem and Tchebotarev's density theorem, as well as a result in transcendence theory due to Lang (cf. Theorem 6.4(i), (iii) and (iv)).

Example 6.1. (A Frobenioid of geometric origin) Let $V$ be a proper normal (geometrically integral) variety over a field $k, K$ the function field of $V, \widetilde{K} / K$ a (possibly infinite) Galois extension, $G \stackrel{\text { def }}{=} \operatorname{Gal}(\widetilde{K} / K)$ and $\mathbb{D}_{K}$ a set of $\mathbb{Q}$-Cartier prime divisors on $V$. The connected objects of the Galois category $\mathcal{B}(G)$ (cf. Section 0 ) may be thought of as schemes $\operatorname{Spec}(L)$, where $L \subseteq \widetilde{K}$ is a finite (necessarily separable) extension of $K$. If we write $V[L]$ for the normalization of $V$ in $L$ (so $V[L]$ is also a proper normal variety), then let us write $\mathbb{D}_{L}$ for the set of prime divisors of $V[L]$ that map into (possibly subvarieties of codimension $\geq 1$ of) prime divisors of $\mathbb{D}_{K}$. If for every $\operatorname{Spec}(L) \in \operatorname{Ob}\left(\mathcal{B}(G)^{0}\right)$ (cf. Section 0 ), every prime divisor of $\mathbb{D}_{L}$ is $\mathbb{Q}$-Cartier, then we shall say that $\mathbb{D}_{K}$ is $\widetilde{K}$ - $\mathbb{Q}$-Cartier. In the following, we shall assume that $\mathbb{D}_{K}$ is $\widetilde{K}$ - $\mathbb{Q}$-Cartier. If $L \subseteq \widetilde{K}$ is a finite extension, then let us write

$$
\Phi(L) \subseteq \mathbb{Z}_{\geq 0}\left[\mathbb{D}_{L}\right] \subseteq \mathbb{Z}\left[\mathbb{D}_{L}\right]
$$

for the monoid of Cartier effective divisors $D$ on $V[L]$ with support in $\mathbb{D}_{L}$ (i.e., $D$ such that every prime divisor in the support of $D$ belongs to $\mathbb{D}_{L}$ ) and

$$
\mathbb{B}(L) \subseteq L^{\times}
$$

for the group of rational functions $f$ on $V[L]$ such that every prime divisor at which $f$ has a zero or a pole belongs to $\mathbb{D}_{L}$. Observe that $\Phi(L)^{\mathrm{gp}} \subseteq \mathbb{Z}\left[\mathbb{D}_{L}\right]$ may be identified with the group of Cartier divisors on $V[L]$ and that

$$
\Phi(L)^{\mathrm{pf}}=\mathbb{Q}_{\geq 0}\left[\mathbb{D}_{L}\right] \subseteq \mathbb{Q}\left[\mathbb{D}_{L}\right]=\left(\Phi(L)^{\mathrm{pf}}\right)^{\mathrm{gp}}
$$

(since $\mathbb{D}_{K}$ is $\widetilde{K}$ - $\mathbb{Q}$-Cartier); moreover, one verifies immediately that $\Phi(L)$ is perf-factorial, that there is a natural bijection Prime $(\Phi(L)) \stackrel{\sim}{\rightarrow} \mathbb{D}_{L}$ and that the supports of elements of $\Phi(L)$ are precisely the finite subsets of $\mathbb{D}_{L}$. By assigning to a rational function $f$ the divisor obtained by subtracting the divisor of poles of $f$ from the divisor of zeroes of $f$, we obtain a natural homomorphism

$$
\mathbb{B}(L) \rightarrow \Phi(L)^{\mathrm{gp}}
$$

which is functorial in $L$. In particular, the assignments $L \mapsto \Phi(L), L \mapsto \mathbb{B}(L)$ determine, respectively, a perf-factorial divisorial monoid $\Phi$ on $\mathcal{D} \stackrel{\text { def }}{=} \mathcal{B}(G)^{0}$ and a group-like monoid $\mathbb{B}$ on $\mathcal{D}$, equipped with a homomorphism (of monoids on $\mathcal{D}$ ) $\mathbb{B} \rightarrow \Phi^{\mathrm{gp}}$. Thus, by 
Theorem 5.2(ii) this data determines a (model) Frobenioid

$$
\mathcal{C}_{V, \tilde{K}, \mathbb{D}_{K}}
$$

of isotropic and birationally Frobenius-normalized type. Note that an object of $\mathcal{C}_{V, \widetilde{K}, \mathbb{D}_{K}}$ that projects to $\operatorname{Spec}(L) \in \operatorname{Ob}\left(\mathcal{B}(G)^{0}\right)$ may be thought of as a line bundle $\mathcal{L}$ on $V[L]$ that is representable by a Cartier divisor $D$ with support in $\mathbb{D}_{L}$. If $\mathcal{L}$ is such a line bundle on $V[L]$ and $\mathcal{M}$ is such a line bundle on $V[M]$ (where $M \subseteq \widetilde{K}$ is a finite extension of $K$ ), then one verifies immediately that a morphism $\mathcal{L} \rightarrow \mathcal{M}$ in $\mathcal{C}_{V, \widetilde{K}, \mathbb{D}_{K}}$ may be thought of as consisting of the following data: (a) a morphism $\operatorname{Spec}(L) \rightarrow \operatorname{Spec}(M)$ over $\operatorname{Spec}(K)$ (which thus induces a morphism $V[L] \rightarrow V[M]$ over $V$ ); (b) an element $d \in \mathbb{N}_{\geq 1}$; (c) a morphism of line bundles $\left.\mathcal{L}^{\otimes d} \rightarrow \mathcal{M}\right|_{V[L]}$ on $V[L]$ whose zero locus is a Cartier divisor supported in $\mathbb{D}_{L}$. Also, we observe that (since $V[L]$ is a proper normal variety) for $A \in \mathrm{Ob}\left(\mathcal{C}_{V, \widetilde{K}, \mathbb{D}_{K}}\right)$ that projects to $\operatorname{Spec}(L) \in \operatorname{Ob}\left(\mathcal{B}(G)^{0}\right)$, we have

$$
\mathcal{O}^{\times}(A)=\mathcal{O}^{\triangleright}(A)=k_{L}^{\times},
$$

where $k_{L}$ denotes the algebraic closure of $k$ in $L$ (so $k_{L}$ is a finite separable extension of $k$ ).

THEOREM 6.2. (Geometric Frobenioids) For $i=1$, 2, let $V_{i}$ be a proper normal (geometrically integral) variety over a field $k_{i}, K_{i}$ the function field of $V_{i}, \widetilde{K}_{i} / K_{i}$ a (possibly infinite) Galois extension, $G_{i} \stackrel{\text { def }}{=} \operatorname{Gal}\left(\widetilde{K}_{i} / K_{i}\right), \mathcal{D}_{i} \stackrel{\text { def }}{=} \mathcal{B}\left(G_{i}\right)^{0}$ and let $\mathbb{D}_{K_{i}} \neq \emptyset$ be a $\widetilde{K}_{i}-\mathbb{Q}$-Cartier set of prime divisors on $V_{i}$. For $\operatorname{Spec}\left(L_{i}\right) \in \operatorname{Ob}\left(\mathcal{D}_{i}\right)$, write $V_{i}\left[L_{i}\right]$ for the normalization of $V_{i}$ in $L_{i}$ write $\mathbb{D}_{L_{i}}$ for the set of prime divisors of $V_{i}\left[L_{i}\right]$ that map into (possibly subvarieties of codimension $\geq 1$ of) prime divisors of $\mathbb{D}_{K_{i}}$, write

$$
\Phi_{i}\left(L_{i}\right) \subseteq \mathbb{Z}_{\geq 0}\left[\mathbb{D}_{L_{i}}\right] \subseteq \mathbb{Z}\left[\mathbb{D}_{L_{i}}\right]
$$

for the monoid of Cartier effective divisors on $V_{i}\left[L_{i}\right]$ with support in $\mathbb{D}_{L_{i}}$, write

$$
\mathbb{B}_{i}\left(L_{i}\right) \subseteq L_{i}^{\times}
$$

for the group of rational functions on $V_{i}\left[L_{i}\right]$ whose zeroes and poles are supported on $\mathbb{D}_{L_{i}}$, write $\mathbb{B}_{i}\left(L_{i}\right) \rightarrow \Phi_{i}\left(L_{i}\right)^{\mathrm{gp}}$ for the natural map, and write

$$
\mathcal{C}_{i}
$$

for the associated model Frobenioid of Theorem 5.2(ii). Then we have the following.

(i) Let

$$
\psi: V_{2} \rightarrow V_{1}
$$

be a dominant morphism of schemes such that the following conditions are satisfied: (a) $\mathbb{D}_{K_{2}}$ is equal to the set of prime divisors of $V_{2}$ that map into a prime divisor of $\mathbb{D}_{K_{1}}$; (b) the resulting inclusion of function fields $K_{1} \hookrightarrow K_{2}$ satisfies the condition that the composite inclusion $K_{1} \hookrightarrow K_{2} \hookrightarrow \widetilde{K}_{2}$ factors through $\widetilde{K}_{1}$; (c) $K_{1}$ is separably closed in $K_{2}$. Then $\psi$ induces a functor

$$
\Psi: \mathcal{C}_{1} \rightarrow \mathcal{C}_{2}
$$

(well-defined up to isomorphism) that is compatible with Frobenius degrees, the functor $\mathcal{D}_{1} \rightarrow \mathcal{D}_{2}$ induced by the inclusion of fields $K_{1} \hookrightarrow K_{2}$ and the natural transformations $\left.\Phi_{1} \rightarrow \Phi_{2}\right|_{\mathcal{D}_{1}},\left.\mathbb{B}_{1} \rightarrow \mathbb{B}_{2}\right|_{\mathcal{D}_{1}}$ induced by pulling back divisors and rational functions, respectively, via $\psi$. 
(ii) Assume that the data labeled by the index ' 1 ' is equal to the data labeled by the index ' 2 ' (so in the following, we shall omit these indices). Also, let us suppose that $k$ is of positive characteristic $p$. Then the Frobenius morphism $\psi: V \rightarrow V$ satisfies the conditions of (i), hence determines a functor

$$
\Psi: \mathcal{C} \rightarrow \mathcal{C}
$$

which is isomorphic to the naive Frobenius functor (of degree $p$ on $\mathcal{C}$ ) of Proposition 2.1.

(iii) We maintain the assumption of (ii) concerning indices. Then the Frobenioid $\mathcal{C}$ is of isotropic, standard and birationally Frobenius-normalized type, but not of group-like type. If, moreover, for every finite extension $L \subseteq \widetilde{K}$ of $K$, and every $D \in \mathbb{D}_{L}$, it holds that $D$ lies in the support of the image in $\Phi(L)^{\mathrm{gp}}$ of an element of $\mathbb{B}(L)$, then $\mathcal{C}$ is of rationally standard type.

(iv) We maintain the assumption of (ii) concerning indices. Then $\mathcal{D}$ is Frobenius-slim. Let $Z \subseteq G$ be the subgroup of elements that commute with some open subgroup of $G$. Then $\mathcal{D}$ is slim if and only if $Z=\{1\}$ and $\mathcal{D}$ is Div-slim (relative to $\Phi$ ) if and only if, for every $1 \neq z \in Z$, there exists a finite Galois extension $L \subseteq \widetilde{K}$ of $K$ such that $z$ acts non-trivially on $\Phi(L)$.

Proof. First, we consider assertion (i). Note that by assumptions (b) and (c) (in the statement of assertion (i)), it follows that any finite extension $L_{1} \subseteq \widetilde{K}_{1}$ of $K_{1}$ determines a finite extension $L_{2} \stackrel{\text { def }}{=} L_{1} \cdot K_{2} \subseteq \widetilde{K}_{2}$ of $K_{2}$ such that $\left[L_{2}: K_{2}\right]=\left[L_{1}: K_{1}\right]$. Thus, $\psi$ determines a functor $\mathcal{D}_{1} \rightarrow \mathcal{D}_{2}$. Moreover, by assumption (a) (in the statement of assertion (i)), it follows that by pulling back (Cartier) divisors and rational functions via $\psi$, we obtain compatible natural transformations $\left.\Phi_{1} \rightarrow \Phi_{2}\right|_{\mathcal{D}_{1}},\left.\mathbb{B}_{1} \rightarrow \mathbb{B}_{2}\right|_{\mathcal{D}_{1}}$. Thus, it follows formally from the definition of the category underlying a model Frobenioid in Theorem 5.2(i), that we obtain a functor $\Psi: \mathcal{C}_{1} \rightarrow \mathcal{C}_{2}$ satisfying the properties stated in assertion (i). From this definition of the functor $\Psi$, it then follows immediately from the definition of the 'Frobenius morphism in characteristic $p$ ', together with the definition of the 'naive Frobenius functor' of Proposition 2.1 - i.e., in a word, that both functors are obtained by 'raising to the pth power' - that these two functors are isomorphic. This completes the proof of assertions (i) and (ii).

Next, we consider assertion (iii). The fact that $\mathcal{C}$ is of isotropic and birationally Frobenius-normalized type follows formally from Theorem 5.2(ii). The fact that $\mathcal{C}$ is not of group-like type is immediate from our assumption that $\mathbb{D}_{K} \neq \emptyset$ (and the definition of $\Phi$ ). It is immediate that every monomorphism of $\mathcal{D}$ is an isomorphism, hence that $\mathcal{D}$ is of FSMtype (hence also of FSMFF-type - cf. Section 0). If a $K$-linear automorphism $\alpha$ of a finite extension $L \subseteq \widetilde{K}$ of $K$ induces an automorphism of $\Phi(L)$ which preserves the primes of $L$, then it is immediate from the fact that $\alpha$ induces an automorphism of the scheme $V[L]$ that $\alpha$ maps every prime divisor $D \in \Phi(L)$ to $D$ (i.e., not to some $n \cdot D$, where $n \geq 2$ ); thus, we conclude that $\Phi$ is non-dilating, hence that $\mathcal{C}$ is of standard type. Now suppose that for every finite extension $L \subseteq \widetilde{K}$ of $K$ and every $D \in \mathbb{D}_{L}$, it holds that $D$ lies in the support of the image in $\Phi(L)^{\mathrm{gp}}$ of an element of $\mathbb{B}(L)$. Then it follows formally (cf. Definition 4.5(ii)) that $\mathcal{C}$ is of (strictly) rational type (since $\Phi$ has already been observed to be perf-factorial cf. Example 6.1). Thus, $\mathcal{C}$ satisfies condition (a) of Definition 4.5(iii). Now I claim that every object of $\left(\mathcal{C}^{\text {un-tr }}\right)^{\text {birat }}$ is Frobenius-compact. Indeed, if $\alpha$ is a $K$-linear automorphism of a finite 
extension $L \subseteq \widetilde{K}$ of $K$ that acts by multiplication by $\lambda \in \mathbb{Q}>0$ on $\Phi^{\text {birat }}(L)^{\mathrm{pf}}(\neq 0)$, then since $\alpha$ induces an automorphism of the variety $V[L]$, it follows that the $\operatorname{order} \in \mathbb{Q}_{>0}$ of the zero (or pole) of highest order of an element $f \in \Phi^{\text {birat }}(L)^{\mathrm{pf}}$ is preserved by $\alpha$, hence that $\lambda=1$. This completes the proof of the claim, and hence of the fact that $\mathcal{C}$ is of rationally standard type.

Finally, we consider assertion (iv). First, we observe that if $L \subseteq \widetilde{K}$ is a finite extension of $K$ that corresponds to an open subgroup $H \subseteq G$, then there is a natural isomorphism

$$
(Z \supseteq) Z_{G}(H) \stackrel{\sim}{\rightarrow} \operatorname{Aut}\left(\mathcal{D}_{\operatorname{Spec}(L)} \rightarrow \mathcal{D}\right)
$$

(cf. [Mzk7, Corollary 1.1.6]). Since $G$ is profinite, hence, in particular, residually finite, it follows formally that $Z, Z_{G}(H)$ are also residually finite, hence that $\mathcal{D}$ is Frobenius-slim, by Remark 3.1.2. Also, since $Z$ is the union of subgroups of $G$ of the form ' $Z_{G}(H)$ ', it follows formally that $\mathcal{D}$ is slim if and only if $Z=\{1\}$ and that $\mathcal{D}$ is Div-slim (relative to $\Phi$ ) if and only if, for every $1 \neq z \in Z$, there exists a finite Galois extension $L^{\prime} \subseteq \widetilde{K}$ of $K$ such that $z$ acts non-trivially on $\Phi\left(L^{\prime}\right)$. This completes the proof of assertion (iv).

Remark 6.2.1. Theorem 6.2(ii) constitutes the principal justification for the name 'Frobenius functor' that was applied to various functors in Section 2. From this point of view, the decomposition of the naive Frobenius functor of Proposition 2.1 into 'unit-linear' and 'unitwise' Frobenius functors (cf. the proof of Corollary 2.6) may be thought of as corresponding to the decomposition of the Frobenius morphism in positive characteristic algebraic geometry over a fixed base into the composite of a 'relative Frobenius morphism', which is linear over the fixed base, with the Frobenius morphism of the fixed base.

Example 6.3. (A Frobenioid of arithmetic origin) Let $F$ be a number field (cf. Section 0). Write $\mathbb{V}(F)$ for the set of valuations on $F$ (where we identify complex archimedean valuations with their complex conjugates) and write $\mathcal{O}_{F}$ for the ring of integers of $F$. If $v \in \mathbb{V}(F)$, then we shall write $F_{v}$ for the completion of $F$ at $v$, write $\mathcal{O}_{v}^{\times} \subseteq F_{v}^{\times}$for the group of units (i.e., elements of valuation 1 of $F_{v}^{\times}$), write $\mathcal{O}_{v}^{\triangleright} \subseteq F_{v}^{\times}$for the multiplicative monoid of elements of valuation $\leq 1$, write $\boldsymbol{\mu}(F) \subseteq \mathcal{O}_{F}^{\times}$for the group of roots of unity in $F$, write $\operatorname{ord}\left(F_{v}\right) \stackrel{\text { def }}{=} F_{v}^{\times} / \mathcal{O}_{v}^{\times}$and $\operatorname{ord}\left(\mathcal{O}_{v}^{\triangleright}\right) \stackrel{\text { def }}{=} \mathcal{O}_{v}^{\triangleright} / \mathcal{O}_{v}^{\times} \subseteq \operatorname{ord}\left(F_{v}\right)$. Thus, $\operatorname{ord}\left(F_{v}\right)=\operatorname{ord}\left(\mathcal{O}_{v}^{\triangleright}\right)^{\mathrm{gp}}$ and $\operatorname{ord}\left(F_{v}\right) \cong \mathbb{Z}, \operatorname{ord}\left(\mathcal{O}_{v}^{\triangleright}\right) \cong \mathbb{Z}_{\geq 0}$ if $v$ is non-archimedean and $\operatorname{ord}\left(F_{v}\right) \cong \mathbb{R}, \operatorname{ord}\left(\mathcal{O}_{v}^{\triangleright}\right) \cong \mathbb{R}_{\geq 0}$ if $v$ is archimedean. We shall refer to an element of the monoid

$$
\Phi(F) \stackrel{\text { def }}{=} \bigoplus_{v \in \mathbb{V}(F)} \operatorname{ord}\left(\mathcal{O}_{v}^{\triangleright}\right)
$$

as an effective arithmetic divisor on $F$ and to an element of the group

$$
\Phi(F)^{\mathrm{gp}}=\bigoplus_{v \in \mathbb{V}(F)} \operatorname{ord}\left(F_{v}\right)
$$

as an arithmetic divisor on $F$. Thus, there is a natural homomorphism of groups

$$
\mathbb{B}(F) \stackrel{\text { def }}{=} F^{\times} \rightarrow \Phi(F)^{\text {gp }}
$$

(given by mapping an element $f \in F^{\times}$to the images of $f$ in the various factors $F_{v}^{\times} / \mathcal{O}_{v}^{\times}=$ $\operatorname{ord}\left(F_{v}\right)$, all but a finite number of which are zero). Note, moreover, that $\Phi, \mathbb{B}$, as well as the 
homomorphism $\mathbb{B} \rightarrow \Phi^{\mathrm{gp}}$ are functorial in the number field $F$. Thus, if $\widetilde{F}$ is a (not necessarily finite) Galois extension of $F, G \stackrel{\text { def }}{=} \operatorname{Gal}(\widetilde{F} / F), \mathcal{D} \stackrel{\text { def }}{=} \mathcal{B}(G)^{0}$, then $\Phi, \mathbb{B}$ determine monoids on $\mathcal{D}$, and we have a natural homomorphism $\mathbb{B} \rightarrow \Phi^{\mathrm{gp}}$. Moreover, for each finite extension $L \subseteq \widetilde{F}$ of $F$, one verifies immediately that $\Phi(L) \neq 0$ is perf-factorial, that there is a natural bijection Prime $(\Phi(L)) \stackrel{\sim}{\rightarrow} \mathbb{V}(L)$, and that the supports of elements of $\Phi(L)$ are precisely the finite subsets of $\mathbb{V}(L)$. Thus, by Theorem 5.2(ii), this data determines a (model) Frobenioid

$$
\mathcal{C}_{\widetilde{F} / F}
$$

of isotropic and birationally Frobenius-normalized type. Note that an object of $\mathcal{C}_{\widetilde{F} / F}$ that projects to $\operatorname{Spec}(L) \in \operatorname{Ob}\left(\mathcal{B}(G)^{0}\right)$ may be thought of as an arithmetic line bundle $\mathcal{L}$ on $L$ (i.e., a line bundle on $\operatorname{Spec}\left(\mathcal{O}_{L}\right)$, equipped with Hermitian metrics at the archimedean primes - cf. [Szp, pp. 13-14]). If $\mathcal{L}$ is an arithmetic line bundle on $L$, and $\mathcal{M}$ is an arithmetic line bundle on $M$ (where $M \subseteq \widetilde{F}$ is a finite extension of $F$ ), then one verifies immediately that a morphism $\mathcal{L} \rightarrow \mathcal{M}$ in $\mathcal{C}_{\widetilde{F} / F}$ may be thought of as consisting of the following data: (a) a morphism $\operatorname{Spec}(L) \rightarrow \operatorname{Spec}(M)$ over $\operatorname{Spec}(F)$; (b) an element $d \in \mathbb{N}_{\geq 1}$; (c) a nonzero morphism of arithmetic line bundles $\left.\mathcal{L}^{\otimes d} \rightarrow \mathcal{M}\right|_{L}$ on $L$. Also, we observe that for $A \in \operatorname{Ob}\left(\mathcal{C}_{\widetilde{F} / F}\right)$ that projects to $\operatorname{Spec}(L) \in \operatorname{Ob}\left(\mathcal{B}(G)^{0}\right)$, we have

$$
\mathcal{O}^{\times}(A)=\mathcal{O}^{\triangleright}(A)=\boldsymbol{\mu}(L)
$$

(cf., for instance, [Szp, p. 15]). Also, observe that we have a natural arithmetic degree homomorphism

$$
\operatorname{deg}_{L}^{\text {arith }}: \Phi(L)^{\mathrm{gp}} \rightarrow \mathbb{R}
$$

obtained as follows. If $v$ is archimedean, so we have a natural embedding of topological fields $\mathbb{R} \hookrightarrow F_{v}$, then the restriction of $\operatorname{deg}_{L}^{\text {arith }}$ to the factor ord $\left(F_{v}\right)$ maps the image of $\lambda \in \mathbb{R}_{>0}$ to $-\left[F_{v}: \mathbb{R}\right] \cdot \log (\lambda)$. If $v$ is non-archimedean, then the restriction of $\operatorname{deg}_{L}^{\text {arith }}$ to the factor $\operatorname{ord}\left(F_{v}\right)$ maps the image of an element $\lambda \in \mathcal{O}_{v}^{\triangleright}$ to the natural logarithm of the cardinality of the finite set $\mathcal{O}_{v} /(\lambda)$ (where $\mathcal{O}_{v}$ is the ring of integers of $F_{v}$ ). Thus, one verifies immediately that $\operatorname{deg}_{L}^{\text {arith }}$ vanishes on the image of $\mathbb{B}(L)$.

Remark 6.3.1. In light of Examples 6.1 and 6.3, many readers might expect that the next natural step is to attempt to apply the theory of Frobenioids to study arithmetic line bundles on higher-dimensional arithmetic varieties. This leads, however, to numerous complications which are beyond the scope of the present paper. Moreover, it is not even clear to the author at the time of writing that this constitutes a natural direction in which to further develop the theory of Frobenioids.

THEOREM 6.4. (Arithmetic Frobenioids) For $i=1,2$, let $F_{i}$ be a number field, $\widetilde{F}_{i} / F_{i} a$ (possibly infinite) Galois extension, $G_{i} \stackrel{\text { def }}{=} \operatorname{Gal}\left(\widetilde{F}_{i} / F_{i}\right), \mathcal{D}_{i} \stackrel{\text { def }}{=} \mathcal{B}\left(G_{i}\right)^{0}$, $\Phi_{i}$ the monoid on $\mathcal{D}_{i}$ given by the effective arithmetic divisors, $\mathbb{B}_{i}$ the group-like monoid on $\mathcal{D}_{i}$ given by the multiplicative group of the field extension of $F_{i}$ in question, $\mathbb{B}_{i} \rightarrow \Phi_{i}^{\mathrm{gp}}$ the natural map and

the associated model Frobenioid of Theorem 5.2(ii). Then we have the following. 
(i) Assume that the data labeled by the index ' 1 ' is equal to the data labeled by the index ' 2 ' (so in the remainder of the present assertion ( $i)$, we shall omit these indices). Then the Frobenioids $\mathcal{C}, \mathcal{C}^{\text {pf }}, \mathcal{C}^{\text {rlf }}, \mathcal{C}^{\text {un-tr }}$ and $\left(\mathcal{C}^{\text {pf }}\right)^{\text {un-tr }}$ are of isotropic and rationally standard type, but not of group-like type; $\mathcal{D}$ is Frobenius-slim and Div-slim (with respect to $\Phi, \Phi^{\mathrm{pf}}$ and $\left.\Phi^{\mathrm{rlf}}\right)$. Moreover, $\mathcal{D}$ is slim if and only if the subgroup of elements of $G$ that commute with some open subgroup of $G$ is trivial. Finally, if $A \in \mathrm{Ob}\left(\mathcal{C}^{\mathrm{rlf}}\right)$ is a Frobenius-trivial object that projects to the object of $\mathcal{D}$ determined by a finite extension $L \subseteq \widetilde{F}$ of $F$, then $\operatorname{deg}_{L}^{\text {arith }}$ determines an isomorphism of groups

$$
\delta_{A}: \operatorname{Pic}_{\Phi}(A) \stackrel{\sim}{\rightarrow} \mathbb{R}
$$

(cf. Theorem 5.1(i)).

(ii) Let

$$
\Psi^{\mathrm{rlf}}: \mathcal{C}_{1}^{\mathrm{rlf}} \stackrel{\sim}{\rightarrow} \mathcal{C}_{2}^{\mathrm{rlf}}
$$

be an equivalence of categories between the realifications (cf. Proposition 5.3) of $\mathcal{C}_{1}, \mathcal{C}_{2}$. Then there exists an element $\operatorname{deg}\left(\Psi^{\mathrm{rlf}}\right) \in \mathbb{R}_{>0}$ such that for all Frobeniustrivial $A_{1} \in \mathrm{Ob}\left(\mathcal{C}_{1}\right), A_{2} \in \mathrm{Ob}\left(\mathcal{C}_{2}\right)$ such that $A_{2}=\Psi^{\mathrm{rlf}}\left(A_{1}\right)$ (where we recall that $\Psi^{\mathrm{rlf}}$ preserves Frobenius-trivial objects - cf. (i) and Corollary 4.11(iv)), the composite of $\delta_{A_{2}}$ with the isomorphism $\operatorname{Pic}_{\Phi}\left(A_{1}\right) \stackrel{\sim}{\rightarrow} \operatorname{Pic}_{\Phi}\left(A_{2}\right)$ determined by $\Psi^{\text {rlf }}$ (cf. (i) above and Corollary 4.10 and $4.11($ iii $))$ is equal to $\operatorname{deg}\left(\Psi^{\mathrm{rlf}}\right) \cdot \delta_{A_{1}}$.

(iii) If the equivalence of categories $\Psi^{\mathrm{rlf}}$ of (ii) arises from an equivalence of categories

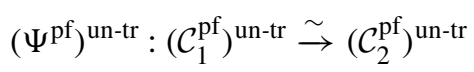

between the unit-trivialized perfections of $\mathcal{C}_{1}, \mathcal{C}_{2}$ (cf. (i) and Corollary 5.4), then $\operatorname{deg}\left(\Psi^{\mathrm{rlf}}\right) \in \mathbb{Q}_{>0}$. In particular, if $A_{1} \in \mathrm{Ob}\left(\left(\mathcal{C}_{1}^{\mathrm{pf}}\right)^{\mathrm{un}-\mathrm{tr}}\right)$ (whose projection to $\mathcal{D}_{1}$ we denote by $\operatorname{Spec}\left(L_{1}\right)$ ), $A_{2} \in \mathrm{Ob}\left(\left(\mathcal{C}_{2}^{\mathrm{pf}}\right)^{\mathrm{un}-\mathrm{tr}}\right)$ (whose projection to $\mathcal{D}_{2}$ we denote by $\left.\operatorname{Spec}\left(L_{2}\right)\right), A_{2}=\left(\Psi^{\mathrm{pf}}\right)^{\mathrm{un}-\mathrm{tr}}\left(A_{1}\right)$, then the bijection

$$
\mathbb{V}\left(L_{1}\right) \stackrel{\sim}{\rightarrow} \operatorname{Prime}\left(\Phi_{1}\left(L_{1}\right)\right) \stackrel{\sim}{\rightarrow} \operatorname{Prime}\left(\Phi_{2}\left(L_{2}\right)\right) \stackrel{\sim}{\rightarrow} \mathbb{V}\left(L_{2}\right)
$$

induced by $\left(\Psi^{\mathrm{pf}}\right)^{\mathrm{un}-\mathrm{tr}}$ (cf. (i) and Corollary 4.11(iii)) maps a valuation $v_{1} \in \mathbb{V}\left(L_{1}\right)$ lying over a valuation $v_{0}$ of $\mathbb{Q}$ to a valuation $v_{2} \in \mathbb{V}\left(L_{2}\right)$ lying over the valuation $v_{0}$ of $\mathbb{Q}$.

(iv) If the equivalence of categories $\Psi^{\mathrm{rlf}}$ of (ii) arises from an equivalence of categories

$$
\Psi: \mathcal{C}_{1} \stackrel{\sim}{\rightarrow} \mathcal{C}_{2}
$$

between $\mathcal{C}_{1}, \mathcal{C}_{2}$ (cf. (i), (iii) and Theorem 3.4(iii), (iv)), then $\operatorname{deg}\left(\Psi^{\mathrm{rlf}}\right)=1$. If, moreover, there exists a finite extension $L_{1} \subseteq \widetilde{F}_{1}$ of $F_{1}$ which is Galois over $\mathbb{Q}$, then the corresponding (i.e., via the equivalence $\mathcal{D}_{1} \stackrel{\sim}{\rightarrow} \mathcal{D}_{2}$ induced by $\Psi-c f$. (i) and Corollary 4.11(ii)) finite extension $L_{2} \subseteq \widetilde{F}_{2}$ of $F_{2}$ is isomorphic to $L_{1}$ in a fashion that is compatible with an isomorphism $F_{1} \cong F_{2}$.

Proof. First, we consider assertion (i). We have already seen in Example 6.3 that the Frobenioid $\mathcal{C}$ is of isotropic and birationally Frobenius-normalized type and that $\Phi$ is nonzero (so $\mathcal{C}$ is not of group-like type) and perf-factorial. As was observed in the proof of Theorem 6.2(iii), (iv), $\mathcal{D}$ is Frobenius-slim and of FSM-type, hence also of FSMFF-type. Moreover, since any automorphism of a number field that fixes all of the valuations of the 
number field is clearly equal to the identity automorphism, it follows immediately that $\Phi$ is non-dilating and that $\mathcal{D}$ is Div-slim (relative to $\Phi$, hence also relative to $\Phi^{\mathrm{pf}}, \Phi^{\mathrm{rlf}}$ ). Also, it is immediate from the definition of $\mathbb{B}$ that $\mathcal{C}$ is of (strictly) rational type and that every object of $\left(\mathcal{C}^{\text {un-tr }}\right)^{\text {birat }}$ is Frobenius-compact. Thus, we conclude that $\mathcal{C}$ (hence also $\mathcal{C}^{\text {pf }}, \mathcal{C}^{\text {rlf }}, \mathcal{C}^{\text {un-tr }}$, $\left(\mathcal{C}^{\mathrm{pf}}\right)^{\mathrm{un}-\mathrm{tr}}-\mathrm{cf}$. Proposition 5.5(iii)) is of rationally standard type. The proof of the criterion for $\mathcal{D}$ to be slim is entirely similar to the proof given for Theorem 6.2(iv). Finally, to show that the surjection

$$
\delta_{A}: \operatorname{Pic}_{\Phi}(A) \rightarrow \mathbb{R}
$$

is, in fact, an isomorphism, it suffices to verify that the image of $\Phi^{\text {birat }}(L) \otimes_{\mathbb{Z}} \mathbb{R}=\left(L^{\times}\right) \otimes_{\mathbb{Z}}$ $\mathbb{R}$ in $\left(\Phi_{\text {factor }}^{\mathrm{rlf}}\right)^{\mathrm{gp}}(L)$ is equal to the set of elements of $\left(\Phi_{\text {factor }}^{\mathrm{rlf}}\right)^{\mathrm{gp}}(L)$ with finite support whose image under $\operatorname{deg}_{L}^{\text {arith }}$ is 0 . But this is an immediate consequence of the well-known Dirichlet unit theorem of classical number theory (cf., e.g., [Lang2, p. 104]). This completes the proof of assertion (i).

Now assertion (ii) follows by observing that the isomorphism of groups

$$
\operatorname{Pic}_{\Phi}\left(A_{1}\right) \stackrel{\sim}{\rightarrow} \operatorname{Pic}_{\Phi}\left(A_{2}\right)
$$

determined by $\Psi^{\text {rlf }}$ (cf. assertion (i) and Corollaries 4.10 and 4.11(iii)) is compatible with the 'order structure' induced on both sides (via $\delta_{A_{1}}, \delta_{A_{2}}$ ) by the 'order structure' of $\mathbb{R}$. (Indeed, this compatibility follows from the fact that the isomorphism in question arises from an isomorphism of monoids $\Phi_{1}^{\mathrm{rlf}}\left(A_{1}\right) \stackrel{\sim}{\rightarrow} \Phi_{2}^{\mathrm{rlf}}\left(A_{2}\right)$.) This completes the proof of assertion (ii).

Next, we observe that assertion (iii) follows formally from assertion (ii) by applying Lemma 6.5(ii), below, in the following fashion. If $\operatorname{deg}\left(\Psi^{\mathrm{rlf}}\right) \notin \mathbb{Q}>0$, then one verifies immediately that there exist three non-archimedean valuations $w_{1}, w_{3}, w_{5} \in \mathbb{V}\left(L_{1}\right)$ lying over primes $p_{1}, p_{3}, p_{5} \in \mathfrak{P r i m e s}$, respectively, with the property that $w_{1} \mapsto w_{2} \in \mathbb{V}\left(L_{2}\right)$, $w_{3} \mapsto w_{4} \in \mathbb{V}\left(L_{2}\right), w_{5} \mapsto w_{6} \in \mathbb{V}\left(L_{2}\right)$, where $w_{2}, w_{4}, w_{6}$ lie over primes $p_{2}, p_{4}, p_{6} \in$ Primes, respectively, such that $p_{1}, p_{2}, p_{3}, p_{4}, p_{5}, p_{6}$ are distinct. But this implies that

$$
\log \left(p_{1}\right) / \log \left(p_{2}\right), \log \left(p_{3}\right) / \log \left(p_{4}\right), \log \left(p_{5}\right) / \log \left(p_{6}\right) \in\left(\operatorname{deg}\left(\Psi^{\mathrm{rlf}}\right)\right)^{-1} \cdot \mathbb{Q}_{>0}
$$

in contradiction to Lemma 6.5(ii). Thus, $\operatorname{deg}\left(\Psi^{\text {rlf }}\right) \in \mathbb{Q}>0$. The final portion of assertion (iii) concerning valuations of $\mathbb{Q}$ now follows from Lemma 6.5(i). This completes the proof of assertion (iii).

Finally, we consider assertion (iv). Suppose that $v_{1} \in \mathbb{V}\left(L_{1}\right)$ maps to $v_{2} \in \mathbb{V}\left(L_{2}\right)$ (cf. the notation of the statement of assertion (iii)). For $i=1$, 2, write

$$
\operatorname{deg}\left(L_{i}, v_{i}\right)
$$

for the number of valuations $\in \mathbb{V}\left(L_{i}\right)$, including $v_{i}$, that lie over the same valuation of $\mathbb{Q}$ as $v_{i}$. Then by Tchebotarev's density theorem (cf., e.g., [Lang2, Chapter VIII, Section 4, Theorem 10]), it follows that $\left[L_{i}: \mathbb{Q}\right]$ is equal to the maximum of the $\operatorname{deg}\left(L_{i}, v_{i}\right)$, as $v_{i}$ ranges over the elements of $\mathbb{V}\left(L_{i}\right)$. Moreover, if $v_{i}$ is non-archimedean and lies over a prime $p_{i} \in \mathbb{V}\left(L_{i}\right)$, then $p_{i}$ splits completely in $L_{i}$ if and only if $\operatorname{deg}\left(L_{i}, v_{i}\right)=\left[L_{i}: \mathbb{Q}\right]$. Thus, it follows that if $v_{1}, v_{2}$ lie over a prime $p \in \mathfrak{P r i m e s}$ (cf. assertion (iii)), then (again by assertion (iii)) $p$ splits completely in $L_{1}$ if and only if $p$ splits completely in $L_{2}$. If this is the case, then it follows that $\operatorname{deg}_{L_{i}}^{\text {arith }}$ maps a generator of the monoid $\Phi_{i}\left(L_{i}\right)_{v_{i}}\left(\cong \mathbb{Z}_{\geq 0}\right)$ to $\log (p)$. Thus, we conclude that $\operatorname{deg}\left(\Phi^{\mathrm{rlf}}\right)=1$, as desired. Note that this implies that $v_{1}$ is of 
degree 1 (i.e., $\operatorname{deg}_{L_{1}}^{\text {arith }}$ maps a generator of the monoid $\Phi_{1}\left(L_{1}\right)_{v_{1}}\left(\cong \mathbb{Z}_{\geq 0}\right)$ to $\left.\log (p)\right)$ if and only if $v_{2}$ is of degree 1 . Thus, if $L_{1}$ is Galois over $\mathbb{Q}$, then whenever $v_{2}$ is of degree 1 , it follows that $v_{1}$ is of degree 1 , hence that $p$ splits completely in $L_{1}$ (since $L_{1}$ is Galois over $\mathbb{Q}$ ). But this implies (again by Tchebotarev's density theorem - cf., e.g., [NSW, Theorem 12.2.5]) that $L_{1} \subseteq L_{2}$, hence that $L_{1}=L_{2}$ (since we have already seen that $\left[L_{1}: \mathbb{Q}\right]=\left[L_{2}: \mathbb{Q}\right]$ ). This completes the proof of assertion (iv).

LEMMA 6.5. (Transcendental properties of logarithms of prime numbers)

(i) The real numbers $\log (p) \in \mathbb{R}$, where $p$ ranges over the prime numbers, are linearly independent over $\mathbb{Q}$.

(ii) Let $p_{1}, p_{2}, \ldots, p_{6}$ be distinct prime numbers. Then there do not exist $\lambda_{1}, \lambda_{2} \in \mathbb{Q}_{>0}$ such that $\log \left(p_{1}\right) / \log \left(p_{2}\right)=\lambda_{1} \cdot \log \left(p_{3}\right) / \log \left(p_{4}\right)=\lambda_{2} \cdot \log \left(p_{5}\right) / \log \left(p_{6}\right)$.

Proof. Assertion (i) is a formal consequence of the fact that $\mathbb{Z}$ is a unique factorization domain. Assertion (ii) is a consequence of a theorem of Lang (cf. [Lang1] and [Baker, p. 119]). Indeed, since the $\log \left(p_{i}\right)$ are linearly independent over $\mathbb{Q}$ (by assertion (i)), it follows that each of the following two sets of numbers is also linearly independent over $\mathbb{Q}$ :

$$
\left\{\log \left(p_{2}\right), \log \left(p_{4}\right), \log \left(p_{6}\right)\right\}, \quad\left\{1, \log \left(p_{3}\right) / \log \left(p_{4}\right)\right\} .
$$

Moreover, all six products of one element from the first set and one element from the second set are of the form $\mu \cdot \log \left(p_{i}\right)$, where $\mu \in \mathbb{Q}_{>0}$. Thus, the exponential of each of these products is algebraic, in contradiction to Lang's theorem.

\section{Appendix. Slim exponentiation}

In this appendix, we discuss some elementary general nonsense concerning slim categories.

\section{Definition A.1.}

(i) A 2-category of 1-categories will be called 2-slim (cf. [Mzk7, Definition 1.2.4(iii)]) if every 1-morphism (i.e., functor) in the 2-category has no nontrivial automorphisms.

(ii) If $\mathcal{D}$ is a 2-category of 1-categories, then we shall write

for the associated 1-category whose objects are objects of $\mathcal{D}$ and whose morphisms are isomorphism classes of morphisms of $\mathcal{D}$ (cf. [Mzk7, Definition 1.2.4(iv)]). We shall also refer to $|\mathcal{D}|$ as the coarsification of $\mathcal{C}$.

Remark A.1.1. The name 'coarsification' is motivated by the theory of 'coarse moduli spaces' associated to (say) 'fine moduli stacks' (cf. [Mzk7, Remark 1.2.4.1]).

The following result may be regarded as a generalization of [Mzk7, Proposition 1.2.5(ii)], (a result concerning anabelioids), to the case of arbitrary slim categories.

Proposition A.2. (Slim exponentiation) Let $\mathcal{C}$ be a slim category ( $c f$. Section 0 ). Let $\mathcal{D}$ be the 2-category of 1-categories defined as follows: The objects of $\mathcal{D}$ are the categories $\mathcal{C}_{A}$ (cf. Section 0$)$, where $A \in \mathrm{Ob}(A)$. The 1-morphisms of $\mathcal{D}$ are the functors

$$
f_{!}: \mathcal{C}_{A} \rightarrow \mathcal{C}_{B}
$$


(cf. Section 0) induced by morphisms $f: A \rightarrow B$ of $\mathcal{C}$. The 2-morphisms of $\mathcal{D}$ are isomorphisms between these functors ( $f$. Section 0 ). Then $\mathcal{D}$ is 2-slim. Moreover, the functor

$$
\begin{gathered}
\mathfrak{E}: \mathcal{C} \rightarrow|\mathcal{D}|, \\
A \mapsto \mathcal{C}_{A}, \quad f \mapsto f !
\end{gathered}
$$

determines an equivalence of categories $\mathcal{C} \stackrel{\sim}{\rightarrow}|\mathcal{D}|$. We shall refer to the functor $\mathfrak{E}$ as the slim exponentiation functor.

Proof. The fact that $\mathcal{D}$ is 2-slim follows immediately from the assumption that $\mathcal{C}$ is slim. Now it is immediate from the definitions that $\mathfrak{E}$ is full and essentially surjective. To verify that $\mathfrak{E}$ is faithful, let us first observe that given any two morphisms $f, g: A \rightarrow B$ of $\mathcal{C}$, an isomorphism between the functors $f_{!}, g_{!}: \mathcal{C}_{A} \rightarrow \mathcal{C}_{B}$ determines an isomorphism between

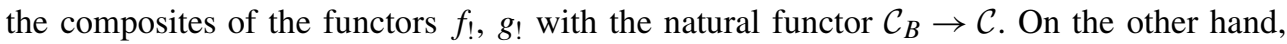
these two composite functors $\mathcal{C}_{A} \rightarrow \mathcal{C}$ both coincide with the natural functor $\mathcal{C}_{A} \rightarrow \mathcal{C}$ (i.e., that maps an object $C \rightarrow A$ of $\mathcal{C}_{A}$ to the object $C$ of $\mathcal{C}$ ). Thus, any isomorphism $f ! \stackrel{\sim}{\rightarrow} g$ ! determines an automorphism of the natural functor $\mathcal{C}_{A} \rightarrow \mathcal{C}$, which (by the slimness of $\mathcal{C}$ !) is the identity automorphism. But this implies (by applying the isomorphism $f_{!} \stackrel{\sim}{\rightarrow} g_{\text {! }}$ to the object $A \stackrel{\mathrm{id}_{A}}{\longrightarrow} A$ of $\mathcal{C}_{A}$ ) that $f=g$, as desired.

\section{Chart of types of morphisms in a Frobenioid}

\begin{tabular}{lccc}
\hline Type of morphism & Projection to base & Zero divisor & Frobenius degree \\
\hline Linear & $?$ & $?$ & 1 \\
Isometry & $?$ & 0 & $?$ \\
Base-isomorphism & Isomorphism & $?$ & $?$ \\
Base-FSM-morphism & FSM-morphism & $?$ & $?$ \\
Pull-back morphism & $?$ & 0 & 1 \\
Pre-step & Isomorphism & $?$ & 1 \\
Step & Isomorphism & $\neq 0$ & 1 \\
Primary pre-step & Isomorphism & Primary & 1 \\
Isometric pre-step & Isomorphism & 0 & 1 \\
LB-invertible & $?$ & 0 & $?$ \\
Morphism of Frobenius type & Isomorphism & 0 & $?$ \\
Prime-Frobenius morphism & Isomorphism & 0 & Prime \\
\hline
\end{tabular}

Acknowledgements. I would like to thank Akio Tamagawa, Makoto Matsumoto, Kazuhiro Fujiwara, and Seidai Yasuda for various useful comments. Also, I would like to thank Yuichiro Taguchi for inviting me to speak at Kyushu University during the Summer of 2003 on a preliminary version of the theory discussed in this paper.

\section{REFERENCES}

[Baker] A. Baker. Transcendental Number Theory. Cambridge University Press, 1975.

[Lang1] S. Lang. Algebraic values of meromorphic functions II. Topology 5 (1966), 363-370.

[Lang2] S. Lang. Algebraic Number Theory. Addison-Wesley, 1970.

[Mzk1] S. Mochizuki. A theory of ordinary p-adic curves. Publ. RIMS 32 (1996), 957-1151. 
[Mzk2] S. Mochizuki. Foundations of $p$-adic Teichmüller Theory (AMS/IP Studies in Advanced Mathematics, 11). American Mathematical Society/International Press, 1999.

[Mzk3] S. Mochizuki. A survey of the Hodge-Arakelov theory of elliptic curves I. Arithmetic Fundamental Groups and Noncommutative Algebra (Proceedings of Symposia in Pure Mathematics, 70). American Mathematical Society, 2002, pp. 533-569.

[Mzk4] S. Mochizuki. A survey of the Hodge-Arakelov theory of elliptic curves II. Algebraic Geometry 2000, Azumino (Adv. Stud. Pure Math., 36). Math. Soc. Japan, 2002, pp. 81-114.

[Mzk5] S. Mochizuki. The absolute anabelian geometry of hyperbolic curves. Galois Theory and Modular Forms. Kluwer Academic Publishers, 2003, pp. 77-122.

[Mzk6] S. Mochizuki. The absolute anabelian geometry of canonical curves. Kazuya Kato's fiftieth birthday, Doc. Math. (2003), Extra Vol., 609-640.

[Mzk7] S. Mochizuki. The geometry of anabelioids. Publ. Res. Inst. Math. Sci. 40 (2004), 819-881.

[Mzk8] S. Mochizuki. Categorical representation of locally noetherian log schemes. Adv. Math. 188 (2004), 222-246.

[Mzk9] S. Mochizuki. Categories of log schemes with Archimedean structures. J. Math. Kyoto Univ. 44 (2004), 891-909.

[Mzk10] S. Mochizuki. Galois sections in absolute anabelian geometry. Nagoya Math. J. 179 (2005), 17-45.

[Mzk11] S. Mochizuki. Semi-graphs of Anabelioids. Publ. Res. Inst. Math. Sci. 42 (2006), 221-322.

[Mzk12] S. Mochizuki. A combinatorial version of the Grothendieck conjecture. Tohoku Math J. 59 (2007), 455-479.

[Mzk13] S. Mochizuki. Conformal and quasiconformal categorical representation of hyperbolic riemann surfaces. Hiroshima Math. J. 36 (2006), 405-441.

[Mzk14] S. Mochizuki. Absolute anabelian cuspidalizations of proper hyperbolic curves. J. Math. Kyoto Univ. 47 (2007), 451-539.

[Mzk15] S. Mochizuki. The geometry of Frobenioids II: poly-Frobenioids. RIMS Preprint, 1530 (February 2006).

[Mzk16] S. Mochizuki. The étale theta function and its Frobenioid-theoretic manifestations, RIMS Preprint 1569 (September 2006); updated version available at: http://www.kurims.kyoto-u.ac.jp/ motizuki/papers-english.html.

[NSW] J. Neukirch. A. Schmidt and K. Wingberg. Cohomology of Number Fields (Grundlehren der Mathematischen Wissenschaften, 323). Springer-Verlag, 2000.

[Szp] L. Szpiro. Degrés, intersections, hauteurs. Astérisque 127 (1985), 11-28.

\section{Index}

1-commutative, Section 0

2-slim, Definition A.1(i)

abstract equivalence, Section 0

almost totally epimorphic, Section 0

anchor, Section 0

$A$-pair, proof of Theorem 5.1(i)

arithmetic degree, Example 6.3

arithmetic divisor, Example 6.3

arithmetic line bundle, Example 6.3

Aut-ample, Definition 1.2(iv), (v)

Aut-saturated, Section 0

$\mathrm{Aut}^{\mathrm{sub}}$-ample, Definition 1.2(iv), (v)

Aut ${ }^{\text {sub }}$-saturated, Section 0

Aut-type, Section 0

base category, Definition 1.1(iii), (iv)

base-equivalent, Definition 1.2(ii)
base-Frobenius pair (of a Frobenioid), Definition 2.7(iii)

base-Frobenius pair (of a Frobenius-trivial object), Proposition 5.6

base-FSM-morphism, Definition 1.2(ii)

base-identity, Definition 1.2(ii)

base-isomorphism (base-isomorphic),

Definition 1.2(ii)

base-section, Definition 2.7(i)

base-trivial, Definition 1.2(iv), (v)

birationalization (of a Frobenioid),

Proposition 4.4

birationally Frobenius-normalized,

Definition 4.5(i)

bounded, Section 0

categorical fiber product, Section 0

categorical quotient, Section 0 
centralizer, Section 0

characteristic, Section 0

characteristically injective, Section 0

characteristic splitting, Definition 2.3

characteristic type, Section 0

co-angular, Definition 1.2(iii)

coarsification, Definition A.1(ii)

connected category, Section 0

co-objective, Section 0

co-primary, Proposition 4.1(iii)

co-prime type, Definition 2.8(iii)

Div-equivalent, Definition 1.2(ii)

Div-Frobenius-trivial, Definition 1.2(iv), (v)

Div-identity, Definition 1.2(ii)

divisorial, Definition 1.1(i)

divisor monoid, Definition 1.1(iv);

Theorem 5.2(ii)

Div-slim, Definition 4.5(iv)

effective arithmetic divisor, Example 6.3

elementary Frobenioid, Definition 1.1(iii)

End-ample, Definition 1.2(iv), (v)

End-equivalence, Section 0

factorization homomorphism,

Definition 2.4(i)(c)

factorization of morphisms of a Frobenioid,

Definition 1.3(iv)(a)

factorization of pre-steps of a Frobenioid,

Definition 1.3(v)(b), (c)

$\mathcal{F}$-distinguished, Definition 2.7(ii)

fiberwise surjective, Section 0

finitely (respectively, countably) connected

type, Section 0

$\mathcal{F P}$-path, proof of Theorem 5.2(iv)

Frobenioid, Definition 1.3

Frobenius-ample, Definition 1.2(iv), (v)

Frobenius-compact, Definition 1.2(iv), (v)

Frobenius degree, Definition 1.1(iii), (iv)

Frobenius functor (on an elementary

Frobenioid), Definition 2.4(iii)

Frobenius-isotropic, Definition 1.2(iv), (v)

Frobenius-normalized, Definition 1.2(iv), (v)

Frobenius-section, Definition 2.7(ii)
Frobenius-slim, Definition 3.1(i)

Frobenius-trivial, Definition 1.2(iv), (v)

FSMFF-type (category of), Section 0

FSMI-morphism, Section 0

FSM-morphism, Section 0

FSM-type (category of), Section 0

groupification, Section 0

group-like (monoid), Definition 1.1(i)

group-like (object of a pre-Frobenioid),

Definition 1.2(iv), (v)

immobile, Section 0

integral, Section 0

irreducible (element of a monoid), Section 0

irreducible (morphism of a category),

Section 0

isometric morphism (isometry),

Definition 1.2(i)

iso-subanchor, Section 0

isotropic, Definition 1.2(iv), (v)

isotropic hull, Definition 1.2(iv)

isotropification functor, Proposition 1.9(v)

$\widetilde{K}$-QQ-Cartier, Example 6.1

LB-invertible, Definition 1.2(iii)

left-hand isomorphism, Theorem 4.2(iii)

linear morphism, Definition 1.2(i)

metrically equivalent, Definition 1.2(i)

metrically trivial, Definition 1.2(iv), (v)

mid-adjoint, Section 0

minimal-adjoint, Section 0

minimal-coadjoint, Section 0

mobile, Section 0

model Frobenioid, Theorem 5.2(ii)

model type, Definition 4.5(i)

monoid, Section 0

monoid (on a category), Definition 1.1(ii)

monoid type, Section 0

mono-minimal, Section 0

monoprime, Section 0

morphism of Frobenius type,

Definition 1.2(iii)

naive Frobenius functor, Proposition 2.1(i) 
natural projection functor, Definition 1.1(iii), rational function monoid,

(iv)

non-dilating (endomorphism),

Definition 1.1(i)

non-dilating (monoid on a category),

Definition 1.1(ii)

number field, Section 0

one-morphism category, Section 0

one-object category, Section 0

opposite category, Section 0

$\left(p_{1}, p_{2}\right)$-admissible, proof of Theorem 3.4

$\mathcal{P}$-distinguished, Definition 2.7(i)

perfection (of a Frobenioid),

Definition 3.1(iii)

perfection (of a monoid), Section 0

perfect (monoid), Section 0

perfect (object of a pre-Frobenioid),

Definition 1.2(iv)

perf-factorial, Definition 2.4(i)

pre-divisorial, Definition 1.1(i)

pre-Frobenioid, Definition 1.1(iv)

pre-Frobenioid structure, Definition 1.1(iv)

pre-model type, Definition 2.7(iii)

pre-step, Definition 1.2(iii)

primary (element of a monoid), Section 0

primary (pre-step), Definition 1.2(iii)

prime, Section 0

prime-Frobenius morphism,

Definition 1.2(iii)

pro- $l$ portion, Definition 2.8(ii)

pseudo-terminal, Section 0

pull-back morphism, Definition 1.2(ii)

quasi-base-Frobenius pair (of a Frobenioid),

Definition 2.7(iii)

quasi-base-Frobenius pair (of a

Frobenius-trivial object),

Proposition 5.6

quasi-connected, Section 0

quasi-Frobenius-section, Definition 2.7(ii)

quasi-Frobenius-trivial, Definition 1.2(iv)(v)

quasi-isotropic type, Definition 3.1(i)

raising to the $\zeta$-th power, Definition 2.8(iii)
Proposition 4.4(ii); Theorem 5.2(ii)

rationally standard type, Definition 4.5(iii)

rational object, Definition 4.5(ii)

rational type, Definition 4.5(ii)

realification (of a Frobenioid),

Proposition 5.3

realification (of a perf-factorial monoid),

Definition 2.4(i)

residually finite group, Remark 3.1.2

right-hand isomorphism, Theorem 4.2(iii)

rigid, Section 0

saturated, Section 0

sharp, Section 0

skeletal subcategory, Section 0

skeleton, Section 0

slim (category), Section 0

slim exponentiation functor, Proposition A.2

slim (profinite group), Section 0

standard Frobenioid, Definition 1.1(iii)

standard type, Definition 3.1(i)

step, Definition 1.2(iii)

strictly rational object, Definition 4.5(ii)

strictly rational type, Definition 4.5(ii)

subanchor, Section 0

sub-automorphism, Section 0

subordinate, Section 0

sub-quasi-Frobenius-trivial,

Definition 1.2(iv), (v)

supporting monoid type, Definition 2.4(ii)

support (of an element of a perf-factorial

monoid), Definition 2.4(i)(d)

supremum, Section 0

terminal, Section 0

totally epimorphic, Section 0

twin-primary, proof of Theorem 4.9

unit-equivalence, Definition 3.1(iv)

unit-linear Frobenius functor,

Proposition 2.5(iii)

unit-profinite type, Definition 2.8(i)

unit-trivial, Definition 1.2(iv), (v) 
unit-trivialization (of a Frobenioid), Definition 3.1(iv)

unit-wise Frobenius functor, Corollary 2.6;

Proposition 2.9 universally Div-Frobenius-trivial,

Definition 1.2(iv), (v)

zero divisor, Definition 1.1(iii), (iv)

\author{
Shinichi Mochizuki \\ Research Institute for Mathematical Sciences \\ Kyoto University \\ Kyoto 606-8502 \\ Japan \\ (E-mail:motizuki@kurims.kyoto-u.ac.jp)
}

